

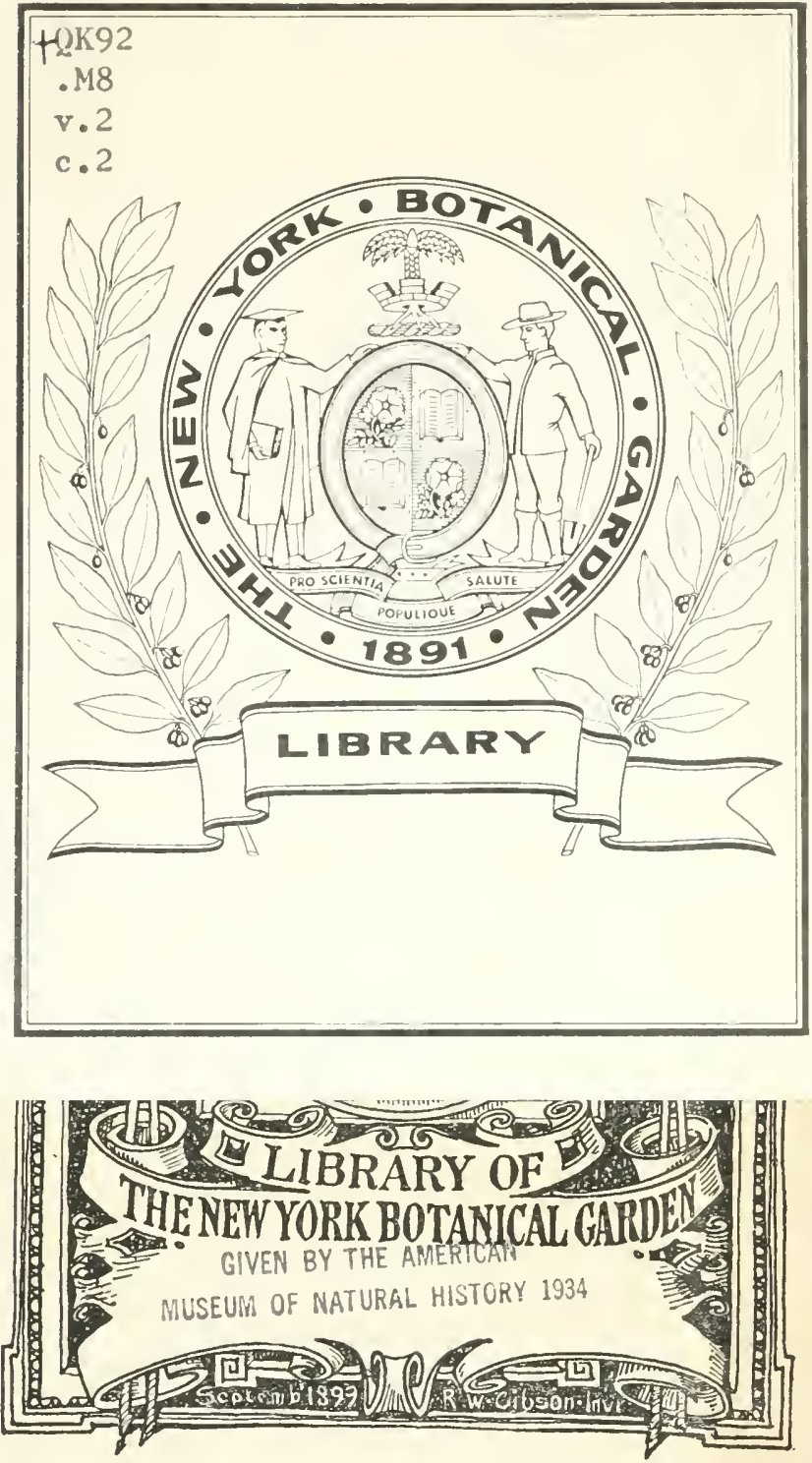








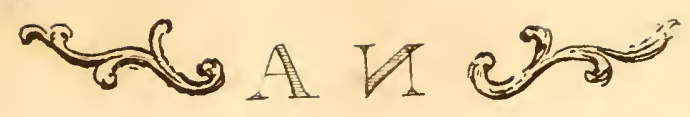

\section{ILUUSTRATION of the}

\section{TERMINI}

$B O T A N \mathbb{I} \mathbb{L}$

O F BOAANCAL QARDEN

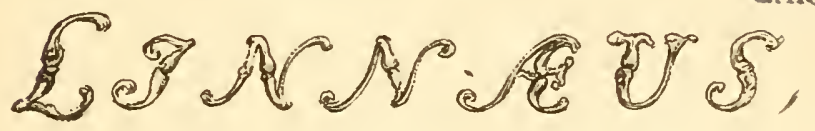

by

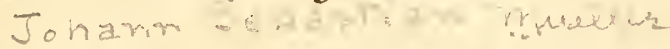

\section{IOHN MILIER.}

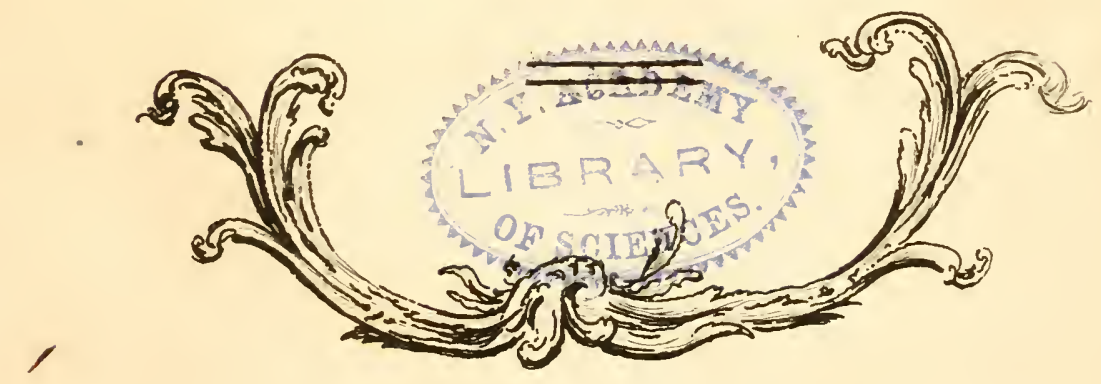

Publifhed as the Aet divects.

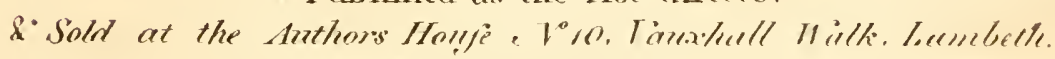
MDCCI.XXXIX.

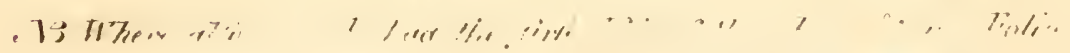

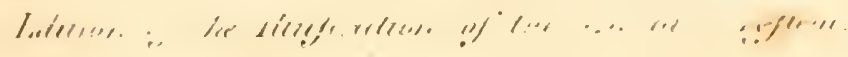


.$M \varepsilon$

C. 2 


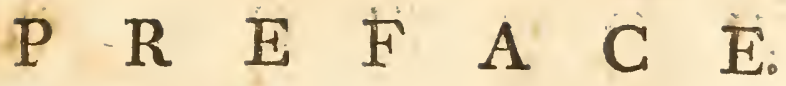

WHEN I, in the Year 1779, publifhed the Sexual Syfem of Linnæus, in Octavo, I then promifed to give in, the following Year, the TERMINI BOT ANICI, viz. RATIFICATION, RAMIFICATION, FOLIATION, GEMMATION, zc. \&zc. of Linnæus, in Figures, in like manner as has been given in the Sexual Syfem; but being by a particular Engagement prevented from the fulfilment of that Promife 'till the prefent Time:-I flatter myfelf, that a faithful delineation of each Term, from a Subject of Nature, that has, and expreffes, the character of the Terms, will convey a more eafy and accurate conception to the Mind of the Student and Lover of Botanic Science, than a verbal Defcription only.

It was found neceffary to retain the Latin Terms of Linnæus, but to give them an Englifh termination, with an Explanation of each Term, which it is hoped, will make it cafy and familiar to the Englifh Reader. 


\section{$P \quad R \quad E \quad F \quad A \quad C E$.}

I doubt not but fome Critic will, and may, find fault; in anfwer thereto I recommend the old Adage, (nofce de ip(e) and it is eafier to find Faults than to mend them: $I$ hope the candid and unprejudiced Reader will look upon the few Errors with an indulgent Eye. 


\section{( I)}

R. A D I X.

The Root, is the Origin of Nutrition of P L A N T S.

\section{Their D U R A T I O N:}

A $\mathrm{N} \underset{\text { Annual. }}{\mathrm{N}} \mathrm{U}$ A. $\{$ Abiding one Year only.

B.I E N N I S.
Biennual.

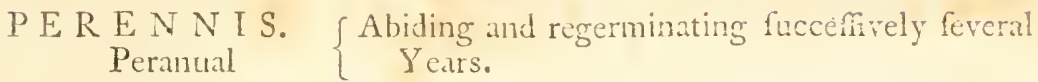

\section{Their FIGURE.}

F I B R O S A. Fig. I. Fibrous.
$\{$ Anthoxanthum.

Vernal Grafs. $\left\{\begin{array}{l}\text { confining of Fibres or } \\ \text { Filaments only }\end{array}\right.$

$\mathrm{R} A \mathrm{MOS}$, Fig. 2. Ramofes. $\left\{\begin{array}{l}\text { as the Trees and } \\ \text { branching Fibres. }\end{array}\right.$ $\left.\begin{array}{c}\text { F U S I F O R NIIS. } \\ \text { Fig 3. }\end{array}\right\}$ Raphanus. Fuffiform.

P R $\mathbb{E} M O R S A$.

Fig. 4 . Præmors.

RE P E N S. Fig. 5.

Repent. $\int$ as if bitten ofit at their ends.

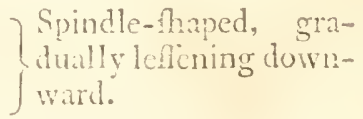

ubs fubdivide into

Spindle-miped, gra-
ilually leflening dowra-
ward. 


\section{(2) \\ R A M I. X.}

\section{Their FIGURE.}

$\underset{\substack{\text { Fig. I. } \\ \text { Globus. }}}{\text { GL O O'S }} \quad\left\{\begin{array}{l}\text { Ranunculus-bulbofus. } \\ \text { Butter-cup. }\end{array}\right\}$ Rounl.

TU BER OSA. TPœonia-sficinalis. T Carnos or Flefhy conFig. 2 . Tuberos.

Liony.

nected together by

$\int$ flender Fibres.

FASICULARIS.

Fig. 3 .

Faficuled.

$\left\{\right.$ Rinunculus-afracicus. $\left\{\begin{array}{l}\text { Flefhy and feffile at the } \\ \text { bafe of the Footitalk of } \\ \text { the Leaves. }\end{array}\right.$

PA I M T A. Fig. 4. Palmated.

$\left\{\begin{array}{l}\text { Orchis-latifolia. } \\ \text { broad-leaved Orchis. }\end{array}\right\}$ fhaped like a Hand.

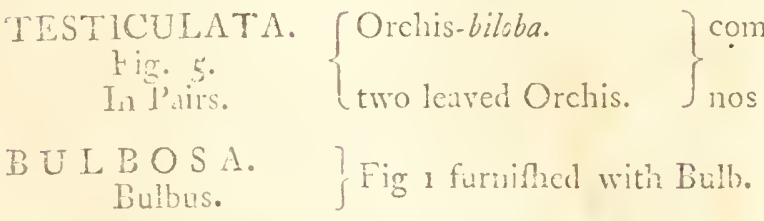

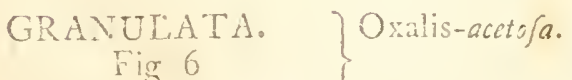

Gignuluted. $\int W^{2}$ cod-forrel.

Thaving Grain like Particles, difperfed on the fibres.

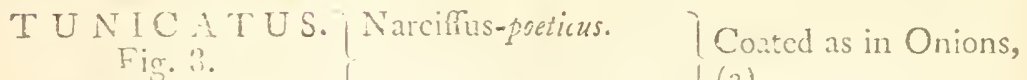
Tunicated. $\int$ Dafodil.

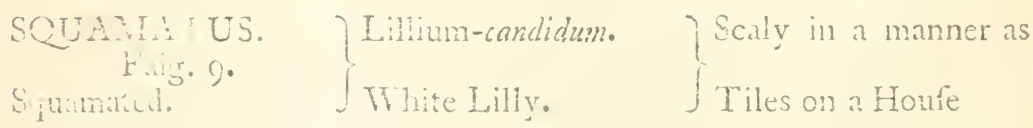




\section{$(3)$}

\section{T R U N G U S.}

The TRUnK or Sten, is the Origin which fupports the Branches, Leaves and Fructification.

\section{Their K I N D S.}

C A U L I S. Fig. 1. Stem.

C U L M U S. Fig. 2. A Culm.

$S$ C A P U S. Fig. 3. Stalk.

S T I P E S. Fig. 4. A Stipe.
$\{$ which elevates the Fructification and Leaves. the proper Stem of
Grafies, Onts, Wheat,
Exc. ) elevating the FructifiCation only, and no
Leaves. Daffodil.

$$
\left\{\begin{array} { l } 
{ \text { Polypodium-aculeatum } } \\
{ \text { prickly Polypodium. } }
\end{array} \left\{\begin{array}{l}
\text { is a Trunk or Stem } \\
\text { that expands itfeif into } \\
\text { a Leaf, and is alio ap- } \\
\text { plied to the Funci or } \\
\text { Muthrooms, Fig. 5. }
\end{array}\right.\right.
$$




\section{[ 4 ] $]$ \\ $T R \quad \mathrm{R} \quad \mathrm{C} \quad \mathrm{U} S$.}

\section{Their D URATION.}

IIER S. ACEUS. A Alifma-Plantago. Fig. I.

Herbaccous.

Wrater Plantain.

Herb-like, perifhes eYvery Year, an an ual $\int$ Stem, not woody.

SUFERUTICOSUS / Rubus-ideus.

Fig. 2.

Suffrutious.

Rafpherry.

half fhruoby, the Root permanent, thebranches fometimes wither-
ing.

ARBOREUS.

Arboreous.

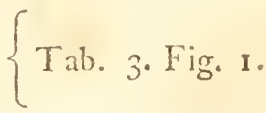

Tree like, a fingle

woody ftem, arifing

from the fame hoot.

FRUTICOSUS.

Fig 3 :

Fruticole.

$\left\{\begin{array}{l}\text { Ribra-rubia. } \\ \text { Currans. }\end{array}\right.$

) both ftem and branch

$\int$ es woody and abiling.

SOLIDUS. Fig 4 . soliu.

$\left\{\begin{array}{l}\text { without internal Pores. } \\ \text { Fig. } 1 \cdot 3 \cdot\end{array}\right.$

(b) vide Tab. I I.

NIS.

Fig. 5 .

I'ithy.

$\left\{\begin{array}{l}\text { Alce-rojaa. } \\ \text { Holl hock. }\end{array}\right.$

\}aving internally a

$\int$ frongy Subitance. (a)

FISTULOSIS.

Fic. 6 。

Fifiulous.

$\{$ Fig. (c.) 


\section{$\left[\begin{array}{ll}5 & \end{array}\right]$ \\ $\begin{array}{lllllll}\mathrm{T} & \mathrm{R} & \mathrm{U} & \mathrm{N} & \mathrm{C} & \mathrm{U} & \mathrm{S} \text {. }\end{array}$}

\section{Their D I REC TION.}

ER E E C T U S. $\left\{\begin{array}{l}\text { Dipfacus-laciniains. } \\ \text { Erect. }\end{array}\right\} \begin{aligned} & \text { rifing nearly to a per- } \\ & \text { pendicular Direction. }\end{aligned}$

$\left\{\begin{array}{c}\text { S T R I C T U S: } \\ \text { Straight. }\end{array}\right.$

R I G I D U S. $\quad\{$ hard, not eafily bent.

L A X U S. Fig. 2. Lax.

$\left\{\begin{array}{c}\text { Zanichellia-paluftris. } \\ \text { ripe-headed Ṕond- } \\ \text { weed. }\end{array}\right\}$ loofe, eaflly bent.

O B L I CUUS. Fig. 3. Oblique.

$\left\{\begin{array}{l}\text { Solidago-Aurea. } \\ \text { Golden Rod. }\end{array}\right.$

ADSCENDENS.
Fig. 4.
Afcending. $\quad\left\{\begin{array}{l}\text { Salvia oficinalis. } \\ \text { Garden Sage. }\end{array}\right\} \begin{aligned} & \text { rifing upwards with a } \\ & \text { Curve like an Arch. }\end{aligned}$

ADSCENDENS.
Fig. 4.
Afcending. $\quad\left\{\begin{array}{l}\text { Salvia offinalis. } \\ \text { Garden Sage. }\end{array}\right\} \begin{aligned} & \text { rifing upwards with a } \\ & \text { Curve like an Arch. }\end{aligned}$

\}awry, neither prepen-

$\int$ dicular nor horizontal.

DECLINATUS.
Fig. 5.
Declining. $\quad\left\{\begin{array}{l}\text { Andrachne-delepbioides } \\ \text { Baftard-orpin. }\end{array}\right\} \begin{aligned} & \text { bending or declining } \\ & \text { downwards. }\end{aligned}$

INCURVATUS. Fig. 6. Incurved.

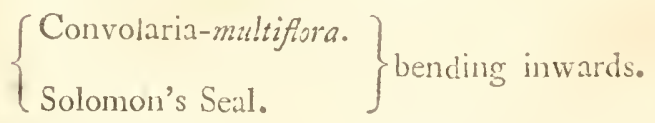

N U T A N S. Fig. $\%$ Nutant. $\left\{\begin{array}{l}\text { Salvia-nutans. } \\ \text { Nodding Sage. }\end{array}\right\} \begin{aligned} & \text { when the Top or Head } \\ & \text { bending downward. }\end{aligned}$ 


\section{$\left[\begin{array}{ll}6 & ]\end{array}\right.$

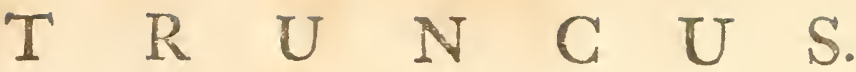

\section{The DIRECTION.}

DIFF USUS.

Fig. I.

Diffufe.

$\left\{\begin{array}{l}\text { Fumaria-lutea. } \\ \text { Yellow Fumatory. }\end{array}\right\} \begin{aligned} & \text { With fpreading } \\ & \text { Branches. }\end{aligned}$

PROCUMBENS.
Fig. 2.

Procumbent. Cucumber.

$\int$ and Fig. 3. STOLONIFESUS.
Fig. 3.
Rooting. $\quad\left\{\begin{array}{l}\text { Fragaria vefca. } \\ \text { Strawberry. }\end{array}\right.$

producing Shoots or Rumners from the

SARMENTOSUS.
Fig. 4.

Twiged.

Tine. Roots.

RE P E N S. \} long Shoots or Twigs. Repent. $\quad$ and here and there producing Roots.

RADICANS. Fig. 5 . Radicant. $\left\{\begin{array}{l}\text { Bignonia radicans. } \\ \text { Trumpet Flower. }\end{array}\right.$ Striking Rootslateraly and tixing to other Bodies. 


\section{( 7 ) \\ T $R \quad U \quad N \quad C \quad U S$. THE DIRECTION.}

GENICULATUS.

Fig. I.
Geniculated.

F L E X U O S U S. $\{$ Sinilax-aspera. Fig. 2. Flexuoufe.

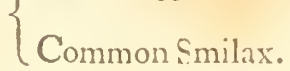

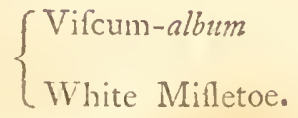

\}$_{\text {knots }}^{\text {divided by joints or }}$
SCANDENS
Fig. 3 . Climbing. Clinging.
V O L U B I L I S. Convolvulus-major. T climbing round fome

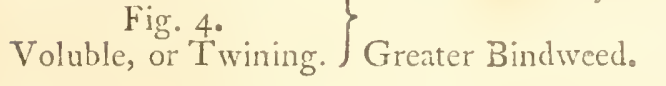

waved backwards and
forwards from bud to
bud.

DEXTROSUM. Fig. 3. Twining from the Right to the Left,

SINISTRORSUM, Fig. 4 Twining from the Left to the Right. 


\section{(8) \\ $\begin{array}{lllllll}\mathrm{T} & \mathrm{R} & \mathrm{U} & \mathrm{N} & \mathrm{C} & \mathrm{U} & \mathrm{S} .\end{array}$}

\section{The FIG U R E.}

TE R E S.
Fig. 1.
Round. $\quad\left\{\begin{array}{l}\text { Leontodon-teraxacum. } \\ \text { Dandelion. }\end{array}\right\} \begin{aligned} & \text { cylinder fhaped, withs } \\ & \text { out Angles. }\end{aligned}$ S E M I T E R E S.
Half round. $\left\{\begin{array}{l}\text { Amarillis-Belladona. } \\ \text { Belladona-Lilly. }\end{array}\right\}$ femi-cylindrical.

COMPRESSUS.
Fig. 3. $\left\{\begin{array}{l}\text { Stratiodes, aloides. } \\ \text { Water Aloe, or Wa- }\end{array}\right\}$ flattened, with two op-s Fig. 3.
Compreffed. $\quad\left\{\begin{array}{l}\text { Water Aloe, or } \mathrm{W}^{\top} \mathrm{a} \\ \text { ter-Soldier. }\end{array}\right\}_{\text {pofite Sides fat. }}$

A N C E P S.
Fig. 4.
Two-erlged. $\quad\left\{\begin{array}{l}\text { Hipericum-perforatum } \\ \text { St. John's-wort. }\end{array}\right\} \begin{aligned} & \text { flattened with two } \\ & \text { fharp Sides. }\end{aligned}$

ANGULATUS. $\quad$ Monarda-fisulofa having Angles,

$\begin{aligned} & \text { Fig. } 5 \\ & \text { Angled. }\end{aligned} \quad\left\{\begin{array}{l}\text { vide. Tab. 9. Fig. I, } \\ \text { Ofwego-Tea. }\end{array}, 3,4,5,6,7\right.$.

ACUTANGUI,US.
Acutely Angled.

OBTUSANGULUS $\{$ Fig. 5 .
Obtufely angled. 


\section{(9) \\ $\begin{array}{lllllll}T & R & U & N & G & U & S\end{array}$}

\section{The FIG U R F.}

$\left.\begin{array}{c}\text { TR I QUE T E R. }\left\{\begin{array}{l}\text { Scirpus-mucionatus. } \\ \text { Fig. I. } \\ \text { Three Sided. }\end{array}\right\} \text { Pointed Club-rufh. }\end{array}\right\}$ three Angles,

QUADRANGÜ-

L A R IS.

Fig. 2.

Four angeld

$\left\{\begin{array}{c}\text { Monarda-ffiulofa. } \\ \text { Ofwego Tea. }\end{array}\right\}$

QUINQUEANGU-

L A R IS.

Fig. 3 .

Five angled.

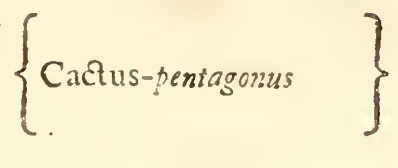

HEXANGULARIS.

Fig. 4 .

Six angled.

HEPTANGULA-

RIS.

Fig. 5 .

Seven angled.

Castusoheragonus:

\}

OCTANGULARIS

Fig. 6.

Eight angled.

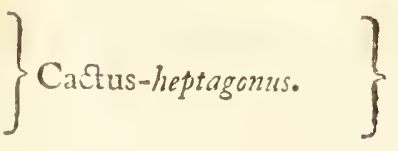

POLYANGULARIS.

Many 4 ingles.
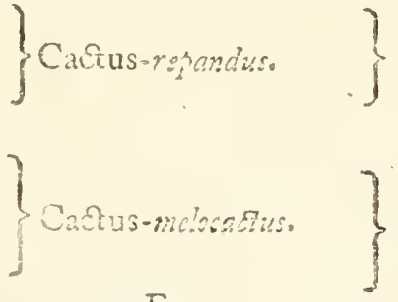


\section{(10) \\ $\begin{array}{lllllll}T & R & U & N & G & U & S\end{array}$}

The VESTITU.

NUDUS, or Aphyllus.

Fig. I.

Without Leaves.

$\{$ Caffytha-baccifera. $\} \begin{aligned} & \text { having no Leaves or } \\ & \text { other Covering. }\end{aligned}$

FO L I A T U S.
Fig. 2.
With Leaves. $\quad\left\{\begin{array}{c}\text { Oenethera-biennis. } \\ \text { Tree Primrofe. }\end{array}\right\}$ furnifhed with Leaves.

VAG IN A T US. \{ Rheum-palmatum. T furrounded with a Fig. 3. $\quad$ Spathaor Sheath at the Vaginate. Rheubarb. Bafe of the Leaves.

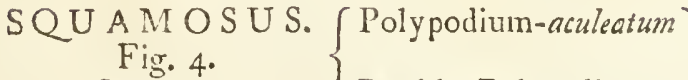
Squamus. $\left\{\begin{array}{l}\text { Prizkly-Polypodium. }\end{array}\right\}$ covered with Scales. $\left.\begin{array}{l}\text { I M B R I C A T US. }\{\text { Sempervivum-tectorum } \\ \text { Fig. 5. }\end{array}\right\} \begin{aligned} & \text { covered with Leaves } \\ & \text { placed like Tiles, or }\end{aligned}$ Imbricated. G Great Houfelcek. \}the Scales of Fifhes. 


\section{[ II ] \\ $\begin{array}{lllllll}\mathrm{T} & \mathrm{R} & \mathrm{U} & \mathrm{N} & \mathrm{C} & \mathrm{U} & \mathrm{S} .\end{array}$}

The SUPERFICIES.

SUB E R OSUS.
Fig. I.
Subcrous. $\left\{\begin{array}{l}\text { Quercus-juber. } \\ \text { Cork-Tree. }\end{array}\right.$

R I M O S U S.

Fig. 2.

Rimous.

$\left\{\begin{array}{l}\text { Quercus-robur. } \\ \text { Oak-Tree. }\end{array}\right.$
T U N I C A T U S. $\{$ Bedula-alba.
Fig. 3. Tunicated.

Birch-Tree.

\}$_{\text {tic like Cork. }}^{\text {the Bark fort but elar- }}$

\}$_{\text {and Fiffures. }}^{\text {the Bark full of } \text { rack }_{\mathrm{s}}}$

the Bark coated with

$\int$ Skins and Membranes.

I $E$ V I S. $\{$ Polygonum-Fagopyrum $\}$ free from ProtuberanFig. 4. $\quad\{$ Buck-Wheat. $\}$ ces and Inequalities.

Fig. 5.
Striated. $\quad\left\{\begin{array}{l}\text { Arum-maculatum. } \\ \text { Wake-Robin. }\end{array}\right\} \begin{aligned} & \text { marked with fimall } \\ & \text { Lines. }\end{aligned}$

S U L C A T U S.
Fig. 6.
Sulcated. $\quad\left\{\begin{array}{l}\text { Ranunculus-bulbofus. } \\ \text { Crowfoot or Butter- } \\ \text { Cup. }\end{array}\right\}_{\text {hollow Lines. }}^{\text {furrowed with deep }}$

G L A B E R. Fig. 7 . Smooth.

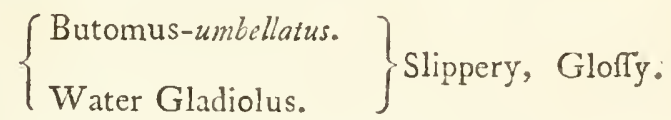

$S \mathrm{C} A \mathrm{~B} \mathrm{E} \mathrm{R}$. Fig. 8. Scabrous.

$$
\left\{\begin{array}{l}
\text { Rudbeckia-laciniata. } \\
\text { Broad jagged leaved. } \\
\text { Rudbeckia. }
\end{array}\right.
$$

$\left\{\begin{array}{l}\text { covered with rough } \\ \text { Prominenfces. }\end{array}\right.$ Covered with fharp Fig. 9. Muricated.
Cactus-parafidica
P'oints or Prickles: 


\section{$\left[\begin{array}{lll}12 & ]\end{array}\right.$ \\ $\begin{array}{lllllll}\mathrm{T} & \mathrm{R} & \mathrm{U} & \mathrm{N} & \mathrm{C} & \mathrm{U} & \mathrm{S} .\end{array}$ \\ The SUPERFICIES.}

TOMENTOSUM.
Fig. I.
Tomentofe. $\left\{\begin{array}{l}\text { Verbafcum-thapsus } \\ \text { Moth-Mullein. }\end{array}\right\}$ covered with Down.

L A N A T U S.
Fig. 2.
Ianated: $\quad\left\{\begin{array}{l}\text { Salvia-cthiopica. } \\ \text { Esthiopian Sage. }\end{array}\right\}_{\text {woolly }}^{\text {covered with Hair, }}$

V I L L O S U M.
Fig. 3.
Villous. $\quad\left\{\begin{array}{l}\text { Inula-hirta. } \\ \text { Hairy Inula. }\end{array}\right\}$ covered with foft Hair.

P I L O S U M.
Fig. 4.
Pilofe. $\quad\left\{\begin{array}{l}\text { Saxifraga granulata } \\ \text { white Saxifrage. }\end{array}\right\} \begin{aligned} & \text { covered with Hair } \\ & \text { thinly placed. }\end{aligned}$

H I S P I I U S. $\{$ Dipfacus-laciniatus, $\}$ covered with ftiffHairs Hifpid. [Laíciniated Teafel. \}or Briftles.

A CULE A T US. "Rora-canina.

Fig. 6.

Aculeated.

Dog-Rcie.

jprickly, armed with

$\int$ Pricliles.

S I N O S US.
Fig. 7.
Spinus. $\quad\left\{\begin{array}{l}\text { Prunus-jpinofus. } \\ \text { Sloc. }\end{array}\right\}$ armed with Thorns or 


\section{(13) \\ T $R \quad U \quad N \quad C \quad U S$.}

\section{The SUPERFICIES.}

$U \mathrm{R}$
$\begin{array}{r}\mathrm{E} \text { ig. I. } \\ \text { Stinging. }\end{array}$ S. $\left\{\begin{array}{l}\text { Urtica-dicica. } \\ \text { Common Nettle. }\end{array}\right\}$ armed with Stings.

S T I PU L A T U S.
Fig. 2.
Stipulate. $\left\{\begin{array}{l}\text { Lathyrus-latifolia. } \\ \text { Everlafting Pea. }\end{array}\right\}$ having ftipule.

MEMBRANACEUS. $\{$ Fig. 2. cloathed with a Membrane.
Membranaceous.

BUL B I ER S US. [Lillium-bulbifefum: J bearing at the Axillas $\begin{array}{c}\text { Fig. 3. } \\ \text { Bulbiferous. }\end{array}$ Bulb-bearing Lily. $\} \begin{gathered}\text { the Leaves fmall } \\ \text { Bulbs. }\end{gathered}$ 


\section{( 14 ) \\ $\begin{array}{lllllll}T & R & U & N & \mathrm{C} & \mathrm{U} & \mathrm{S} \text {. }\end{array}$}

\section{The COMPOSITION.}

E E D. I S.
Without Joints. $\quad\left\{\begin{array}{l}\text { Scirpus-lacuftris. } \\ \text { Bull-rufh. }\end{array}\right\}_{\text {joints. }}^{\text {without knots or }}$ SIMPLICISSIMUS.
Fig 2.
Very Simple. $\left\{\begin{array}{l}\text { Mercurialis-perennis. } \\ \text { Dog's Mercury. }\end{array}\right\}$ with few or no Sig. 3.
Simple. $\quad\left\{\begin{array}{l}\text { Stellaria-bolofea. } \\ \text { Stichwort. }\end{array}\right\} \begin{aligned} & \text { that rifes uniform and } \\ & \text { regular to the Top. }\end{aligned}$

I $N$ T E G E R.
Fig. 4.
Intire. $\quad\left\{\begin{array}{l}\text { Ariftolochia-clematis. } \\ \text { Birthwort. }\end{array}\right\}$ undivided.

ARTICULATUS.
Fig. 5.
Articulated. $\left\{\begin{array}{l}\text { Cactus-tuna. } \\ \text { Opuntia or Indian } \\ \text { Fig }\end{array}\right\}$ joirted. 


\section{[ 15$]$ \\ $\begin{array}{lllllll}T & R & U & N & G & U & S\end{array}$}

\section{The COMPOSITION.}

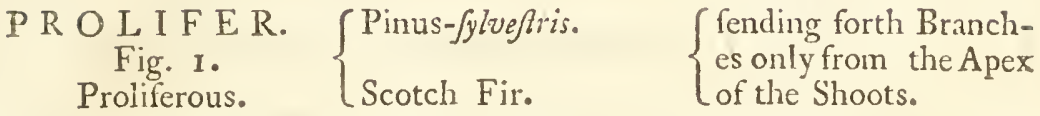

DICHOTOMUS.
Fig. 2. Vifcum-album. $\{$ branching always by

Branching doubly. (White Miftetoe. (two, like the Letter V.

BRACHIATUS. $\quad$ Clufia-flava. $\quad$ branchingoppofite.the

Fig. 3.
Brachiate. $\quad\left\{\right.$ Yellow Clufia. $\quad\left\{\begin{array}{l}\text { upper Pair crofling the } \\ \text { next below. }\end{array}\right.$

SUBRAMOSUS.
Fig. 4.
Subrameus. $\quad\left\{\begin{array}{l}\text { Saururus-cernuus. } \\ \text { Lizard's Tail. }\end{array} \quad\left\{\begin{array}{l}\text { having few lateral } \\ \text { Branches. }\end{array}\right.\right.$ Fig. 5 . Ramous. Stock July Flower. $\{$ Branches.

RAMOCISSIMUTS. Fig. 6.

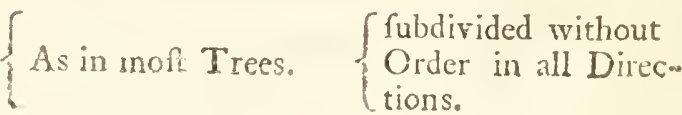




\section{$\left[\begin{array}{lll}1 & 16\end{array}\right]$ \\ T R U N G U S。 \\ The COMPOSITION.}

VIR G A T US:
$\begin{gathered}\text { Fig. I. } \\ \text { Virgated. }\end{gathered}$$\quad\left\{\begin{array}{l}\text { Blitum-virgatum. } \\ \text { Strawherry-Blite. }\end{array}\right\}$ Twith many flender

PANICULATUS. F Avena-fatua.

Fig. 2.

$\left.\begin{array}{c}\text { Fig. 2. } \\ \text { Paniculated. }\end{array}\right\}$ wild Oats.

T when the Brancises

are varioully fubdie

$\int$ vided.

FASTIGATUS.
Fig. 3.
Faftigated. $\quad\left\{\begin{array}{c}\text { Androfacefepientrio- } \\ \text { nalis. } \\ \text { Tooth-leaved } \\ \text { Androface. }\end{array}\right\} \begin{aligned} & \text { Branches arifing from } \\ & \text { a Center to an equal } \\ & \text { Height. }\end{aligned}$

P A T E N S.

Fig. 4.

Patend.

$\left\{\begin{array}{l}\text { Ancthum-foniculum } \\ \text { Fennel or Finkle. }\end{array}\right\}$ the Branches fpreading

DIVARIGATUS.
Fig. 5.
Divarigate. $\quad\left\{\begin{array}{l}\text { Coffee-arabica. } \\ \text { Coffee }\end{array}\right.$

Divarigate.

the Branches forming an ohtufe Angle from - the Trunk, 


\section{( 57$)$}

\section{R A M I.}

The Branches, are Part of the STEM.

A L T E R N A. [Aconitum-Napelles. $]$ coming out fingle, fol-

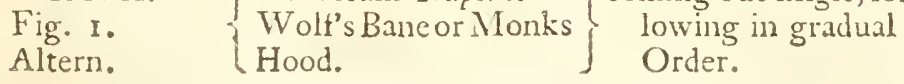

D I S T I C H I. $\begin{gathered}\text { Fig. 2. } \\ \text { Diltich. }\end{gathered} \quad\left\{\begin{array}{l}\text { Polypodiunaculeatum. } \\ \text { Prickly Polypoditm. }\end{array}\right\}$ in two Rows oppofite.

S P A R S I.
Fig. 3.
Sparfed. $\quad\left\{\begin{array}{l}\text { Rufcus-aculeatus. } \\ \text { Butchers-Broom. }\end{array}\right\}$ without any Order,

$\left.\left.\begin{array}{c}\text { CON E E T I. } \\ \text { Fig. 4. } \\ \text { Confert. }\end{array}\right\} \begin{array}{l}\text { Cupreffus-fempervirens } \\ \text { Cyprefs. }\end{array}\right\}$ crowded togrether.

$\left.\left.\begin{array}{c}\text { OP P OSIT I. } \\ \text { Fig. 5. } \\ \text { Oppofite. }\end{array}\right\} \begin{array}{l}\text { Monarda-fifulofa: } \\ \text { Ofvego Tea. }\end{array}\right\}$ 


\section{$\left[\begin{array}{lll} & 18 & ]\end{array}\right.$}

\section{R A M I.}

VERTICILLATI.
Fig. I.
Verticiliate. $\left\{\begin{array}{l}\text { Alifma-plantago. } \\ \text { GreatWater-Plantain }\end{array}\right\} \begin{aligned} & \text { Branches furrounding } \\ & \text { the Stem like the Ray } \\ & \text { of W Wheel, in a whorle }\end{aligned}$

E R E C T I.
Fig. 2.
Ered. $\quad\left\{\begin{array}{l}\text { Populus-dilatata. } \\ \text { Lombard or Po Poplar. }\end{array}\right\}$ upright, perpendicular

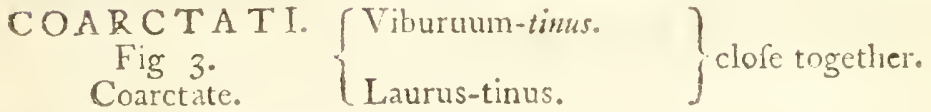

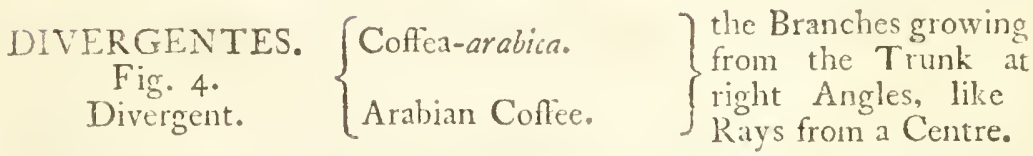

1) VARIGATI.
Fir. 5.
D.varigate. $\quad\left\{\begin{array}{l}\text { Quercus-robur } \\ \text { Oak. }\end{array} \quad \begin{array}{l}\text { the Branches fhooting } \\ \text { from the Trunk, fo as } \\ \text { tomakeobtuíe Angles. }\end{array}\right.$ 


\section{[ 19]}

\section{$\begin{array}{llll}R & A & M & \text { I: }\end{array}$}

DE F E E X I.
Fig. I.
Deflexed. $\quad\left\{\begin{array}{l}\text { Salix-babalonica } \\ \text { Weeping-Willow. }\end{array}\right\} \begin{aligned} & \text { bending downwards } \\ & \text { Archways. }\end{aligned}$

R E F L E X I.
Fig. 2.
Reflex. $\quad\left\{\begin{array}{l}\text { Clufia-flava. } \\ \text { Yellow Clufia. }\end{array}\right\}$ bending upwards to.

RETROFLEXI.
Fig. 3.
Retroflex. $\quad\left\{\begin{array}{l}\text { Rhamnus-catbarticus. } \\ \text { Buckthorn. }\end{array}\right\} \begin{aligned} & \text { bending back towards } \\ & \text { the Trunk. }\end{aligned}$

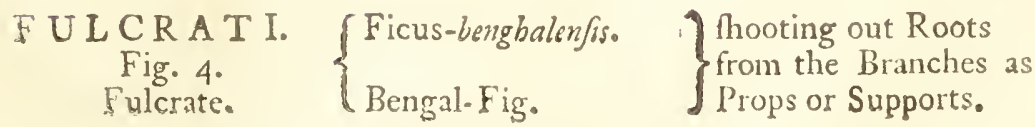


Leaves, are to be confidered in threo Refpects, Simple, Compound, and Determinate.

\section{S I M P L E L E A V E S.}

TAB. $21,22,23,24,25,26$.

They are when a Partial (Footftalk) bears only a fingle Leaf, which differs an Refpect to its Circumfoription as,

Angles, Margin, Superficies Apices and Substance.

CIKCUMSCRIPTION, confiders the Circumference without any Sinufes and Angles.

$\Lambda N G L E S$, are the prominent Part of an horizontal Leaf.

S I N U S, Hollows or Cavities that divide the Dink of a Leaf into Parts: c. g. Fig. 3. 16. 47.61 .27$.

M A R G I N, is the cxtream Boundery of a Leaf or its Sides not including the Difk of the Leaf.

SUPER FICIES, the fupinc (uppor Difk) or the Prone (under Difk) of a Leaf.

A P E X, is the Extremity of a Leaf in which it terminates.

S ITRST ANCE, of a Leaf is to be confidered according to the Condition of its Sides. 


\section{(2ï)}

\section{S I M P L E L E A V E S.}

The Organs by which Plants are put in Motion. A C E R O S U M.
Fig. I.
Acerofe. $\left\{\begin{array}{l}\text { Pinus-Syluefris. } \\ \text { Scotch-Fir. }\end{array}\right\}$

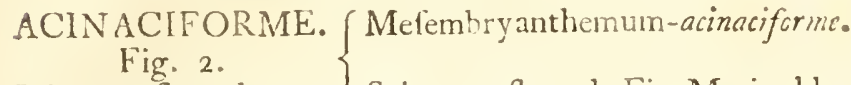
Scimetar-fhaped. Scinetar-fhaped, Fig-Marigold.

A C U LEA T UM.
Fig. 3.
Aculeated. $\left\{\begin{array}{l}\text { Solanum-mammofum. } \\ \text { Annual Barbadoes } \\ \text { Nighthade. }\end{array}\right\}$ prickly. ACUMINATUM.
Fig. 4.
Acuminated. $\quad\left\{\begin{array}{l}\text { Ficus-Religiofus. } \\ \text { Malabar-Fig. }\end{array}\right\} \begin{aligned} & \text { terminating in a tayer } \\ & \text { Point. }\end{aligned}$

A N C E P S. $\quad$ Sifyrinchium-Bermal- Form ng two oppofite Fig. 5.
Anceps. $\quad\left\{\begin{array}{l}\text { Silyrinchium-Bermul- } \\ \text { diana. }\end{array}\right\} \begin{aligned} & \text { Alyles lengthwars, } \\ & \text { the Dink being conves. }\end{aligned}$

ANGULATUS. An rnlated. $\{$ Fig 16.20.27.

B A R B A T U M. $\left\{\begin{array}{l}\text { Mefembrianthemum- } \\ \text { barbatuin. } \\ \text { Bearded Fig-Mari old. }\end{array}\right\}$ A Tuft of ftrong Hairs B I F I D U M. \{Bauhiniafcandens. Tdrided by linean $\left.\begin{array}{c}\text { Fig. } 7^{\circ} \\ \text { Bitid. }\end{array} \quad \begin{array}{c}\text { Clinbing Mountain } \\ \text { Ehony. }\end{array}\right\} \begin{aligned} & \text { Winufes and ftreight } \\ & \text { Margins. }\end{aligned}$ Trifidum, quadrifid, quinquifid, from the Number of Lobes.

B U L L. A T U M.
Fig. 8.
Bullated. $\left\{\begin{array}{l}\text { Thea-viridis. } \\ \text { Green Tea. }\end{array}\right.$ whe the Subrtance of
the Leaf rifes ligh abore the Vins, fo as to appear like little Blitters (rugofum in a greater Degree.)

CANALICULATU11. \{ Tradefcantia virginica. Having a deep ChanFig. 9 . Channelled. nel running from the Virginian spidersuort. J Bafe to the Apex. CAR IN A T UM. $\{$ Aloe dificha.
Fig. IO.
Cirrinated.

CARNOSUM. Fig. I. Carnous.

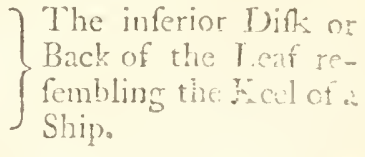
Jinternalle renlete wit? a Pulp of ficthy sile fance. 


\section{$(21)$}

\section{S I M P L E L E A V E S.}

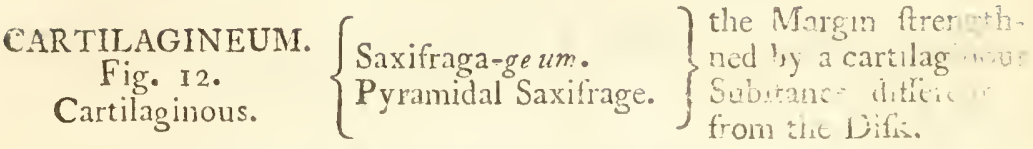

C I L. I A T U M. \{Fig. Ir. whofe Margin is guaaded by T Ciliated. $\quad$ Briftles, like Eye-lahhes.

CIR RHOSUM.
Fig. I 3.
Ciritiole. $\quad\left\{\begin{array}{l}\text { Gloriofa-fuperba. } \\ \text { Superb Lily. }\end{array}\right\}$ whi h terminat a

COLORATUM

Fig. It.

Coloured.

$\left\{\begin{array}{l}\text { Amaranthus-tricolor. } \\ \text { Three-coloured Ama- } \\ \text { rantlis. }\end{array}\right\} \begin{aligned} & \text { whe genterav } \\ & \text { of allots }\end{aligned}$

COMPRESSUM.

Fig. 15 .

Comprefied. $\left\{\begin{array}{l}\text { Mefembryanthemum- } \\ \text { difforme. } \\ \text { Fig-Marigold with } \\ \text { different Leaves. }\end{array}\right\}$

when compr on its marginal Stdes and the Subftance of the Leaf becomes greater in the Din.
CONTEXUM Fig. 16. Convex.

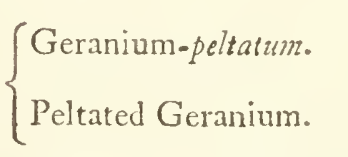

when the Margin
forms a concave Arch
with the Difk, (re-
verfe of concave.)

CONCA VUM Fig. 17. Concave.

CORTI A T U M Fing 18.

Corditcd.

$\left\{\begin{array}{l}\text { Martinia-perennis. } \\ \text { Perennual Martinia. }\end{array}\right\}$

frifing from the Margin to the Difk.

Shaped like a Heart, is ovate, hollowed at $\int$ the Bafe and is defti-
tute of Angles.

CORDA TUM

Obverfe.
Hin. In.
Obverfely cordated. $\left\{\begin{array}{l}\text { Oxalis acetofoll } \\ \text { Wood Sorrel. }\end{array}\right.$

Obverfe.
Hin. In.
Obverfely cordated. $\left\{\begin{array}{l}\text { Oxalis acetofoll } \\ \text { Wood Sorrel. }\end{array}\right.$

obverfe.
Hix. ro.
Obverfely cordated. $\left\{\begin{array}{l}\text { Oxalis acetofell } \\ \text { Wood Sorrel. }\end{array}\right.$

CORDATUM. fagittatum.

Fis. 20.

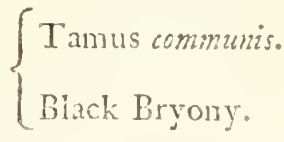

$\left\{\begin{array}{l}\text { Oxalis acetoflla } \\ \text { Wood Sorrel. }\end{array}\right.$
When a heart-fhaped Leaf is fixed by the Apex inftead of the Bafe to the Petiole (foot $\{\mathrm{a}$ k) 


\section{$\left[\begin{array}{ll}22 & ]\end{array}\right]$ \\ S I M P L E L E A V E S.}

CREN A T U M.
Fig. 21.
Crenated. $\left\{\begin{array}{l}\text { Betonica officinalis. } \\ \text { Wood-Betony. }\end{array}\right.$

CR I S P U M.
Fig. 22,
Crifped. $\quad\left\{\begin{array}{l}\text { Malva-crippa. } \\ \text { Curled Malva. }\end{array}\right.$

The Margin is cut with fmall Teeth or Angles, inclining towards neither extremity.

Undulated from the

Circumference of the Margin, being too long for the Difk

All thofe Leaves are monttrous.

E UCULA T UM. Geraneum-cuculatum. Rolled up lengthways Fig. 23.

Cuculated.

CUNEIFORME.
Fig. 24.
Cuneiform, $\quad\left\{\begin{array}{l}\text { Craffula-portulacoides. } \\ \text { Purflain-leav'd Craf- } \\ \text { fula. }\end{array}\right\}$ African Tree Cranes- $\}$ in Form of a Hood or Cawl.

Whole length exceeds the breadth gradually narrowing towards the Bafe like a wedge.

\section{$\underset{\text { Cufpidate. }}{\text { CUSPIDATUM. }\left\{\text { Fig. } 4 \cdot 43^{\circ}\right.}$}

CYLINDRACEUM. (Mefembryanthemum- ? Fig. 25.

Cylindraceous.

D) $\mathbb{E}$ D A E U M .

Fig. 26.

Dedaleous. $\left\{\begin{array}{l}\text { bipidum. } \\ \text { Hifpid Fig-Narigold. }\end{array}\right\}$

[Alftromeria pellegrina, Leaves whofe Texture \{ Beautiful Alftomeria, is remarkably beauti$\{$ and Saxifragafolonifera $\}$ ful, and exquifitely \{ Stolonifcroussaxifrage wrought.

A Cylinder or Roller.

DELTOIDEUM.
Fig. 27.
Deltoid. $\quad\left\{\begin{array}{l}\text { Mefembryanthemum } \\ \text { deltoideum. } \\ \text { Deita-Raped Fig- } \\ \text { Marigold, } \\ \text { and Sea Purfain Tree. }\end{array}\right\}$

Fig. 27.

Deltoid.

DEN TA T U M. Blitum virgam.

Fig. 28.

Dentated.

$\{$ Strawberry Blite.

DEPRESS U M.

Fig. 20 .

Deprefied.

$\left.\begin{array}{l}\text { Mefembryanthemum } \\ \text { difforme. } \\ \text { Fig-Marigold with } \\ \text { defomid Leaves. }\end{array}\right\}$

Whofe Angles are formed like the Greek Delta.

Ilaving horizontal Points ( $T$ eeth) ofthe fame confiltence with the leaves, at a little Diftance from each other.

When in the Diks is depreffed, and the Sides rifing higher than the Dik.

DOLABRIFORME
Fig. jo.
Dolabriform. $\left\{\begin{array}{l}\text { Mefembryanthemun } \\ \text { dolabriforme. } \\ \text { Hathet-fhaped, Fig- } \\ \text { Marigold. }\end{array}\right\}$ Comprefied, fubrotund, obrufe; on the under Siderour if th; (retenbling ant Axe or Hachet. 


\section{$(22)$ \\ SI M P L E L E A V E .}

ECHIN A T UN. $\{$ Ilex echinatum.

Fig. 3 I.

Echinacd.

$\left\{\begin{array}{l}\text { Hedge-hog Holly. } \\ \text { Aculi or Pricklès, }\end{array}\right.$ ELLIPTICUMT. $\left\{\begin{array}{l}\text { Whofe longitudinal Diameter fur- } \\ \text { Eliptic. }\end{array}\right.$ 59. $\begin{array}{l}\text { pares the Tranfverfe, and narrow } \\ \text { at both Extremitses. }\end{array}$

ENARGINATUNi
Emarginated. $\{$ Fig. 24.32.33. $\}$ When the Apex termi-

EMIARGINATUM acutum

Fig. $3^{2} \cdot$
Acutely emarginated. Silver Fir.

EMARGINATUM obtulum.

Fig. 33 .
Obtufelyemarginated.

$\left\{\begin{array}{l}\text { Hermannia alnifolia. } \\ \text { Alder-leaved Hermannia. }\end{array}\right.$

EMARGINATUM! utringue.

Allround 34.

E N S I F O Rinated.

$\left\{\begin{array}{l}\text { Hydrocodile vulgaris. } \\ \text { Water Navel-wort. }\end{array}\right\} \begin{aligned} & \text { When notched all } \\ & \text { round. }\end{aligned}$ Fig. 35 .

Enfiform.

$\{$ Aletris uvaria.

Like a two-edged

Sword, gradually ta$\int$ pering to the Point.

ER O S U M. $\left\{\begin{array}{l}\text { Salvia Ethiotis. } \\ \text { Jig. 36. } \\ \text { Enofe. }\end{array}\right\} \begin{aligned} & \text { When a finuated Leaf } \\ & \text { has its Marginbroken } \\ & \text { by fmaller Sinufes, as } \\ & \text { if gnawed. }\end{aligned}$

F I S S U M. $\{$ Fir. 32. 7 Divided at the Apex, its Sinus beCloven or cleft. $\quad$ Fig. $32 . \%$ ing linear and the Margin ftraight. B I F I D U M. $\left\{\begin{array}{l}\text { Fig. 2I. 7. Trifid, quadrifid, quinquifid, mul- } \\ \text { tifid, according to its Numbers. }\end{array}\right.$

$\mathrm{G} I \mathrm{~B}$ B U M. Fig. $3 \%$. Gibbous.

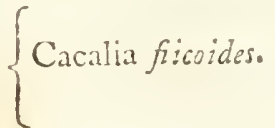

G L A BR U M. Fig. 28 . Smuoih.

Citrus aurantium.

Oruge. II A S T A T U Xi. Cuacalia fucveolens. Hfated.
When by Means of the intermediate Pulp both Surfaces are rendered convex.

When the Surface is frrooth without any Unevenefs.

Triangular, the lateral bac hollowed, the Anglestpreading : refemblims an tiaberd. 


\section{( $\left.\dot{2}_{3}\right)$}

\section{S I M P L E L E A V E S.}

Hi I R S T U M.

Fig. 40.

Hirfute.
$\left\{\begin{array}{l}\text { Alcea rofea. } \\ \text { Holly hog. }\end{array}\right.$

Rough and hairy in tle $\int \mathrm{DiR}$.

Hifpit.

Fig. 25. Covered irregularly with fragil (

I N T E GR U M. Integer or Intire.

L $\mathbb{E}$ V I S. Fig. 41 . Smooth.

LACERATUNI. Fig. 42, Lacerated.

I A N A T U M. Lanated. LANCEOLATUM.
Fig. 43.

Lanceolated. $\{$ Fig. $38 \cdot 41 \cdot 51 \cdot 55$

Where the Nargin is

Orchislatifulia. When the Superficies intire, willout any Crenes or Notches. \{Broad-leaved Orchis. $\}$ is my Unevenefs. Scnecio hierachifolia. Thofe Margin is vaAmerican Groundfel. $\left\{\begin{array}{l}\text { regular Segments: } \\ \text { (as if rent or torn) }\end{array}\right.$ Fig. $3^{5}$. Corered as with a Spider's Webib. Dogs-bane.

ls oblong, but trat dually tapering towards the Extremitv, and icrminating in a Point.

L. ACIN A T UM. Fig. 44. Lacinated.

$\left\{\begin{array}{l}\text { Delphixiun clatum. } \\ \text { Bee Larkfpur. }\end{array}\right\}$

L INEARE。 Fig. 45. Linear.

LINEATUMT.

Lincated.

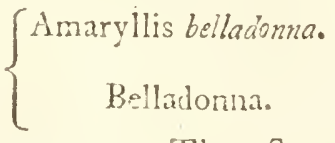

Divided firft into Lacinix and thofe again irregularly cut into fmaller segments.

The lateral Miargins parallel, narrowing a little towalds the Apex.

Tlue Superficies nightly ftreaked Fig. I 3. longitudinally with parallel Lines, not imprefing the Surface

LINGUIFORME.
Fig. 46.
Linguiform. $\left\{\begin{array}{l}\text { Aloe dificha. } \\ \text { Liftich Aloe. }\end{array}\right.$ Is linear and carnous, obtule on the under Side convex; iometimes the Niargin is cartilaginous : (Tonerto fhated.)

L O P A T U M. Fig. 4\%. Lobated. $\left\{\begin{array}{l}\text { Acer compeftris. } \\ \text { Common Maple. }\end{array}\right.$ Is divided to the Middle into difkant Parts, haring their Margins convex.

Bilobum, trilobum, quatrilobum, quinquelobium, 2. 3. 4. 5. iobum, rom its Numbers of Lohes.

LUNULATUII.

Fig. 48 . Lunated. $\left\{\begin{array}{l}\text { Paffifora Mitsicuja. } \\ \text { Paffion-tlower of Do- } \\ \text { mingo. }\end{array}\right\} \begin{aligned} & \text { Subrotund holiowed, } \\ & \text { at the Bafe lihed a } \\ & \text { Crefeent. (Moon- } \\ & \text { Bropedo) }\end{aligned}$ 


\section{SI M L E L E A V S S.} I. IT C I D U M. $\left\{\begin{array}{l}\text { Laurus nobilis. } \\ \text { Fig. 49. }\end{array}\right.$ Bright, Inining, glofy's Luciel.

IIACULATUM.

Common Bay. (reflecting Light.) Marked with spots of a different

Maculated. $\left\{\begin{array}{l}\text { Fig. 4. Colour, hut fmoth and even with } \\ \text { the Sulface of the I eaf. }\end{array}\right.$

Divided tranfverfely

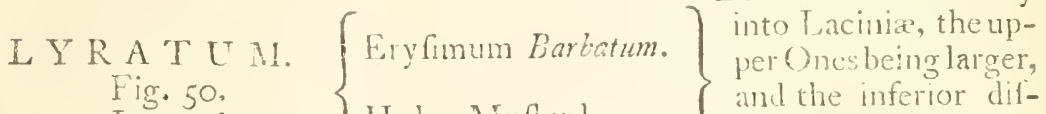
Ly rated. \{Herge Muftart. $\}$ and the inferior diltant from each other. (Lyre-fhaped.)

MemiranaCEUM. Cama Indica. Fig. 5 r.
Nembranaceous. $\left\{\begin{array}{l}\text { Indian Reed: } \\ \text { M C }\end{array}\right.$ Having no dittinguifh. able Pulp between the $\int$ two Surfaces,

MIUCRONATUM. Riucronated. MUCRONATUM. acutum.

Fig. 52.

Acutely mucronatad. MUCRONATUM. cobtufun.

Fig. $53^{\circ}$

Fig. 53. MLLTIPAR TITUM. Fig. 54 . Miultipartite. NERVOSU MI. $\{$ Fig. 52. 53. Tenninating in a flarp Point. $\{$ Statice tatarica.

Tartarian Thrift. A Afarum Canadenfe. Canadian Afarbecca. Aconitum napel'us. \{ ivolfs-bane or Nionks-bood.

$$
\begin{aligned}
& \text { Alifma planingo. } \\
& \text { Grat Water-Plan. } \\
& \text { tain. } \\
& \text { Fig. 4. } 49 \cdot 3^{8}
\end{aligned}
$$

When a I eafis divider? into miny Segments. The Norves cxtending from the Bate to the Apex.

$\left\{\begin{array}{l}\text { Euphorbia latbroides. } \\ \text { Burning thorny Plant } \\ \text { or Spurg. }\end{array}\right.$

O B T U S U M. Piper obtuftolia. Fig 57. Obtule.

ORBICULA TUM Fig. 58

Oibicular. $\left\{\begin{array}{l}\text { Pepper with ohtule } \\ \text { Leares. }\end{array}\right.$ $\left\{\begin{array}{l}\text { Tropreolum minus. } \\ \text { Indian Crels. }\end{array}\right.$
Thofe Jongitudinal Dinmeter is feveral Times longer than the Tranfverfe, both Extremities being rounded. butnarrower than the Segments of a Circle.

Which tuminates at the $A$ pers, rounded in a Maniner lefs than at Circle.

Forming a Circle. 


\section{$(24)$ \\ S I M P L E L E A V E S.}

O V A L, E. [Mamea Ameriana $]$ Whofe longitudinat

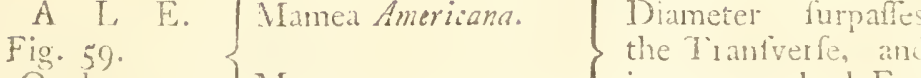
Oval. Mame. $\quad\} \begin{aligned} & \text { is narrow at both Ex- } \\ & \text { tremities }\end{aligned}$

O V A T U M. Fig. 60. Ovate.

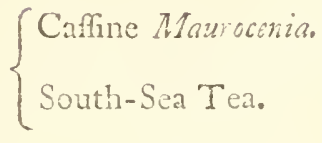

Whofe longitudinal Diameter is longer than the 'Tranfverie, and the Segment of the Bare circumferbing a Circle; the Apex of the fame Form, but narrower.

OVALE oblongum. $\int$ Fig. 40. Its longitudinal Diameter is greater Oblong oval. $\{$ Fig. 49. than the Oval.

O V A T U M. obverfum. Fig. 6I. Obverfe, Ovate. $\left\{\begin{array}{l}\text { Samolus valcrandi. } \\ \text { Round-leaved Water } \\ \text { Pimpernel. }\end{array}\right\} \begin{aligned} & \text { Whofe Bafe is narrow, } \\ & \text { anovate Leaf invert- } \\ & \mathrm{el} .\end{aligned}$ PALMATUM. Fig. 62. Palmated.

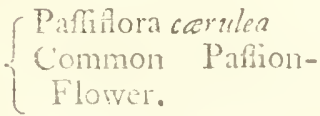

PANDURATUM. vel. Panduriforme. Fig. 63. Panduriform. Fiddle Dock.

A Leaf with feveral Divifions divided almoft to the Bafe.

Is oblong, hroad below, narrowed at the Sides.

$\int$ (Formed like a Spanim Guittai.)

PAPILOSUM. Fig. 64. papilole.

PAPULOSUM. Fig. 65. Papulole $\left\{\begin{array}{l}\text { Mefembryanthemum } \\ \text { chryfthallinum. } \\ \text { Diamond Ficoidea. }\end{array}\right.$ $\{$ Echium.

Whofe Surface is covered with Dots or little Biaduers,

PORABOLICUM Fig. 66. l'orbolic.

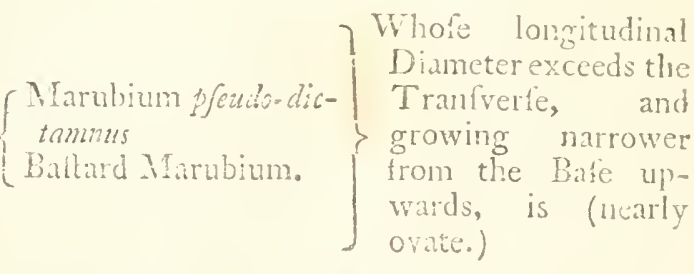




\section{$24)$ \\ S I M P L E L E A V E S,}

PENTANGULARE.
Fig. 67.
Pentangular. $\left\{\begin{array}{l}\text { Geranium peltatume } \\ \text { Peltated Cranes-Bill }\end{array}\right\} \begin{aligned} & \text { With five prominen: } \\ & \text { Angles furrounding } \\ & \text { the Difk. }\end{aligned}$

PERTU UN. $\quad\left\{\begin{array}{l}\text { Draconticumpertum. } \\ \text { Perforated Draconti- }\end{array}\right\}$ As if Holes were cut

Fig. 68.

Perforated.
P I L O S U M. Fig. 69. Pilous.

PINATIFIDUM.

Fig. 70.

Pinatifided.
Perforated Dracontium. through the Dilk.

Covered with diftinct

\}long Hairs.

Common creeping

Moufes-Ear.

Centaurea-calcitras. Is traverfely divided $\left\{\begin{array}{l}\text { Pinatified Star-1 thif- } \\ \text { tle. }\end{array}\right\} \begin{aligned} & \text { into long horizontal } \\ & \text { tranfrerte Lacinix. }\end{aligned}$

$P \mathrm{~L} A \mathrm{~N} U \mathrm{M}$. Fig. 7 I. Plaine.

\section{Ruicus androgynis. \\ Broad leafed Rufcus.}

PLICA TUM.

Fig. 72 .

Plicateri.

PKAMORSUM.

Fig. $7 \hat{3}$.

Prxmolle.

DUITERATUM.

Fig. 74 .

T'ulverated.

PUNCTATUAI.

Fig 75 .

Punetated.

Teratrum album.

White Hellebore.

When plaited like a

Plain, flat, neither condex nor concave.

PRANGLLARIS.

lig. 70.

Qundrangu!nr.

nUTMOUANGILARIS.

Cutapuanouia. frig. 6\%

or P'entangular.

anclalobatem.

$\mathrm{I}: \mathrm{m} . \mathrm{C}^{\circ}$

acingrotus
Chamerops miilis.

Suricula Pulecrasa.

Porydercel suricula.

$\int$ Fan.

(Hypenicum ferforatum.

St. Tohn's-Trort.

obtufe as if bitten off at the Apex.

Covered with a farma or mealy Duft.

Befprinkled with ho\}low Dots or l'oints.

With four prominent Com Furfe-Tail f Angles. f Acol Pouliz piatanus.
S.comone $1 \mathrm{xe}$
Hung fire Angles. 


\section{(25)}

\section{S I M P L E L E A V E S.}

R E C T U M.
Fig. 78.
Straight. $\left\{\begin{array}{l}\text { Sarracenia Flava. } \\ \text { Yellow Side-Saddle } \\ \text { Flower. }\end{array}\right\} \begin{aligned} & \text { Straight, Stiff and } \\ & \text { Ereet. }\end{aligned}$ REN IF OR M E.
Fig. 79.

Reniform.

Afarabacca.

Subrotund, hollowed at the Bafe, without Angles (refembling a J Kidney.)

REPANDUM. Fig. 80. Repanded. $\left\{\begin{array}{l}\text { Tropeolum minus } \\ \text { Indian Crefs. }\end{array}\right.$ R E T U S U M. Fig. SI. Ketufe.

$\left\{\begin{array}{l}\text { Clufia flava. } \\ \text { Yellow clufia. }\end{array}\right.$

Having a ferpentine Margin without any
Angles.

Terminating in an ob$\int$ tule Sinus.

RHOMBOIDEUM. (Sida Rhomboideum. Fig. 82 . Rhomboid. Smaller upright MIal-

Quadrangular, of which the two lateral Angles are leaft. R U G O S
Fig. 83.
Rugole. M. $\left\{\begin{array}{l}\text { Salvia officinalis } \\ \text { Common Sage. }\end{array}\right.$

From Contraction of the Veins the Sub-

ftance of the Leaf rifes above the Veins, (lefs than Bullatum.)

RUNCINATUM.
Fig. 84.
Rumcinated. $\quad\left\{\begin{array}{l}\text { Leontorion taraxacum. } \\ \text { Dandelion. }\end{array}\right\} \begin{aligned} & \text { Doubly and unequally } \\ & \text { ferrated, and the An- } \\ & \text { glespointing contrary } \\ & \text { to the Serratures. }\end{aligned}$

SAGITTATUM. \{ Sagittaria fagitif folia. Fig. 85 . Sagictated Arrow Head.

Triangular, hollowed at the Bale, furnifhed $\int$ with Angles. S C A B R U M.
Fig. 86.
Scaber. $\quad\left\{\begin{array}{l}\text { Humulus lupulis. } \\ \text { Hops. }\end{array}\right.$

SERRA T U M. acuitum. Acutely lerrated. $\left\{\begin{array}{l}\text { Mercurialis perennis. } \\ \text { Dogs Mercury. }\end{array}\right\}$ Befet with little Tubercles on the Din, which are rough.

S I. R R A T U M. obtutum. Dhturely lerrated. $\left\{\begin{array}{l}\text { Ballota nigra. } \\ \text { Stinking Horelound. }\end{array}\right\}$ Its Angles obtufe. 


\section{$(25)$}

\section{S I M P E L E A V E S,}

SERR A T U M. duplicato.

Fig. $89 \cdot$
Duply Serrated. $\left\{\begin{array}{l}\text { Rubus frutitcofus. } \\ \text { Bramble. }\end{array}\right.$

S E R R A T U M.

retrofum.

Serrated backward.

SERTCEUM. Fig. ço. Silky.
Fig. 84. $\left\{\begin{array}{l}\text { Protea argentea. } \\ \text { Silver Tree. }\end{array}\right.$
When the greater Ser-
ratures are ferrated
again with fmaller
Teeth. $\left\{\begin{array}{l}\text { The Surface remark- } \\ \text { ably foft, covered } \\ \text { with filky Hairs or } \\ \text { Dowil. }\end{array}\right.$
SETACEUM Fig. gr. Setaceous.
Shaped like Briftles. Afparagus.
SI N U A T U M. Fig. 92. Sinuated.
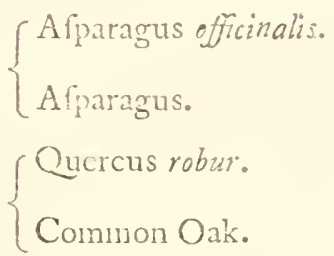

Whofe lateral Sinufes $\int$ are much dilated.

SPATULATUMI. Sempervirumcanariense. Thofe Figure is rounFig. 93 . Spatulated. Canary Houfeleck. $\quad\}$ difh, but lengthened

STR I GOSU M. $\{$ Fig. 25. i. e. Hiipidum.

STR I I U M.
Fig. 94.
Striated. $\quad\left\{\begin{array}{l}\text { Scirpus maritimus. } \\ \text { Round-rooted Baftard } \\ \text { Cyperus. }\end{array}\right\} \begin{aligned} & \text { On its Surface with } \\ & \text { channelled Streaksor } \\ & \text { Grooves, rumning } \\ & \text { lengthways parallel. }\end{aligned}$ S PINOS U M. $\left\{\begin{array}{l}\text { Acarthus fingfus. } \\ \text { Fig. aj. }\end{array}\right\} \begin{gathered}\text { Running out intohard } \\ \text { or rigid Spines or }\end{gathered}$ Spinus. $\{$ Prickly Bears-bieach. $\} \begin{aligned} & \text { or rigid Spines } \\ & \text { ftinging Prickles. }\end{aligned}$ SCIAMOSUTL Fig.có.

Squamos or Scaly, $\left\{\begin{array}{l}\text { Alce-dificha } \\ \text { Diftich Aloc, }\end{array}\right.$ Covered with Scales: ¿IRICTUM. Suant? Fig. 73. 


\section{$\left[\begin{array}{ll}26 & 6\end{array}\right]$}

\section{S I M P L E L E A V E S.}

SUBROTUNDUM.

Fig. 97.
Subrotund.

SUBULATUM.

Fig. 98.

Subulate

$\left\{\begin{array}{l}\text { Mefembryanthe:num } \\ \text { bicolor. }\end{array}\right.$
SU I Fig. 99 . Sulcated.
Nearly circular in the

$\int$ Circumference

Gradually tapering towards the Apex, fhaped like an Awl.

Which is longitudinally channelled with numerous Angles, and many interjected Sinufes.
T E R E S.

Round.

TOMENTOSUM.
Fig. I00.

Tomentofe.

$\{$ Fig. 103.25. $\left\{\right.$ Great WhiteMillein. $\left\{\begin{array}{l}\text { are interwoven and } \\ \text { parallel, diftinguifh- } \\ \text { able. }\end{array}\right.$

TRIANGULARE. Fig. 101.

Triarigular.
Coveredwith awhitifh Down, whole Hairs able.

With three prominent Angles round the Dik.
TRIGONUM vel. Fig. 102. Triquater.

Three-fided.

TRILOBATUM. Fig. 103. Trilobus.

TRUNCATUM.

Fig. 10.4. Truncated. $\left\{\begin{array}{l}\text { Atriplex halamus. } \\ \text { Sea Purflain-Tree. }\end{array}\right.$

$\left\{\begin{array}{l}\text { Butomus umbellatus. } \\ \text { Flowering Rufl. }\end{array}\right\} \begin{aligned} & \text { Whofe three Siles are } \\ & \text { equalinaniwl-thaped } \\ & \text { Leaf. }\end{aligned}$
Is divided to the Middle into three diftant Parts with the Mar. gin convex.

TUBULOSUM. (Alliun ceta

Fig. 105.

Tubulous.

$\left\{\begin{array}{l}\text { Leriodendron Tute- } \\ \text { ferc. } \\ \text { Tulip Tree. }\end{array}\right.$

Haring the Apex trum.

cated (as if cut off.)

When cuttraniverfely

Common Orion.

is bollow wilhin. 


\section{$(26)$}

\section{S I M P L E L A V E S.}

VE N O S U M. T Tamus communis. $\quad \begin{aligned} & \text { When Veins branch } \\ & \text { andmeet over the }\end{aligned}$ $\begin{array}{l}\text { Fg. 106. } \\ \text { Veinos. }\end{array}$ Black Bryony. $\}$ and are plain to the Fig. 107. Vilcid

Vifcofum. Cotton or ftinking
Groundfel.

Umbilicated.

When at the Infertion

UMBILICATUM. $\{$ Fig. 16.80. $\quad\} \begin{aligned} & \text { of the Peduncle of a } \\ & \text { Leaf, forming in the }\end{aligned}$

$\int$ middle a Cavity like

UNCTUOSUM.

Clammy or
Unctuous. Fig. 107. U E $\mathrm{E}$ S.
Fiğ. Io8.
Stinging. $\quad\left\{\begin{array}{l}\text { Urtica dioica. } \\ \text { Common Nettle. }\end{array}\right\} \begin{aligned} & \text { Furnilhed tith fragil } \\ & \text { Stimuli that arefting- } \\ & \text { ing or burning. }\end{aligned}$

UNDULA T UM.
Fig. rog.
Undulated. $\left\{\begin{array}{l}\text { Aletris capenfis. } \\ \end{array}\right.$

Is when the Dink of a

$\left\{\begin{array}{l}\text { Leaf rifes and falls } \\ \text { convexly (or }\end{array}\right.$

convexly (or Wa-
ving) towards the
Margin.

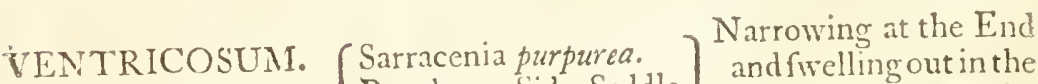
Fig. I 10. Ventricole. $\left\{\begin{array}{l}\text { Sarracenia purpurea. } \\ \text { Purple Side-Saddle }\end{array}\right.$ Middle, hollowith in 


\section{$(2.7)$}

\section{DETERMINATE LEAVES.}

Their Character is not to be taken from their own proper Structure, but from a Caufe foreign thereto; and is to be taken from their Flace, Situation, Infertion and Direction.

The Piace, is the Part where it is fattened to the Plant. The Situation, is the dippofition of the Laves on the stem. 'The Infertion, Leaves are generally inferted at their Eafe. The Direction, Leaves are general!y inferted at their Bafe.

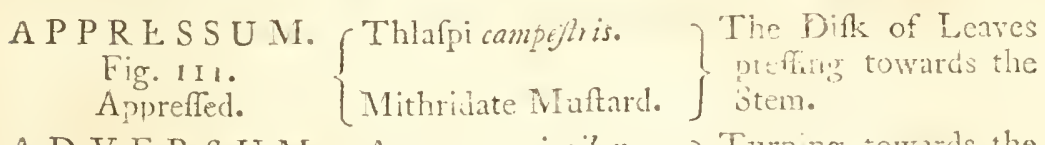
A D VER S U M.
Fig. I12,
Adverfed. $\left\{\begin{array}{l}\text { Amomum zingibir. } \\ \text { Ginger. }\end{array}\right\} \begin{aligned} & \text { Turning towads the } \\ & \text { South (not tow } \\ & \text { the Sky.) }\end{aligned}$

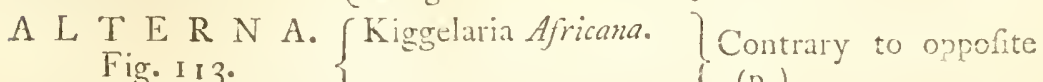
$\begin{array}{l}\text { Fig. I r 3. } \\ \text { Alternate. }\end{array}$ African Kiggelaria. $\}(\mathrm{p}$.

AMPLEXICAULE. Veratrum Aibum. $\quad$ The Bafe intirely furFig. I I.
Amplexicaul. $\quad\left\{\begin{array}{l}\text { White Hellebor. } \\ \text { rounding the Stem } \\ \text { tranivertely. }\end{array}\right.$

APROXIMATA.
Fig. 115.
Aproximate. $\quad\left\{\begin{array}{l}\text { Taxus baccifora. } \\ \text { Berry-bearing Yew. }\end{array}\right\} \begin{aligned} & \text { Many Leaves occupy } \\ & \text { the Branch, io as to } \\ & \text { leave forcelv any } \\ & \text { Space beiween them. }\end{aligned}$ ARTICULATUM.
Fig. iı. Equifetum arvenfe. $\quad\left\{\begin{array}{l}\text { When one Leaf grows } \\ \text { out ot another. }\end{array}\right.$ Fig. II6.
Articulated.

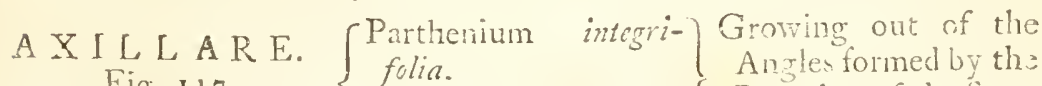
Fig. 117 . Axillary. \{ Parthenium with intire Branches of the Stem (R.)

BIF A R I A M.
Fig. II8.
Bifarious. $\quad\left\{\begin{array}{l}\text { Pinus Picea. } \\ \text { Silver Fir. }\end{array}\right.$ $\left\{\begin{array}{l}\text { The I.eaves diverging } \\ \text { (or pointing two } \\ \text { ways.) }\end{array}\right.$ H 


\section{DETERMINATE LEAVES.}

B I N A.
Fig. I Ig.
Two. $\quad\left\{\begin{array}{l}\text { Pinus Sylueftis. } \\ \text { Scotch Fir. }\end{array} \quad\left\{\begin{array}{l}\text { Growing in Pairs fromt } \\ \text { the fame Point. }\end{array}\right.\right.$

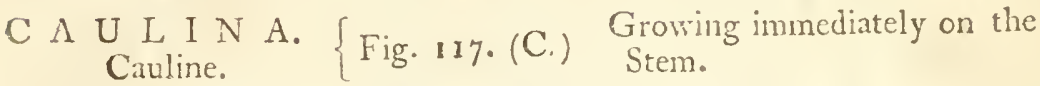

C O M A. $\quad\left\{\begin{array}{l}\text { Fritelaria imperalis } \\ \text { Fig. I20. } \\ \text { A Tuft. }\end{array} \quad \begin{array}{l}\text { Is compofed of a Num- } \\ \text { ber of Leaves clofe to- } \\ \text { gether, and termina- } \\ \text { ting the Stem. }\end{array}\right.$

CONFERT,UM.
Confert.

CONNATUM. $\quad$ Dipfacus laciniatus. $W^{\prime}$ hen two oppofite Fig. 121. $\quad$ L Leaves unitc, fo as to Fig. I21. $\quad$ Laciniated Teafel.
Lonate.
of one Leaf.

DECURRENS. C Campanula decurrcns. $\begin{aligned} & \text { The Bafe of a Seffil } \\ & \text { Leaf extending down, }\end{aligned}$ Peach-leaved Campa- $\}$ wardsalong the Stem1)ecurrent. $\quad\left\{\begin{array}{l}\text { nula. } \\ \text { Termination of the }\end{array}\right.$ Leaf.

DECUSSATUM. $\quad$ Melittis melifiphllum. $\left\{\begin{array}{l}\text { Growing in Pairs op- } \\ \text { pofite, each Pair }\end{array}\right.$ Fig. 123. $\{$ ing alternately on opDecuffate. Ofwego Tea. $\}$ polite Sidcs of the IE MER S U M. $\left\{\begin{array}{l}\text { Hottonia paiufris. } \\ \text { Fig. 124. }\end{array} \quad\left\{\begin{array}{l}\text { When funk helow the } \\ \text { Surface of the Water }\end{array}\right.\right.$ Demerfed. it ater Tio Surface of the $W$ ater 


\section{$28)$}

DETERMINATE LEAVES.

DEPENDENS.
Fig. I25. Hedyfarum motanum. $\begin{array}{l}\text { Fig. 125. } \\ \text { Dependent. }\end{array}$ Moving Hedifarum. $\}$ the Ground.

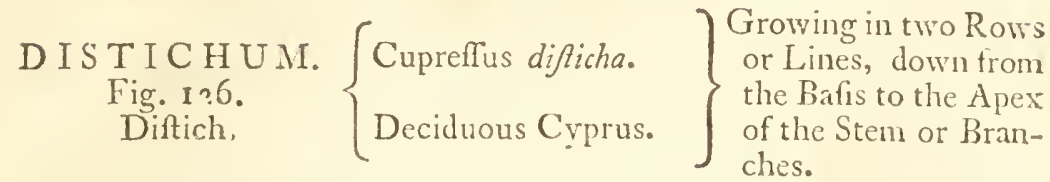

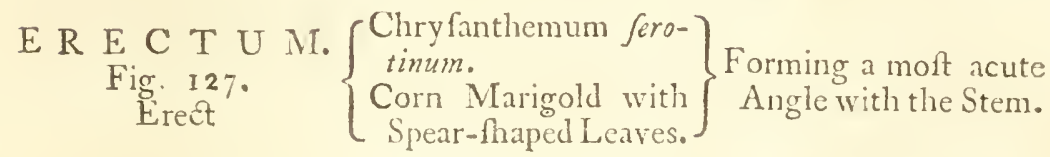
F R $\begin{array}{r}\mathrm{O} N \\ \text { Fig. } 128 \\ \text { Frons. }\end{array}$ S. $\left\{\begin{array}{l}\text { Polypodiun marginale. } \\ \text { Fern Polypodium. }\end{array}\right\} \begin{aligned} & \text { A Species of Trunk, } \\ & \text { compofed of a Branch } \\ & \text { and a Leaf blended } \\ & \text { together, and fre- } \\ & \text { quentiy united with } \\ & \text { the Fructification. }\end{aligned}$

FASCICULATUM
Fig. I29.
Fafciculated. $\quad\left\{\begin{array}{l}\text { Pinus larix. } \\ \text { Larch Tree. }\end{array} \quad\left\{\begin{array}{l}\text { Mary Leaves growing } \\ \text { in Bunches out of } \\ \text { the fane Point. }\end{array}\right.\right.$

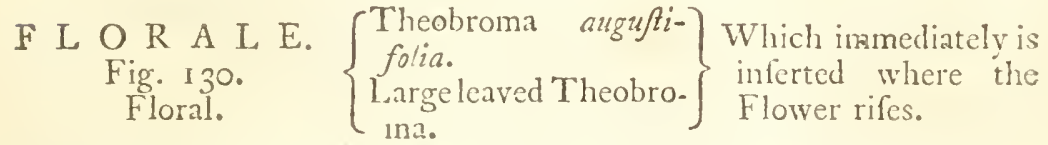

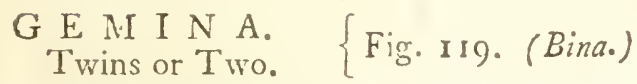

HORIZONTALE.
Horizontal. $\{$ Fig. I22. I2 3

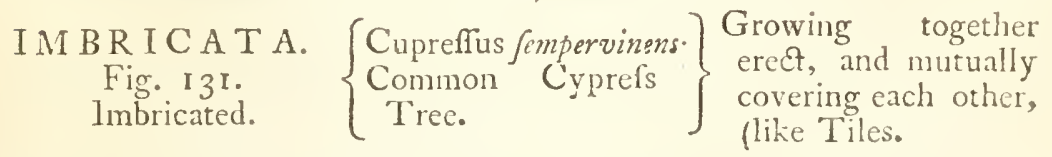

I N F L E X U M.
Fig. I32.
lnfex. $\left\{\begin{array}{c}\text { Metembryanthemum } \\ \text { calomiforme. } \\ \text { Calamiform Fig Mari- } \\ \text { gold. }\end{array}\right\} \begin{aligned} & \text { Bending inwards the } \\ & \text { Stem. }\end{aligned}$

N A T A N S.
Fig. I 33.
Natant. $\quad\left\{\begin{array}{l}\text { Potamogeton natans } \\ \text { Broad-leaved Pond- } \\ \text { weed. }\end{array} \quad\left\{\begin{array}{l}\text { Swimming on the Sul- } \\ \text { face of the Water. }\end{array}\right.\right.$ 


\section{$\left[\begin{array}{ll}28 & ]\end{array}\right.$}

\section{DETERMINATE LEAVES.}

OB L I QU U M.
Fig. 1 34.
Oblique. $\left\{\begin{array}{l}\text { Fritelaria perfica. } \\ \text { Checquered I I ulip. }\end{array}\right\} \begin{aligned} & \text { The Apex of the Leaf } \\ & \text { points to the Horizon, } \\ & \text { and at the Bafis up- } \\ & \text { wards. }\end{aligned}$

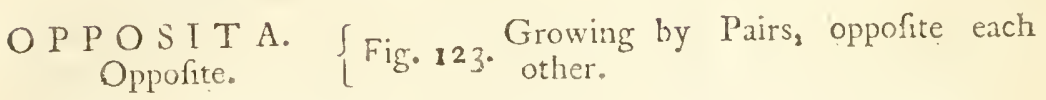

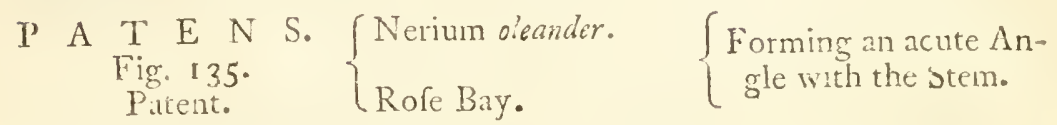

PATENTISSIMUS $\{$ Fig. 123.
Spreading wide.

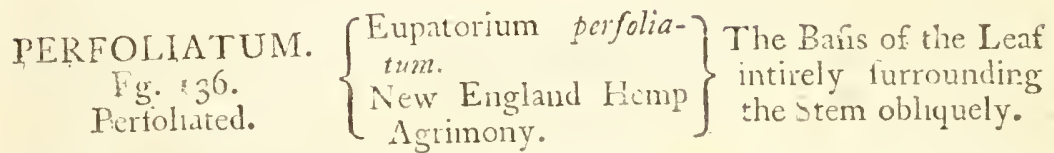

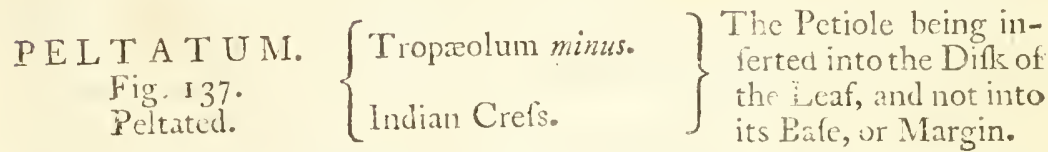

PETIOLA TUM. Pctiolated.

PIXIDA T U: M. lixidate or Box-like. PROCUMBENS.
Fig. I $3^{8}$
Procumbent. $\left\{\begin{array}{l}\text { Leontodon taraxacum. } \\ \text { Dandelion. }\end{array}\left\{\begin{array}{c}\text { Lying horizontally on } \\ \text { the Ground. }\end{array}\right.\right.$
Growing an a Footfalk at the $\{$ Fig. 125.133 Growing ana Foot $\{$ Fig. 116. When one Leaf is, as let into aro- 


\section{( 29 )}

\section{DETERMINATE LEAVES.}

QU A T E R N A.
Fig. $139 \cdot$
Quatern. $\quad\left\{\begin{array}{l}\text { Cucubalus fellatus. } \\ \text { Virginian Clove Lych- } \\ \text { nis. }\end{array}\right\} \begin{aligned} & \text { Are a Species of ftel. } \\ & \text { lated Leaves. }\end{aligned}$

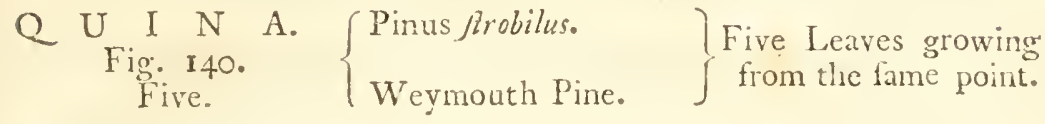
R A D I C A L E.
Radical. Fig. I $3^{8}$. Rifing immediately from the Root. R A D I C A N S.
Fig. I 4 I.
Radicant. $\left\{\begin{array}{c}\text { Afplenium rhizopbyl- } \\ \text { lum. }\end{array}\right\} \begin{gathered}\text { Pending to the Earth } \\ \text { and itrikes Root. }\end{gathered}$ R A M E U M. $\left\{\right.$ Fig. I17. (R.) and Fig. 146. $\begin{array}{c}\text { Growing on the } \\ \text { Branches. }\end{array}$

RECLINATUS.
Fig. I42.
Reclinug. $\quad\left\{\begin{array}{l}\text { Blitum virgatum. } \\ \text { Strwberry Blite. }\end{array}\right\} \begin{aligned} & \text { Bending downwards, } \\ & \text { to that the Apex of } \\ & \text { the I eaf is lower than } \\ & \text { the Bafe. }\end{aligned}$ REFLEXUM.
Reflexed.

RESUPINATUM. $\left\{\begin{array}{l}\text { Fig. I } 43 \cdot \\ \text { Refupinate. }\end{array}\right\} \begin{aligned} & \text { The Difk of the upper } \\ & \text { Side of the Leaf faces } \\ & \text { the Earth, and lower } \\ & \text { or ander Difk faces } \\ & \text { the Sky. }\end{aligned}$ REVOLUTUMI
Fig 1 44.
Revolute $\quad\left\{\begin{array}{l}\text { Dianthus barkatus. } \\ \text { Sweet William }\end{array}\right\}$ Rolled back downSEM I N A L E.
Fig. I 45.
Scininal. $\quad\left\{\begin{array}{l}\text { Raphanus. } \\ \text { Raddifh. }\end{array}\right.$ $\left\{\begin{array}{l}\text { Which before was the } \\ \text { Cotyledon and ap- } \\ \text { pears firf from out of } \\ \text { the Ground. }\end{array}\right.$ SEMIAMPLEXICAULE
Semiamplexicaul. $\{$ Fig. II2. Surrounding the Stem halfiray. S E S S I L E. $\begin{cases}\text { Fig. I 43. 146. } & \begin{array}{l}\text { Growing immediately on the } \\ \text { Stem without any Petiole or } \\ \text { Fostfols. }\end{array}\end{cases}$ 


\section{('29)}

\section{DETER MINATE LEAVES.}

S P A R S U M.
Fig. 146.
Sparfed. $\quad\left\{\begin{array}{l}\text { Polygala myrtifolia. } \\ \text { Myrtle-leaved Poly- } \\ \text { gala }\end{array}\right\} \begin{aligned} & \text { When Leaves are nu- } \\ & \text { merous on the Plant, } \\ & \text { but not in a regular } \\ & \text { form. }\end{aligned}$

$S \quad \mathrm{E} \underset{\text { Six. }}{\mathrm{N}}$ A. $\quad\left\{\begin{array}{l}\text { Rubia tinclorum } \\ \text { MTadder. }\end{array}\right.$

1 Are Species of the Itel\} lated Leaves.

T E. $\underset{\text { Three }}{\mathrm{R}} \mathrm{N}$ A. \{Fig. I35.

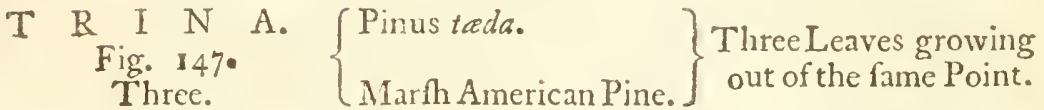

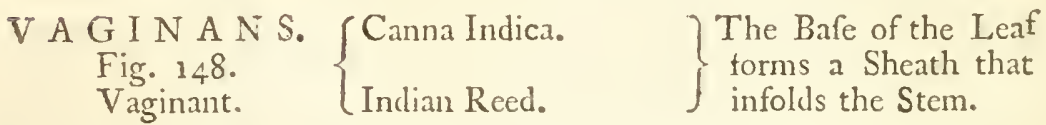

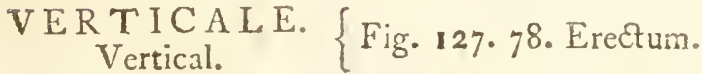

VERTICILLATUM. [Rubia Tinftorum. T Surrounding the Stem Fig. I 14.
Verticillated. $\quad\left\{\begin{array}{l}\text { like the Spokes of a } \\ \text { Wheel. (a Whorle.) }\end{array}\right.$ 


\section{(30) \\ COMPOUND LEA VES.}

Compound Leaves Signify when there are more than one Leaf upon a Petiole or Footftalk; and they are to be confidered as to Structure and Degree.

The Structure regards the Infertion of the Folioles. The Degree regards the Subdivifion of the common Petiole. ARTICULATUM. [Equifetum arvenfe. Then one Leaf grows Fig. 150. Articulated. \{Con Horfe-tail. $\}$ another.

BIGEMINATUM.
Fig. $15^{\mathrm{I}}$.
Bigeminated.

Mimofa-unguis cati.

$$
\begin{aligned}
& \text { A dichotomous or for- } \\
& \text { ked Petiolus, having } \\
& \text { two Leaves on the } \\
& \text { Apex of each Divi- } \\
& \text { fion. }
\end{aligned}
$$

\section{B I N A T U M. Fig. i 52 . Binated.

$$
\{\text { Gipfophylla fabago. }
$$

Is one of the digitated Leaves with two Foli-
oles only.
BI-PINNATUM-
CUM IMPARE.
Fig I54.
Bi-pinnated with an $\left\{\begin{array}{l}\text { Guilandina dioica. } \\ \text { Canada Nicker-Tree. }\end{array}\right\} \begin{aligned} & \text { Is when the Wings } \\ & \text { terminate with an } \\ & \text { odide. }\end{aligned}$ odd Foliole.
When the Petiolus is pinnated by lateral pinnated Wings, end. ing without a termi- nating Foliole.

Fig 153 .
Bi-pinnated-abrupt.

$\left\{\begin{array}{l}\text { Guilandina pontucella. } \\ \text { Yellow Nicker-Tree. }\end{array}\right\}$ 


\section{[ 30 ]}

\section{COMPOUND LEA VES.}

CONIUGATUM. Fig. 157.

Conjugated.

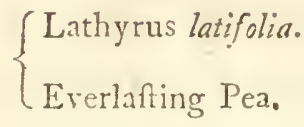

DECOMPOSITUM.

Fig. 158.

Decompofite.

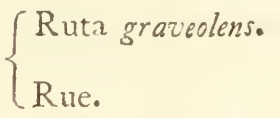

$\left\{\begin{array}{l}\text { Is when two Folioles } \\ \text { are on each Petiolus, } \\ \text { but not more. }\end{array}\right.$

When a Petiolus once divided connects many Folioles on it.

D E F O R M E. P Pronia officinalis. ] When Folioles of difFig. 159. $\{$. $\}$ ferent Figures are on Deformed. Piony of the Shops.

D I G I T A T U M.
Fig. 160.
Digitated. $\quad\left\{\begin{array}{l}\text { Vitis agnus-cajius. } \\ \text { Chaftc-Tree. }\end{array}\right.$

When more than one Leaf is connected at the Extremity of one Petiole.

GEMINATUM.

Fig. 161 .

Geminated.

$\left\{\begin{array}{l}\text { Pinus Syluefris. } \\ \text { Scotch Fir. }\end{array}\right.$

$\left\{\begin{array}{l}\text { When two Leaves } \\ \text { grow out of the fame } \\ \text { Point. }\end{array}\right.$

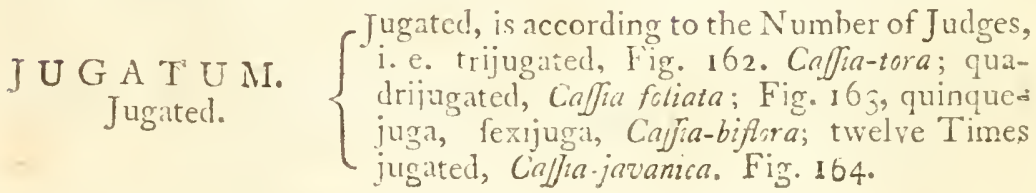




\section{$\left[3^{\mathrm{T}}\right]$}

\section{COMPOUND LEA VES.}

$\left.\begin{array}{l}\text { P E D A T U M. } \\ \text { Fig. 165. Arum dracunculus.. } \\ \text { Pedated. }\end{array}\right\} \begin{aligned} & \text { When a bifid Petiolus } \\ & \text { comnets Folioles on } \\ & \text { its interior Sides } \\ & \text { only. }\end{aligned}$ PINNATUM abrupinn. vel. ABRUPTUM-pin- $\int$ When a fimple Petiolus on its lateral Sides bears
atum.

Fig. 166.

Pinnated-abrupt, or Abruptly-pinnated.

PINNATUM cum impare.

Fig. 16\%:

Pinnated, terminating with a Foliole. many Folioles (without a zerminating Foliolum or Cii rhus.)

P I N N A T U M alternatum.

Fig. 168.
Pinnated-alternately.

$P \perp N$ N A T U M interruptum.

Fig. 169.

Pinnated abruptly.

PIN N A T U M

cirrhofum.

Fig. 170:

Pinnated with a Cirr-

hus.

$P ! N N A T U M$

articulatum

Fig. 17r.

Pinnated articulately.

P I N N A T U M decurfive.

Fig. 172.
Pinmated-decurfively.

$\mathrm{P} \perp \mathrm{N} N \mathrm{~A} \mathrm{~T} O$

pinnatum, vel.

Duplicato-pinnatum.

Doubly or twice pinnated.

PINNATO-triplicatum. vel triplicato-pmnatum.

Triple, or thrice pinnatica.
Fagara tragoides.

$\left\{\begin{array}{l}\text { Sorbus acuparia. } \\ \text { Quicken Tree }\end{array}\right\} \begin{aligned} & \text { Is a pinnated Leaf, ter- } \\ & \text { minating with a Fo- } \\ & \text { liole. }\end{aligned}$ $\left\{\begin{array}{l}\text { Amorpha Indigofera. } \\ \text { Indigo. }\end{array}\right\} \begin{aligned} & \text { Is a pinnated Leaf, } \\ & \text { with the Folioles al- } \\ & \text { ternate. }\end{aligned}$ $\left\{\begin{array}{l}\text { Agrimonia eupatoria. } \\ \text { Agrimony. }\end{array}\right\} \begin{aligned} & \text { Pinnated, with alter- } \\ & \text { nate imaller Folioles. }\end{aligned}$ $\{$ Pifum fotivim。 $\left\{\begin{array}{l}\text { Is a pinnated Leaf, ter- } \\ \text { minating in a Cirr- } \\ \text { hus. }\end{array}\right.$ A pinnated Leaf, whofe common Petiole is
articnlated. When the Bafe of ths $\left\{\begin{array}{l}\text { Melianthus major. } \\ \text { Honey Flower. }\end{array}\right\}$ Folioles are continued on the Sides of the Petiolus of a pin. nated Leaf. 


\section{[ 32$]$ \\ COMPOUND LEAVES.} CUIN A T U M. $\left\{\begin{array}{l}\text { Rubus fruticofus. } \\ \text { Bramble or Black-ber }-\end{array} \begin{array}{l}\text { Digitated, having five } \\ \text { Leaves }\end{array}\right.$ $\rightarrow$ Quinated $\quad$ ry.

SUPRA-decsmpofium. $\{$ Fumaria lutea. Fig. 1 75.
Supra-decompofite. $\left\{\begin{array}{l}\text { Yellow Fumatory. }\end{array}\right\} \begin{aligned} & \text { a many-times-divi- } \\ & \text { ded Petiolus- }\end{aligned}$ TERNATUMI-petiolatum.

Flatum.
Fig. $17^{5}$.
Ternated, with a Pet- $\left\{\begin{array}{l}\text { Cytifus-caja } \\ \text { Pigeon-pea. }\end{array}\right.$ iole.

TERNATUNI-Geffle. $\{$ Rhus lucidum.
Fig. 177.

Ternated-Seffile.

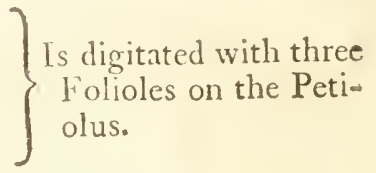

TERNATO-duplicatum, vel

D) uplicato-ternatum, vel

$$
\text { Fig. } 178 . \quad\{
$$

Biternatum.

Epimedium-alpinum. When three Folioles Three feffile Folioles。

Doubly or twice ternated.

Barren-wort. are on a Petiole, and each Petiole is ternas ted.

TRITERNATUM, vel

Triplicato-tenatum.

Fig. 179.

'Three Times ternated.

Or Triply ternated.

Aralia nudicaulis When a Petiole bears Berry-bearing Angeli- $\} \begin{aligned} & \text { three Folioles, and } \\ & \text { each of the Folioles }\end{aligned}$ Berry-bearing Angeli-
ca, with a naked
Stalk. 


\section{( 33 ) \\ $\begin{array}{llllllll}P & E & T & I & O & L & U & S .\end{array}$}

\section{Their FIGURE}

L I N E A R E . Fig. I. Linear.

A L A T US. Fig. 2. Winged.

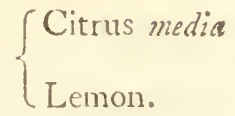

$\left\{\begin{array}{l}\text { Citrus aurantium } \\ \text { Orange. }\end{array}\right.$

C L A VA T US. Fig. 3 Clubb fhaped.
Every where the fame Breadth.

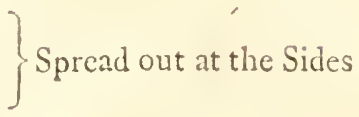

Thickened toward the Point.

ME
Figr. 4
Membranaceus. $\quad\left\{\begin{array}{l}\text { Anethum fonicu'um. } \\ \text { Fennel. }\end{array}\right\} \begin{aligned} & \text { Flat, Thin, and gene- } \\ & \text { rally pellucit. }\end{aligned}$ Membranaceus. Fennel.

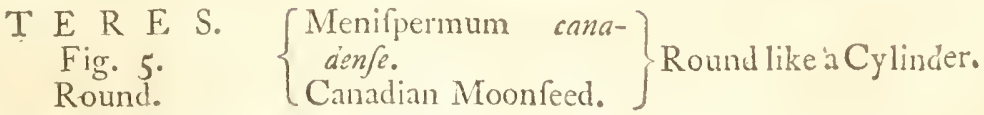

SEM I T E R E S.
Fig. 6.
Halfround. $\left\{\begin{array}{l}\text { Viola odorata } \\ \text { Violet }\end{array}\right\}$

T R I QUE T E R. Butomus luteus. Fig. 7 .

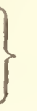

Three-cornered. Ruth

Flowering

CANALICUCATUS.
Fig. 8.
Canaliculated. $\quad\left\{\begin{array}{l}\text { Aconitum napelles. } \\ \text { Monkfhood }\end{array}\right.$

Channelled. 


\section{(34) \\ $\begin{array}{llllllll}P & E & T & I & O & L & U & S .\end{array}$}

\section{'Their F I G URE.}

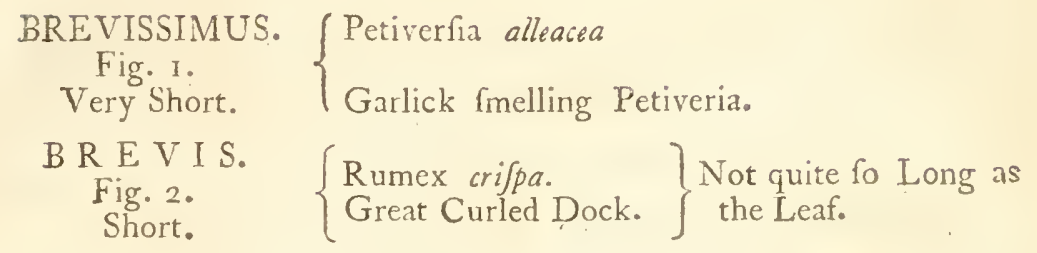

MEDIOCR IS.
Fig. 3.
Middling. $\left\{\begin{array}{l}\text { Humulus lepulus. } \\ \text { Hop. }\end{array}\right\} \begin{aligned} & \text { Of the Length of the } \\ & \text { Leaf. }\end{aligned}$

L. O N G U S.
Fig. 4.
Long. $\quad\left\{\begin{array}{l}\text { Potamogeton nataus. } \\ \text { Pondweed. }\end{array}\right\}$ Longer than the Leat.

LONGISSIMUS.
Fis. 5.
Very Long. $\quad\left\{\begin{array}{c}\text { Afafum canadenfe. } \\ \text { Arfarabeeca of } \\ \text { nada. }\end{array}\right.$ 


\section{$(35)$ \\ $\begin{array}{llllllll}P & E & T & I & O & L & U & S .\end{array}$}

\section{Their INSER T ION.}

A D N A T US.
Fig. I.
Adiule. $\quad\left\{\begin{array}{l}\text { Rheum palmatum. } \\ \text { Palmated Rhubarb. }\end{array}\right\} \begin{aligned} & \text { Inferted and adhering } \\ & \text { to the Stem. }\end{aligned}$

DECURRE N S.
Fig. 2.

Decurrent. $\quad$ Virginian Crotalaria. $\}$ Stem or Branch.

AIIPLEXICAULIS.
Fig. 3.
Amplexicaulo. $\left\{\begin{array}{l}\text { Saurus cernua. } \\ \text { Lizard's Tail. }\end{array}\right\} \begin{aligned} & \text { At the Infertion em- } \\ & \text { braces the Stem. }\end{aligned}$

APENTICULALIS.
Fig. 4.
Apenticuled. $\left\{\begin{array}{l}\text { Ononis cernua. } \\ \text { Reft-horrow. }\end{array}\right\} \begin{gathered}\text { A Leafy appendage ad. } \\ \text { hering to its Bafe. }\end{gathered}$

V A G I N A N S. f Canna Indica. T Having a Spatha, or Fig. 5. $\quad$ Indian flowering Reed $\}$ Embracing the Stem.
Vaginant. 


\section{$\left(3^{6}\right)$

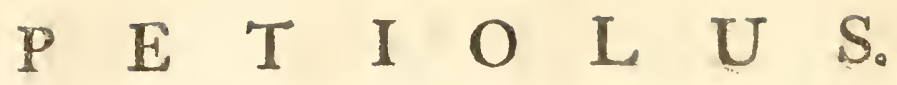

\section{Their DIRECTION.}

E R E C T U S.
Fig. I:
Erect. $\quad\left\{\begin{array}{l}\text { Chryfanthemum fero- } \\ \text { timum. } \\ \text { Creeping-rooted Chry- } \\ \text { fanthemum. }\end{array}\right\} U_{\text {pright. }}$

P A $\mathrm{T}$ E N N S.
Patent. $\quad\left\{\begin{array}{l}\text { Nerium oleander. } \\ \text { Rofe Bay. }\end{array}\right\}$ Spreading.

ASS UR GENS. \{ Sida radiata. TEending upwards in Affurgerit. $\quad$ Sida radiata.

RECURVATUS. $\left\{\begin{array}{l}\text { Paffiflora quadrangula- } \\ \text { ris. }\end{array}\right\}$ Fig. 4.
Recurvcd. $\quad\left\{\begin{array}{c}\text { ris. } \\ \text { Square-ftalk'd Paffion } \\ \text { Flower. }\end{array}\right\}$ Bent Backward. 


\section{(37) \\ $\begin{array}{llllllll}P & E & T & I & O & L & U & S .\end{array}$}

\section{Their S URFACE.}

G L A B E R.
Fig. I.
Smoothe. $\quad\left\{\begin{array}{l}\text { Menifpermum Cana- } \\ \text { denfe. } \\ \text { Canadian Moon-feed }\end{array}\right\}$ ACU L E A T U S. $\{$ Rubus fruticofus. Aculeated.

$N \underset{\text { Naked. }}{U} \mathrm{~T}$ S. $\{$ Fig. I having noleaves

ARTICUI,ATUS.
Fig. 3.
Artriculated. $\left\{\begin{array}{c}\text { Fagara pterota. } \\ \text { Lentifcus Leaved } \\ \text { Fagara. }\end{array}\right\}$ Jointed.

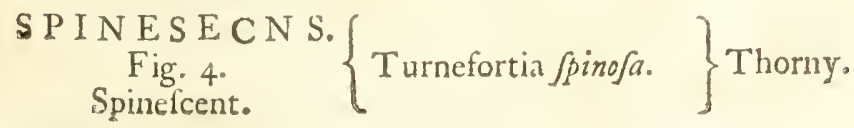




\section{$\left(3^{3}\right)$ \\ S T P U L $\mathrm{E}$.}

The Stipules are Appendages to the Leaf.

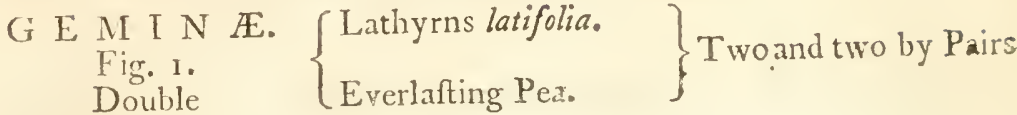

S O L I T A R I A
Fig. 2.
Single. $\left\{\begin{array}{l}\text { Melianthus major. } \\ \text { Honey Flower. }\end{array}\right\}$

LAT TERALES.
Fig 3.
Lateral. $\quad\left\{\begin{array}{l}\text { Paffiflora cerulca. } \\ \text { Com. Paffion Flowers. }\end{array}\right\}$ Marked in the Sides.

IXXTRAFOLIACEA. SFig. I. below the Bafe of the Petioles on the Without the Leaves. $l$ Outfide.

INTRAFOLIACEA. \{ Fig 2, on the infide, above the Bafe of the Within the Leaves. \{ Petioli

oppostTifoliA. Mercurialis perennis. $\{$ Placed on the Sides of Fig. 4.

Oppofite.

Dog's Mercury. $\quad$ the Leaves.

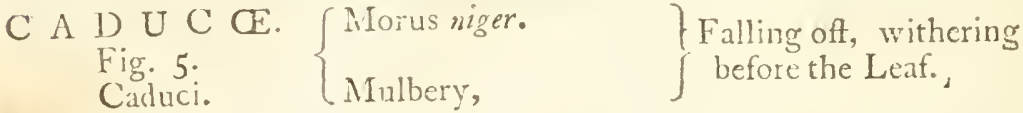

I) E C I D U Æ.
Fig. 6.
Deciduous. $\left\{\begin{array}{c}\text { Sorbus aucutaria. } \\ \text { Quicken Tree } \\ \text { Ilountain Af. }\end{array}\right.$ or $\{$ Faliing off Annually.

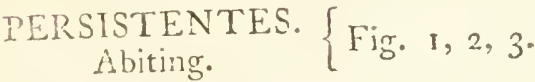

SPINESCENTES.
Fig. 7.
Spineticuent. $\quad\left\{\begin{array}{l}\text { Petiveria aleacea. } \\ \text { Garlick-finelling } \\ \text { tiveria. }\end{array}\right\}$

S E S S I L E S. $\{$ Fig. I, 2, 3, 4, 5, 6.

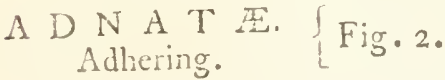




\section{$(39)$ \\ $S \quad T \quad I \quad P \quad U$ L A.}

DE CUR REN S.
Fig. I.
Decurrent. $\quad\left\{\begin{array}{l}\text { Rofa canina. } \\ \text { Dog Rofe. }\end{array} \quad\left\{\begin{array}{c}\text { Running down the } \\ \text { Branch. }\end{array}\right.\right.$

VAGINANTES.
Fig. 2. Vaginant. $\quad$ tia. SU B U L A T U M.
Fig. 3.
Subulate. $\left\{\begin{array}{l}\text { Petiveria Alliacea. } \\ \text { Garlick-linelling } \\ \text { beria. }\end{array}\right.$ Pe- $\left\{\begin{array}{l}\text { Shaped like an Aw } \\ \text { Awl-fraped. }\end{array}\right.$ Subulate.

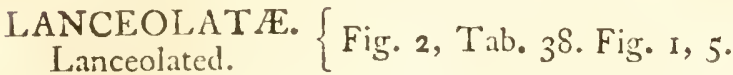

S A G I T T A T $\underset{\text { Sagittate. }}{ }\{$ Fig. I. Arrow-fhaped.

Lig. 4.
Lunculate. $\quad\left\{\begin{array}{l}\text { Humulus lupulus. } \\ \text { Hop. }\end{array}\right\}$ Noon-fhaped.

E R $\underset{\text { Erect. }}{\text { E }} \mathrm{C}$ Æ. $\{$ Fig. 3 .

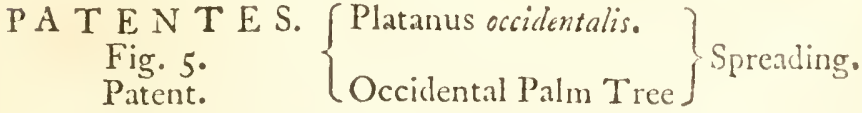

$\underset{\text { Intire }}{\text { INTETERRIGIME. }}\{$ Fig. 4, without divifion.

S E $\underset{\text { Serrated. }}{R} \mathrm{R}$ A T. $\{$ Fig. 5, Like a Saw.

C I I I A T $\underset{\text { Fig 6. }}{\text { Ciliated. }}\left\{\begin{array}{l}\text { Salvia horminum. } \\ \text { Red-topped Sage. }\end{array}\right\}$ Lafhed like Evelicis.

D E $\underset{\text { Dentated. }}{\mathrm{N} T \text { A }}$ 压. $\{$ Fig. 5, with Teeth.

F I S S Æ. \{Agrimonia Eufataria.

Split. Angrimony. 


\section{$\left(4^{\circ}\right)$}

\section{I R R H U S.}

\section{A TENDRIL or CLASPER.}

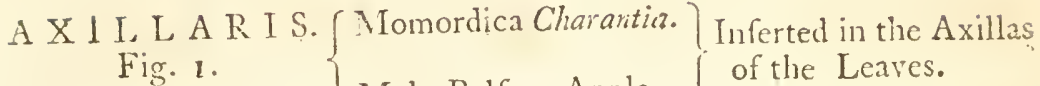
Axillary. Male Balfom Apple. $\int$ of the Leaves.

FO L I A R
Fig: 2. Foliar.

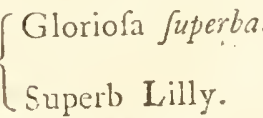

PETILORES. Fig. 3. Petiolar.

PEDUNCULARIS. Fig. 4. Peduncular.
Sitting on the Leaf.

Growing on the Foot$\int$ ftalk of the Leaf.

Growing on the Font. $\int$ Atalk of the Flower.

S I M P L E X. \{Fig. I, Undirided.

T R I $\underset{\text { Trifid. }}{\text { F }}$ D U S. $\{$ Fig. 3, Divided into Three.

MIUL T I F IDUS. $\{$ Fig. 4 , Divided into Many.
Multified.

CONvOLUTUS. f Fig. 4, Twifting in the fame Direction as the Convolute. $\quad\{$ Sun, in Rings.

REVOLUTUN.
Revolute. 


\section{[ $4 \mathrm{I}]$

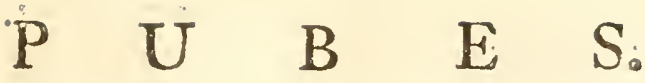

The $\mathrm{NAP}$.

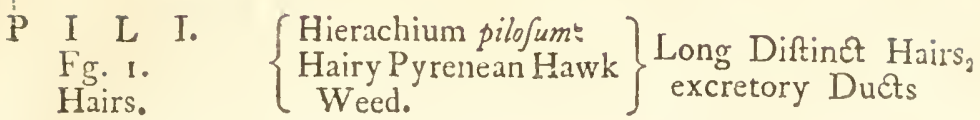

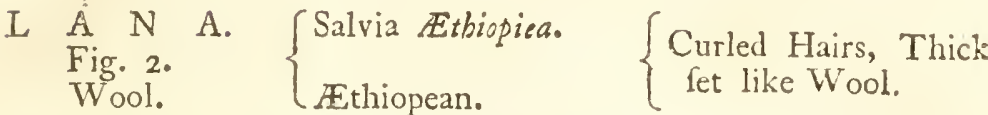

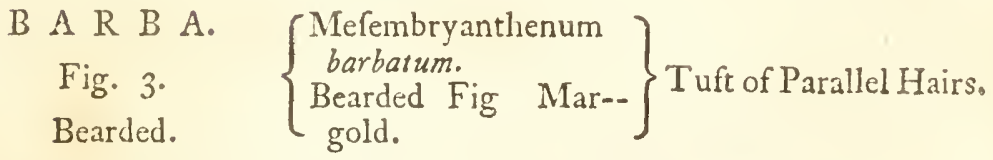

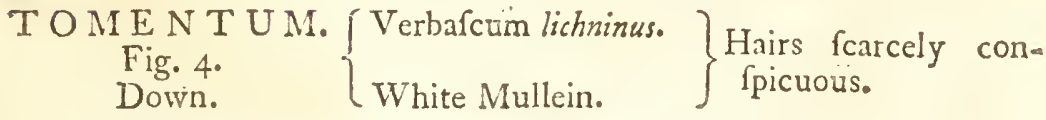
Down. Fig. 5.

GLOCHIDES. Fig. 6. Toothed. $\begin{cases}\text { Humulus petules. } & \begin{array}{l}\text { Prickles with the } \\ \text { Points bending down- } \\ \text { wards, having many } \\ \text { Teeth. }\end{array}\end{cases}$

$\mathrm{S}$
Fig.
Briftles. CE. $\quad\left\{\begin{array}{l}\text { Dipfacus fulonum. } \\ \text { Wildor manured Tea- } \\ \text { zel. }\end{array}\right\}$ Rigid Round Hairs.

S I M P L I C E S. j Cactus melocactus. Fig. 8. Simple. Melon Thiftle. Simple, not Divided。

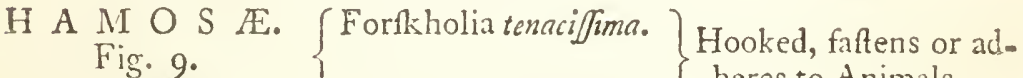
Hamous. LClammi Forkholea, $\}$ heres to Animals. 


\section{$(42)$ \\ B U B E S.}

RAMOSEv vel

FURCATA. Fig. I. Forked.

$\left\{\begin{array}{l}\text { Salvia Ethiopica. } \\ \text { Ethiopean Sage. }\end{array}\right\} \begin{gathered}\text { Subdivided into little } \\ \text { Branches. }\end{gathered}$

I L U MO US E. T T'crbafcum lichnite. Feathery, compofed Fig. 2. Plumous. [White Mullcin. $\quad\}$ Hair.

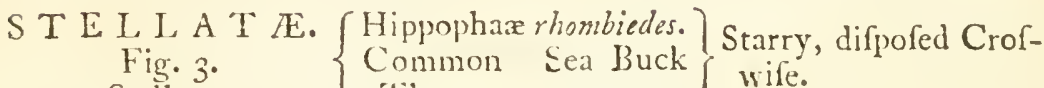
Stellate. Thorn.

HAAI RECURVATE

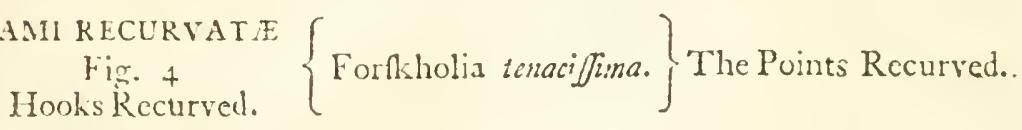

NANII INCURTAE. (Aretium lappa.

Iig. 5 .
Hooks Incurved. $\{$ Common Burdock. The Points Incurved.

G L O C H I DE S. (Humu'us lupulus. Glochid. Hop.

Prickles with many
Teeth, the Points
turned Back.

TRIGLOCHIT 2 . Triglochin palufire.
Fig. 7. Arrow-haped. WMant Triglochin. J Arrow- thaped.

Glandula. glands, little Teats for throwing out the Extremitous Humour of Plants, and are either feffil (/quat.); Stipilæt, having a Footltalk, or Powus; often perforating a Leaf. Vide, Fig 8 in Cheiranthus. Slock, Fuly Flower, Fig. 4.

UTRICULUS. (Sirracenin furpura. A little V'effel, replete Fig 9. \& 10. \{Purple Side Saddle with Secretory LiA little Veflil. fower. $\quad$ quor, as in Fig. 9, 10. (NEPENTLES. Dinillatoria.

I O I. I A E A. Annigdalus communis. A Are inferted on the Jo iccous. $\quad$ Bitter A!non Tr:e, $\}$ Leares, and Fig. 10. 


\section{(43) \\ B U B E S.}

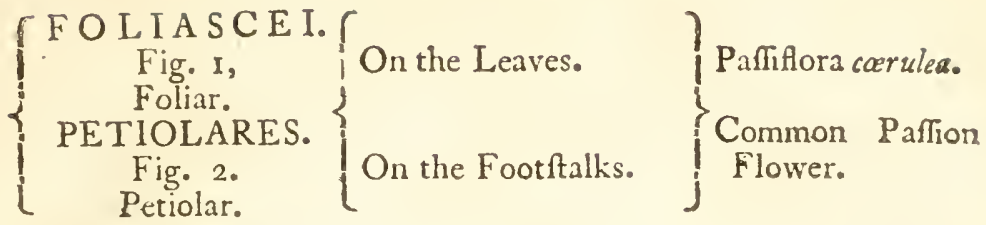

S T I P UL A R E S.
Fig. 4.
Stipular. $\left\{\begin{array}{c}\text { Bawhinia divaricata. } \\ \text { Dwarf Mountain E- } \\ \text { bony. }\end{array}\right\}$ Inferted in the Stipuls. VISC OS I T A S. $\{$ Cucubalus vifiofa.
Fig. 5 . Vifcous. LClammy Campion. $\}$ my Quality.

GLUTINOSUS. \{ Salvia glutinofa. $\}$ A humour whofe qua$\begin{array}{l}\text { Fig. } 6 . \\ \text { Glutinous. }\end{array}$ Yellow Sage, or Clary $\} \begin{aligned} & \text { lity is of a lubricat- } \\ & \text { ing Slippery Nature. }\end{aligned}$ 


\section{(44) \\ A $\mathrm{R} \quad \mathrm{M}$ A。 \\ G U $\quad A \quad R \quad B \quad S$.}

Acculei, harp Prickles fixed on the Bark of Plants:

R E C T I.

Fig. I.

Res.

\{ Solanum mamofum. $\left\{\begin{array}{l}\text { Straight, without } \\ \text { bending. }\end{array}\right.$

I C U R V I.
Fig. 2.
Incurved. $\left\{\begin{array}{l}\text { Rubus frutiofus. } \\ \text { Bramble. }\end{array}\right\}$ Bending inwards.

R E C U R V I.
Fig. 2.
Recurved. $\left\{\begin{array}{c}\text { Zanthoxilum-clava } \\ \text { hercules. } \\ \text { Tooth-ach Tree. }\end{array}\right\}$ Bending outwards.

F U R C $Æ . \quad\{$ Ribes grofularia.
Fig. 4. Prickles, divided into Forked. $\quad$ Common Goofebery. $\}$ many Forks.

B I F I D E. $\{$ Fig. 4 Divided into two.

T R I F I I D $Æ$ Trifid. $\{$ Fig. 6, Divided into Three. 


\section{(45) \\ A $\mathrm{R} \quad \mathrm{M}$ A.}

Spina a Spine Gands - Tharp Prickle fixed in the Wood of the Trunk or Branch.

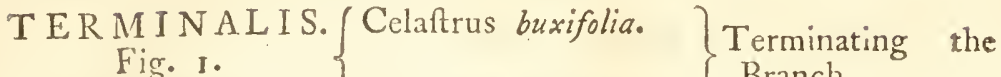
$\begin{array}{l}\text { Fig. I. } \\ \text { Terminal. }\end{array}$ Box-leav'd Staff Tree. $\}$ Branch.

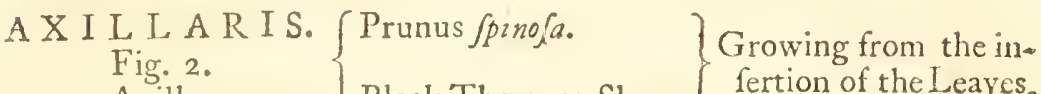
Axillar. Black Thorn or Sloe. $\}$ fertion of the Leaves.

C A L I C I N E.
Fig. 3.
Calixine. $\left\{\begin{array}{l}\text { Carduus nutans. } \\ \text { Mufk Thiftle. }\end{array}\right\} \begin{aligned} & \text { Growing on the Cup } \\ & \text { or Calax. }\end{aligned}$ F O L I A R I S.
Fig. 5.
Foliar. $\quad\left\{\begin{array}{l}\text { Yucea gloriofa. } \\ \text { Supert, Adam's Nee- } \\ \text { dle. }\end{array}\right\}$ Growing on the Leaf. S I M P L E X. $\{$ Fig. I, 2, 3, 5. Undivided Single.

5 On the Pericarpum or Fruit Datura. Stramonium.
Thorn Apple. D I V I S A. $\quad\{$ Into Two. $\quad\} \begin{aligned} & \text { Artuina by/pino/a. } \\ & \text { Fig. } 6 . \\ & \text { Divided. }\end{aligned}$ Two-fpined Artudina.

Fig. $\%$
Divided into Several,
or
Severally Divided. $\left\{\begin{array}{l}\text { Gletitzia-tricanthus. } \\ \text {. }\end{array}\right.$

S T I M U L I. Fig. 8. Stings. $\left\{\begin{array}{l}\text { Urtica dioica. } \\ \text { Common Nettle. }\end{array}\right\} \begin{aligned} & \text { The Stingsmaking in- } \\ & \text { flamatory punoturcs, } \\ & \text { which go off with an } \\ & \text { itching. }\end{aligned}$ 


\section{[ 46 ]}

\section{B R A C T E.}

Are F L ORA L L E A V ES.

$\left.\begin{array}{c}\text { CO L O R A T CE. ; Salvia horminum. } \\ \text { Fig. I. } \\ \text { Coloured. }\end{array}\right\}$

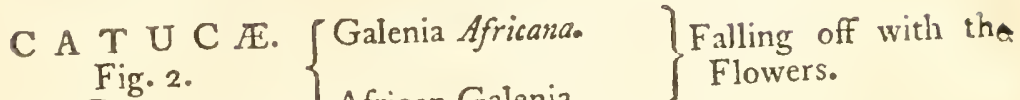

LAfrican Galenia. DE C I D U Æ.
Fig. 3.
Defiduous. $\left\{\begin{array}{l}\text { Phytolacca decandria. } \\ \text { Virginian Poke. }\end{array}\right\}$ Falling off.

PERSIS T
Fig. 4 . Perfifting.

C O M A. $\left\{\begin{array}{l}\text { Fritellaria-corona } 1 \mathrm{~m}^{-} \\ \text {periales. }\end{array}\right.$ Terminating in leaves

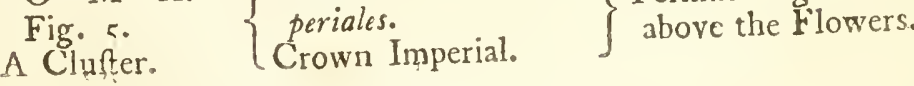




\section{(47) \\ P E D U N G L U S:}

The Footftalk of Flowers.

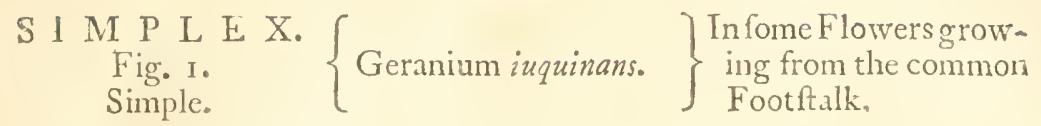

$\underset{\text { Fig. 2. }}{\text { C O M U E. }}\left\{\begin{array}{l}\text { Anæthum foenicalum. } \\ \text { A Footftalk common } \\ \text { to many Flowers. }\end{array}\right.$ Common.

P R O P R I UM.
Proper. Fig. I, 2, $\left(a_{2}\right)$

P A R T I A L IS.

Fig, 3.

Fig. 2, 3, (b.)

P E T I C E L L U S. $\{$ Fig. 1, 3. (a.)
A Little Footitalk. 


\section{$(48)$ \\ PEDUNCULUS.}

\section{Their PLACE.}

$\left.\begin{array}{c}\text { S C A P U S. }\left\{\begin{array}{l}\text { Stratiotes alaides. } \\ \text { Water Aloe, or } \\ \text { Water Soldier. }\end{array}\right. \\ \text { Fig. 1. } \\ \text { Stalk. }\end{array}\right\} \begin{aligned} & \text { A Pediolus Rifing from } \\ & \text { the Root refembling } \\ & \text { a Stalk. }\end{aligned}$ $\underset{\text { Railical. }}{\text { R A D I A }}\{$ Fig. I.

C A U L I N U S. $\left\{\begin{array}{l}\text { Convallaria multiflora. } \\ \text { Broad-leav'd Solo- } \\ \text { Fign's Seal. }\end{array}\right\}$ Springing from the
Caline.

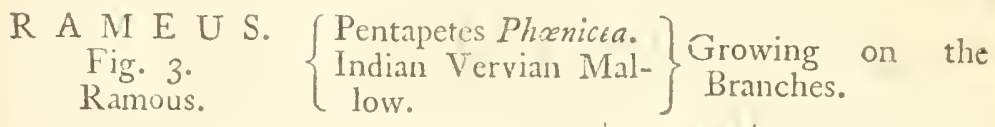

1'ET I O L A RES. [Tumera Ulmifolia. T Growing on the PetiFig. 4. Petiolare. the Leaf. 


\section{(49) \\ P E D U N C U L US.}

\section{Their PLACE.}

CIRRHIFEROUS.
Fig. I.
Cirrhiferous. $\left\{\begin{array}{l}\text { Viis viniferä. } \\ \text { Vine. }\end{array}\right.$

Growing from the Tendrill, or Clafper.

TER MINALIS.
Fig. 2.
Terminal. $\left\{\begin{array}{l}\text { Coronilla Valentina. } \\ \text { Small Shrubi Coronilla }\end{array}\right\} \begin{aligned} & \text { Terminating } \\ & \text { Branch. }\end{aligned}$ the

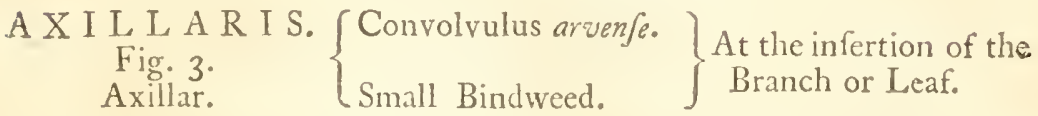
LATERIFLORA.
$\begin{gathered}\text { Fig. 4. } \\ \text { Lateritiorus. }\end{gathered}$$\left\{\begin{array}{l}\text { Afclepias vincitoxeum. } \\ \text { Yellow officinal Swal- } \\ \text { low-wort. }\end{array}\right\} \begin{aligned} & \text { On the Sides of the } \\ & \text { Leaves. }\end{aligned}$

OPPOSITIFOLIA. Symphitum officinalis.

Fig. 5.

Fig. 5. $\left\{\begin{array}{l}\text { Common Comfrey. }\end{array}\right.$ Having oppofite leaves

INTRAFOLIACFA. $\{$ Rufcus Aculeatus.
Fig.6.

Within the Leaves. LButcher's Broom.

Growing on the infide of the Leaf. 


\section{[ 50 ] \\ PEDUNCULUS. \\ Their S I TU A T IO N.}

A L T E R N I.
Fig. I.
Alternate. $\quad\left\{\begin{array}{l}\text { Clutia pulcbella. } \\ \text { Broad-leav'd Clutia. }\end{array}\right.$

S P A R S I.
Fig. 9.
Sparfed.! $\quad\left\{\begin{array}{l}\text { Celtis aufrails. } \\ \text { European Nettle Tree }\end{array}\right\} \begin{aligned} & \text { Scattered, irregularly } \\ & \text { placed, }\end{aligned}$

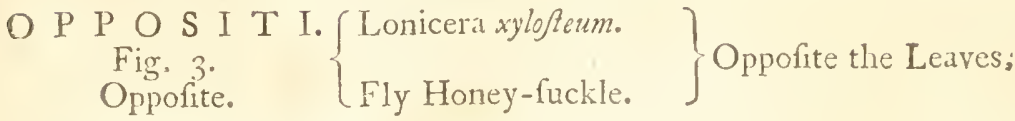

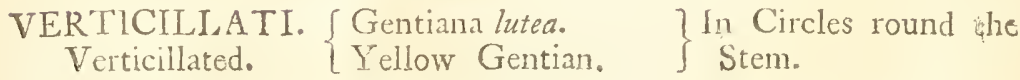




\section{[ 5I ] \\ PEDUNGULUS.}

Their NUMBER。

SOLITARIUS. Fig. I. Solitary.

GE M IN A T U S. P Pentapetes Phenicea.

Fig. 2. $\quad\left\{\begin{array}{c}\text { Indian Vervian Mal- } \\ \text { low. }\end{array}\right.$ By Two, or in Pairs.

UMBELI, UL R. CComus fanguineus. ? Having many PedunFig. 3. $\{$ Cos from the fame Little Umbel. LCommon Dogwood. Centre. 


\section{[ 52 ] \\ PED U N C U L US.}

\section{Their D I R E C T ION.}

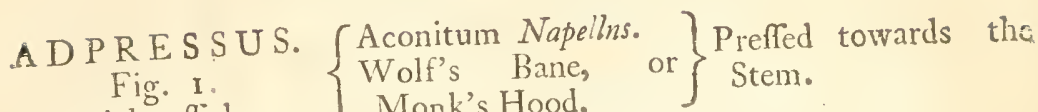
Adprefled.

E R E C T U S. $\left\{\begin{array}{l}\text { Paris quadrifolia. } \\ \text { Herb Prris, or True } \\ \text { Erect. }\end{array}\right\}$ Upright.

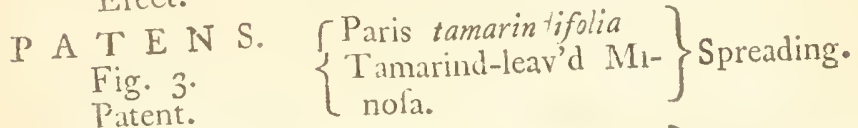

CONFERT US.
Fig. 4.
Confert. $\left\{\begin{array}{l}\text { Satureja Fuliana. } \\ \text { Linear-leav'd Savory. }\end{array}\right\}$ Clofe together. C E R N U U S. $\left\{\begin{array}{l}\text { Trillium cernuus. } \\ \text { Fig 5. } 5 \text {. } \\ \text { Drooping. }\end{array}\right.$ Flower'd Tril- $\}$ The Point looking
lium.

RESUPINATUS.
Refupinate. Fig. 6.

Declining. Apple. wife.

$\underset{\text { Notting. }}{\mathrm{N}} \underset{\mathrm{T}}{\mathrm{A}} \mathrm{N} . \quad\{$ Fig. $5,6$. FL A C C I D U S.
Flaccid. Fig. 6.

Slender, weak, the weight of the Flower

$\int$ makes it hang down- 


\section{(53) \\ PE D U N C U L U S.}

\section{Their DIRECTION.}

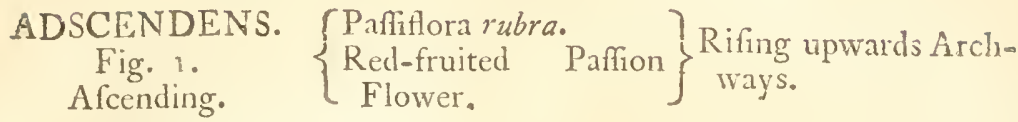

P E N D U L U S. \{Cytifius laburnam. Fig. 2. $\quad$ Common Laburnam. $\}$ Hanging lofe.

S T R I C T U S. (Xeranthemumannum. Fig. 3. Strict. $\left\{\begin{array}{l}\text { Eternal or Satin Flow- } \\ \text { er. }\end{array}\right\}$ Streight, Stiff。

FLEXUOSUS. (Tillandia tenucifolia.

Fig. 4 .

Flexed.

$\{$ Narrow-leav'd Tillandfia.

RETROFRACTUS

Retrofrast. 


\section{( 54$)$ \\ $P E D U N C U L U S 。$}

\section{Their STR UCT URE.}

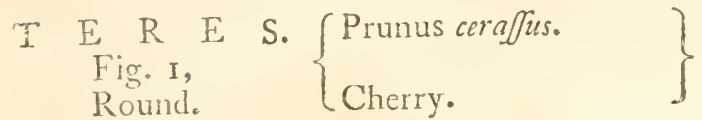

T R I QUE T E R.
Fig. 2.
Three Sided. $\{$ Heleborus fativa.

TETRAGONUS.
Fig. 4.
Four-Sided. $\quad\left\{\begin{array}{l}\text { Parnoflia paluftis. } \\ \text { Grafs of Parnotus. }\end{array}\right.$

$\left.\begin{array}{l}\underset{\text { Fig. } 4 .}{\text { Filiform. }} \\ \text { Yellow Velching. }\end{array}\right\}$ Thread Shaped.

ATTENUATUS.
fig. 5
Aitenuated. $\quad\left\{\begin{array}{l}\text { Rhododendron ponti- } \\ \text { cum. } \\ \text { Purple Rhododerdron. }\end{array}\right\} \begin{aligned} & \text { Tapering upwards } \\ & \text { contrary to Clavatus. }\end{aligned}$

$\left.\begin{array}{c}\text { C L A V A T U S. } \\ \text { Fig. 6. Helianthus anna. } \\ \text { Clubb-hajed. }\end{array}\right\}$

INCR ASATUS.
Fig. 7
Incrate. $\quad\left\{\begin{array}{l}\text { Hamamelis virginir. } \\ \text { Witch Hazel. }\end{array}\right\}$ Swclling Upwards. 


\section{(55) \\ PEDUNCULUS.}

Their S T R U C T U R E:

N U D U S.
Fig. I.
Naked. $\quad\left\{\begin{array}{l}\text { Napcea lavis. } \\ \text { Sinuth Napæa. }\end{array}\right\}$

S QU A M O S U S.
Fig. 2.
Squamus. $\quad\left\{\begin{array}{l}\text { After hy Jopifolia. } \\ \text { Hyffop-leav'd } \\ \text { wort. }\end{array}\right.$ Star- $\}$ Scaly.

F O L I A T U S.
Fig. 3.
Foliatet. $\quad\left\{\begin{array}{l}\text { Chironia frutefieus. } \\ \text { Shrubby Chironia. }\end{array}\right\}$ Leafy. BRA CTEA TUS.
Fig. 4.
Bracteated. $\quad\left\{\begin{array}{l}\text { Tilia Europaa. } \\ \text { Common Lime Tree. }\end{array}\right\} \begin{aligned} & \text { Furnifhed with a Flo- } \\ & \text { ral Leafo. }\end{aligned}$

GENICULATUS,
Fig. 5.
Geniculated. $\left\{\begin{array}{l}\text { Hibifcus Zeylancia. } \\ \text { Ceylanian Hibifcus. }\end{array}\right\}$ Jointed.

Articulated. 


\section{$\left(5^{5}\right)$ \\ PED UNCULUS.}

\section{Their S I Z E.}

BREVISSIMUS.
Fig. 1.
Very Short. $\quad\left\{\begin{array}{l}\text { Citrus aurantium. } \\ \text { Orange. }\end{array}\right.$

B R $\underset{\substack{\text { Fig. } \\ \text { Short. }}}{\text { Eh I S. }} \quad\left\{\begin{array}{l}\text { Rumex crijpa. } \\ \text { Curled Dock. }\end{array}\right.$

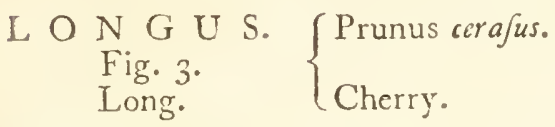

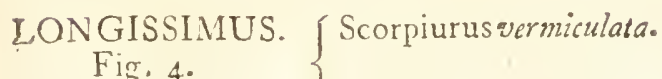
Very Long. $\quad$ Common Caterpillar. 


\section{[ 57 ] $]$ \\ I NFLORESCE N S,}

Is the Manner by which Flowers are joined to the Plant by the Peduncle or Footftalk.

T E R MI I N A L IS. \{ Coronilla valentina.

Fig. I.

Terminal. S Small Shrubby Coronilla.

L A T ER A L IS.

Fig. 2.

Lateral.

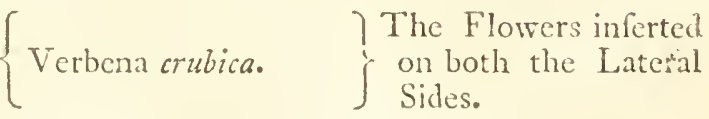

SEMILATERALIS. $\{$ Nardus Seriza.

Fig. 3.

Semilaterai.

Mtat Grafs.

The Flowers inferted $\int$ on one fide only.

S E C U N D US. \{ Fumaria lutea.

Fig. 4 .
Inclining to one Side. $\{$ Yellow Fumatory.

S P A R S I S.
Fig. 5.
Sparfed. $\quad\left\{\begin{array}{l}\text { Amightalus perfica. } \\ \text { Peach Tree. }\end{array}\right\}$ Irregular difperfed. S E S S I L E S. $\left\{\begin{array}{l}\text { Daphne mezerium. } \\ \text { Fig. 6. } \\ \text { Seffile. }\end{array}\right\} \begin{aligned} & \text { Setting clofe without } \\ & \text { Footitalk. }\end{aligned}$ Wezerium.

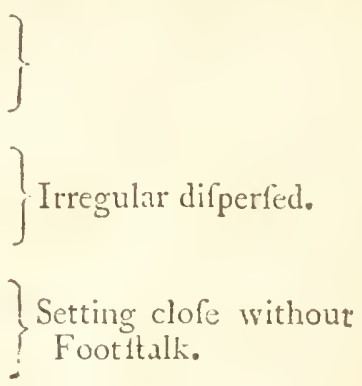

PEDUNCULATUS.
F̈ig. $7 \cdot$
Peduncled. $\left\{\begin{array}{l}\text { Jafminum offinalis. } \\ \text { Jafmin. }\end{array}\right\}$ Having Footftalks.

SO L I T A R I US. $\left\{\begin{array}{l}\text { Xeranthemum anumm } \\ \text { Fig. 8. } \\ \text { Solitary. }\end{array} \quad\left\{\begin{array}{l}\text { Bearing only one } \\ \text { er. }\end{array}\right.\right.$ 


\section{$\left(5^{8}\right)$ \\ I N F L ORESCENS.}

UNI F L OR U S.
Fig. I.
One Flower. $\left\{\begin{array}{c}\text { Geranium fanguinesm. } \\ \text { Lancafhire Crane's } \\ \text { bill. }\end{array}\right\} \begin{gathered}\text { Many Flowers on } \\ \text { one Footftalk. }\end{gathered}$ $\begin{array}{l}\text { B I F L O R U S. } \\ \text { Fig. 2. } \\ \text { Two Flowers. }\end{array} \quad\{$ Geranium robertianum. $\left.\}\right\} \begin{gathered}\text { Bearing Two Flowers } \\ \text { on the Footftalk. }\end{gathered}$

TR I FL OR US.
Fig. 3.
Three Flowers. $\quad\left\{\begin{array}{cc}\text { Volkamaria inermis. } \\ \text { Long-leav'd } \\ \text { Volkanaria. }\end{array}\right.$

MIULTIFLORUS. \{ Geranium mofchatel- T Many Flowers on one Fig. $4 . \quad\left\{\begin{array}{l}\text { Getina. } \\ \text { lina }\end{array}\right.$ Footftalk. Many Elowers. 


\section{( 59$)$ \\ I NFL O R E S E N S。}

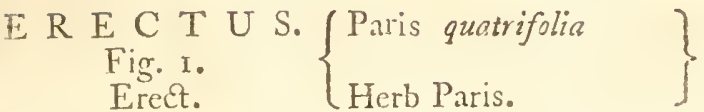

C E R N U U S.
Fig. 2.
Drooping. $\{$ Trillium cernuzm.

$\mathrm{N} \mathrm{U} \mathrm{T} A \mathrm{~N} S$.
Fig. 3.
Nodding. $\quad\left\{\begin{array}{l}\text { Carduus nutan: } \\ \text { Muks Thifle. }\end{array}\right\}$

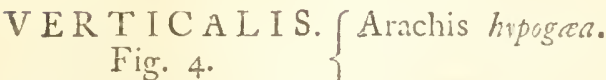

Vertical. American Earth Nut.

HORIZONTALE
Fig. 9.
Horizontal. $\quad\{$ Elathericum Carthagenenfis: 


\section{$\left[\begin{array}{lll}60 & \end{array}\right]$ \\ I N F L O R E S C E N S.}

VERTICILLUS. \{ Many Flowers growing round the Stalk in Whorled. $\quad$ Circle.

S E S S I L E S.
Fig. 1.
Seffill. $\quad\left\{\begin{array}{l}\text { Salvia glutinofa. } \\ \text { Yellow Sage or Clary. }\end{array}\right\} \begin{aligned} & \text { Squat, without any } \\ & \text { manifeft Footltalk. }\end{aligned}$ $\left.\begin{array}{c}\begin{array}{c}\text { PEDUNCULATUS. } \\ \text { Fig. 2. } \\ \text { Pedunculated. }\end{array} \\ \text { Black Horehound. }\end{array}\right\} \begin{aligned} & \text { Ballota nigra. } \\ & \text { the Flowers. }\end{aligned}$

$N \underset{\text { Naked. }}{\mathrm{T}} \mathrm{U}$ S. $\quad\{$ Fig. I, having no Involurum.

INVOLUCRATUS. \{ Galeobtelon lutenm. \}Furnifhed with an In $\begin{array}{l}\text { Fig. 3. } \\ \text { volucrated. }\end{array}$ Yellow Galeobtelon. $\}$ volucrum.

BRACTEATUS.
Fig. $4 \cdot$
Bracteated. \{

CON FER T US.
Confert. Fig. 12, 4. Clofe together.

D I S T A N S. $\{$ Fig. 3, diftant from one another. 


\section{(6r) \\ I N F L O R E S E N S.}

Capitalum, when many Flowers together forming a Globe.

SUBROTUNDUM. $\{$ Trifolium pratenfe.
Fig. . . Subrotund. $\quad$ Purple Trefoil or Glover.

GL O B O S U M. \{ Echinops pharacaphlus. Fig. 2. $\quad$ Globe Thiftle.

DIMIDIATUM.
Fig. 3 Trifolium repens. Fig. 3.
Halfround.

F O L I O S U M.
Fig. 4.
With Leaves. $\quad\left\{\begin{array}{l}\text { Trifolium glomer atum. } \\ \text { Round-headed Trefoil. }\end{array}\right.$

N UD U M.
Fig. 5
Nol. $\quad\left\{\begin{array}{l}\text { Trifolium alpium. } \\ \text { Alpine Trefoil. }\end{array}\right.$

FASICULATUM. \{ Dianthus barbatum.

Fafficled. ISweet William. 


\section{$(62)$}

\section{N F L O R E S C E N S.}

Spica, a Spike, the Flowers are Seffile growing round alternate on a common Peduncle.

S I M P L E X.
Fig. 1.
Simple. $\{$ Cyperus monofachium. $\}$ A fingle Spike undiviCOM POS I T A.
Fig. 2.

Compofite. Bonus Henricus. growing from the common Peduncle.

GLOMERATUS. Scirppus holoschoetus \} Mariy little Spikes or

Fig 3.
Glomerate. $\quad\left\{\begin{array}{l}\text { Round-headed Club- } \\ \text { rufh. }\end{array}\right\} \begin{gathered}\text { Globules } \\ \text { together. }\end{gathered}$

O V $\underset{\text { Fig. 4. }}{\text { A }}$ T A.
Ovate. $\left\{\begin{array}{l}\text { Lagurus ovaius. } \\ \text { Oval Spiked Lagurus. }\end{array}\right\}$ Egg thaped.

VENTRICOSA.
Fig. 5,
Ventrious. $\quad\left\{\begin{array}{l}\text { Phalaris arundiana. } \\ \text { Red Canary Grafs. }\end{array}\right\}$ Swoln, goutya

CY LIN DR ICA.
Fig. 6.
Cylidrical. $\quad\left\{\begin{array}{c}\text { Phleum pratenfe. } \\ \text { Mleadow Catt's-tail } \\ \text { Grafs. }\end{array}\right\}$ 


\section{( 63 ) \\ $S \quad P I C A$.}

3 E C U N D A.
Fig. I.
One Sided. $\left\{\begin{array}{l}\text { Nardus Stricia. } \\ \text { Mat Grafs. }\end{array}\right\}$

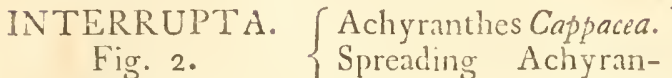

Fig. 2.
nterrupt. $\quad \begin{gathered}\text { Spreading Achyran- } \\ \text { thes. }\end{gathered}$ Alternately Smaller. I M B R I C A T A. $\left\{\begin{array}{l}\text { Salvia Hifpanica. } \\ \text { Fig. 3. } \\ \text { Spricated. }\end{array}\right\}$ Placed like Scales or
Tiles on a Houfe.

ARTICULATA.
Fig. $4 \cdot$
Articulated. $\quad\left\{\begin{array}{l}\text { Tripfacum dacillis. } \\ \text { Jointed Tripfacum. }\end{array}\right\}$

A A O S A.
Fig. 5.
Ratuous. $\quad\left\{\begin{array}{l}\text { Eriophorumpolifachis. } \\ \text { Cotton grafs. }\end{array}\right\}$ Branching Varioufly。

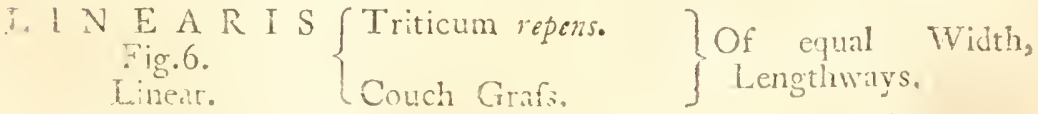




\section{( 64 )

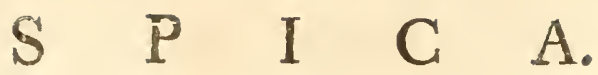

A Spike, the Flower growing on a common Peduncle,

C I L I A T A. (Phleum pratenfe.

Fig. I. ¿Cat's Tail Grafs.

F O L E A C E O.
Fig. 2.
Leafy. $\{$ Verbena orbica.

C O M O S A. \{Lavandula Stachas.

Tufted or Crowned. \{ French Lavender. 


\section{$\left[\begin{array}{lll}6 & \end{array}\right]$ \\ C O R Y M B U S.}

A kind of Spike, whofe Flowers are furnifhed with Footfalks fo proportioned to their fituation as to elevate all the Flowers of the Spike to the fame height

S I M P L E X. \{ Spirea opulifolia.

Fig. I.

Simple. $\quad$ Virginian Gilder Rofe, or Spirea.

COMPOSITA. S Senecio Facobea.

Fig. 2.
Compofite. Common Ragwort. 


$$
\begin{array}{cccccc}
(66) \\
T & H & \text { I } & \text { S } &
\end{array}
$$

A kind of a crowded Panicle of an Ovate Form.

D I F $\underset{\text { Fig }}{\mathrm{F}}$ U S U S. . Syringa vulgaris.

Diffule.

Lelac.

F O 1. I A L U S. $\int$ Tuffilago alba.

Fig. 2

Foliated. White Colt's Foot. 


\section{$\left[\begin{array}{ll}6 & \end{array}\right]$ \\ RACEMUS.}

A Bunch of Flowers, the Peduncles coming at the Sices. S I M P L E X. $\left.\begin{array}{l}\text { Phytolacca decandria. } \\ \text { Fig. I. }\end{array}\right\}$ Simpic. American Nighthade. Undivided. COMPOSITA. Vitis vinifera. Fig. 2. Compolite. LVire.

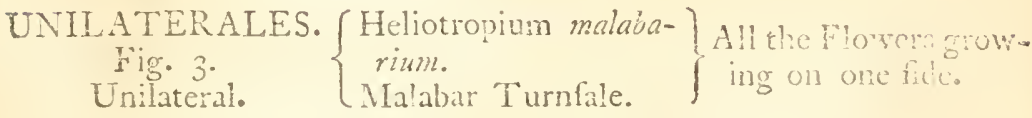

SECUNDU S. \{ Lathyrus latifulia. T The Flower Dendiag

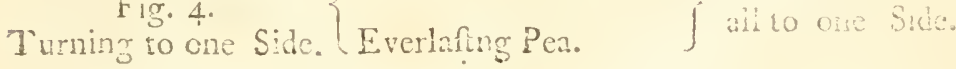




\section{(68)}

\section{R A C E M U S.}

PE D A T U S.
Fig. I,
Pedate. $\left\{\begin{array}{l}\text { Limodorum finuatum. }\} \\ \begin{array}{l}\text { The Foot ftalk coming } \\ \text { on one fide, like the } \\ \text { Toes of the Feet. }\end{array}\end{array}\right.$ CONJUGATUS.
Conjugated. Fig. I joined by two.

E R E C T U S.
Fig. 2.
Erect.

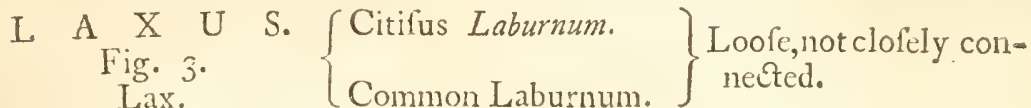
N $\begin{gathered}\text { U } D \text { D U S. } \\ \text { Nod. } \\ \text { Nodding. }\end{gathered} \quad\left\{\begin{array}{l}\text { Ribes rubra. } \\ \text { Red Curraits. }\end{array}\right\}$ Hanging downwards. F O L ] A T US.
Fig. 5.
Folinted. $\quad\left\{\begin{array}{l}\text { Arbutus unedo. } \\ \text { Strawberry Tree. }\end{array}\right\}$ Having Leaves. 


\section{(69) \\ P A N I G U L A.}

A Panicle, the Flowers fcattered on Peduncles that are divided in different Forms.

D I F F U S \&.
Fig. I.
Diffule. $\left\{\begin{array}{l}\text { Avena fatua. } \\ \text { Wild Oat. }\end{array}\right.$ COMPOS I T A. 2.
Compofite. $\quad\left\{\begin{array}{l}\text { Bromus Mollis. } \\ \text { Field Broom Grafs. }\end{array}\right\} \begin{aligned} & \text { When manv Flowers } \\ & \text { come together. }\end{aligned}$

S I M $\underset{\text { Simplit. }}{\mathrm{P} L \mathrm{E}}$ X. $\{$ When but few Flowers, 


\section{$\left\lfloor\begin{array}{lll}10 & 1\end{array}\right.$ \\ IRUCTIFICATIO.}

Fruetification, a temporary part of Vegetables called the Generation.

Calyx, a Flower Cup, is the Termination of the outer Bark of the Plant, prefent in the Fruatification.

Periantheum, a Flower Cup, whofe Station is clofe to the Fructification.

FRUCTIEICATIONIS. (Rubus fruticofus.

Fig. 1.

Of the Fructication. Common Bramble. FLOR $1 \mathrm{~S}$.

Fig. 2.

Of the Flower.

F R U C T U S.

Fig. 3.

Of the Fruit.

PROPRIÜ II

Fig. 4.

Proper.

MONOPRYLEUN (Citrus aurantium.

Iix 5.

Nenobhyllous.

POETHELLUM.

Fir. 6.

Polyphllous.

B I F I D U II.

Fig. 7 .

Bifid.

TRIFIDUII.

Fig. 8 .

Trifid.

OUADRIFLDUN.

Fig. 9.

Quadrifit.

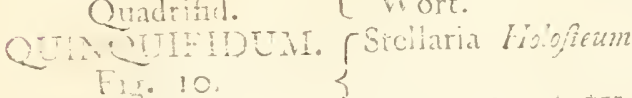

Orange.

Mercurialis perennis.

Dog's Miercury.

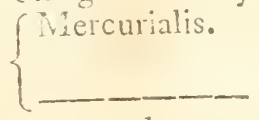

CEnanthe cracata.

Hemlock.

Geropogon giabrum.

L Did Mian's Beard .

$\{$ Tumaria intca.

I ellow Fumatory.

$\{$ Rumax crifpa.

Great currled Dock.

Sagna p. scumbent.

Procumbent Pearl

Wort.

Fis. 10.

Qunguifu.
When containing botl.

Stamens and Germen

Containg the Stamina without the Germen: Containing the Germen without the Stamina.

With refpect to the $\int$ Flower.

Confifing of one leaf. Confifing of many Leaves.

Dividad into $\mathrm{T} w 0$.

Divided into Three.

$\int$ Dirided into Four. 


\section{( 70$)$ \\ C A L Y X.}

MIULTIFIDUM.
Fig. I I.
Multified. $\quad\left\{\begin{array}{l}\text { Ceratophyllum demer- } \\ \text { fum. } \\ \text { Prickly feeded Hom- } \\ \text { wort. }\end{array}\right\}$ Many times Divided.

BIPARTITUM. \{Adoxa Tuberofa. \Divided into Two

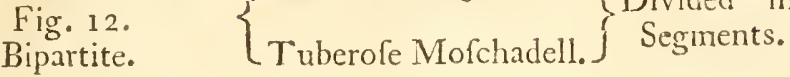
TRIPARTITUM. $\{$ Mercurialis perennis. $\}$ Divided into Three
Fig. 1 3.

Tripartite.

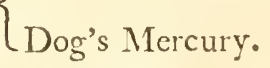

Segments.

QUADRIPARTITUM $\{$ Morus niger.
Fig. 14.

Cuadripartite. Mulberry.

Dividéd into Four $\int$ Segments.

QUINQUEPARTITUM. Parnaflia paluftris.

Fig. I 5.
Quinquepartite. Grafs of Parnaffus.

\}Into Five Segments.

I N T E G G U M.I $\{$ Hura crepitons.

Integer, or Intire. $\quad$ Sand-box Tree.

\} Undivided.

$\underset{\substack{\text { Fig. I } 7 \\ \text { Tubulous. }}}{\text { TuBU. }}\left\{\begin{array}{l}\text { Monarda Fiftulofa. } \\ \text { Fiftulous Monarda. }\end{array}\right\}$ Forming a Tube.

P A T E N S.
Fig. i 8.
Patent. $\quad\left\{\begin{array}{l}\text { Paris Ruadrifolia. } \\ \text { Herl Paris or True- } \\ \text { love. }\end{array}\right\}$ Spreading. 


\section{A $\mathrm{L} \quad \mathrm{Y} \quad \mathrm{X}$.}

RE F LEX U M.
Fig. I.
Reflexed. $\quad\left\{\begin{array}{l}\text { Echinops Jpharocephalus. } \\ \text { Globe thiftle. }\end{array}\right.$

I N F L, A T U M.
Fig. 2. Inflated. LAlder-leav'd Hermannia.

ABREVIATUM.
Fig. 3.
Abreviated. \{

L. O N G U S.
Fig. 4.
Long. $\quad\left\{\begin{array}{l}\text { CEnothera biennis. } \\ \text { Tree Primrofe. }\end{array}\right.$

O B T U S U M.
Fig. 5.
Obtufe. $\left\{\begin{array}{l}\text { Rhodiola rofca. } \\ \text { Rofe Tree. }\end{array}\right.$

A C U T U M.
Fig. 6.
Acute. $\quad\left\{\begin{array}{l}\text { Rumex crijpa. } \\ \text { Great Curled Dock. }\end{array}\right.$

S P I N O S U M. \{Centauria Calcitrapa.

Fig. \%.
Spinous.

ACULEATUM.
Fig. 8.

Prickly. TTeazel.

$S$ U P E R U M. Camna Indica. I Standing thove the Superoule. Indian flowering reed. $\int$ Germen. I N F E R U S.
Fig. : 0.
Inferons. $\quad\left\{\begin{array}{c}\text { Euphorbia lathyrus. } \\ \text { Burning thorny Plant, } \\ \text { or Spurge. }\end{array}\right\} \begin{gathered}\text { Standing below the } \\ \text { Germen. }\end{gathered}$

C O \I MUNE. / Fig. $\%$, II, I2, I6, containing many FlowCommon. $\{$ ers, as in the compound Flowers.

IMBRICATUAI. f Fig. 7, 8, I , I 2, various Scales lying over one lmbricated. $\quad\{$ another like Tiles on a Houfe. 


\section{$\left[\begin{array}{ll}71 & ]\end{array}\right]$

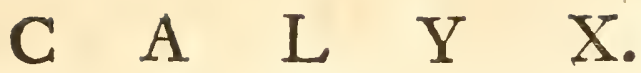

SQUARROSUM. $\{$ Conyza fqnarrofa.
Fig. 11.

Squarrous.

\{Great Flea-bane. $\int$ many ways.

SCARIOSUM.

Fig: I2.

Scarious.

$\left\{\begin{array}{l}\text { Centourea orientalis. } \\ \text { Oriental Centaury. }\end{array}\right\} \begin{aligned} & \text { The margins Mem- } \\ & \text { branaceous, Dry, } \\ & \text { Sounding } \\ & \text { touched. }\end{aligned}$

TURBINATUM.

Fig. 13 .

Turbinated

$\left\{\begin{array}{l}\text { Polygonum fagopyrum } \\ \text { Buck Wheat, }\end{array}\right\}$ Shaped like a Top.

G I B B U M.

Fig. 14.

Gibbous.

$\left\{\begin{array}{l}\text { Lunaria Annua, } \\ \text { Moon Wort, or Ho- } \\ \text { nefty. }\end{array}\right.$

CYLINDRICUM. $\{$ Erigeron ficulum.
Fig. 15.

Cylindric.

\{Red-Atalk'd Erigeron. $\}$ Forming a Cylinder.

CALYCULATUM. Prenanthes purpurca.
Fig. 16. Caliculated. $\quad$ Purple Prenanthes. $\}$ clesthe larger Calys. 


\section{[ 72 ] \\ I N V O L U R U M.}

An Involucrum is a kind of Calyx, ftanding remotc trom the Flower.

UNIVERSALIS. $\{$ Seinum faluftre.
Fig. I. Fig. I.
Univerfal. $\quad\left\{\begin{array}{l}\text { Standing under the } \\ \text { univerfal Unbel }(a .)\end{array}\right.$ PAR TI A L IS.

Fig. 2.
Partial. Fig. r. (b.) Standing under the partial Umbel.

PRO PR I U M. $\{$ Pafifiora corulea.
Fig. 3. Propicer. IBlue Paffion Flower. $\int$ Flowers. 


\section{( 73$)$ \\ G L $\quad$ U $\quad M \quad A$.}

A Hurk, a Calyx or Cup belonging to Graffee, whofe Flowers it embraces with the Velves folded over.

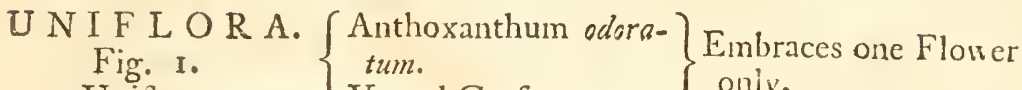
Uniflorus. Vernal Grafs. $\quad$ oniy.

MULTIFLORA.
Fig. 2. Multiflorous. Wild Oats. $\int$ ny Flowers.

U N I V A L V I S. \{ Scirpus lacufris. Fig. 3.
Univalve. $\quad\left\{\begin{array}{c}\text { Tall Clubb, or Bull } \\ \text { Rufh. }\end{array}\right\} \begin{aligned} & \text { When there is con } \\ & \text { ftantly one Scale. }\end{aligned}$ Bivalve.

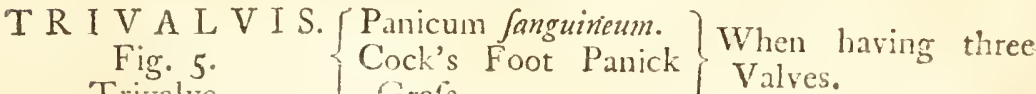
Trivalve. Grats.

MULTIVALVIS. Fig. 6. Multivalyc. $\{$ Bobartia Indica. $\}$ Having many Valres

COLORATA.

Fig. 7.

G L A B R A. Mat Grafs. Smooth.

H I S P I D A. Fig. 8. Hilpid. II U D I C A.
Fig. 9.
Mlude. $\quad\left\{\begin{array}{l}\text { Milium effusum. } \\ \text { Millet Grafs. }\end{array}\right\} \begin{aligned} & \text { Witlrout Points, or } \\ & \text { Arifta. }\end{aligned}$ $\{$ Bobortia, covered with hard Hairs. A R I S T A.
Fig. Io.
beard. 


\section{(73) \\ G L U M A.}

TERMINALIS.
Fig. II.
Terminal. $\left\{\begin{array}{l}\text { Stipa pennata. } \\ \text { Feather Grafs. }\end{array}\right\}$ Terminating the Hufk

I O R S A L I S. A Anthoxanthum odora- Fixed on the Back of Fig. I2.
Torfal. $\quad\left\{\begin{array}{c}\text { tum. } \\ \text { Vernal Grafs. }\end{array}\right\}$ the Hufk.

T O R T I L I S. (Avena fatua. Fig. 13.
iortil. 


\section{$\left[\begin{array}{ll}74 & ]\end{array}\right.$}

\section{AMENTUM and SPATHA.}

$\underset{\text { A M E N T IU M. I. }}{\text { Catkin. }}\left\{\begin{array}{l}\text { Coryllus avelana. } \\ \text { Hazel Nut Tree. }\end{array}\right\}$ A common Receptacle Spatha, a Sheath, a kind of Cup or Calyx, burfing lengthwife.

U N I V A L V IS. 2.
Univalve. $\left\{\begin{array}{l}\text { Arum maculatum. } \\ \text { Wake Robin. }\end{array}\right\}$ Having one Valve. B I V A L V I S. $\underset{\text { Fig. 3. }}{\text { Bivalve, or Dimidiate. }}\left\{\begin{array}{l}\text { Butomus umbellatus. } \\ \text { Water Gladiolus. }\end{array}\right\}$ Having two Valves.

Calyptra, a Veil or Hood, covering the Anthera of Mofies. R E C T A. Fig. 4 . Rect.

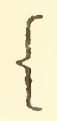

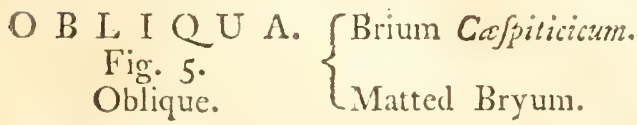

Volva, a membranaccous Calyx, proper to the Funge. APROXIMATA.
Fig. 6.
Aproximate. $\left\{\begin{array}{l}\text { Agaricus compeftes. } \\ \text { Field Miufhroom. }\end{array}\right\}$ Clofe to the head.

RE $\mathrm{NIOTA}$ A. $\mathrm{T}$.
Remote. 


\section{$\left[\begin{array}{ll}75 & ]\end{array}\right.$

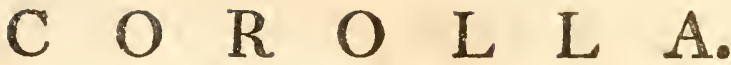

Corolla, is the Termination of the inner Bark, is prefent in the Flower e. g. MONOPEDULA,
Fig. I. Sigelia Anthelmia.

One Petal. $\quad$ Annual Worm Grafs.

B I P E T A L U S. Atraphaxis Spinofa.

Fig. 2.
Two Petals. $\quad\left\{\begin{array}{c}\text { Prickly Branched } \\ \text { Atraphaxis. }\end{array}\right.$

TRIPETALUS. $\quad$ Tradefontia Virginic

Fig 3.
Three Petals. $\quad\left\{\begin{array}{c}\text { na. } \\ \text { Spider Wort. }\end{array}\right.$

TETRA PETALUS. (Cheiranthus Incanus.

Fig. 4. $\quad$ Sour jetals.

QUINCUEPETALUS Cratzgus Aira.

Fig. $5 \cdot$
Five Petals. White Bean Tree.

HEXA PETALUS. S Naciffus pocticus.

Fig. 6.

Six Petals. White Narciffuc

MULTI PETALLS. 「Adonis virnalis.

Tig. $7 \cdot$
Many Petuls,

"T UBUL OS US. S Pimula officinal:

Fig. 8 .

Tubulous.

Cowflip. (a.)

L I M B US.

The limb. $\quad$ Fig. $8,9,(\ell$.

U N $\mathrm{N}$ U I S.
The Claw.

$\underset{\text { Regular. }}{\text { RE IS }}\left\{\begin{array}{l}\text { Fig. 4. 5, 6. } \\ \text { E A A I }\end{array}\right.$

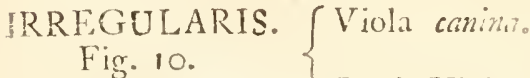

Trregular.

Dog's Ticiet. 


\section{[ 75 ] \\ G O R O L L}

I N E QU A L I S. \{ Rhodora Canadenfis. Fig. II.

Unequal. Cunadian Rhodora.

G L O B O S A.
Fig. I2.
Globous. $\quad\{$ Erica.

CAMPANULATA. (Campanula tracbelium.

Fig. 13. Canterbury Bell.

INFUNDIBILI-

FORMIS.
Fig. I 4. $\left\{\begin{array}{l}\text { Symphitum officinalis. } \\ \text { Common Comfiry }\end{array}\right.$

Infundibiliforme. Common Comfrey.

EISPOCRATIFORMIS. (Kalmia angufifolia.

Fig. 15.

Saiverthaped. mia. 


\section{$\left[\begin{array}{ll}7^{6} & ]\end{array}\right.$ \\ C O $\mathrm{R} O \mathrm{O}$ L $\mathrm{L}$.}

R O T A T A.
Fig. I.
Rotate. $\left\{\begin{array}{l}\text { Solanum P/uedo "cap- } \\ \text { ficum. } \\ \text { Winter Cherry. }\end{array}\right\}$ Wheel Ahaped.

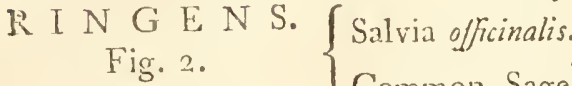

Ringent.
Fig. $3 \cdot$ PERSONATA. $\left\{\begin{array}{l}\text { Aconitum napcllus. } \\ \text { Common Wolf's bane. }\end{array}\right\}$ Gaping.

Fig. 4.
Mafqued. $\quad\left\{\begin{array}{l}\text { Digitalis purpurea. } \\ \text { Purple Fox Glove. }\end{array}\right.$

C R U C I A T A. $\{$ Cheiranthus incanus.

Fig. 5 .

Crols-haped. Stock July Flower.

C O N C A A.

Fig. 6.

Concave.

PATENS.

Spreading.

$\left\{\begin{array}{l}\text { Cralægus aira. } \\ \text { White Bean Tree. }\end{array}\right.$

Fig. 5, 6, 8, 10.

3 PPLLIONACEUS.

Fig. 7 .

Papilionaceus.

COMIPOSITA.

Fig. 8.

Compofite.

$\{$ Lathyrus Latijolia.

L I UU U, A T A.

Ligulate.

Fig. 9.

TUBULOSA.

Tubulous.

Everlanting Pea.

YAfter Chiweng is.

China After.

$\left\{\left(a_{0}\right)\right.$ Tongue Shaped. After, confifting of Ligulate and Tubulous Florets.

IMBRICATA.

Fig. IO,

Imbicated.

$\{$ (6.) the Floret's TuQ bulous.

$\{$ Leontodon taraxacum.

Dandelion.

CONYZA Squarofa. $\left\{\begin{array}{c}\text { An agragat Flower, confifting of Toubulous } \\ \text { Fig. 11. }\end{array}\right.$

Plowman's Spikcnard. E Elowers. 


\section{$(77)$ \\ $N$ E G T A R I U M.}

Is that part of a Flower that bears the Honey, or Meliferous Juices.

PR O PR I U M. Fig. I. Proper. Naciffus Daffodil. $\}$ part of the Flower.

CORNICULATUM.
Fig. 2. $\left\{\begin{array}{l}\text { Aquilegia. } \\ \text { Columbine. }\end{array}\right.$

Horn Shaped. Columbine.

P E T A L I N U M. CRanunculus bulbofus.

Fig. 3. $\quad$ Crowfoot, or Butter

On the Petals. Cup.

KIGGELARIA.

Africana.

Fig. 4 .

African Kiggelara.

TRITELARIA Co-

rona Imperialis.

Fig. 5 .

Crown Imperial.

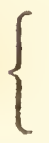

PARNASSIA Paluf-
tris.

Fig. 6.

Grafs of Parnaffus.

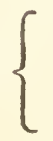

NERIUM Oleander.

Rofe Bay.

Fig. 7 .

RA D I A T ED.
Fig. 8.
In a Ray. $\quad\left\{\begin{array}{c}\text { Paffifora carulea: } \\ \text { Common Paffon } \\ \text { Flower. }\end{array}\right.$

PEDUNCULATUS. f Aconitum napcllus.

Peduncled. Aionkthood. 


\section{$\left(7^{8}\right)$}

\section{STAMENS and A N THERA.}

Are the Male part of a Flower, is vifcus, preparing the Polien or Farina, and confifts of Filoments and $A n-$ the'a.

FILAMENTUM.

Fig. 1 .

Filament.

EE C A L I S. $\{($ a. $)$ as in Tulips.
Eig, 2.
Equal. IN $\mathrm{E}$ QU A L I A.
Fig. 3. Cheiranthus Incanis.

Unequal, Stock July Flower.

D I D O. $\left\{\begin{array}{l}\text { Melittis Melifiphyllis. } \\ \text { Falm-leav'd Melifi- } \\ \text { phyllis. }\end{array}\right.$

C O N N A T A. $\underset{\text { Fig. } 5 .}{\text { United. }} \quad\left\{\begin{array}{l}\text { Geranium Sfricanum. } \\ \text { African Geranium. }\end{array}\right\}$ And in Fig. 6.

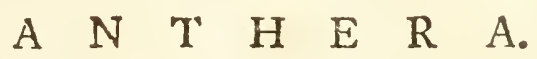

DIS T I N C T A. $\left\{\right.$ Fig. I, 2, $\left(a_{0}\right) 3,4,5$, not cohering.

C O N A T A.
Fig.t.
United. $\quad\left\{\begin{array}{l}\text { Lobelia Cardinalis } \\ \text { Carinin Flower. }\end{array} \quad\left\{\begin{array}{l}\text { Joined by the fides in } \\ \text { to one body. }\end{array}\right.\right.$

INCUMBENS..
Fig. 7.
Incrm! unt. $\{$ As in Lillics.

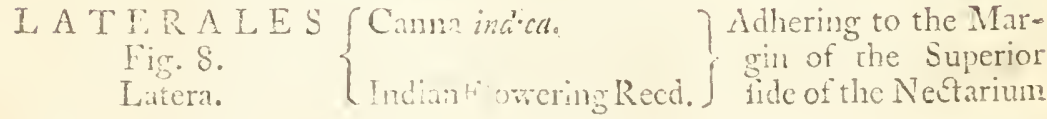

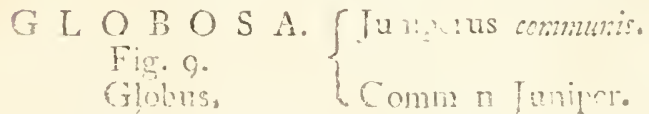




\section{( 78$)$}

\section{A $N$ ' $T$ H}

D I D I M Æ.
Fig. 10.
Twins. $\left\{\begin{array}{l}\text { Salix. } \\ \text { Willow. }\end{array}\right.$

S U B U L A T A.
Fig. II.
Awl-hhaped. $\{$ Fig. I, 2, (b.)

SAGITTATATÆ..
Fig. I
Arrow-fhaped. $\{$ Cracus.

B I C O R N I S. S Erica abietina,

Fig. I3.
Two Horned.

E R U P T U S. Melittis melifiphyllus. D Difcharging the ForFig. 14. Burfting. $\left\{\begin{array}{c}\text { Balm-leav'd Melifi- } \\ \text { phyllis。 }\end{array}\right\} \begin{aligned} & \text { ma with an elaftic } \\ & \text { motion. }\end{aligned}$ 


\section{(79) \\ $\mathrm{P} I \mathrm{~S}$ T L L U M.}

The Pifillum, a vifcous Humour adhering to the Fruit for the reception of the Pollen, and is the Female Organ of Generation. The Germen is the Embryo or Rudiment of the Fruit, yet immature.

The Stylus, is the Part that elevates the Stigma from the Germen.

The Stigma, is the Summit or apex of the Riftillum, covered with a Menftruous moifter, that breaks and diffolves the Pollen, and fits it for impregnation of the Germen.

$\left.\begin{array}{l}\text { S U P E R U M. } \\ \text { Fig. I. } \\ \text { Above the Corolla. }\end{array} \begin{array}{c}\text { Wachendorfia thyrfifo- } \\ \text { lia. } \\ \text { Simple ftalked Wach- } \\ \text { endorfia. }\end{array}\right\} \begin{gathered}\text { Included in the Co- } \\ \text { rolla. }\end{gathered}$ I N F E R U M.
Fig. 2.
Inferous. $\quad\left\{\begin{array}{l}\text { Enethera Biennis. } \\ \text { Tree Primrofe. }\end{array}\right\}$ Below the Corolla. PEDUNCULATUS. $\{$ Euporbia.

Fig. 3.
Pcduncled.y $\quad\left\{\begin{array}{l}\text { Etporbia. } \\ \text { Spurge. }\end{array}\right.$

FILIRFORMIS.
Fig. 4.
Filiforme. $\quad\left\{\begin{array}{l}\text { Nelittis melifiphyllus. } \\ \text { Balm-leav'd Melittis. }\end{array}\right\}$ Thread-Shaped.

SUBULATUS.

Fig. 5 .

Subulate.

CLA VATUS.

Fig. 6.

Club-fhaped.

ERECT U S.

- Erect.

DECLINATUS,

Fig. 7.

Declining.

ADECENAENS.

Fig. S.

Accunding.

Leucojum.

Fig. I, 2, 4, $9,10, \mathrm{Ir}$.

Nifolia.

Anthyllis tes as by lla

Kibney Vetch. 


\section{[ $\begin{array}{ll}79 & \text { ] }\end{array}$}

$\begin{aligned} & \text { S I M P L E X. } \\ & \text { Fig. 9. } \\ & \text { Simple. }\end{aligned}=\left\{\begin{array}{l}\text { Dipfacus. } \\ \text { Teazel. }\end{array}\right.$

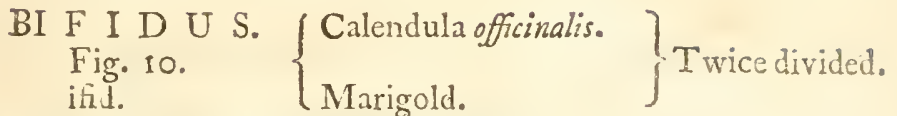

T R I F I D U S.
Fig. I1.
Trifid. QUADRIFIDUS.
Fig. I2.
Quadrifid. $\left\{\begin{array}{l}\text { Populus niger. } \\ \text { Black Poplar. }\end{array}\right\}$ Four times Divided.

QUINQUEFIDUS. \{ Geranium.
Fig. I 3 . Fig. I 3.
Quinquetid. Fire times Divided.

MULTIFIDUS.
Fig. I4.
Multifid. $\left\{\begin{array}{l}\text { Aleca rofea. } \\ \text { Hollyhock. }\end{array}\right.$ J Many times Divided.

PERFORATUM.
Perforated. Fig. IA, (a.) hollow.

C A P I T A T I S.
Fig 15.
Capitate. $\left\{\begin{array}{l}\text { Punica granatum. } \\ \text { Pomegranate. }\end{array}\right\}$ Having a Head. 


\section{[ 80 ] \\ PE R I A R P I U M.}

A Capfule or Seed veffel, is the Germen grown to Matit rity, big with Seeds, which when ripe it difcharges the Seeds.

C A P S U L A.
Fig. I.
Capfule. $\left\{\begin{array}{l}\text { Stellaria Holofeum. } \\ \text { Stitchwort. }\end{array}\right.$

V A L V U L A. $\{$ Fig. I, (a.)

Having three protoles.

UNILOCULARIS. $\{$ Fig. I1.
Unilocular.

BLLCULARIS.
Fig. 2.
Bilocular. $\quad\left\{\begin{array}{l}\text { LobeliaGardinalis, } \\ \text { Cardinal Flower. }\end{array}\right\}$ Two Cells.

TR I COE CA.

Fig 3 .

Trilocularis.

Trilocular.

Euphorbia.

Spurge.

$\int$ rent hinobs.

QUADRILOCULARIS.

Fig. 4.
Quadrilocular. Tree Primrofe.

\}One Celi.

QUINQUELOCULARIS

Fig. 5 .

Five Locular.

Sarracenia fava.

Yellow Side Saddle Five Cells.

SEXLOCULARIS.

Fig. 6.

Six Locular.

OCTOLOCULARIS.
Fig. 7 . Rhodiola rofers.

Eight Locular.

Fig. 8.

Nine Locular.

Citrus burantium.

Orange.

DLCEMLOCULARIS. J Linum UJitatifmus.

Fig. 9.

Ten Locular. F Flax. $\int \operatorname{Six} C e l l s$.

\} Eiglit Colis.
Nine Cells,

Ten Cells, 


\section{$[80$ ] \\ PER I C A R P U M.}

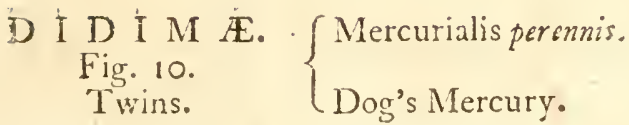

CIRCUMCISSA.
Fig. II.
Circumcifed. $\left\{\begin{array}{l}\text { Plantago major. } \\ \text { Rore Plantain. }\end{array}\right\} \begin{aligned} & \text { Parting horizontally } \\ & \text { in the iniddle }\end{aligned}$

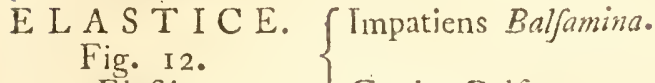

Elaftic. $\quad$ Garden Balfam.

I N F L A T I S. Colutea arborefcens.

Fig. I 3 . Intiated.

$\left\{\begin{array}{l}\text { Common Bladder fen- } \\ \text { na. }\end{array}\right.$ 


\section{(81) \\ PER I C A R P I U M.}

SIL I QU A. Fig. I.

A Shell.

$$
\left\{\begin{array}{l}
\text { Cheiranthus Incanis. } \\
\text { Stock July Flower. }
\end{array}\right\} \begin{aligned}
& \text { A Pericarpium of two } \\
& \text { Valves, in which the } \\
& \text { Seeds are fixed alter- } \\
& \text { nately to the two op. } \\
& \text { fite Sutures. }
\end{aligned}
$$

$\underset{\text { Sig. 2. }}{\text { S I I. I C U A. }}\left\{\begin{array}{l}\text { Fumaria Lutea. } \\ \text { Yellow Fumatory. }\end{array}\right\}$ A little Bod.
T O R UL C S A. \{Raphantus:
Fig. 3.
Radifh.
$\left\{\begin{array}{l}\text { Brawni Protuberances, } \\ \text { when the Pericarpi- } \\ \text { umis bunched out by } \\ \text { the Seeds. }\end{array}\right.$

$\underset{\text { Fig. 4. }}{\text { ARTICULATUS. }} \underset{\text { Articulated. }}{\text { Hypocoum proeumbes. }}\}$ In Joints.

PARALI.ELUM.U.
Parallel. Fig. 1.

TRANSVERSUM
DISSEPIMENTUM.
Fig: 5 .
The Diffepiment run-
ing croflwife. $\left\{\begin{array}{c}\text { Polygala Myrtifolia. } \\ \text { Myrtle-leav'd Polyga- } \\ \text { la. }\end{array}\right\}$ $\left.\begin{array}{l}\text { LE G U M E N. \{ Lathyrus latifolia. } \\ \text { Fig. 6. }\end{array}\right\} \begin{aligned} & \text { Two Valved, the } \\ & \text { Seeds fixed to one Su- }\end{aligned}$ A Shell. Everlafting Pea. ture only.

ISTHMUS INTER
CEPTUM. $\left\{\begin{array}{l}\text { Pods 'xith'variouscrofs } \\ \text { divifions, forming di- } \\ \text { ftinct cells, as in Fig. } \\ 5, \text { and } 8 .\end{array}\right\}$

SCORPIURUS

Vermiculate.

Common Caterpiliar.

CERATONIA Siliqu 1.

St. John's Bread. 


\section{$\left[\begin{array}{ll}82 & \text { ] }\end{array}\right]$}

\section{PER I C A R P I M.}

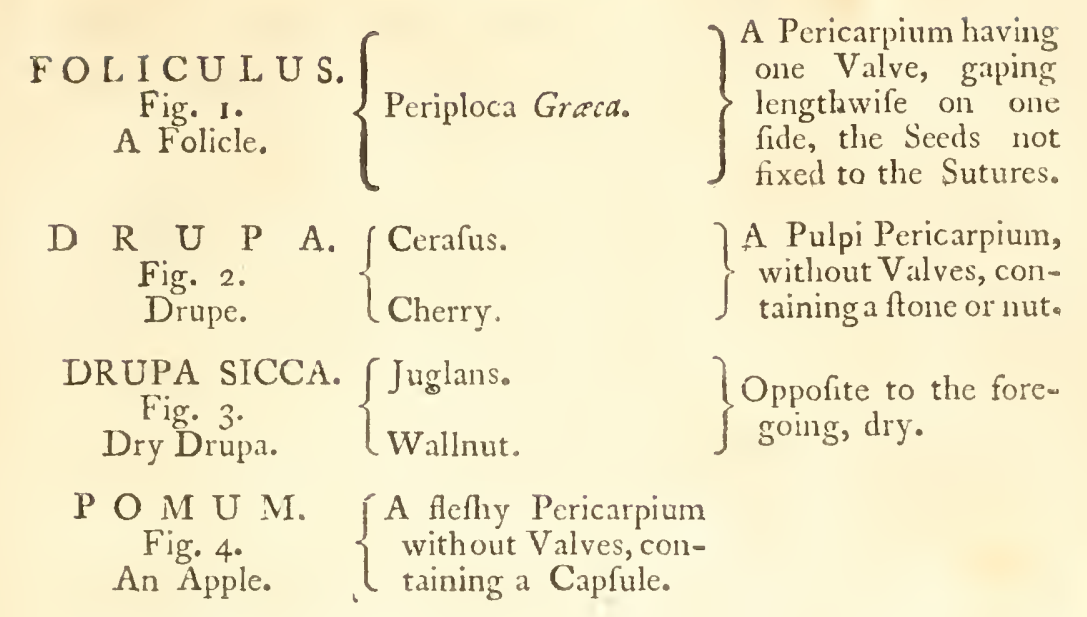

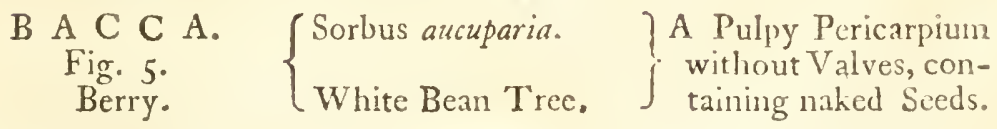
NIDULANTIA.
Fig. 6. $\quad\left\{\begin{array}{l}\text { Ribes crofularia. } \\ \text { Goofe Berry. }\end{array} \quad\left\{\begin{array}{l}\text { The Seeds nefiling in } \\ \text { the pulp of the Berry. }\end{array}\right.\right.$

S T R O B I L U S. \{ Pinus fyluefris. $\quad$ A Pericarpium form-

Fig. 7.
A Cone. ed from an Amertum
wit hhard Scales. 


\section{$(83)$

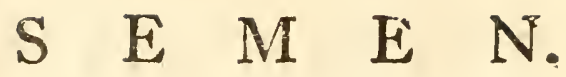

Seed, the Rudiment of a new Plant; are known according to the Number, Figure, Superfices and Confiftence.

צ. HILUM, the Eye, an external Scar of the Seed, where it has been fixed to the Fruit or Recepticle, as in the Bean.

2. CORCULUM, The Effence of a new Plant within the Seeds, c. b.

3. PLUMULA, a part of the Corculum, the afcending Scaly Part of the Plant.

ROSTELLUM, the defcending part of the Corculum that forms the Root. (c.)

COTYLEDON, the fide Lobess of the Seed, of a parous fubftance, and perifhes ${ }^{\circ}\left(d_{\text {. }}\right)$ Fig. 2, 3.

C O R O N A.
Fig. 4.
Crown. $\quad\left\{\begin{array}{l}\text { Dipfacus fullonum. } \\ \text { Wild Teazel. }\end{array}\right\} \begin{aligned} & \text { A little Cup, adher- } \\ & \text { ing to the top of the } \\ & \text { Sced, is deciduous. }\end{aligned}$

P A P P U S.

Fig. 5 .

Pappous.

$\left\{\begin{array}{l}\text { Carduus. } \\ \text { Thiftle. }\end{array}\right.$

$\left\{\begin{array}{l}\text { A downy feathered } \\ \text { Cup, adhering to the } \\ \text { Top of the Seed, by } \\ \text { which it flies. }\end{array}\right.$

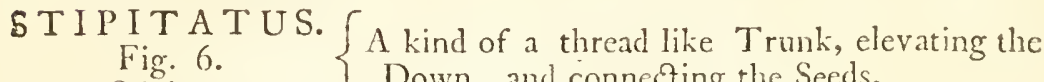
Stipitate Down, and connecting the Seeds. Chaffy. $\quad$ Annual Sun Flower. $\}$ are deciduous. CAP ILLA R I S.
Fig. 8 . Hicrachium Pilogella 0 Hairy: $\left\{\begin{array}{l}\text { Moufe-ear Hawkweed }\end{array}\right\}$ Simple undivided hairs

P L U MOS U S.
Fig. 9.
SLumous. $\quad\left\{\begin{array}{l}\text { Cnicus oleraceus. } \\ \text { Pale-fowered Cnicus. }\end{array}\right\}$ Feathery Hairs. 


\section{[ 83 ]

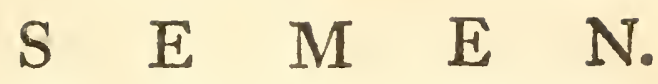

C A U D A.
Fig. IO.
Tail. $\quad\left\{\begin{array}{l}\text { Atragena Alpina. } \\ \text { Alpire Atragena. }\end{array}\right\}$ Having a feathery tail

N A M U S.

Fig. II.

Nooked.

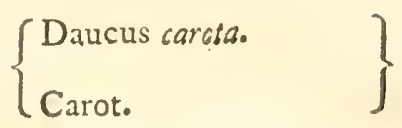

A R I L L U S.
Fig. I2.

\begin{tabular}{|c|c|c|}
\hline $\begin{array}{l}\text { A L A. } \\
\text { Fig. 13. } \\
\text { Wing. }\end{array}$ & $\left\{\begin{array}{l}\text { Pinus Jylveftris. } \\
\text { Scotch Fir. }\end{array}\right.$ & $\begin{array}{l}\text { A Membrandceous } \\
\text { Wing, fixed to the } \\
\text { Seed. }\end{array}$ \\
\hline Fig & $\begin{array}{l}\text { Platanis Oriantalis. } \\
\text { Crienta Paln Tree. }\end{array}$ & \\
\hline $\begin{array}{l}N \text { U X. } \\
\text { Fig. 15. } \\
\text { Nut. }\end{array}$ & $\left\{\begin{array}{l}\text { Coryllus Avellana. } \\
\text { Hazel Nut. }\end{array}\right.$ & $\begin{array}{l}\text { Seed, covered } \\
\text { bony epitermu } \\
\text { Shell. }\end{array}$ \\
\hline
\end{tabular}




\section{$(8+)$ \\ R E C E P T A C ULU M.}

'The Receptacle is the Bale, by which the Parts of Fruetification are cornected.

COMMUNE.
Fig. I.
Common. $\quad\left\{\begin{array}{l}\text { Calendula officinalis. } \\ \text { Common Marigold. }\end{array}\right\} \begin{aligned} & \text { Containing many } \\ & \text { Flowers and Fruit, as } \\ & \text { alfo in Fig. I, 2,3. }\end{aligned}$

PUNCTATUM. [Leontodon Taraxacum.
Fig. 2. Punctated. Dandelion.

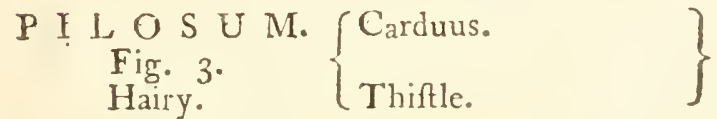

PALEACEUM.
Fig. 4.
Paleaceous. $\quad\left\{\begin{array}{l}\text { Rudbeckia purpurea. } \\ \text { Purple Rudbeckia. }\end{array}\right\} \begin{aligned} & \text { Chaffy Scales, which } \\ & \text { diftinguifh the Flow- } \\ & \text { ers. }\end{aligned}$ P L A N U M. $\{$ Fig. I.

C O N I C U M. $\left\{\begin{array}{l}\text { Dipfacus fulonum. } \\ \text { Fig. 5. } \\ \text { Conic. }\end{array}\right\} \begin{aligned} & \text { Cone fhaped, round, } \\ & \text { leffening towards the } \\ & \text { point. }\end{aligned}$ SUB U L A T U M.
Fig. 6.
Sulbulate. $\quad\left\{\begin{array}{l}\text { Myofurus minimis. } \\ \text { Moufe Tail. }\end{array}\right\}$ Shaped like an Awlo F I. O R I S.
Fig. 7.8 $\left\{\begin{array}{l}\text { Of the Flower, } \\ \text { andl } \\ \text { of the Fruit. }\end{array}\right\} \begin{aligned} & \text { Rubus Fruticofis. } \\ & \text { Bramble. }\end{aligned}$

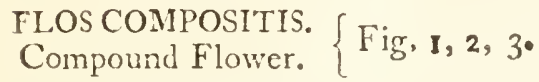

ILOS AGREGATIS. [Scabiofa fuciifa. The Receptacle is lon$\begin{array}{c}\text { Fig. 8. } \\ \text { Agregate Hlowers. }\end{array}$ Devil's-bit Scabious. $\}$ ger, the Flowers have 


\section{$(84)$ \\ RE C E P T A C U L M.}

Umbella, an Umbel, a Receptacle which from a common Centre runs out into Thread-Thaped Footfalks of Proportionate lengihs.

S I M P L E X.
Fig. 9.
Simple. $\quad\left\{\begin{array}{l}\text { Androface Seprentrio- } \\ \text { nalis. } \\ \text { foothed-leav'd An- } \\ \text { drofee. }\end{array}\right\} \begin{aligned} & \text { The Footftalks from } \\ & \text { one ccntre of the Re- } \\ & \text { ceptacle. }\end{aligned}$ COMPOSII A. Bupleururi rodundifo- When every Footftalk Fig. I0 $\quad$ lium, Compoite. $\left.\quad \begin{array}{l}\text { Hare's Ear, or Thorw } \\ \text { Wax. }\end{array}\right\} \begin{aligned} & \text { bel produces a par- } \\ & \text { tial Umbel. }\end{aligned}$ UNIVERSAIIS. (a.) Fig. 10, compofed of many little Umbels, Univerfal. I (b.) the Partial Umbel.

PRO L I F ERA.
Fig. II.
Paolifer. $\left\{\begin{array}{l}\text { CEnanthe crocata. } \\ \text { Hemlock. }\end{array}\right\} \begin{aligned} & \text { An Umbel more thian } \\ & \text { decompount. }\end{aligned}$

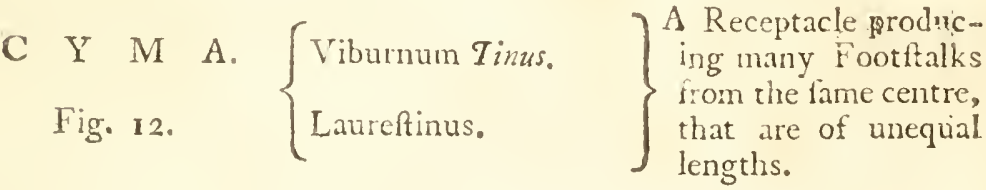
R A C H I S. \{Priànus fyluefris. A Receptacle, the Fig. 13. $\left\{\begin{array}{l}\text { Scotch Fir. } \\ \text { ed to it leigthwife, }\end{array}\right.$

S P A D I X. \{Arum maculatunt. A Receptacle, produFig. I4. $\left\{\begin{array}{l}\text { Wake Rooin. } \\ \text { War. }\end{array}\right\} \begin{aligned} & \text { ced within a Spatha } \\ & \text { or Sheath, is fimple, } \\ & \text { not divided. }\end{aligned}$

S P A D I X. \{ A Receptacle proper to the Balms, and is Fig. 15. $\{$ divided. 


\section{[ 85 ] \\ B U L B U S.}

A Bulb, is an Hibernacle placed on the defcending Caudex, and contains the Rudiments of the Plant and Leaf that perimes.

S O L I T U S.
Fig. I.
Solid. $\left\{\begin{array}{l}\text { Ranunculus brilbofus. } \\ \text { Butter Cup: }\end{array}\right\} \begin{aligned} & \text { A Solid flefhy Bulb; } \\ & \text { without any internal } \\ & \text { divifion. }\end{aligned}$ T U N I C A T U S.
Fig. 2.
Coated. $\quad\left\{\begin{array}{l}\text { Allium capa. } \\ \text { Onion. }\end{array}\right\} \begin{aligned} & \text { Coats lying over each } \\ & \text { other. }\end{aligned}$ SQUAMATUS.
Fig. 3.
Squamous. $\quad\left\{\begin{array}{l}\text { Lilium candidim. } \\ \text { White Lilly. }\end{array}\right\}$ Confifting of Scales.

C A U L I N U S.
Fig. 4.
Cauline: $\left\{\begin{array}{l}\text { Lilium bulbiferum. } \\ \text { Bulb-bearing Lilly. }\end{array}\right\} \begin{aligned} & \text { Growing on the Stem } \\ & \text { of the Plant: }\end{aligned}$ Gemma. a Bud, is an Hibernacle; with its Leaves, \&c. F O L I A R I S. \{ Betula alnus. Fig. 5 . Foliar. \} To Leaves only( a.) F L O R A L I S.
Fig. 6.
Floral. $\left\{\begin{array}{l}\text { Corylus avelana. } \\ \text { Hazel Nut. }\end{array}\right.$ \} ToFlowers only $(a$. C O M U N I S.
Fig. 7.
Common. $\quad\left\{\begin{array}{l}\text { Amigtalus Perfica. } \\ \text { Peach Tree. }\end{array}\right\} \begin{aligned} & \text { Common to both flow- } \\ & \text { ers and Leaves: }(a)\end{aligned}$ 


\section{$\left[\begin{array}{ll}86 & ]\end{array}\right.$ \\ $\mathrm{V} E \mathrm{E}$ N A T I O.}

Vernation is the manner how the Leaves are folded in the Gem or Bud, as feen.

Fig. ז. CONVOLUTE, Canna indica.

2. INVOLUTE, Alifina Plartago, Potamogeton, Viola.

3. REVOLUTE, Primula ver is R ofmarinus Nerium.

4. OBVOLUTE, Dianthus barbatus Lychnis Dipfacus.

5. CONVOLUTE, Prunus cerafus Lactuca Hierachium.

6. IMBRICATE, Campanula rodundifolia.

7. EQUI rANT, Iris Pfeudo, Acorus. Laurus. Daphne. Syringa: With two prominent Angles.

8. PLICATE, Veratum album. Malva. Urtica. Paffiflora.

9. CONVOlUTE, doubly, Arum maculatum. Melianthus Rofa: more than one leaf.

Io. INVOLUTE, ospofite. Pyrus Malus. Conmelina anma.

I. INVOLUTE, altenaoe.

12. REVOLUTE, oppofite.

I3. CONVOLUTE, doubly.

14. COVOLUTE, trebly.

35. CIRCINALE, Spirale.

EQUTTANT, ancipit. Equitant three ways, fo as to form a Triangle. 



\section{E R R A T}

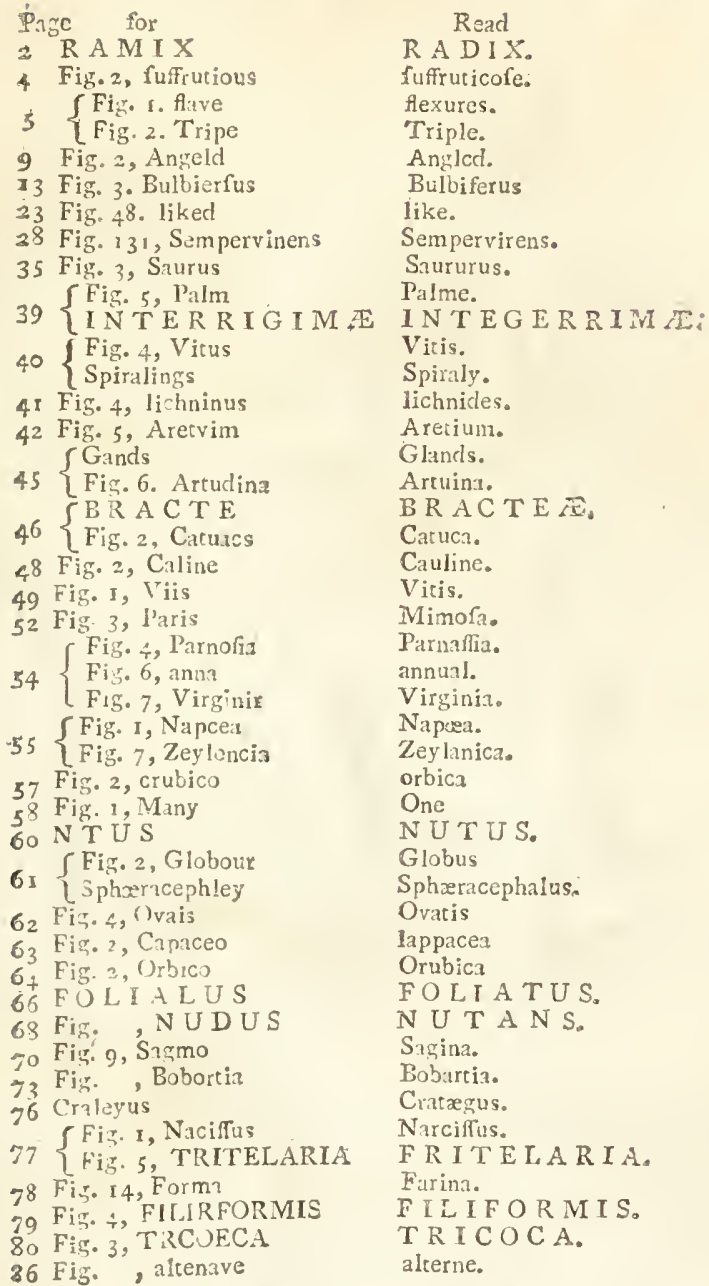

\section{O M I S I O N.}

Tib. 60.

Fig. 4.

\{ERACTEATUS. \}having foral leaves。

Tab. 71.

fwen the Cup, or Caiys, is

$\mathrm{T}_{\mathrm{ab}} .73$.

Fig. 7 .

Tabi, 74.

Eivg. 4 ;

$$
\begin{aligned}
& \{\text { COLORATA。 }\} \\
& \{\text { RICTA。 }
\end{aligned}
$$





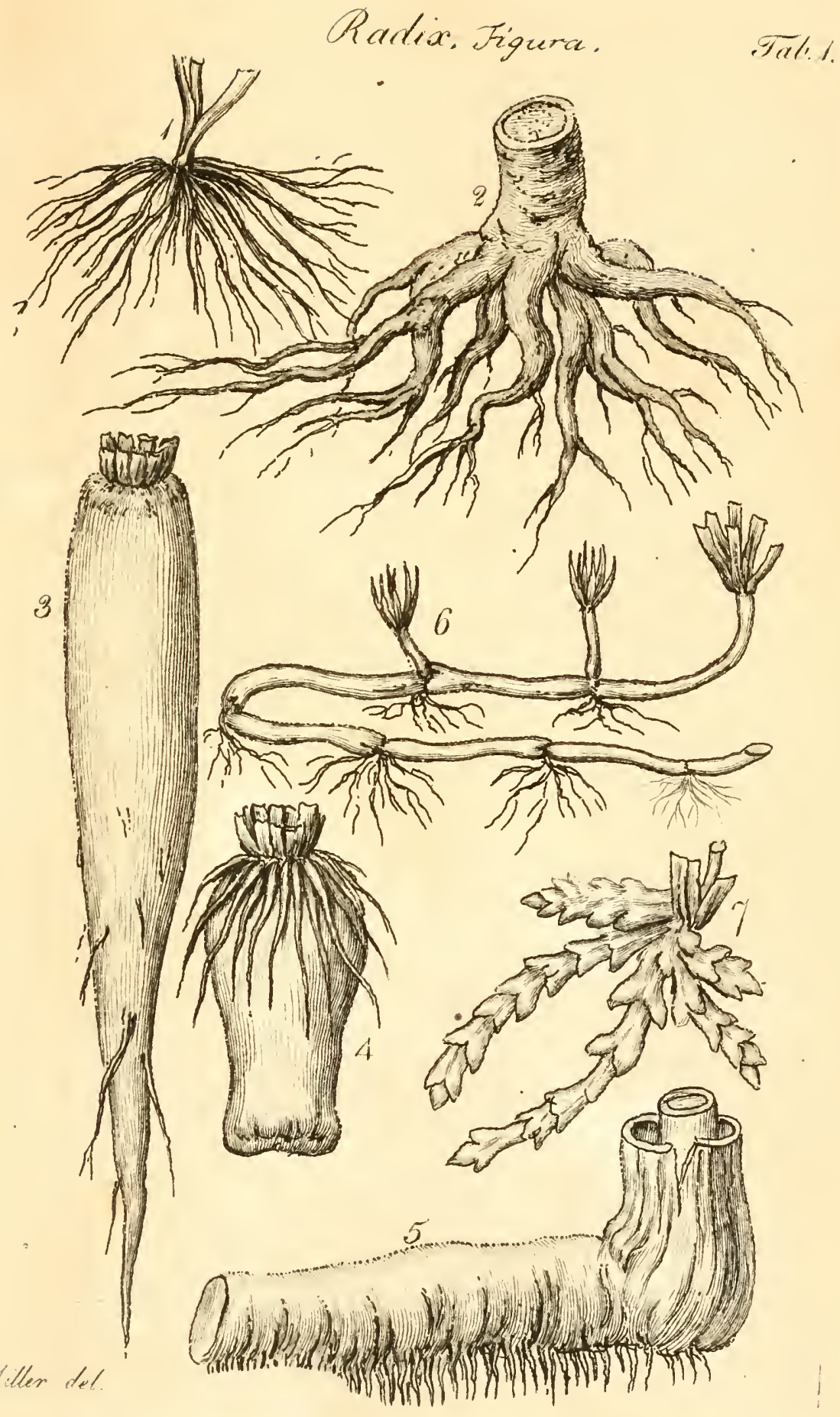





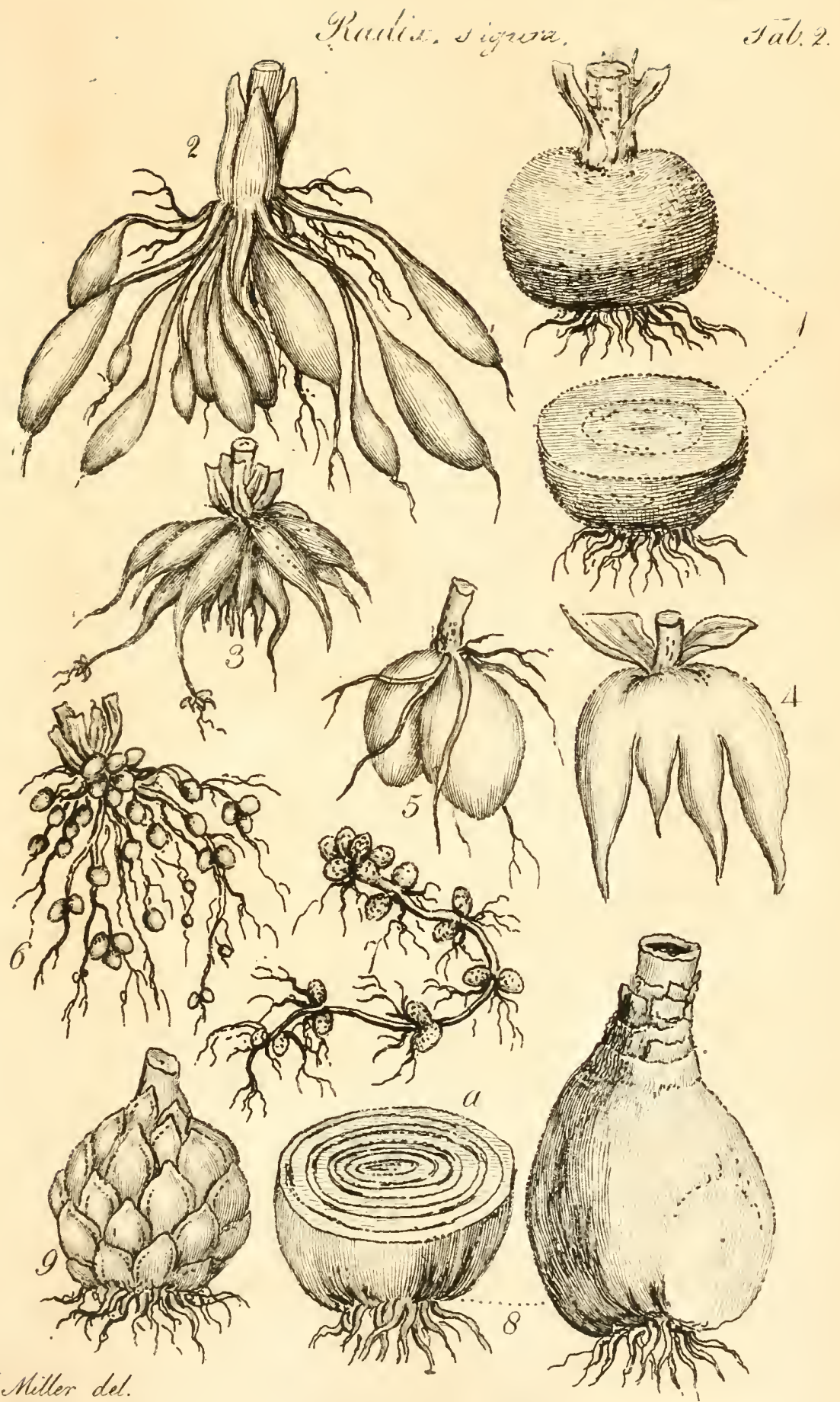





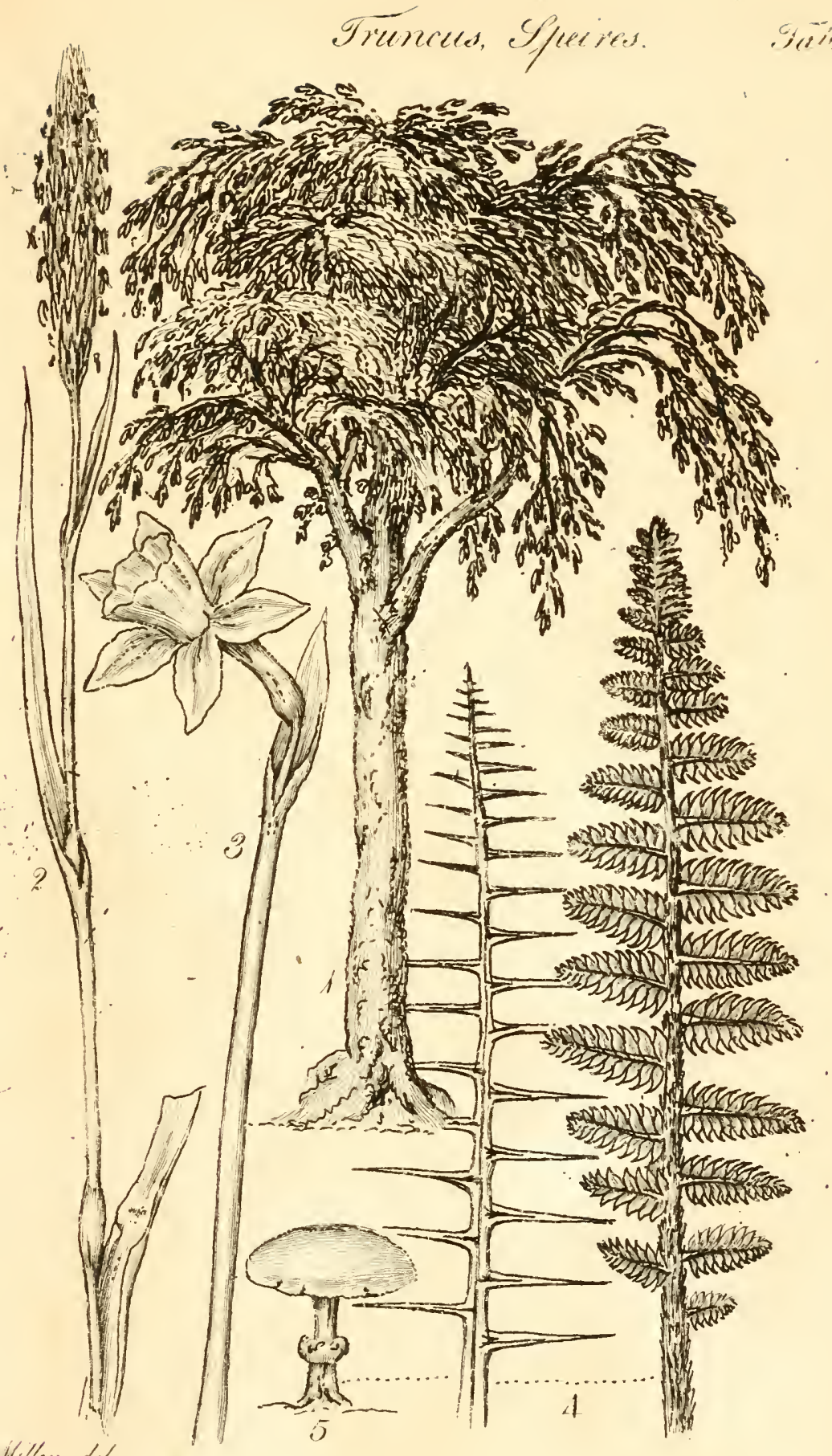



Gruncuer. Deratione. Tat.4.

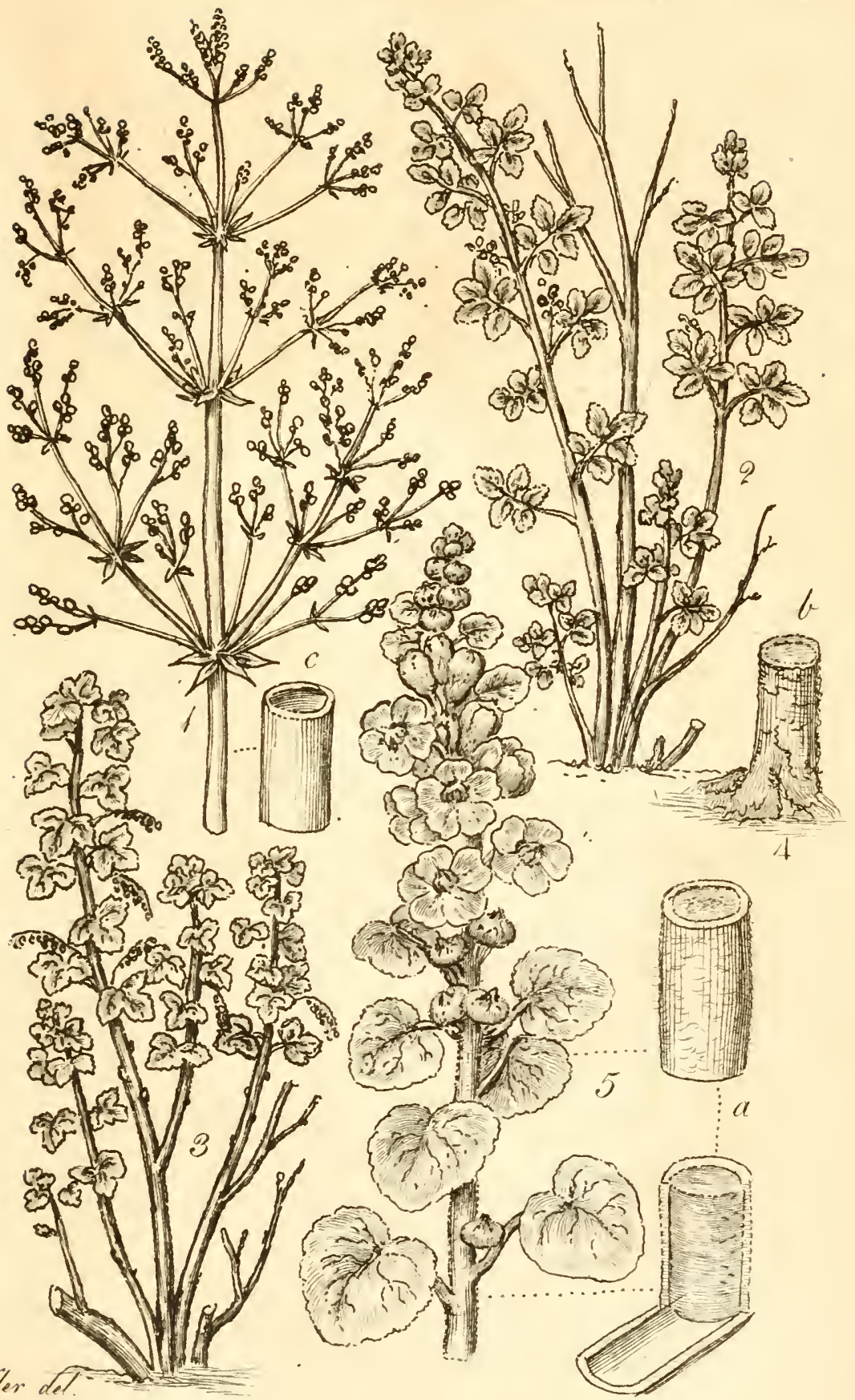





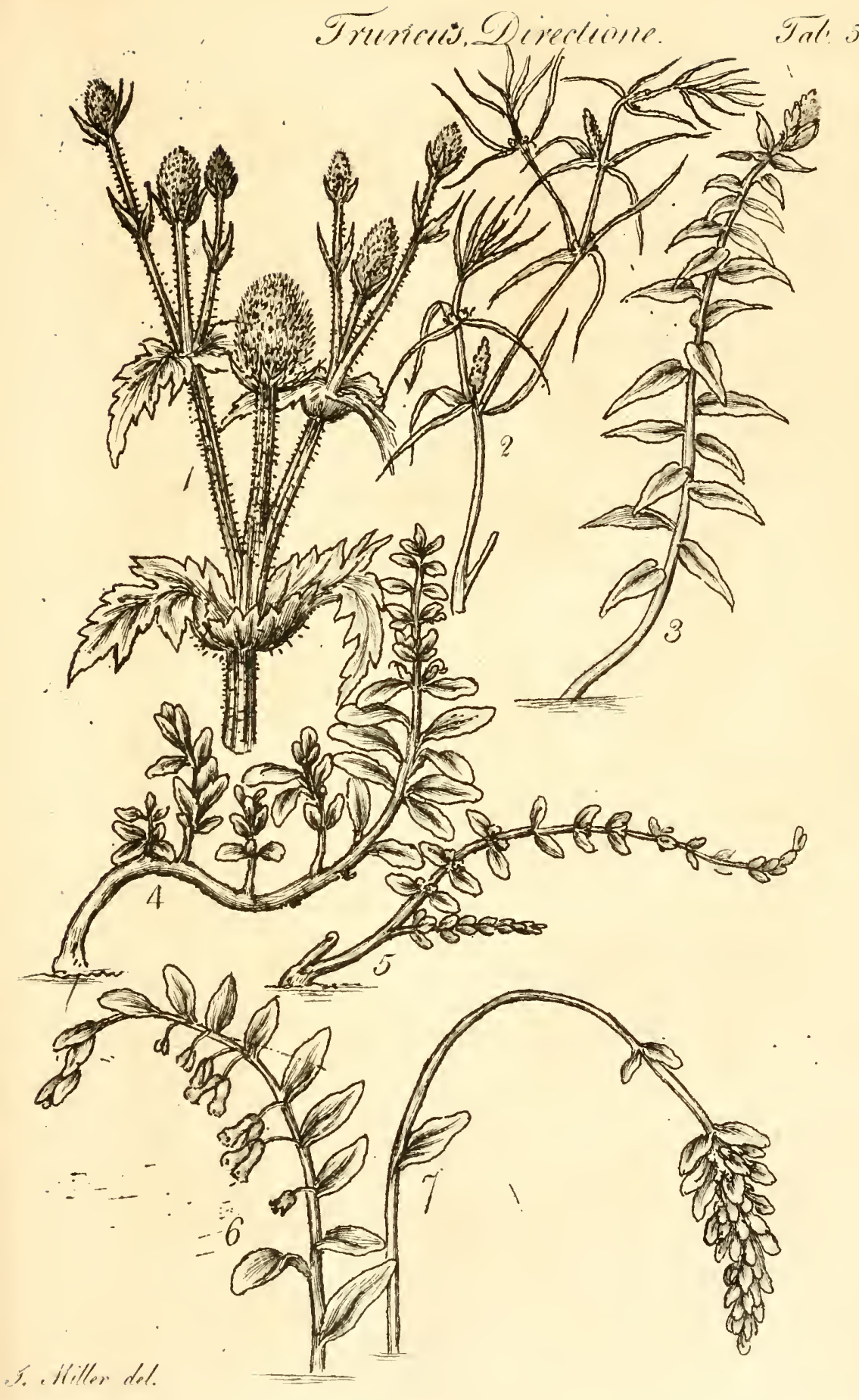



Fruncu, Directione.

Serl: 0

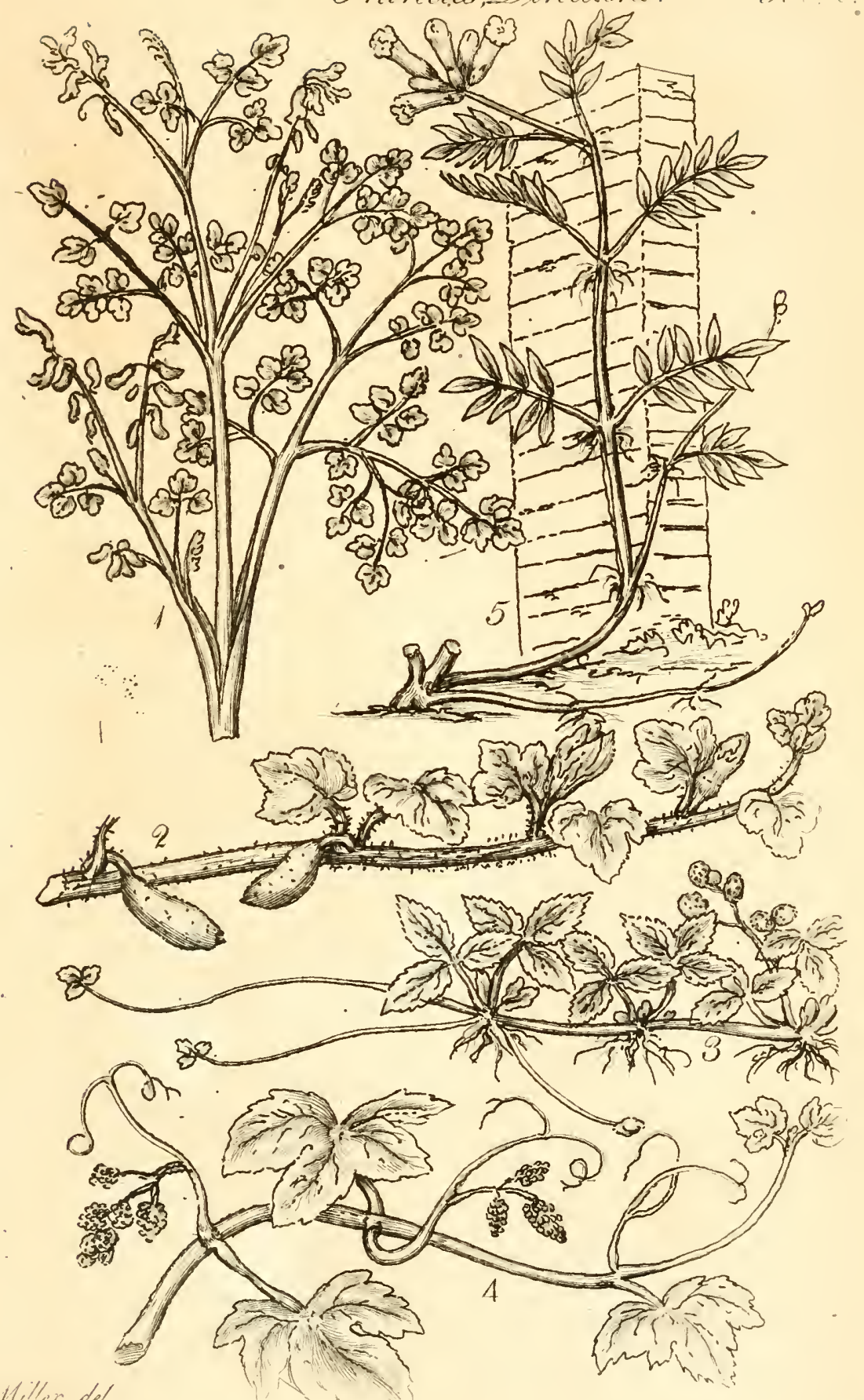



Grinncers, Livectione. Tret.

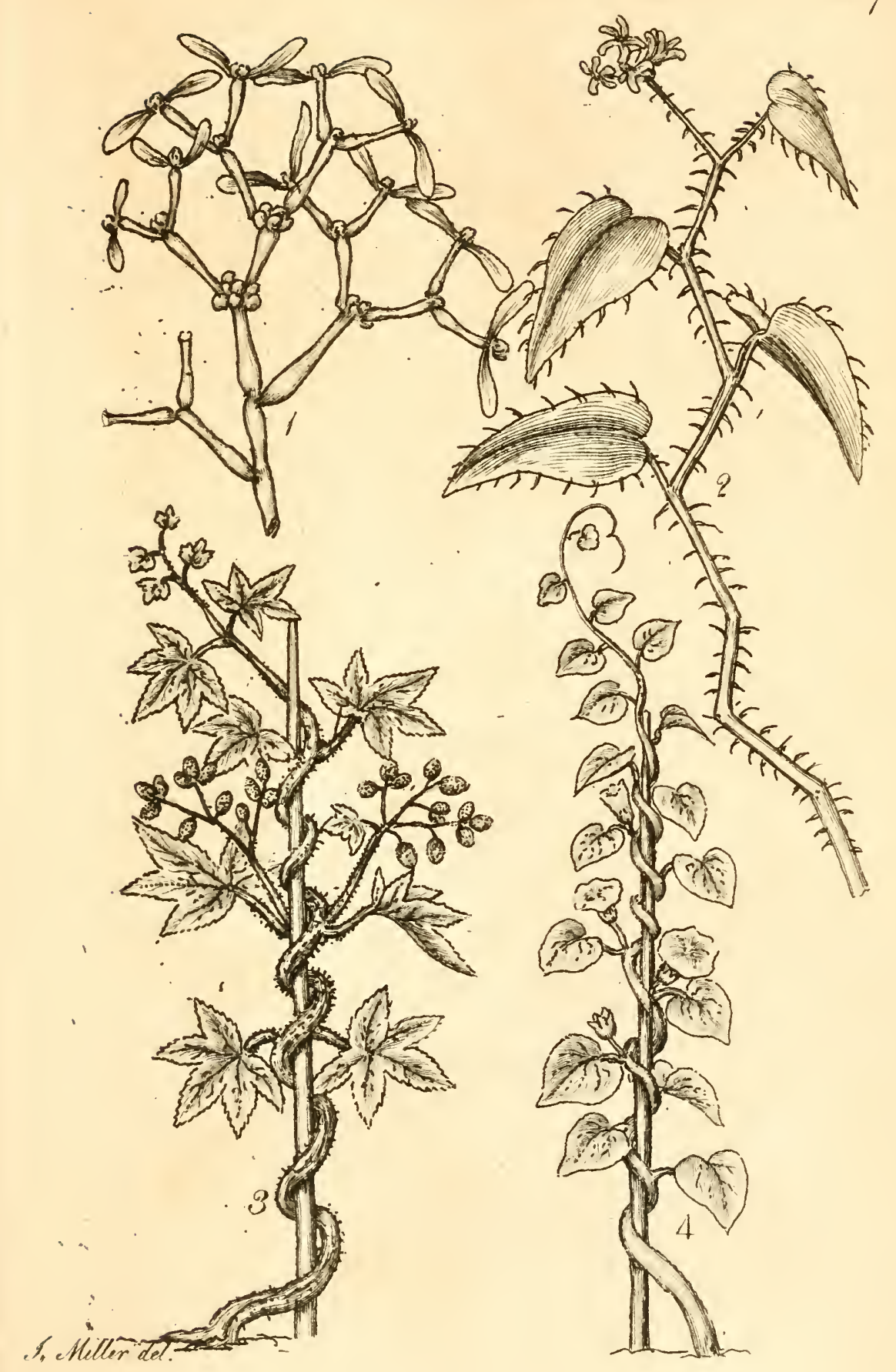





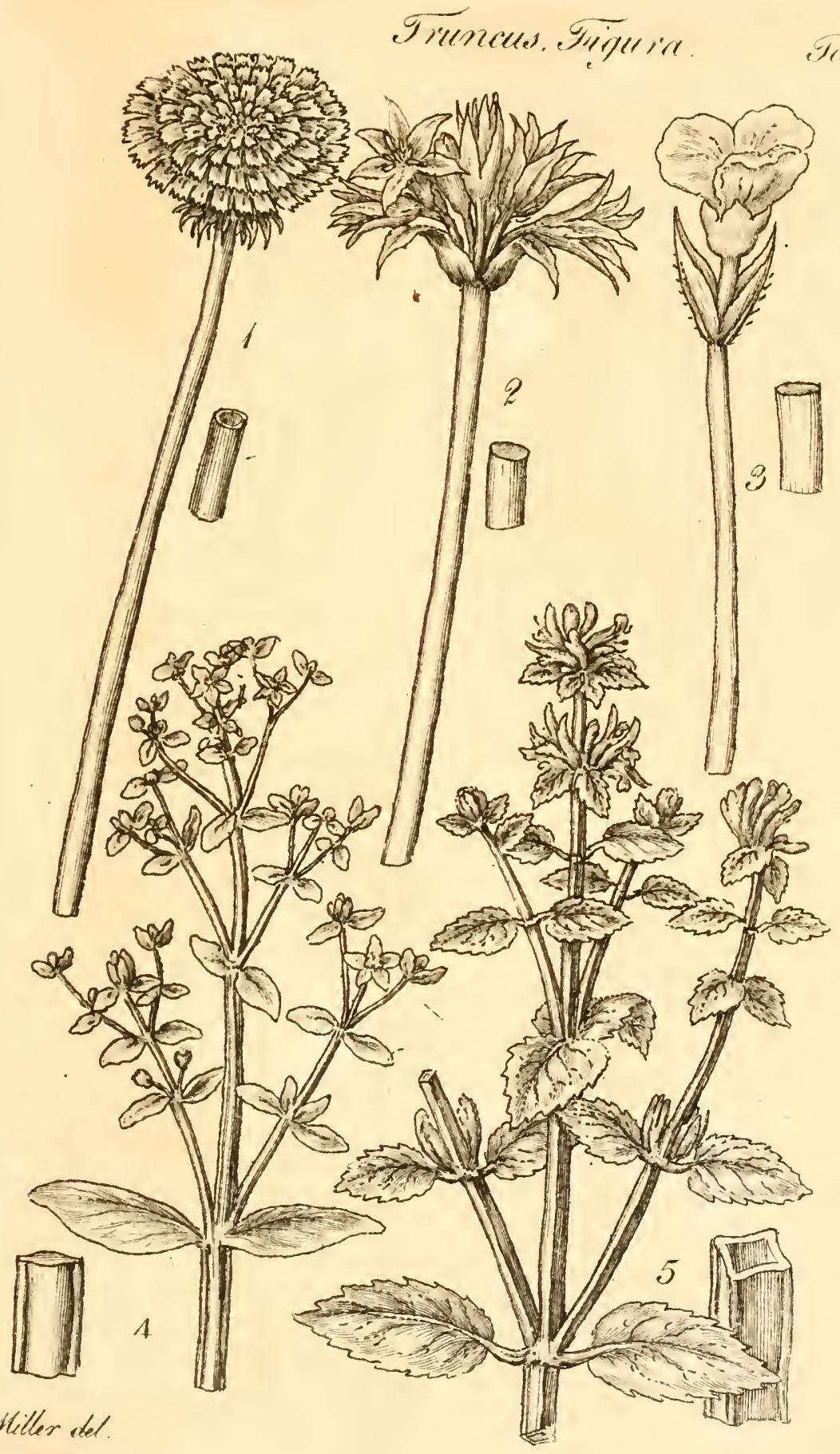





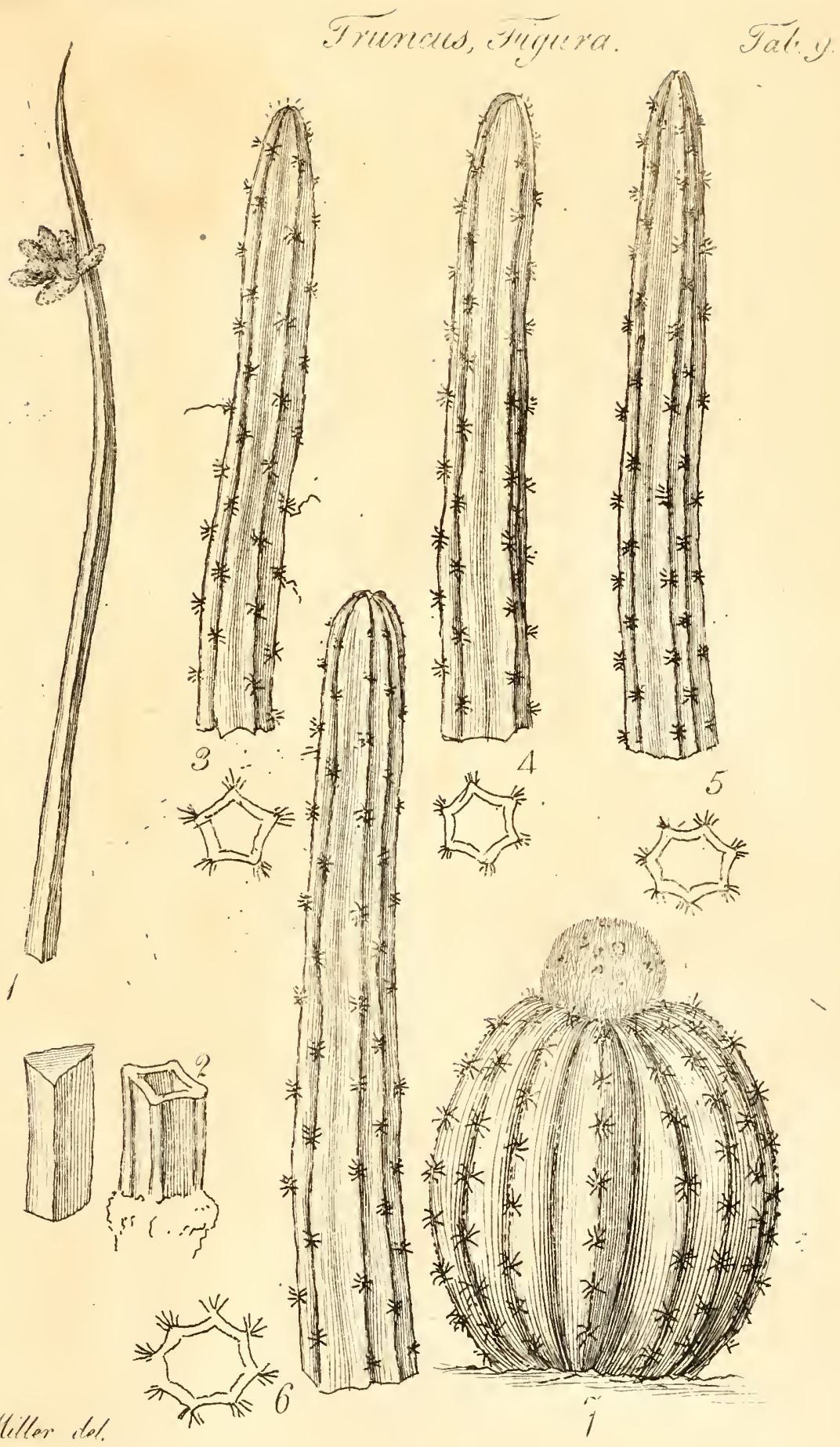



Grencend Testite.

Tat: Io.

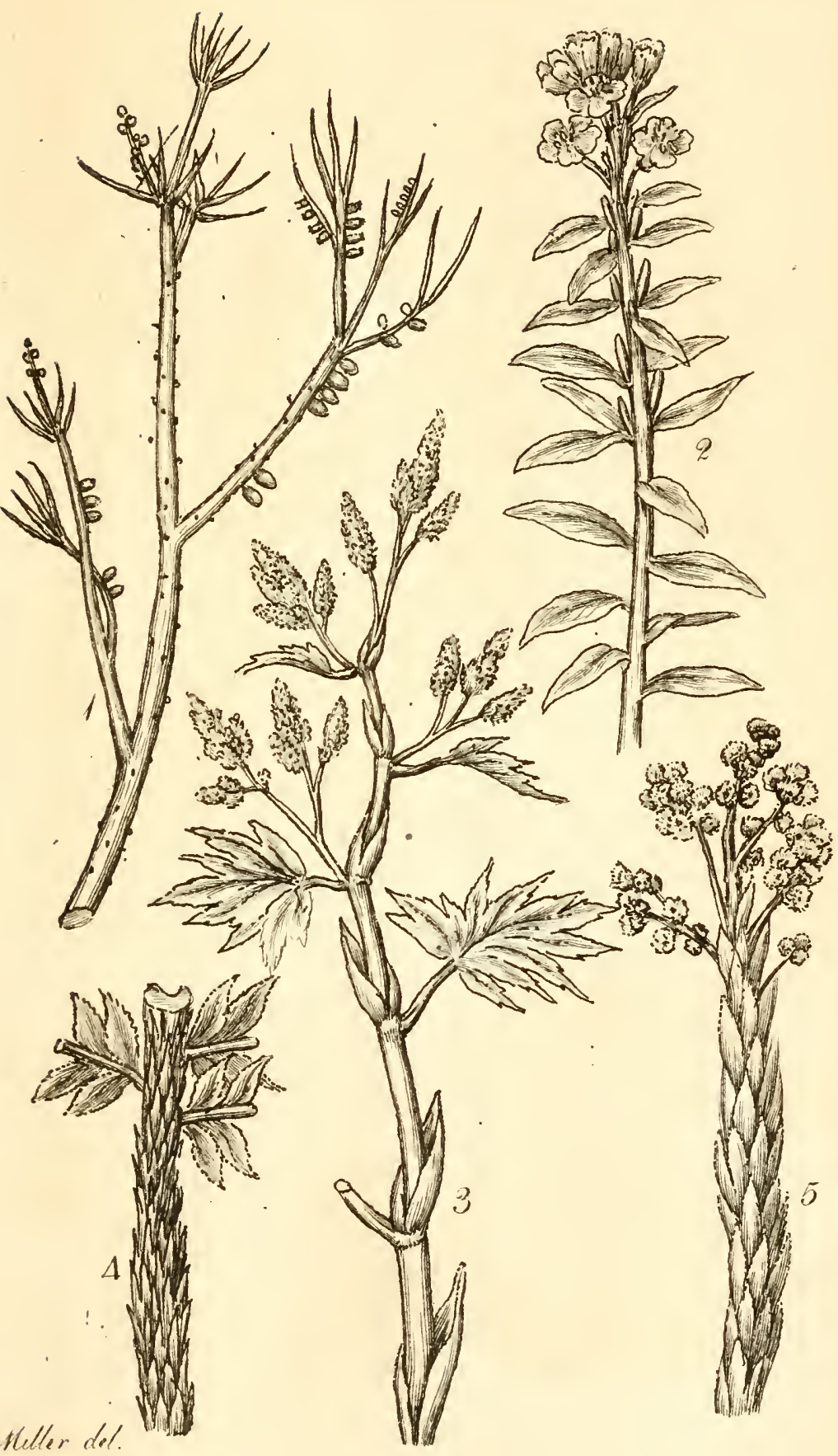





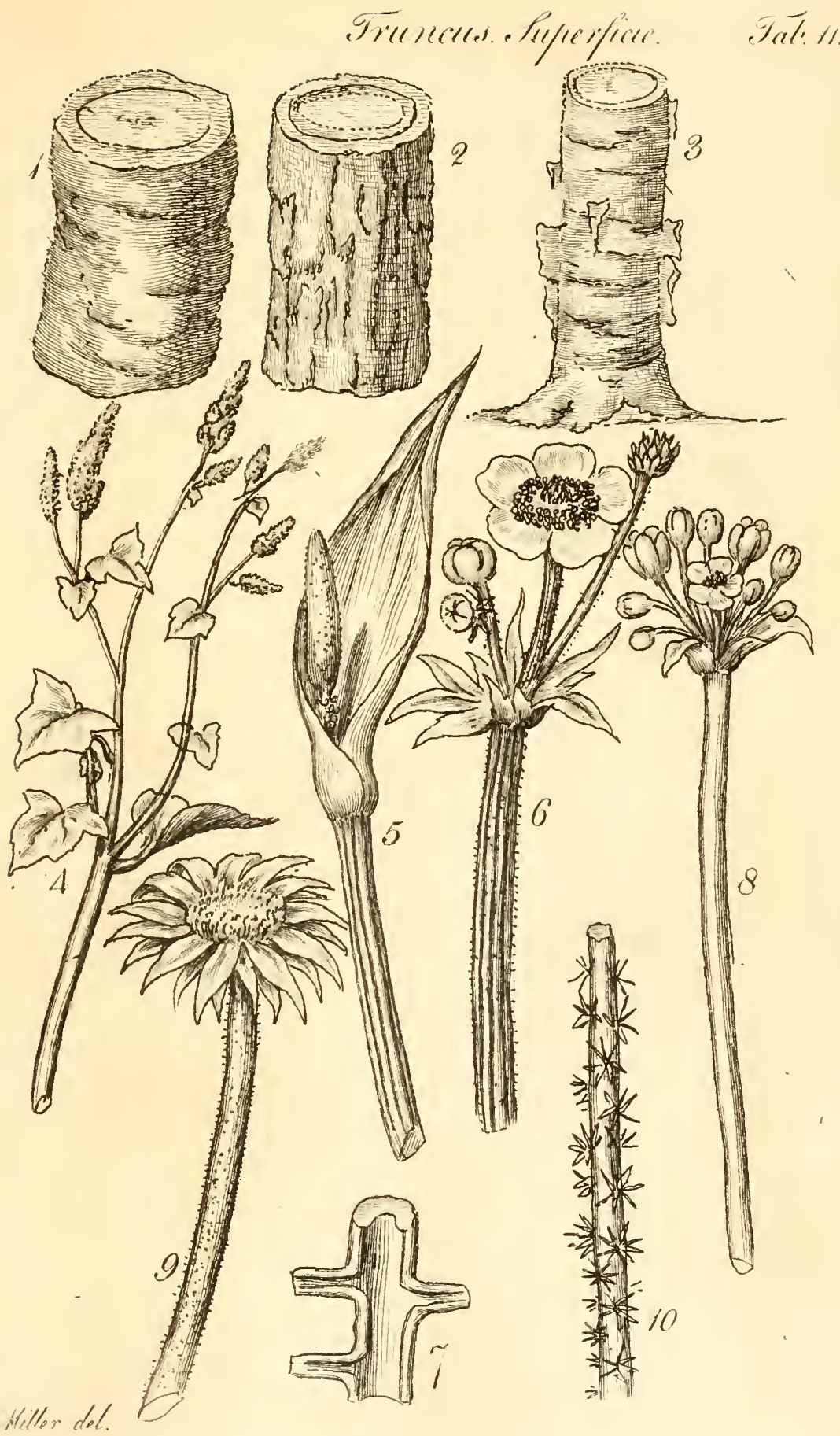





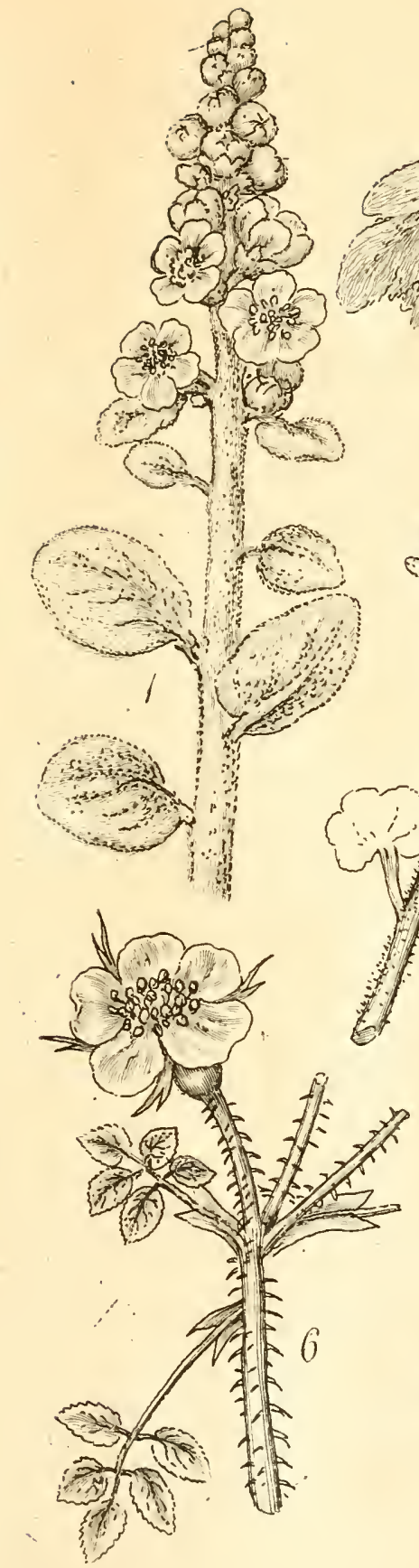

Grunces Shererficie.

yat. 12

6)

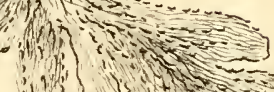

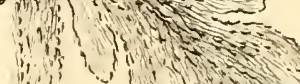

? 4 thity
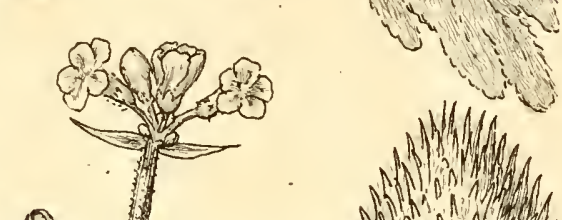

w) Whyde Minionstom Wh n n No 01390
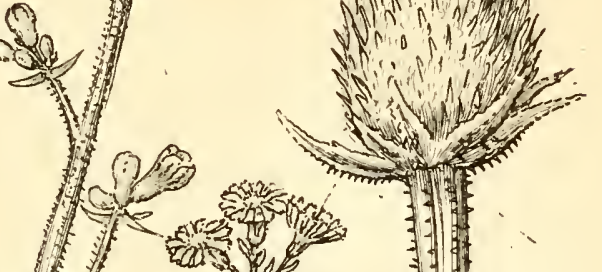



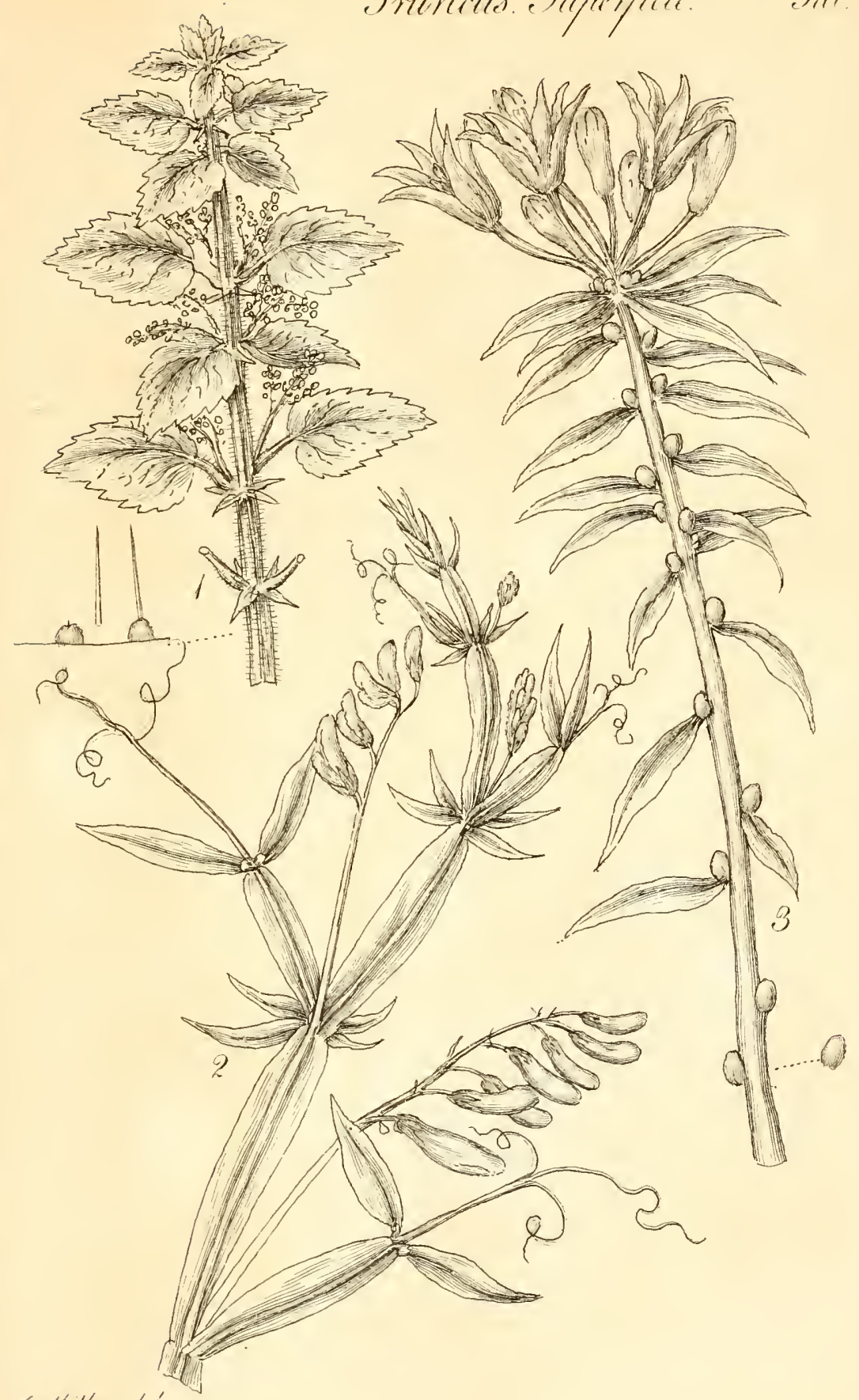

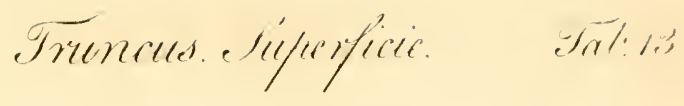





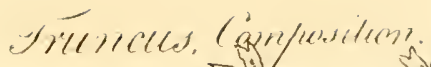

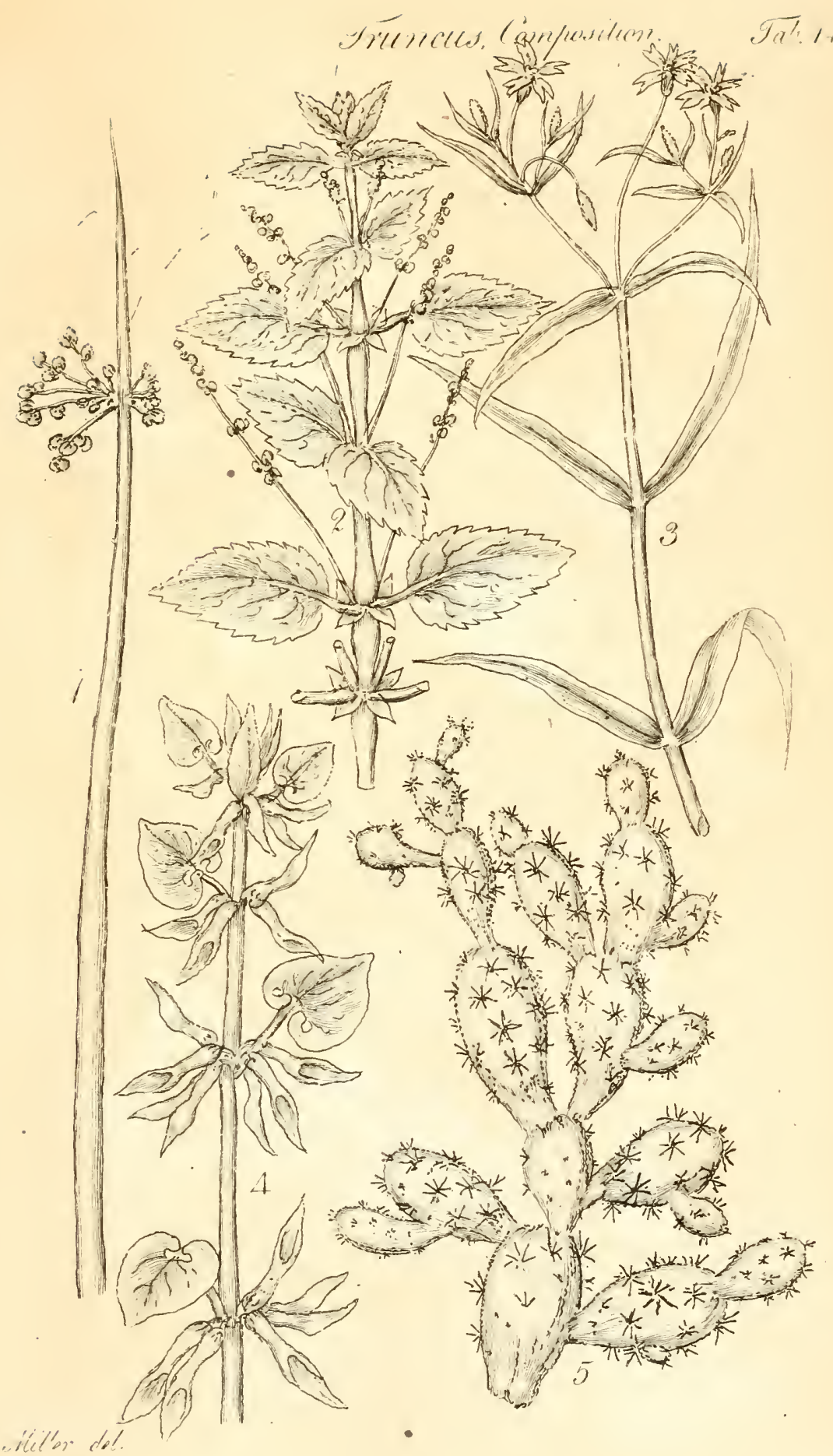





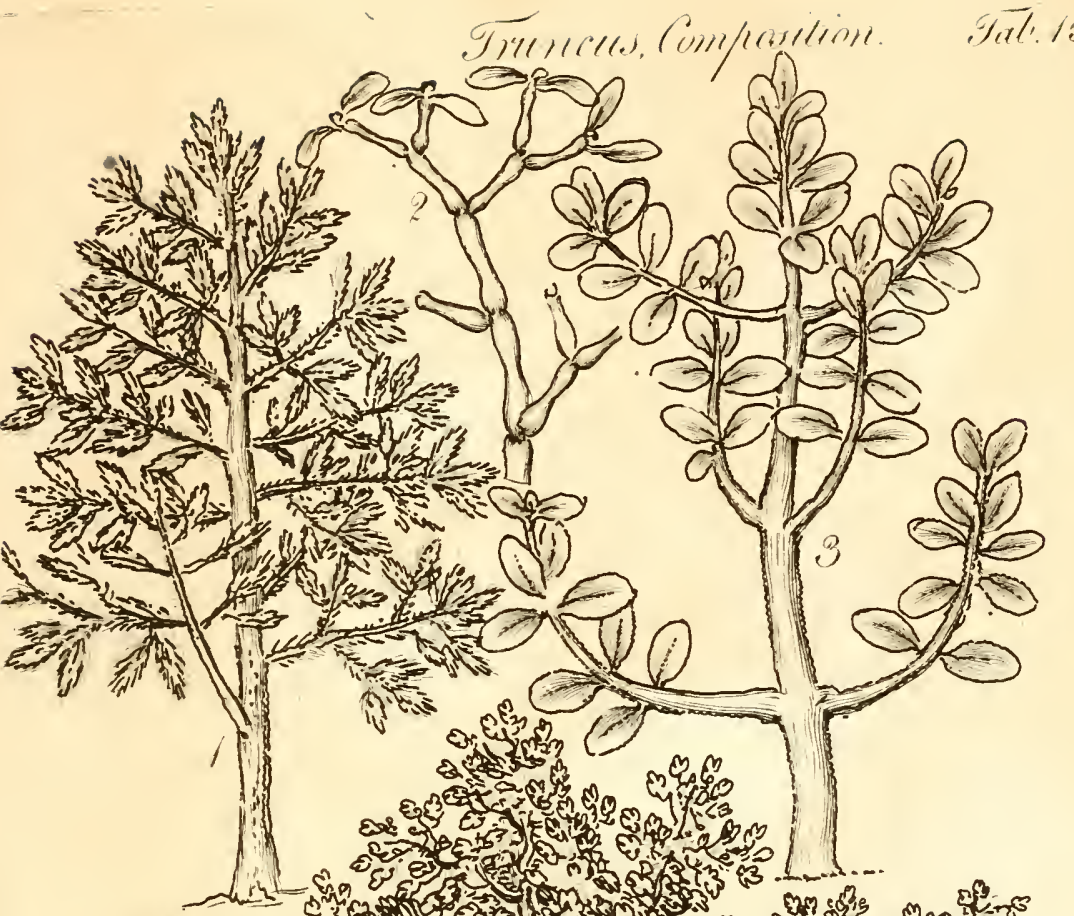

(3) a

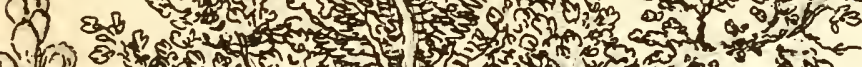

(1.)

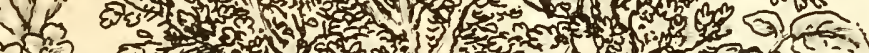
कर 63 घु R.

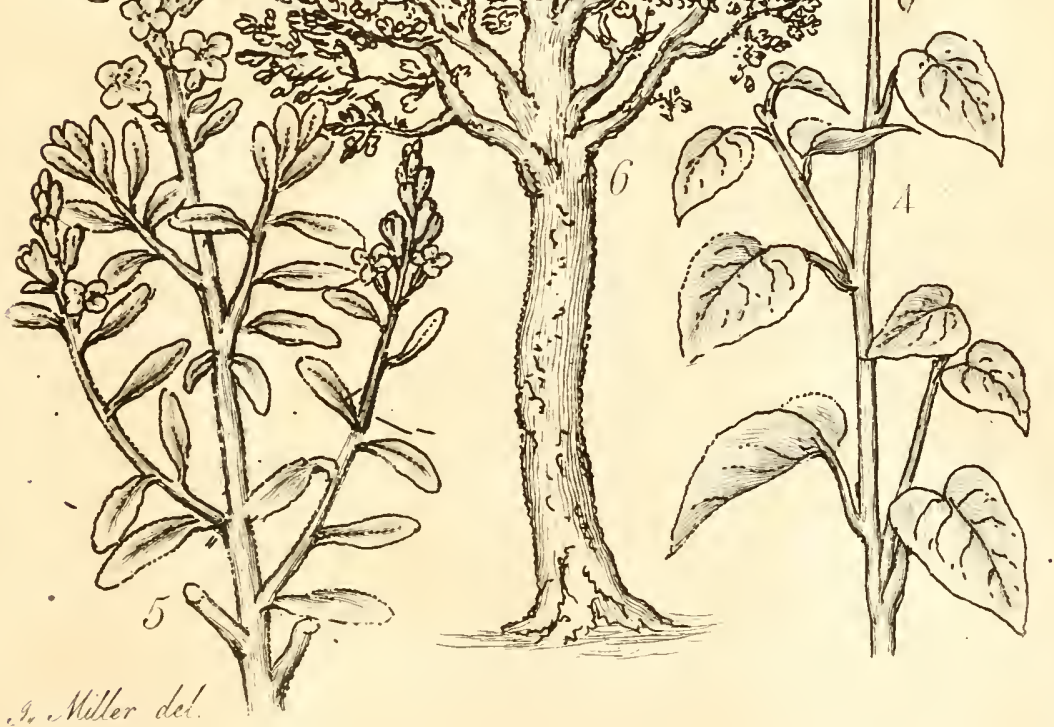





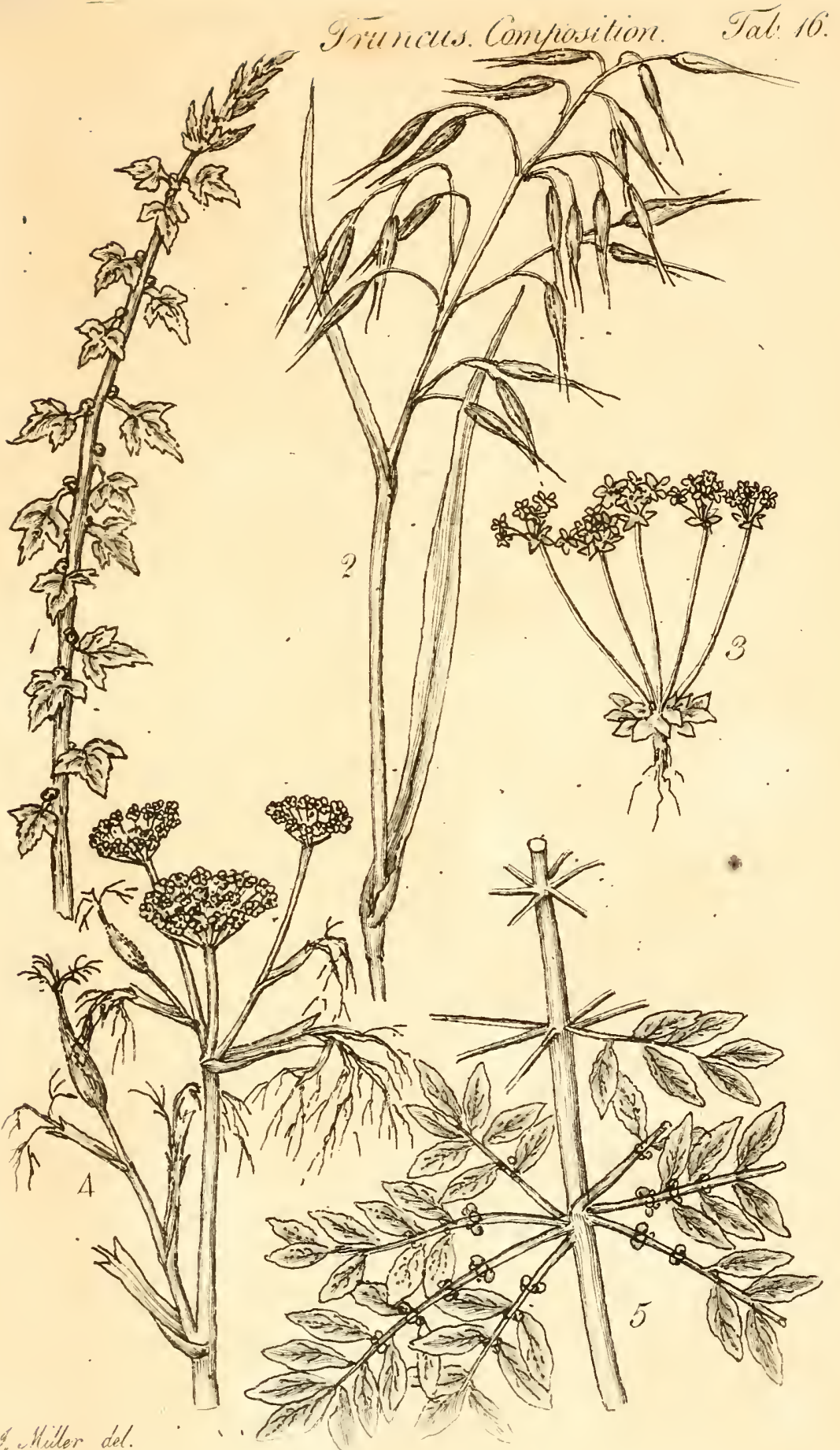





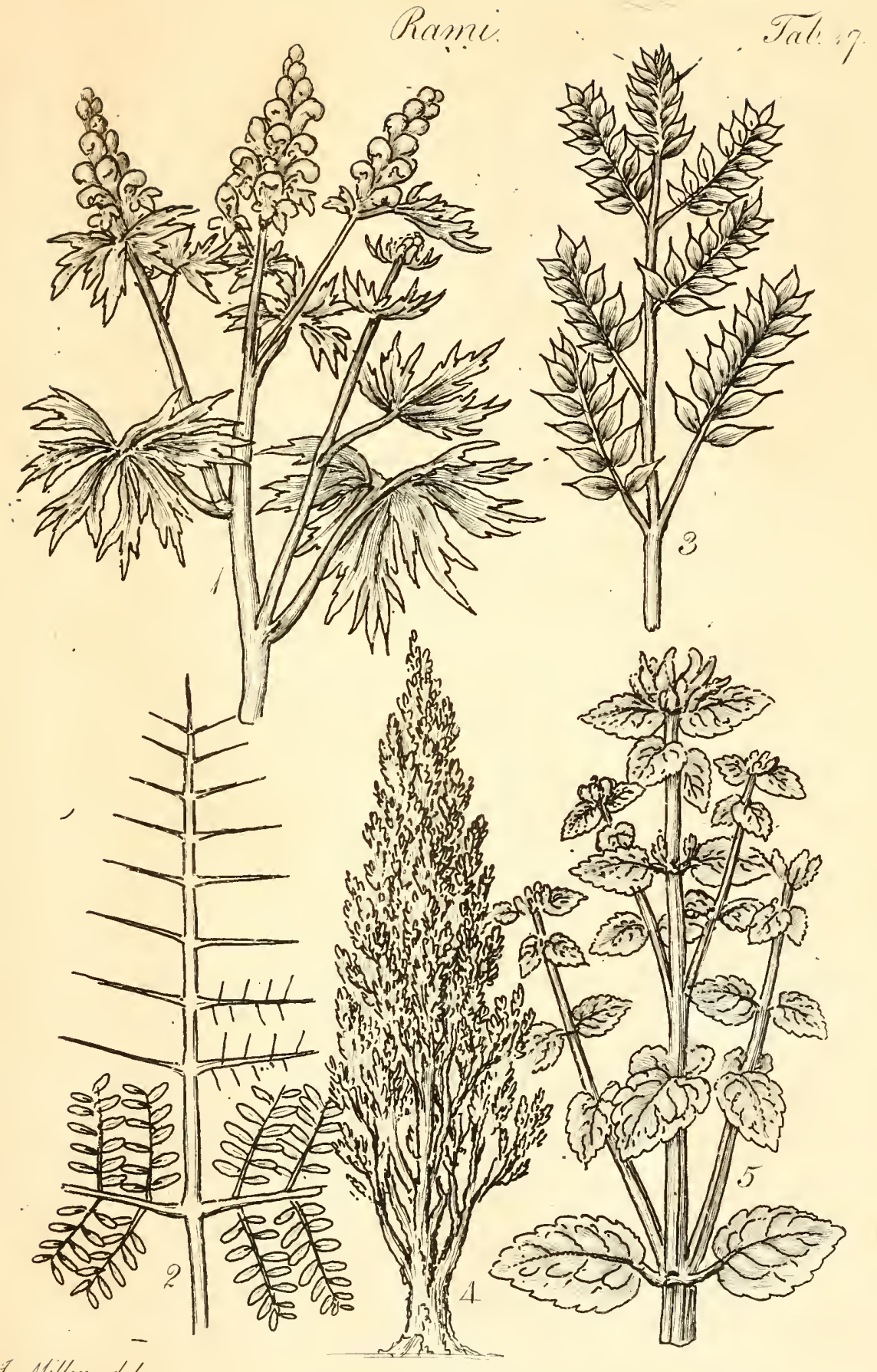



Rame

Fat: 18

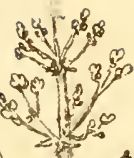

(a)

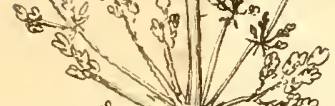

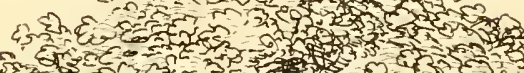

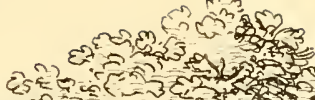

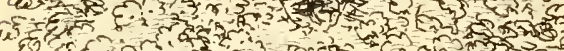

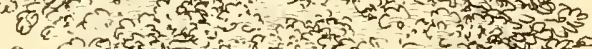

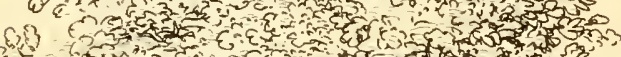

wan 5i धु के है। दर (1) 로영

(in) 13

到

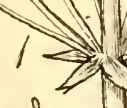

\% (1) $p(1)$ is Ecres of 10 (15 ( ) She 0 .

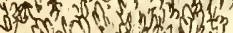
3015 L 20 ote D.

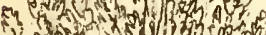

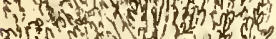

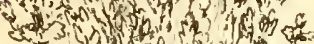
Rifi sis

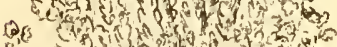
ine of

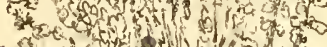

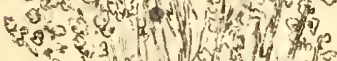

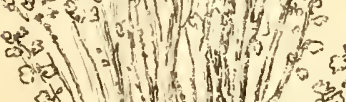

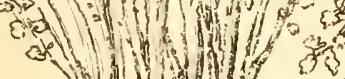
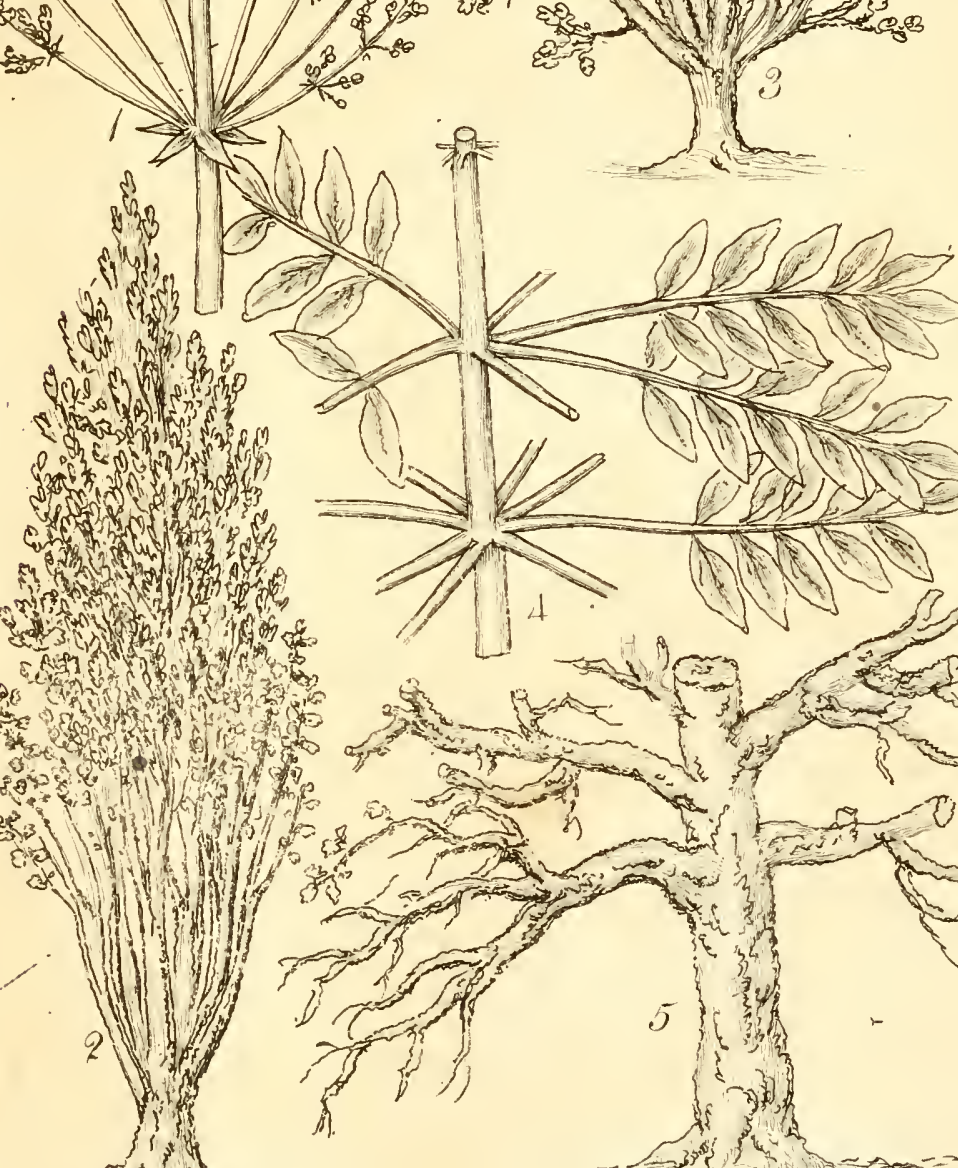



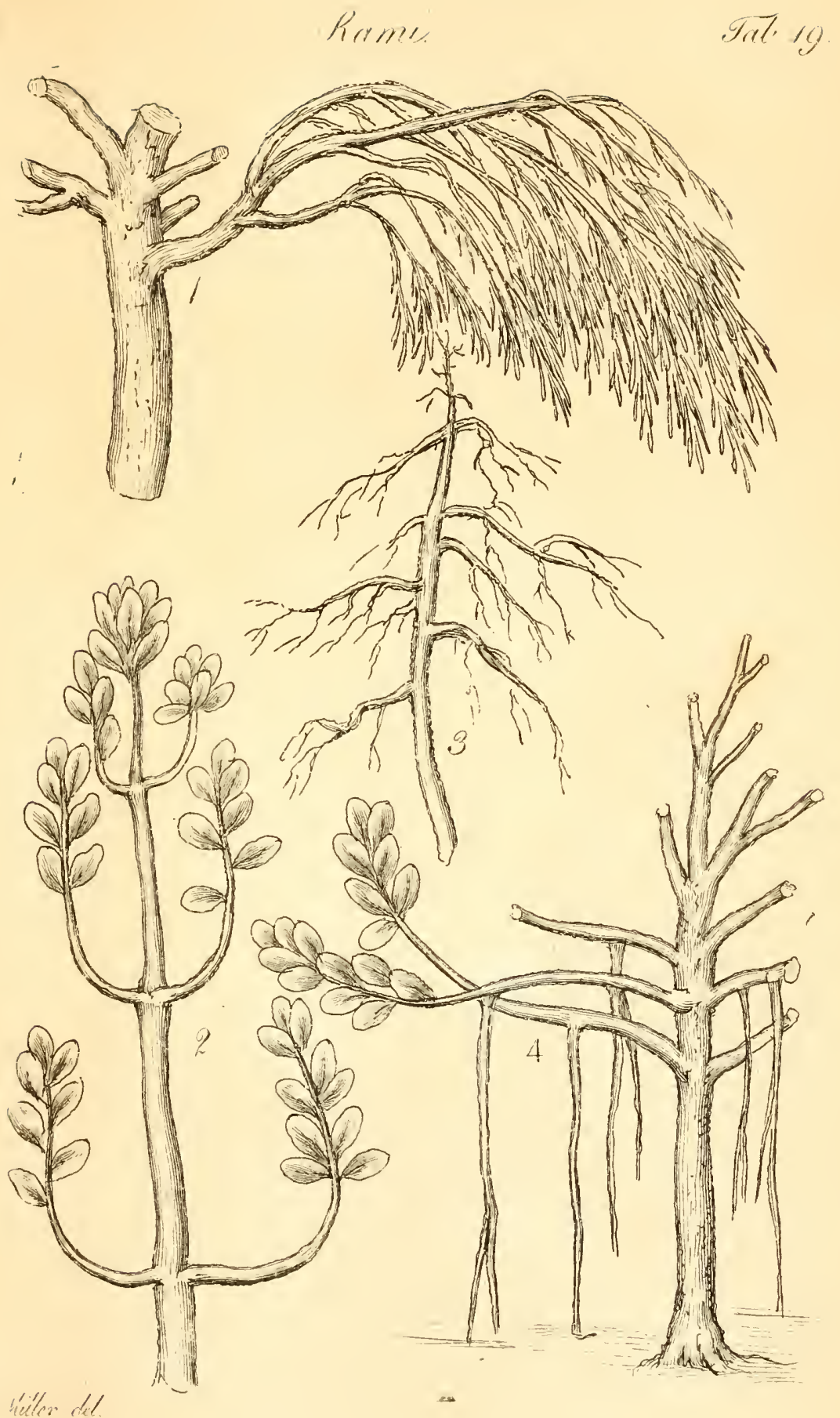



- Minigile Leaves.

Yut:?

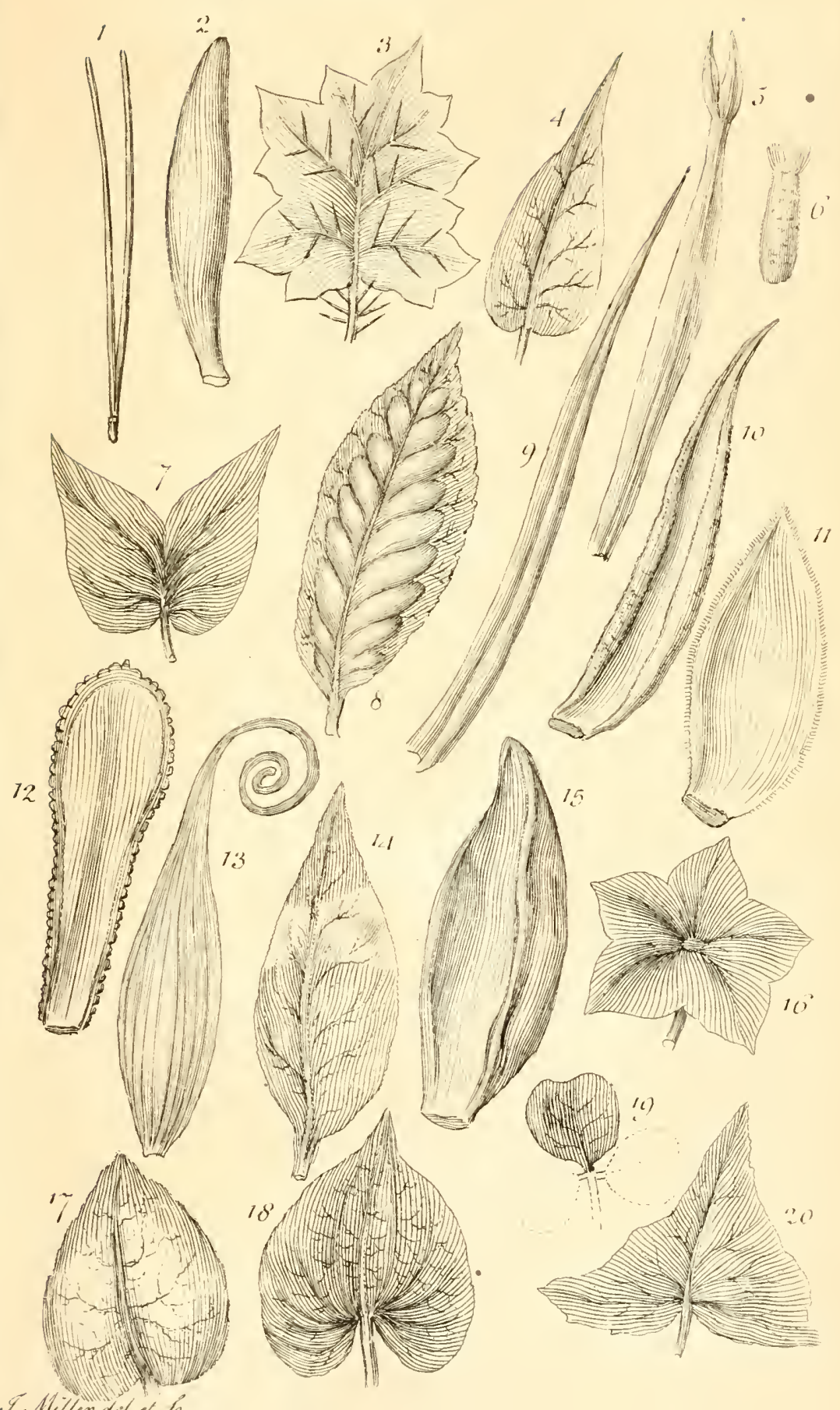











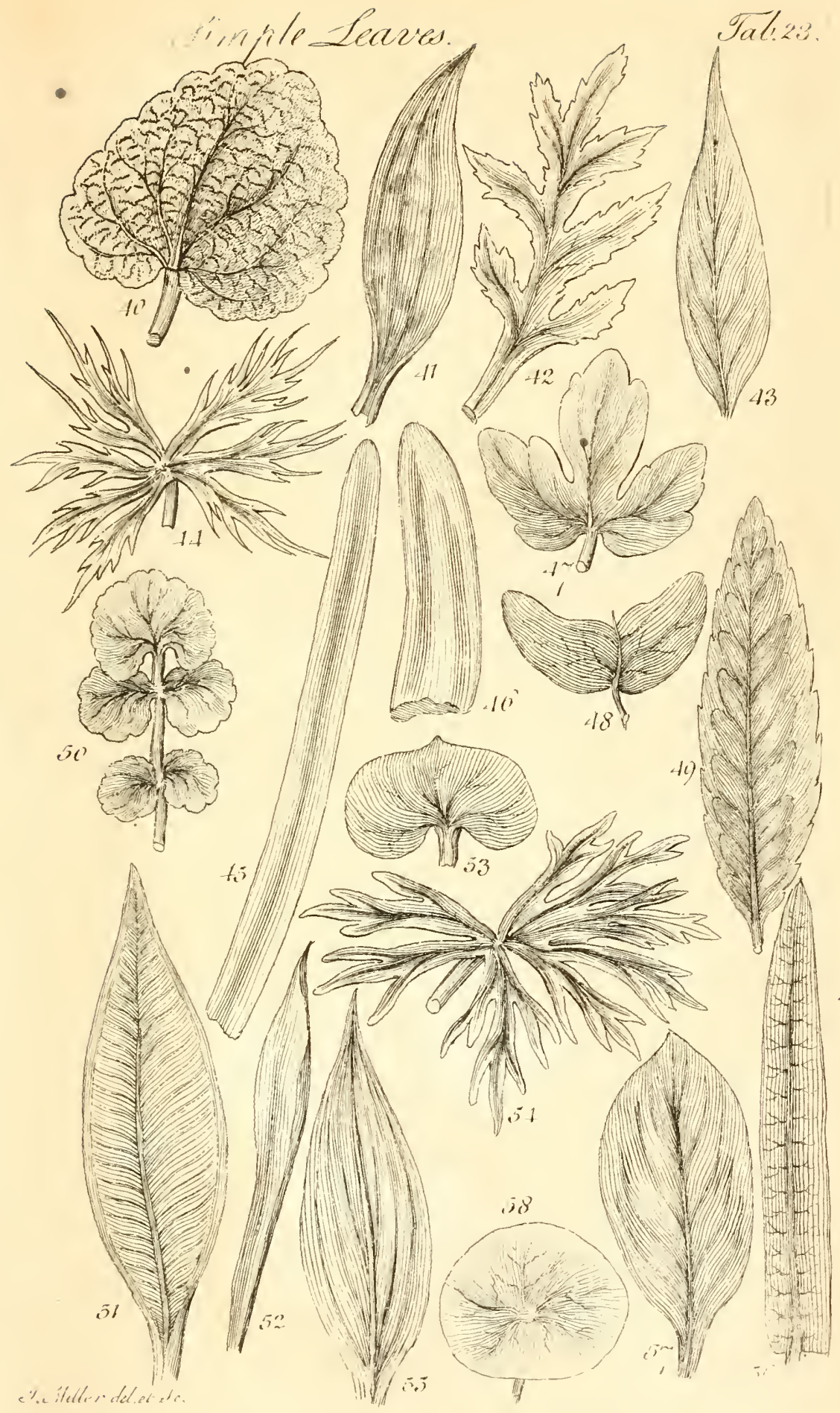





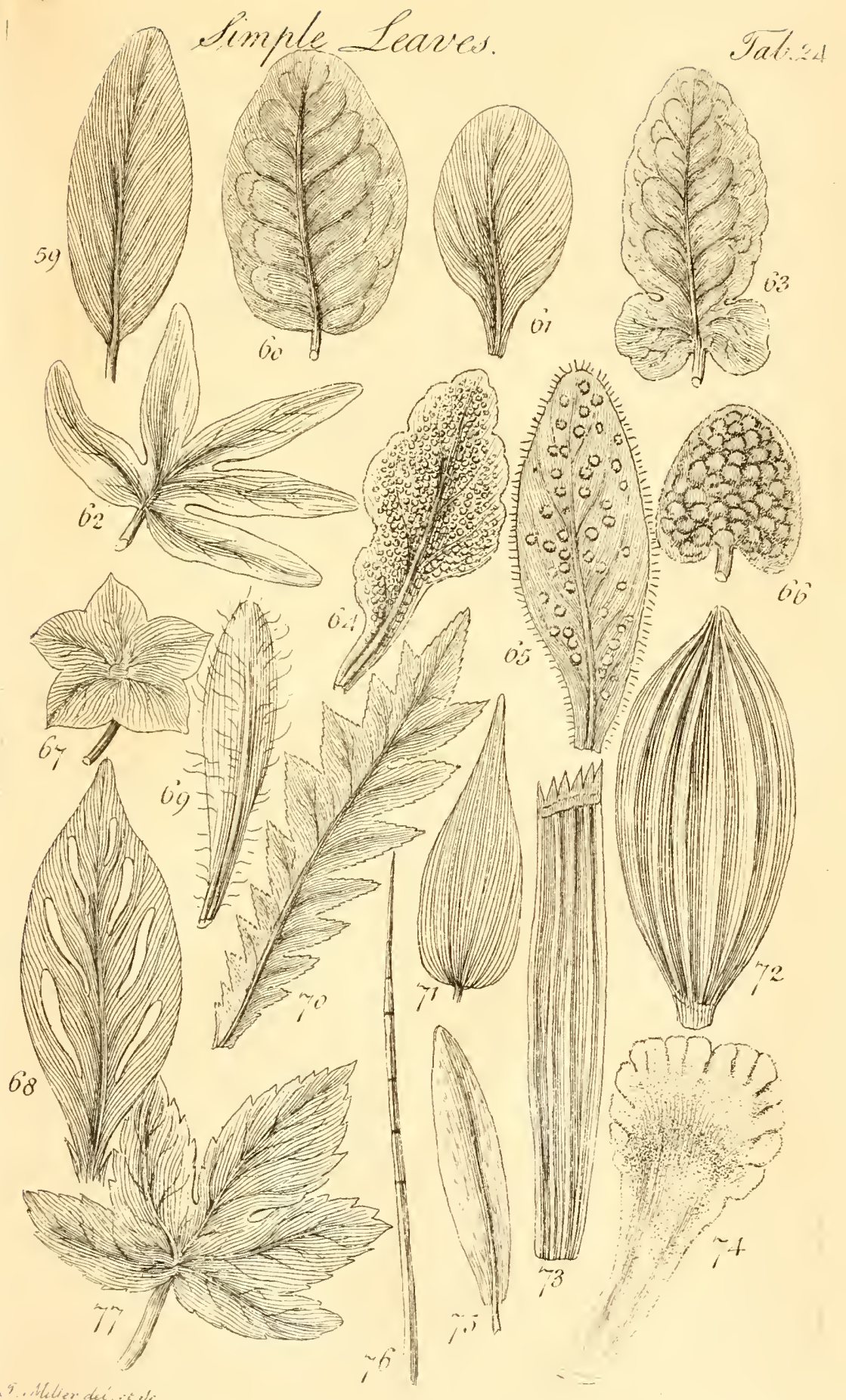





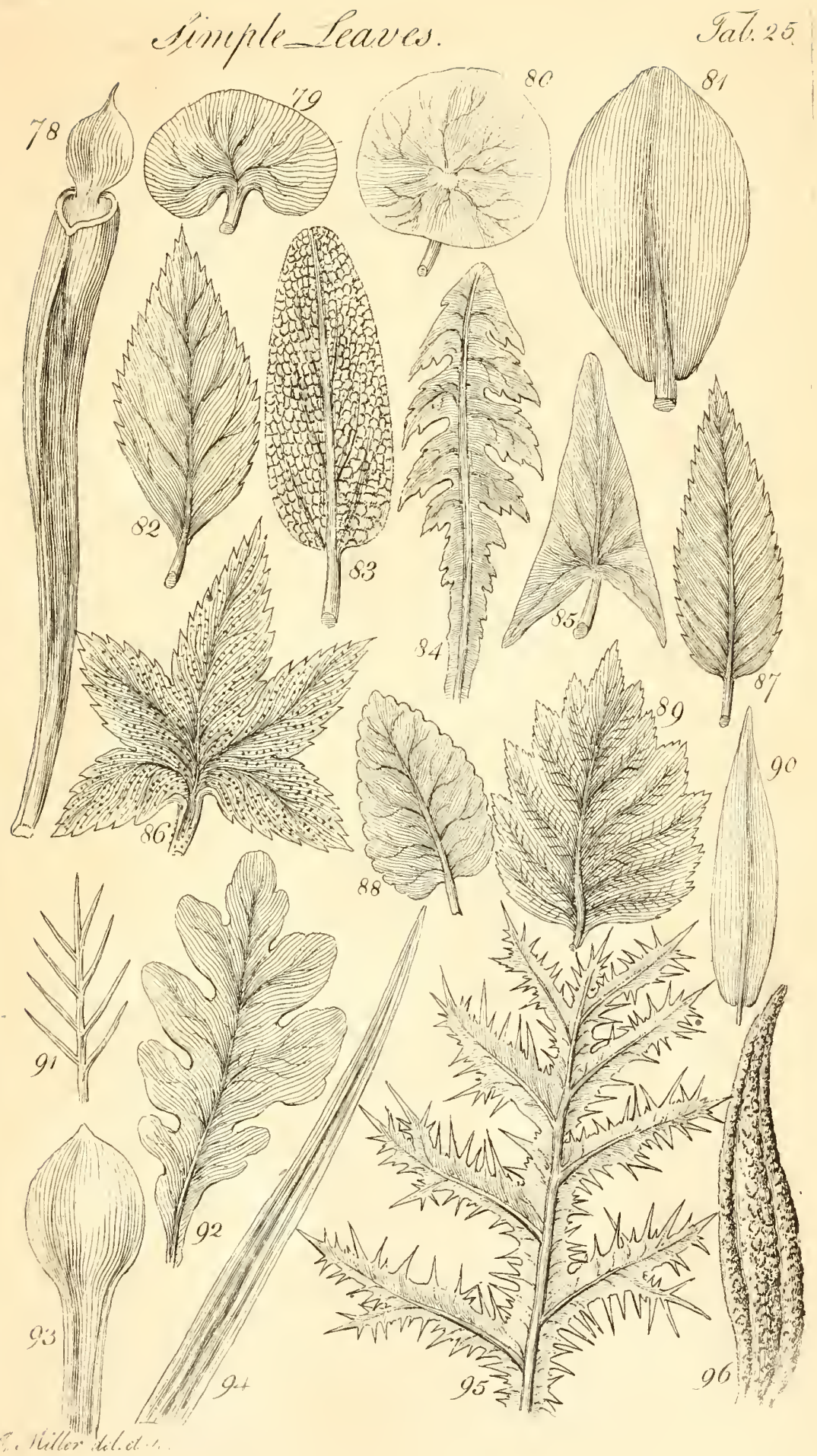





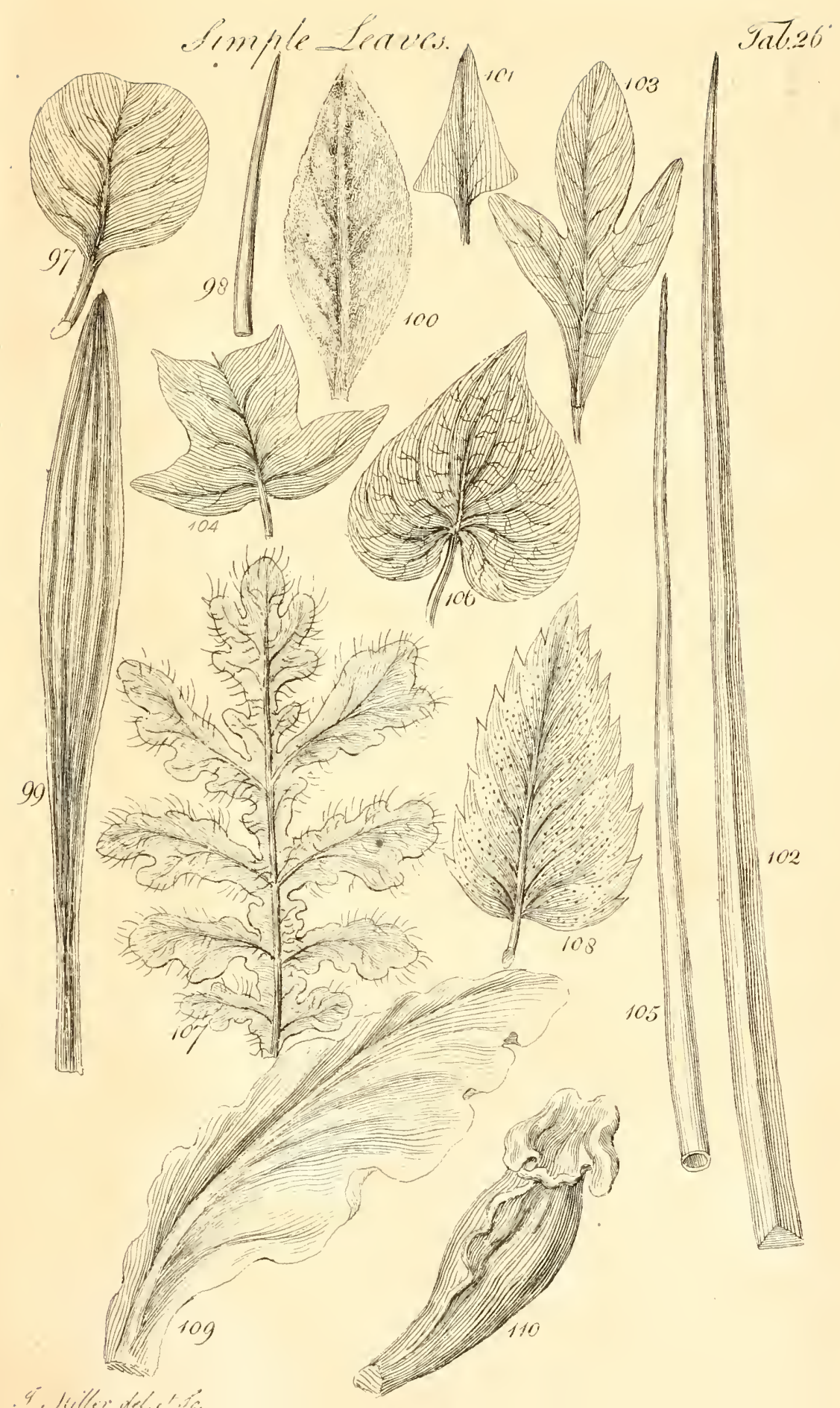




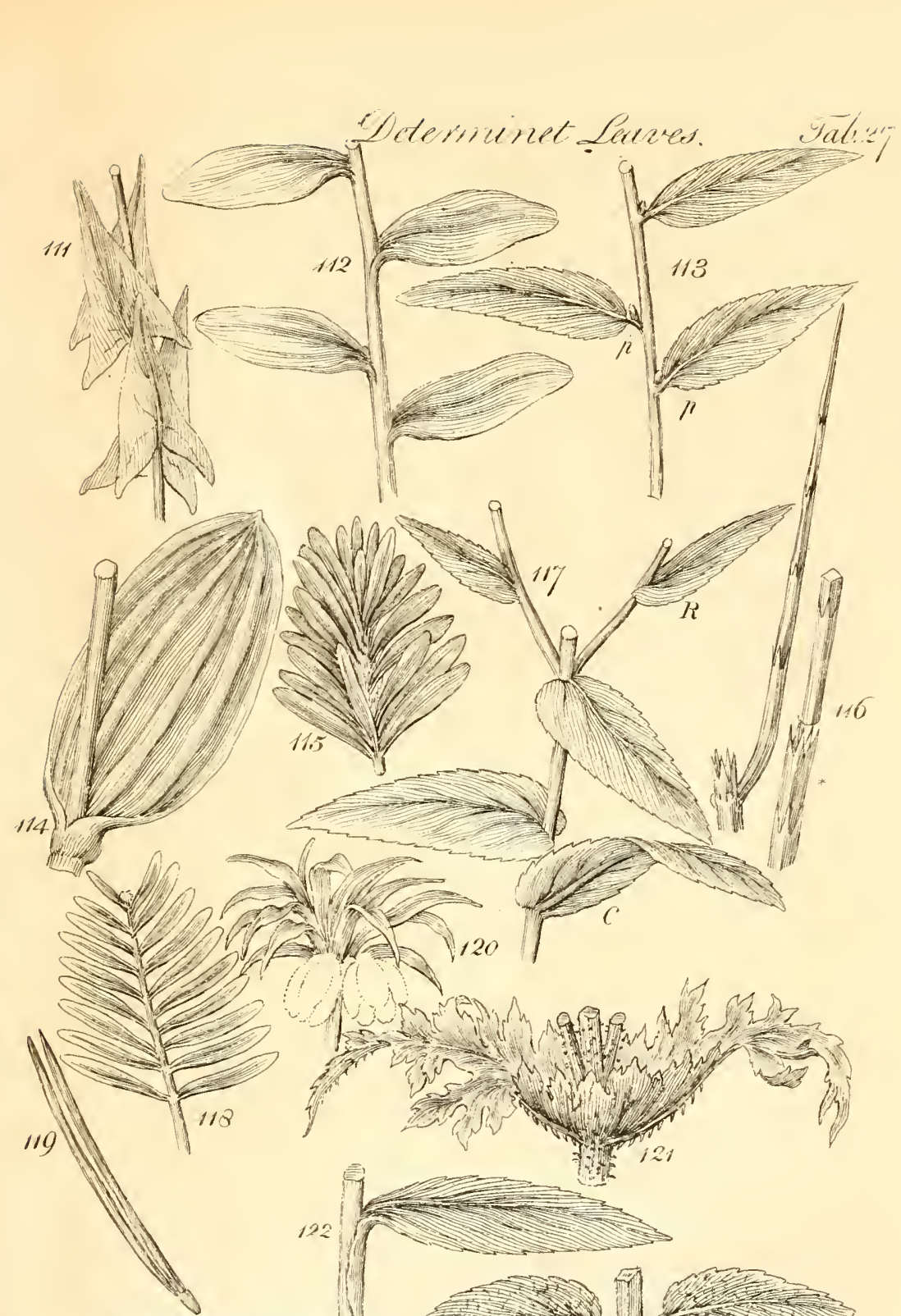



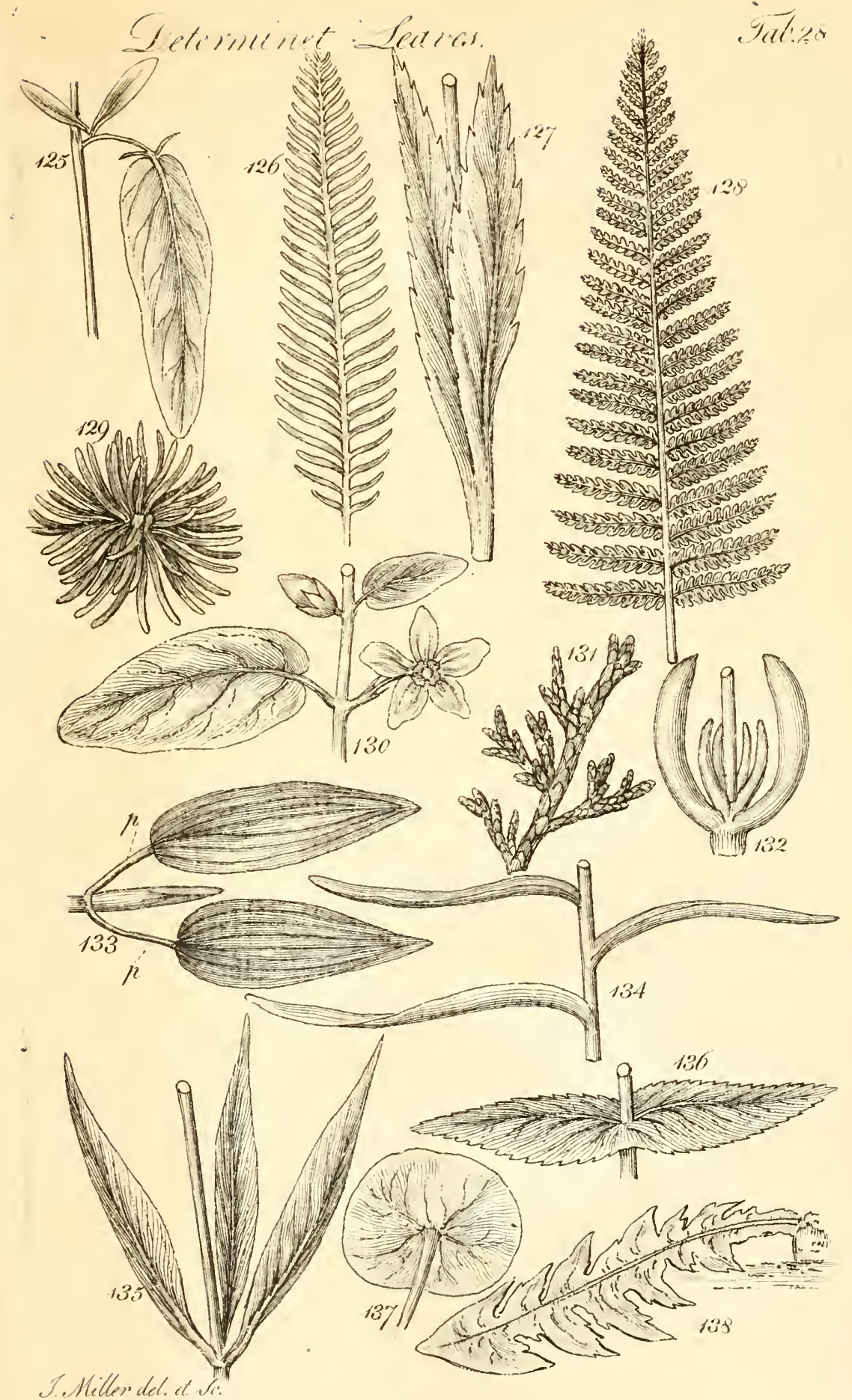



Leterminet Leares.

Gatiy
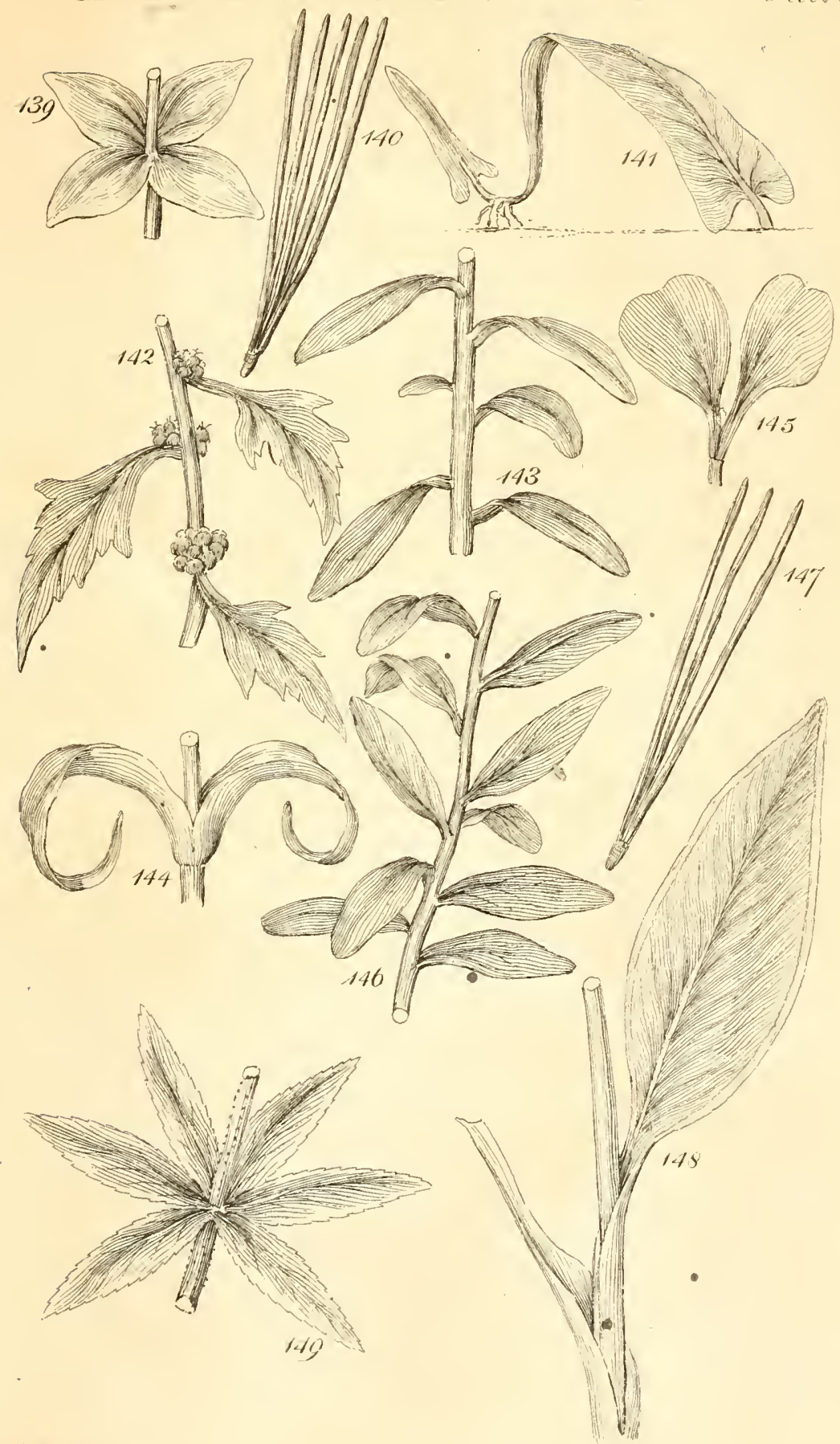



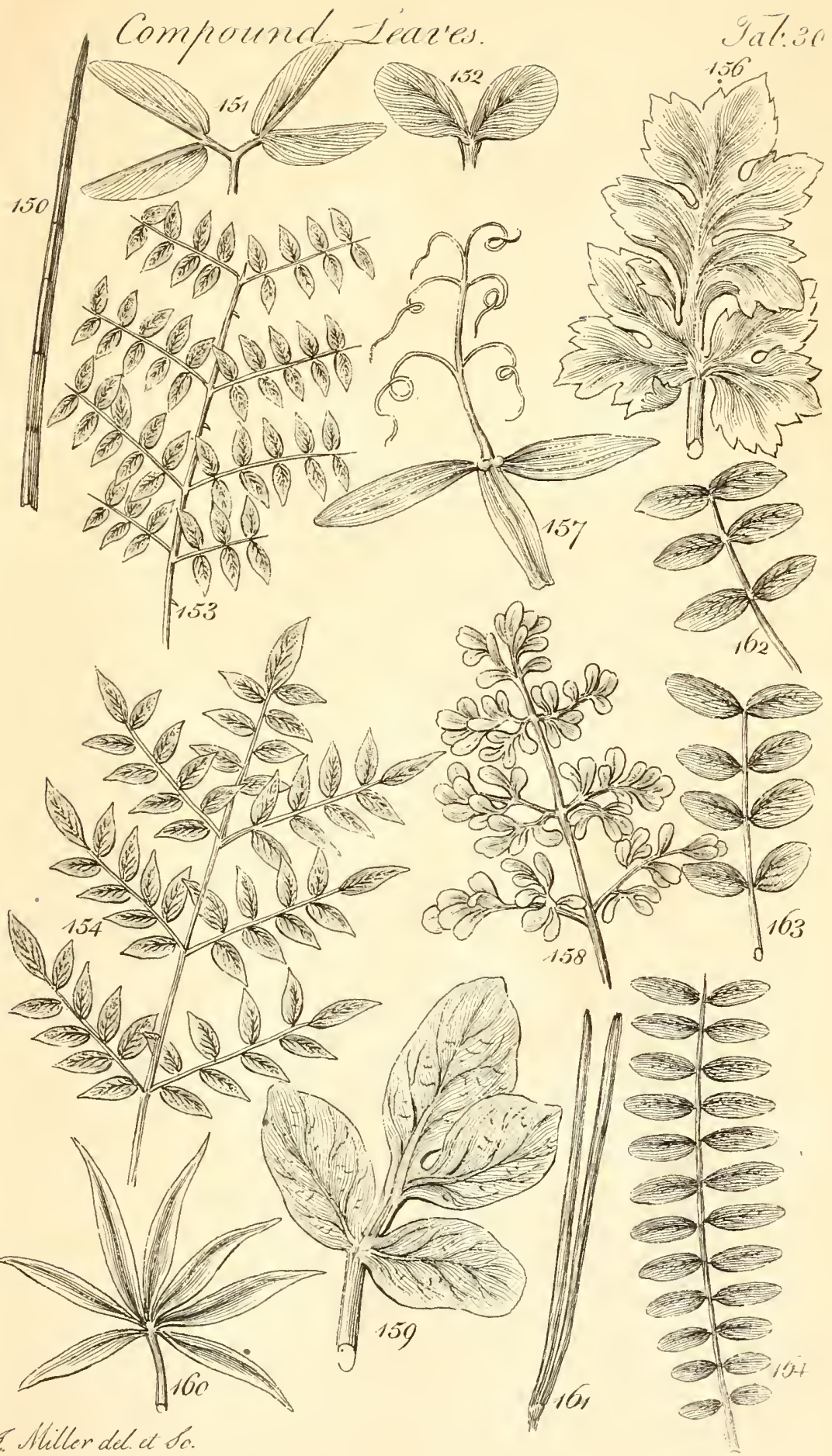





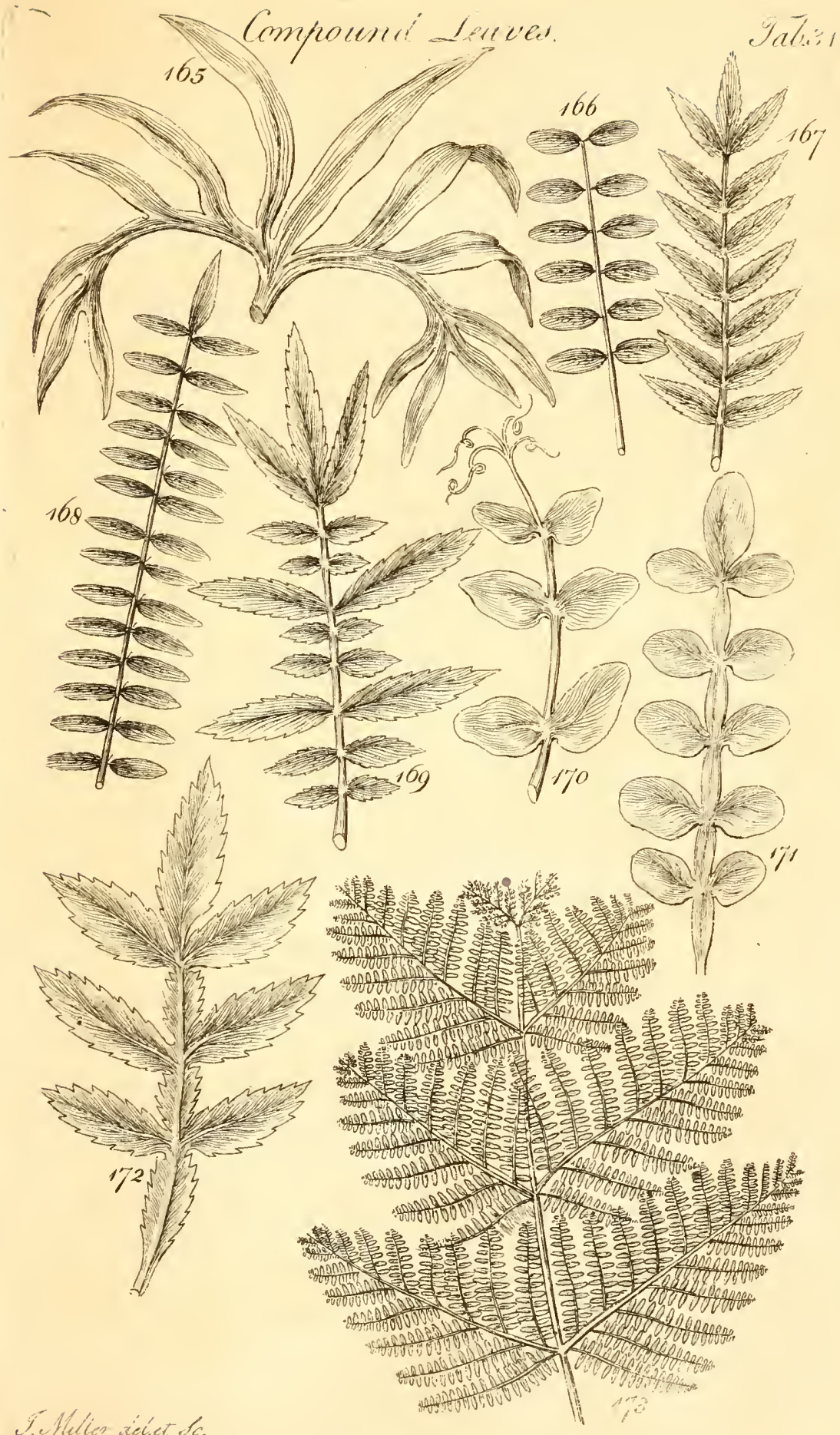





\section{Comprount Lérexs.}

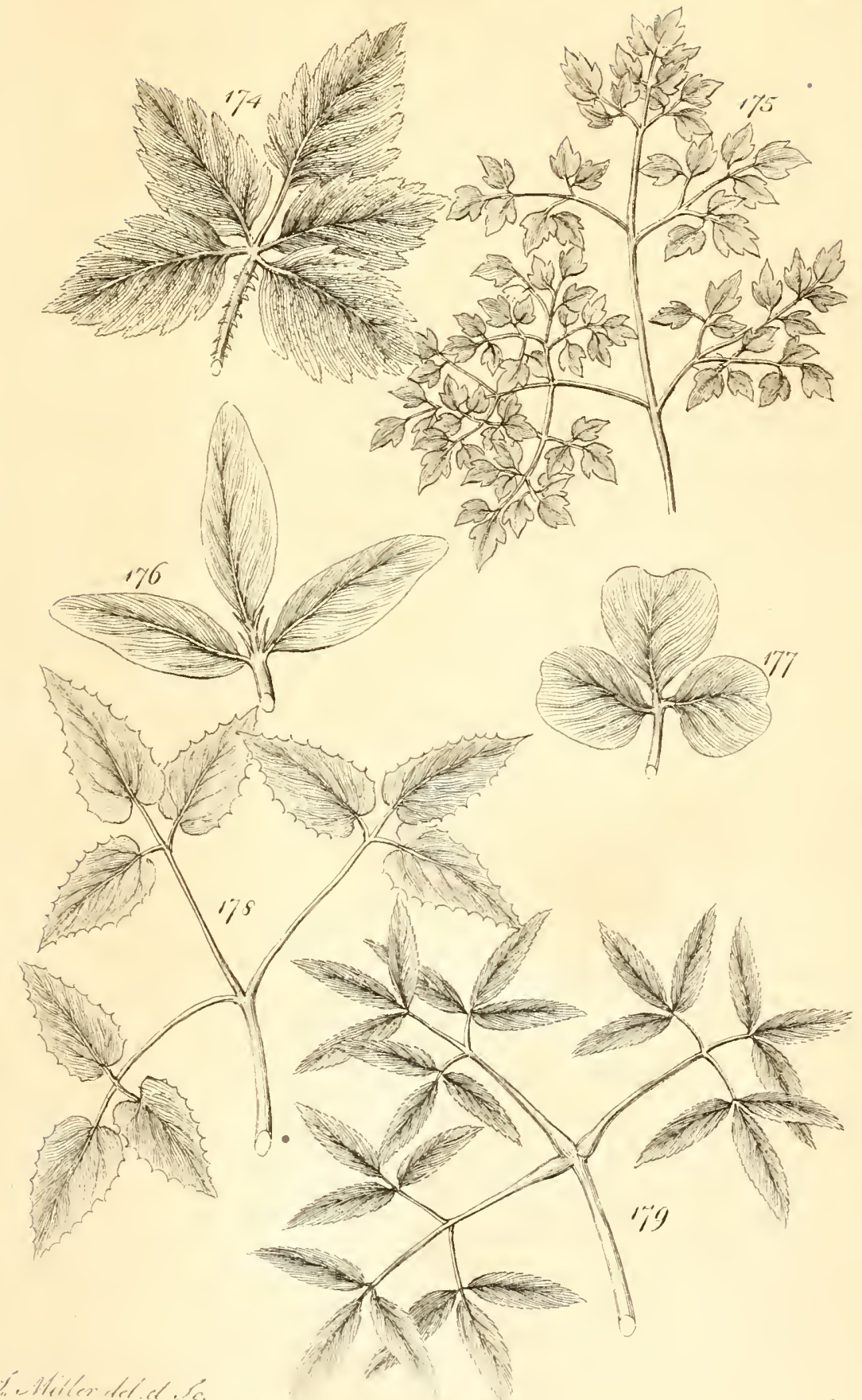





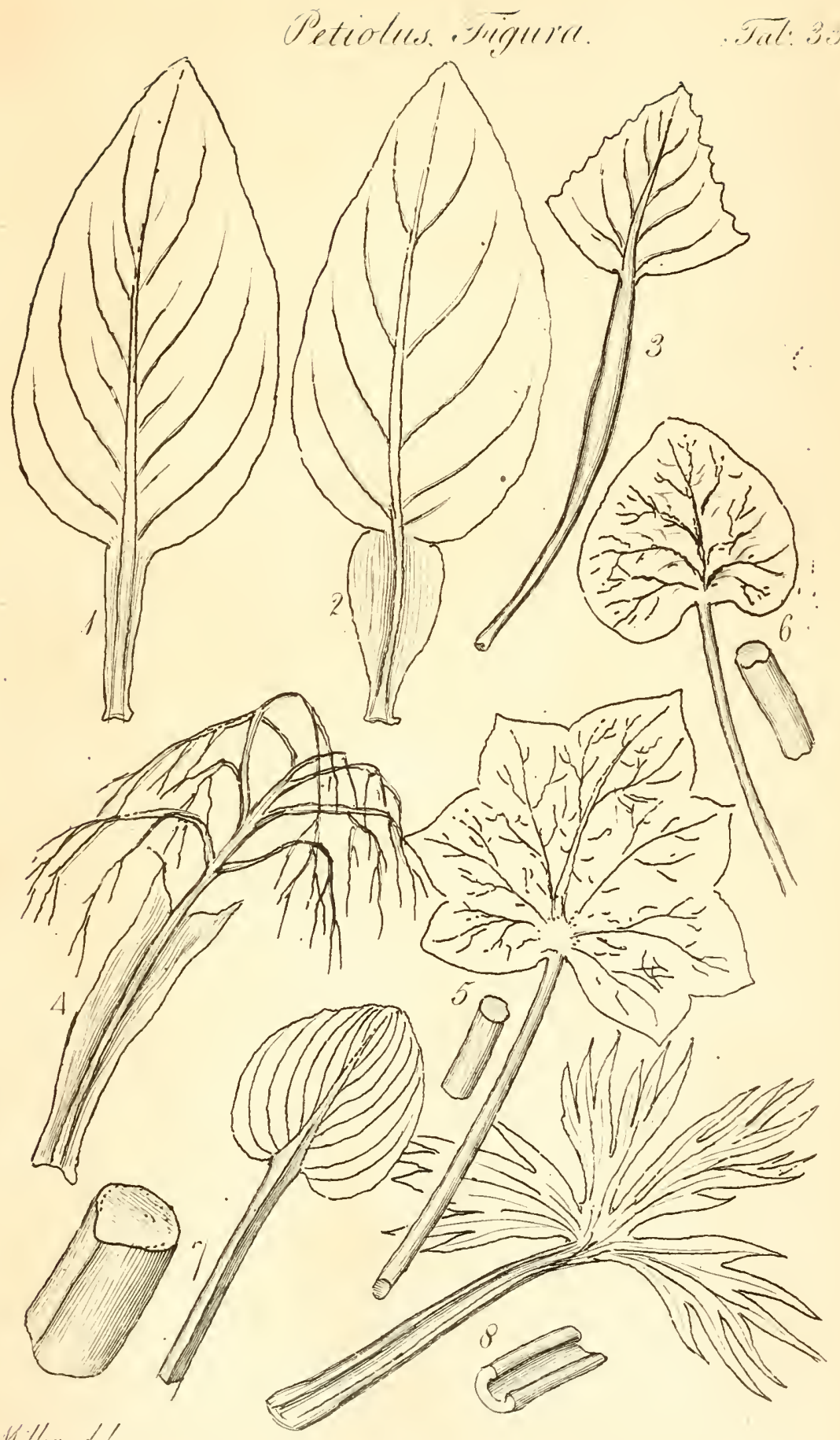

\%. Hiller det. 



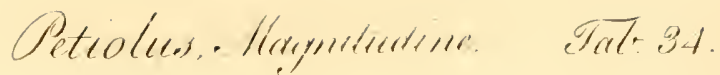

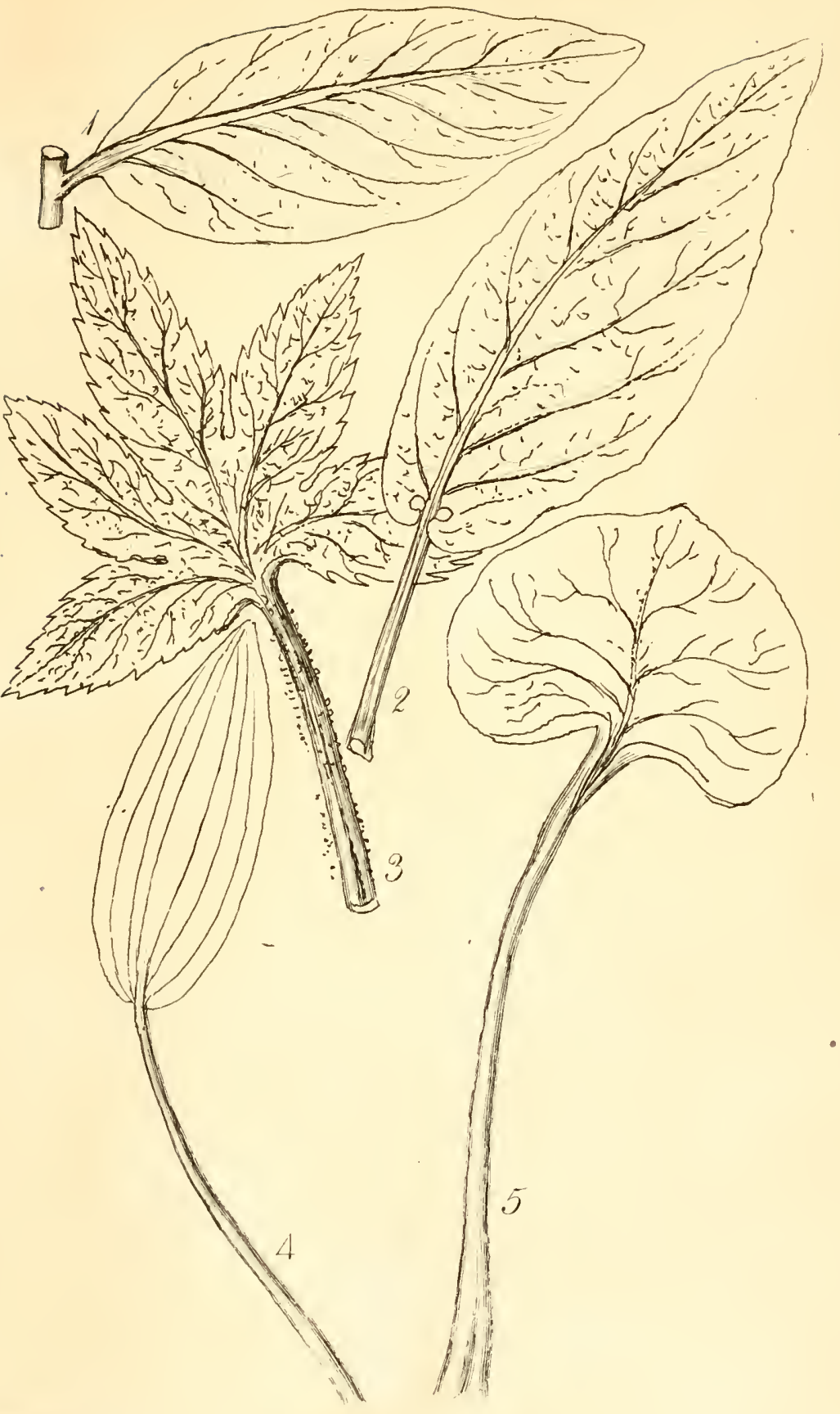

9. Stiller ded. 



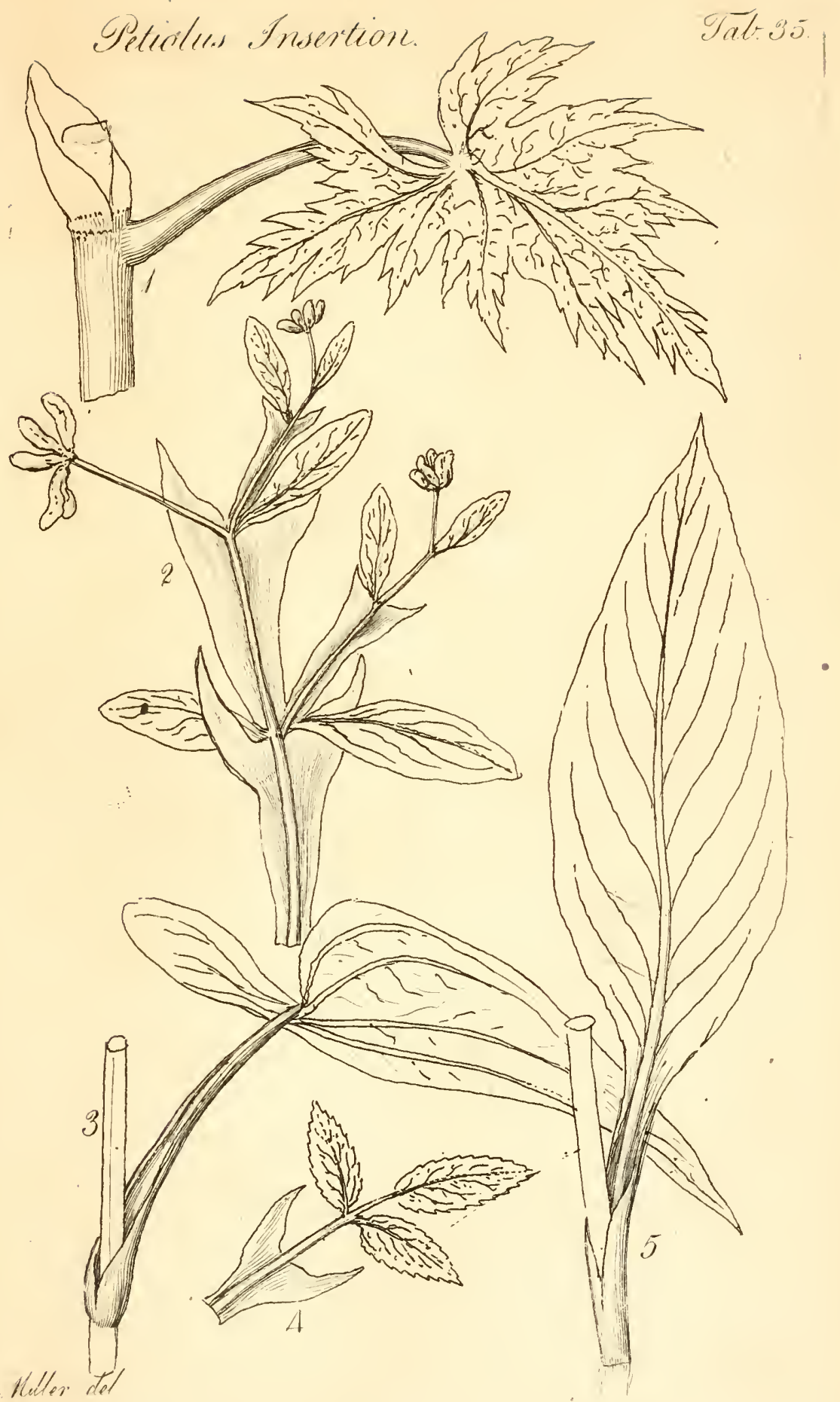



- Petider Divection.

जetrobs

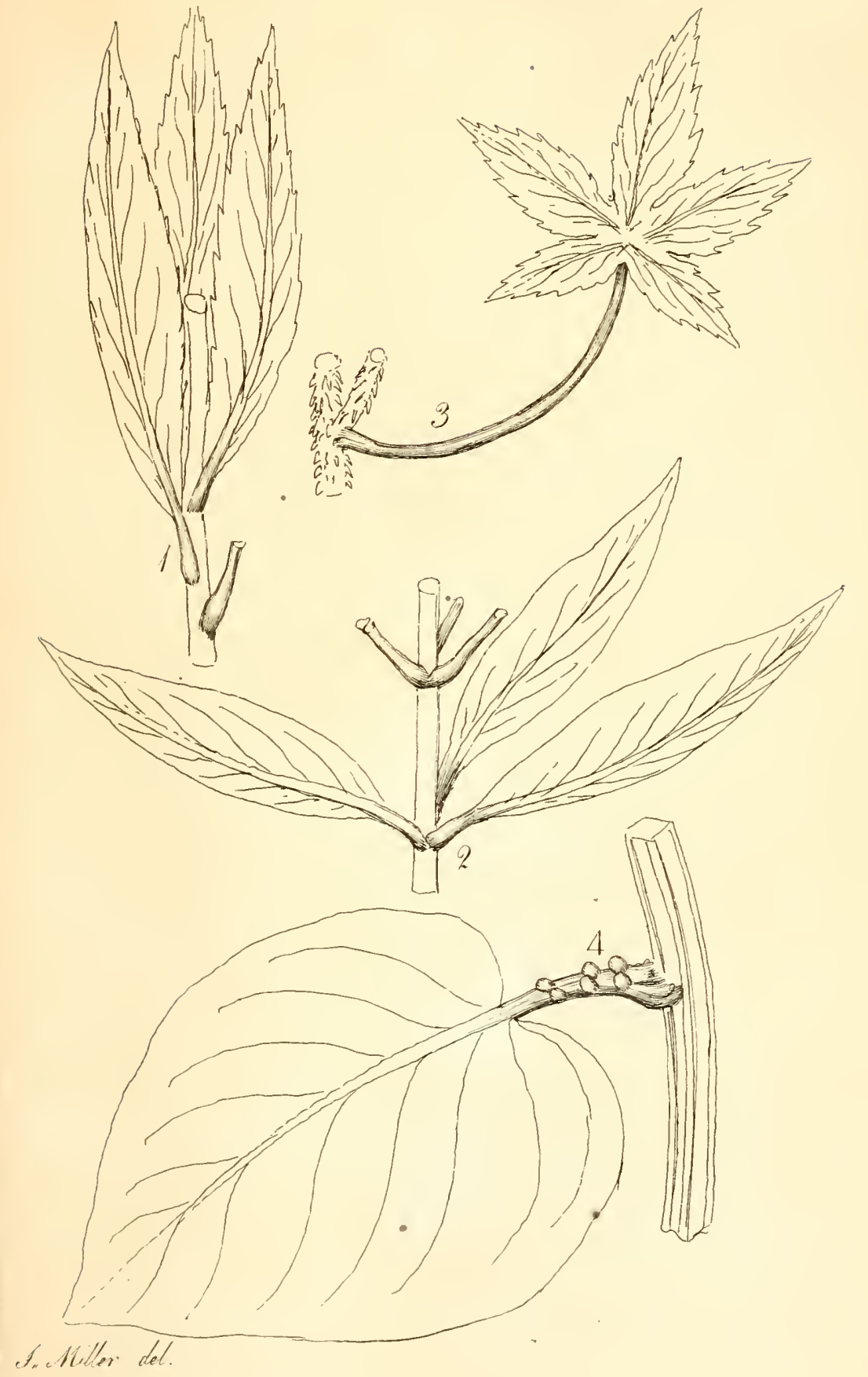



- Selentier éniperfice

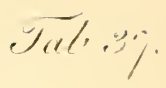

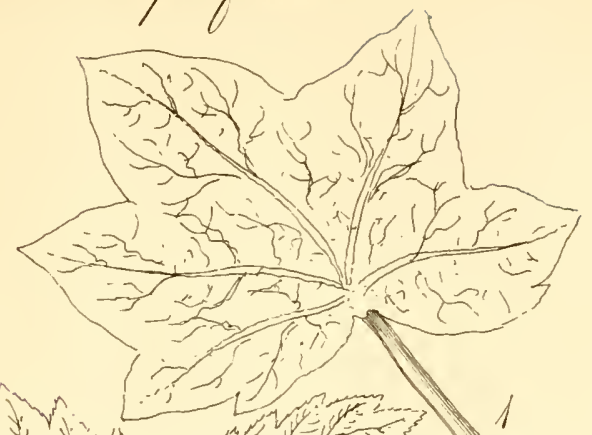

s.

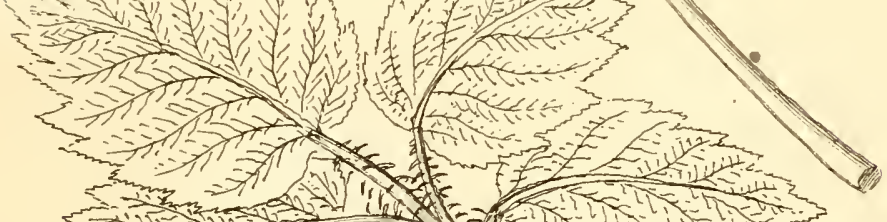

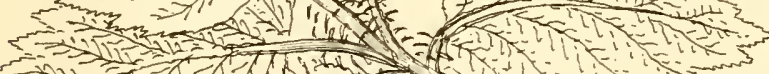

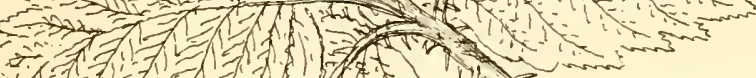

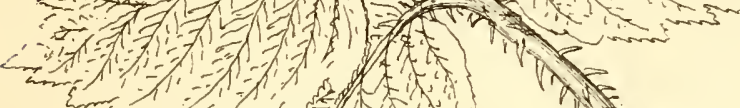

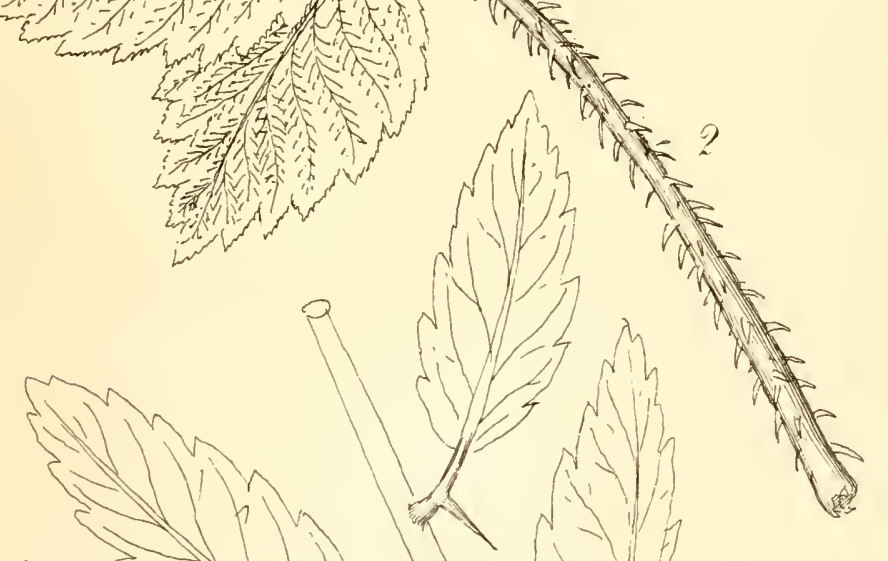





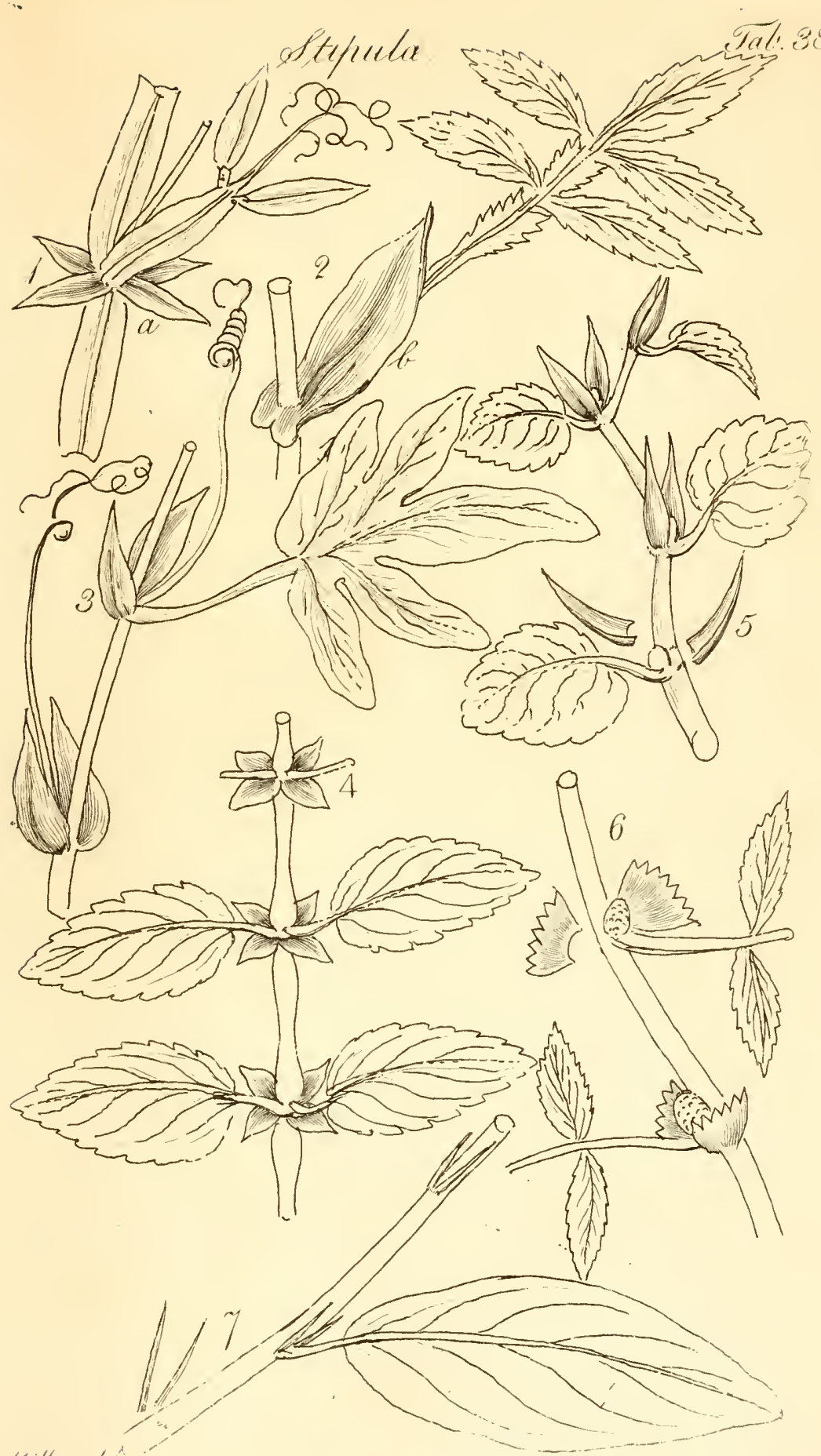





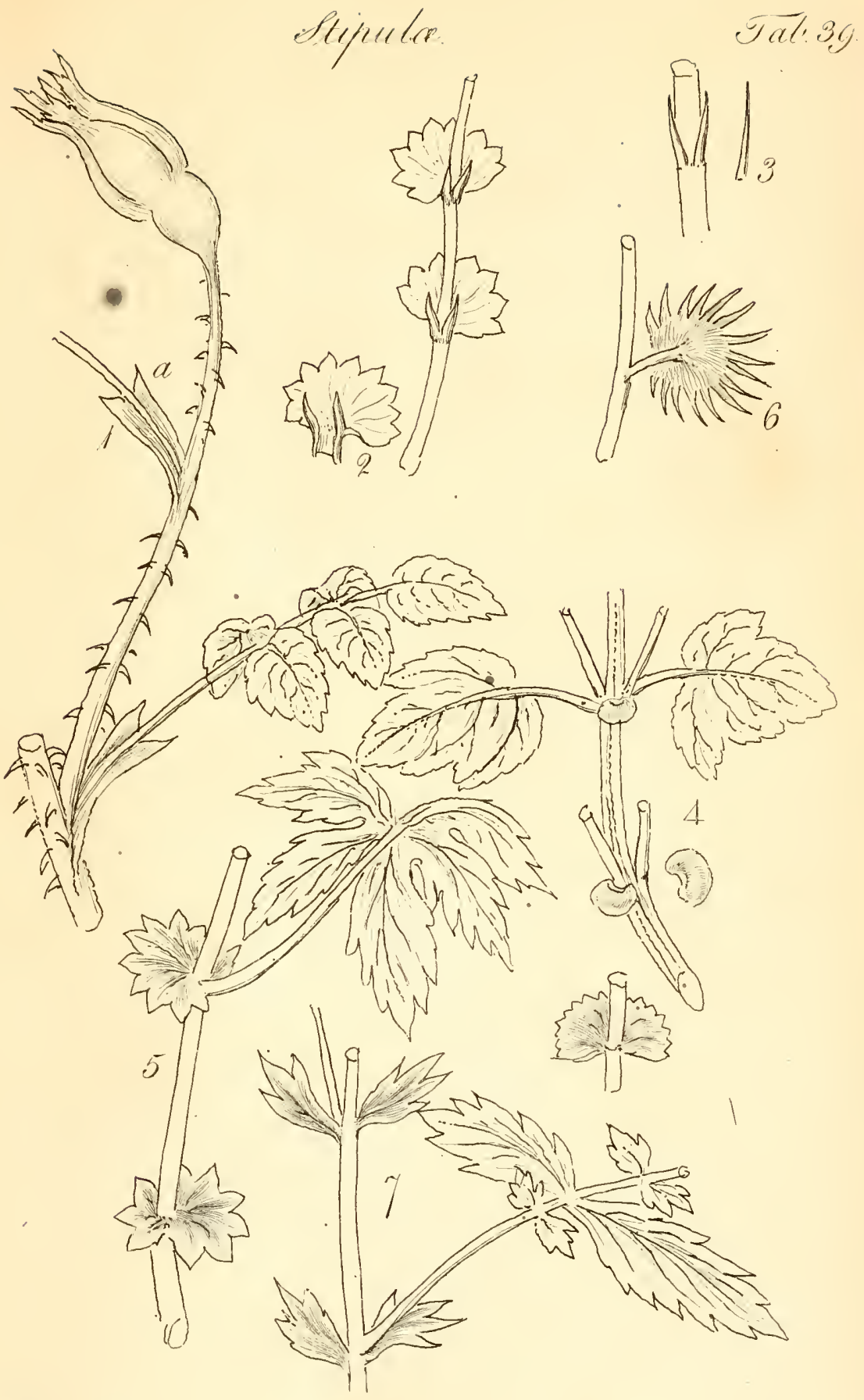

Stiputer

S. Hiller del. 



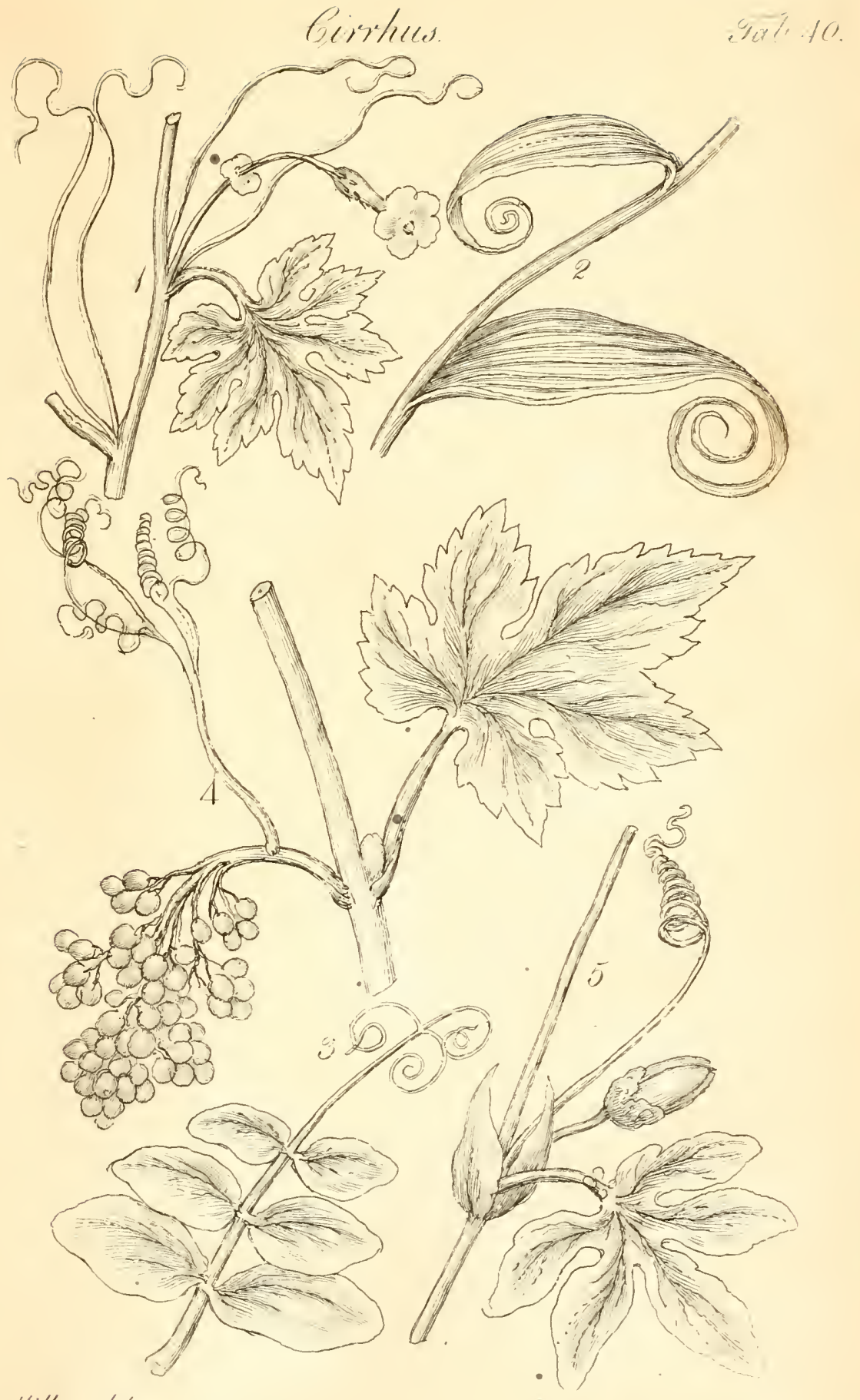

G. Willer del. 



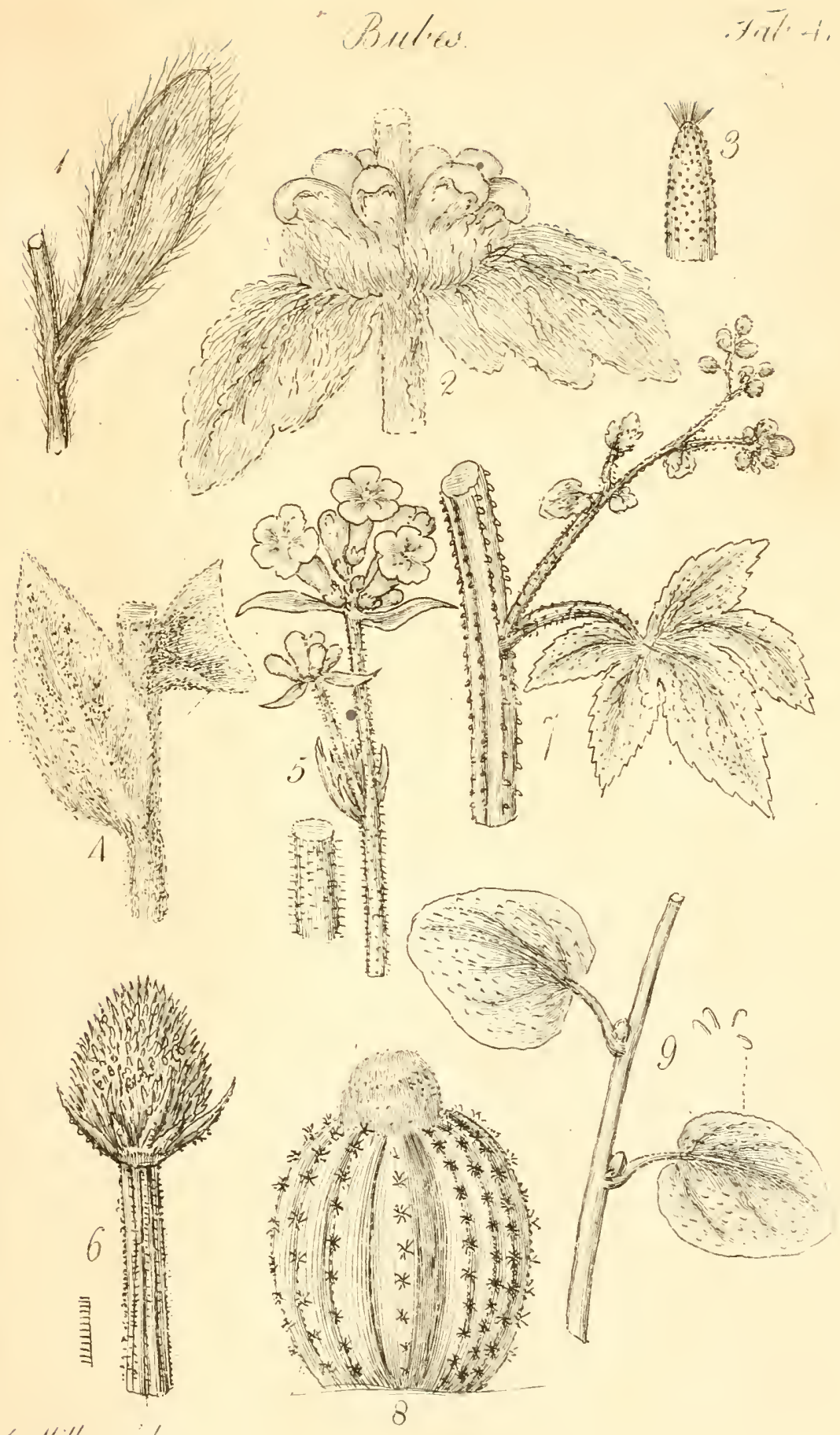





\section{Bubes.}

\section{4 (1)}

Nivi

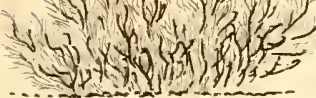

1.
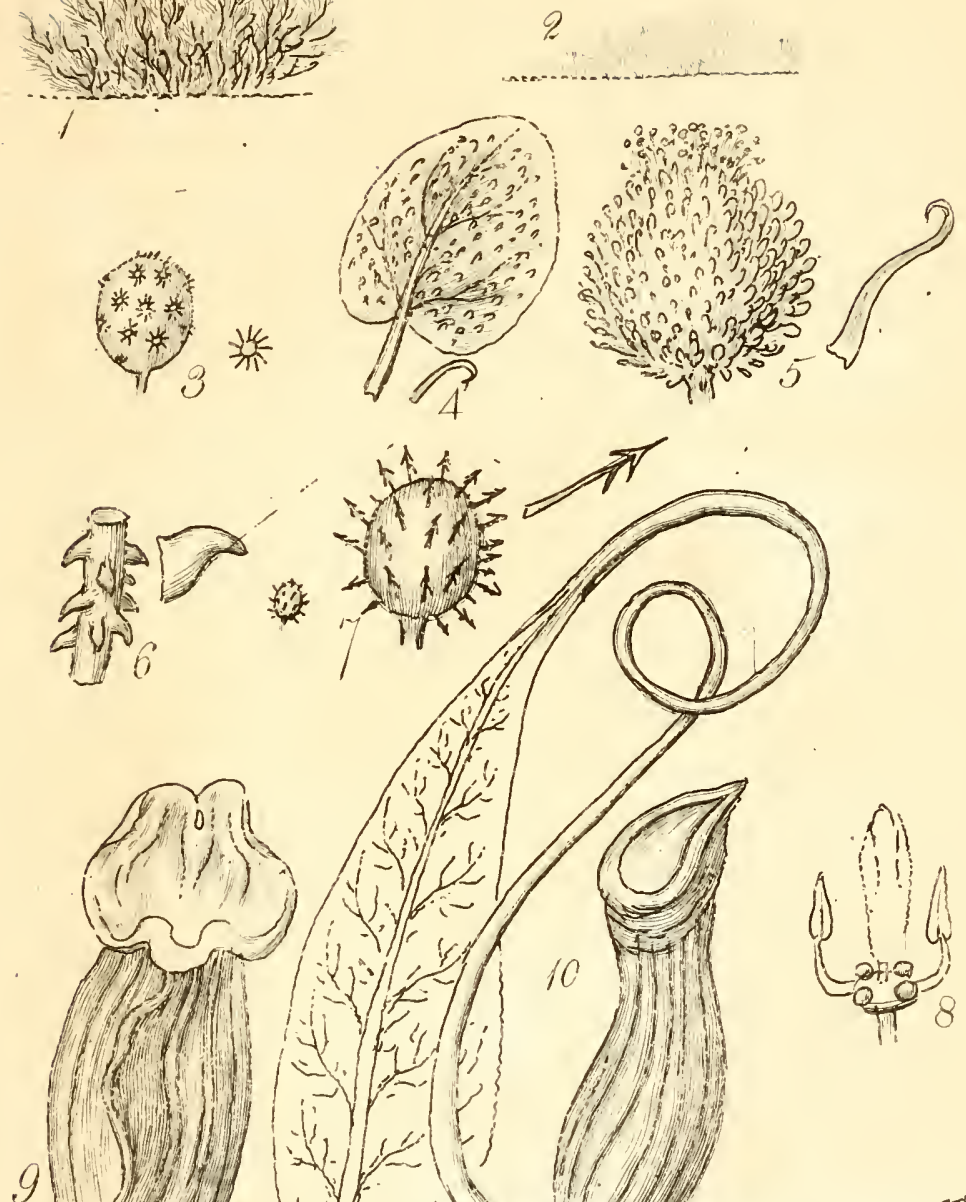

9
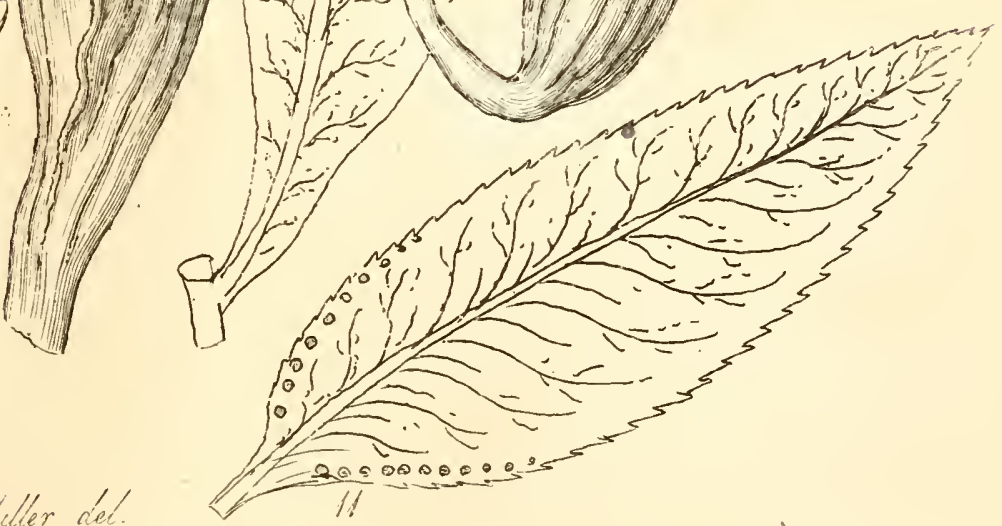

Bulles.

gat. 43

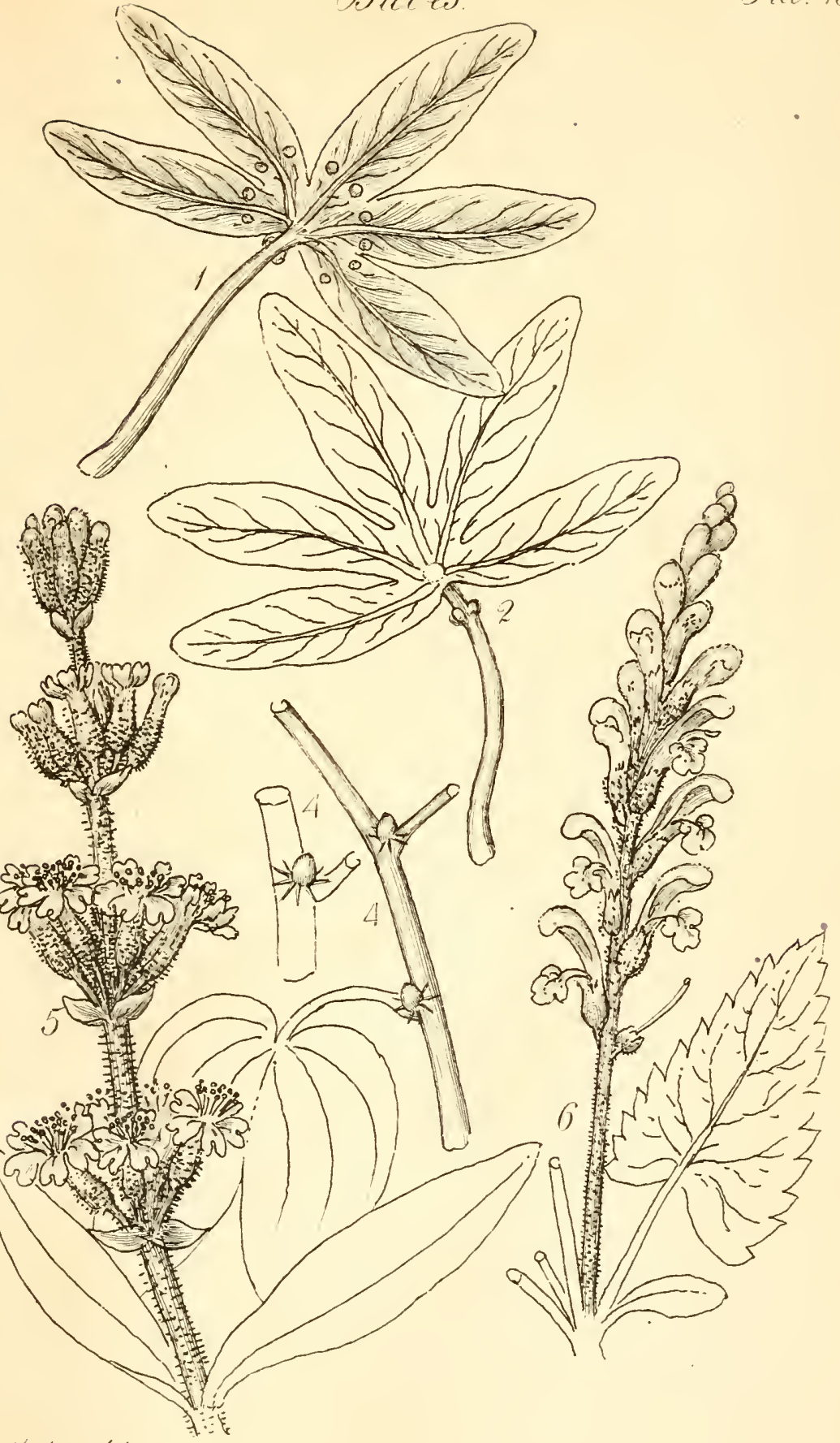

it. illiller del. 



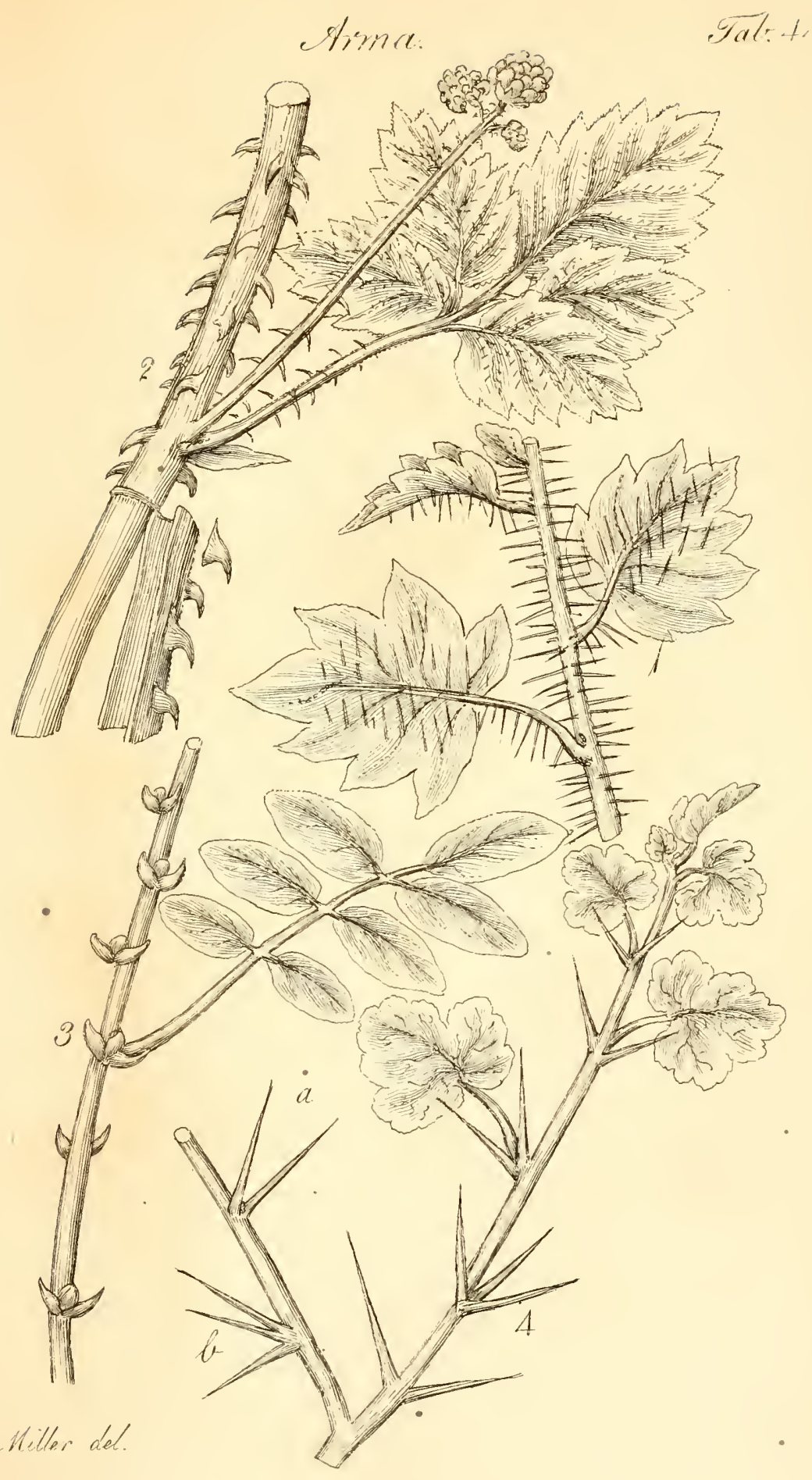





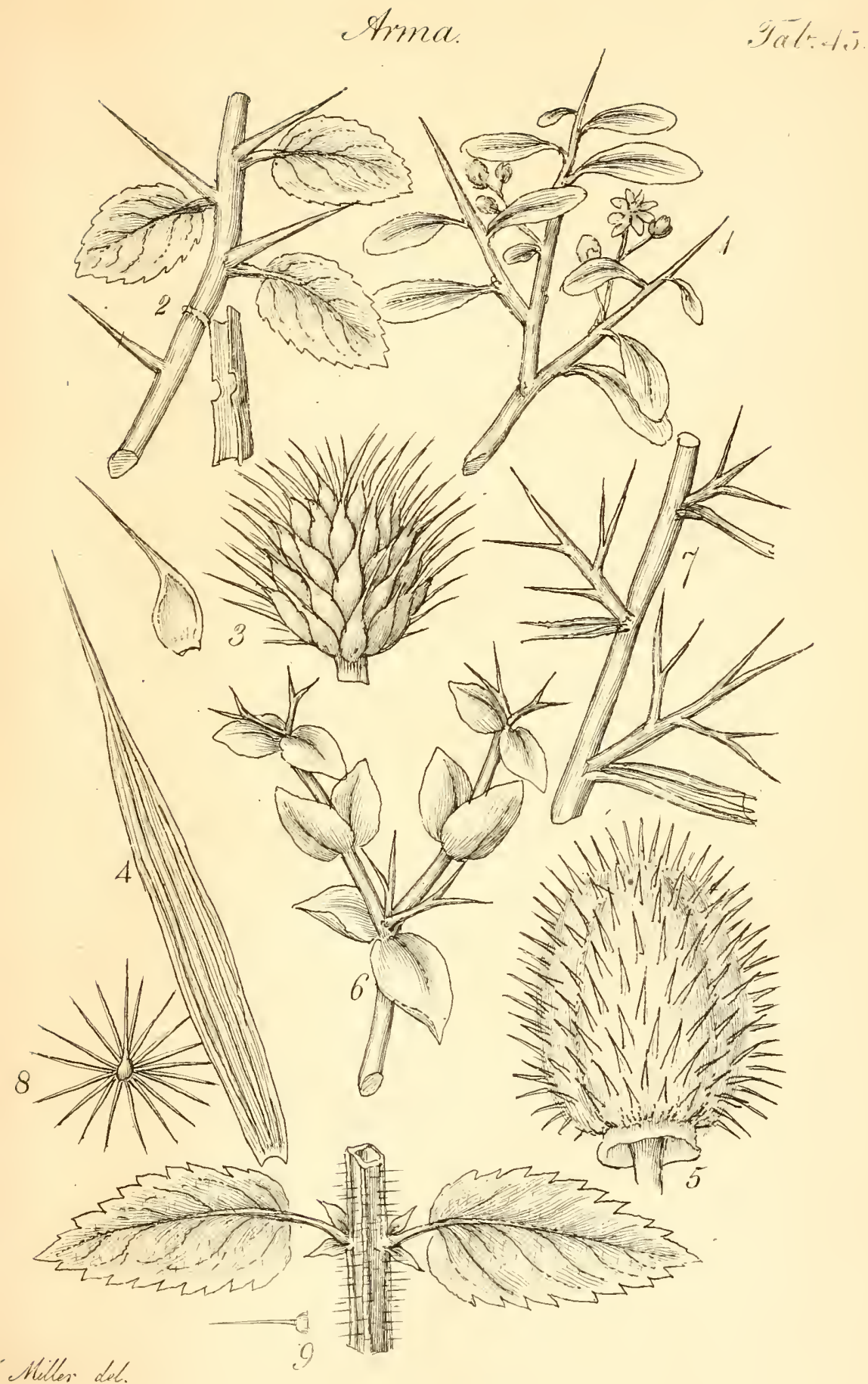





\section{(i) iereter}

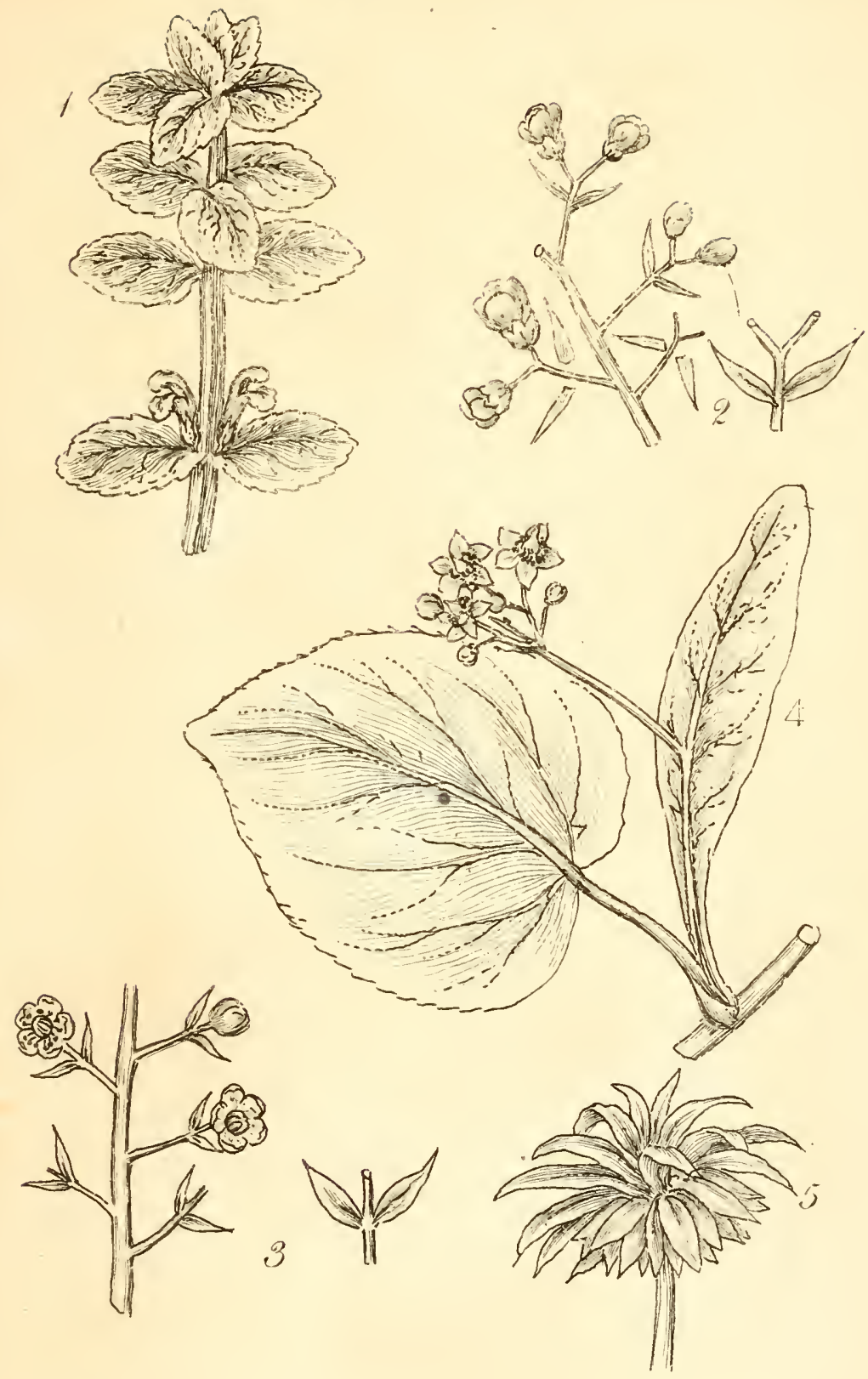





$$
\text { Sónencedes. }
$$
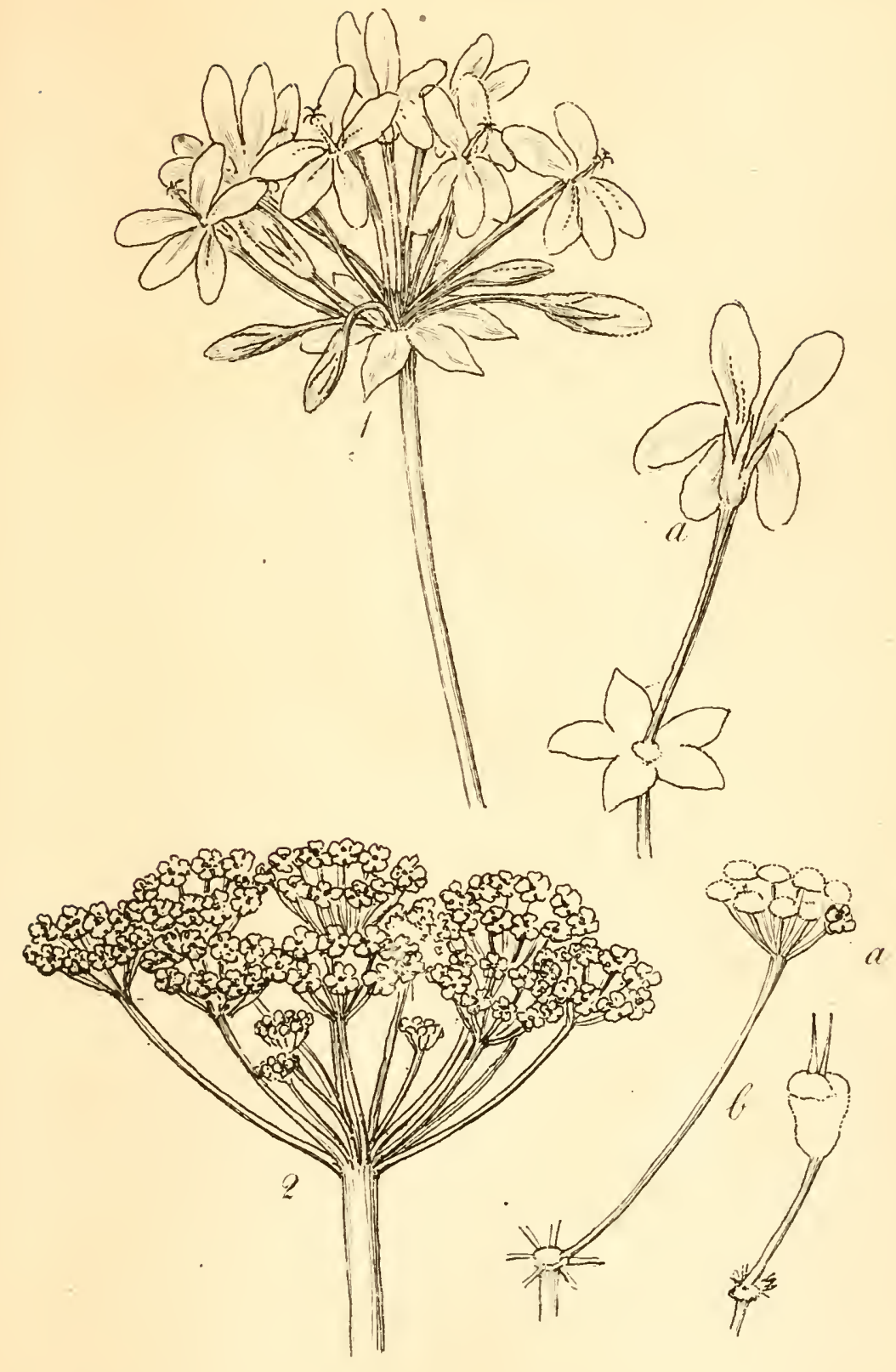



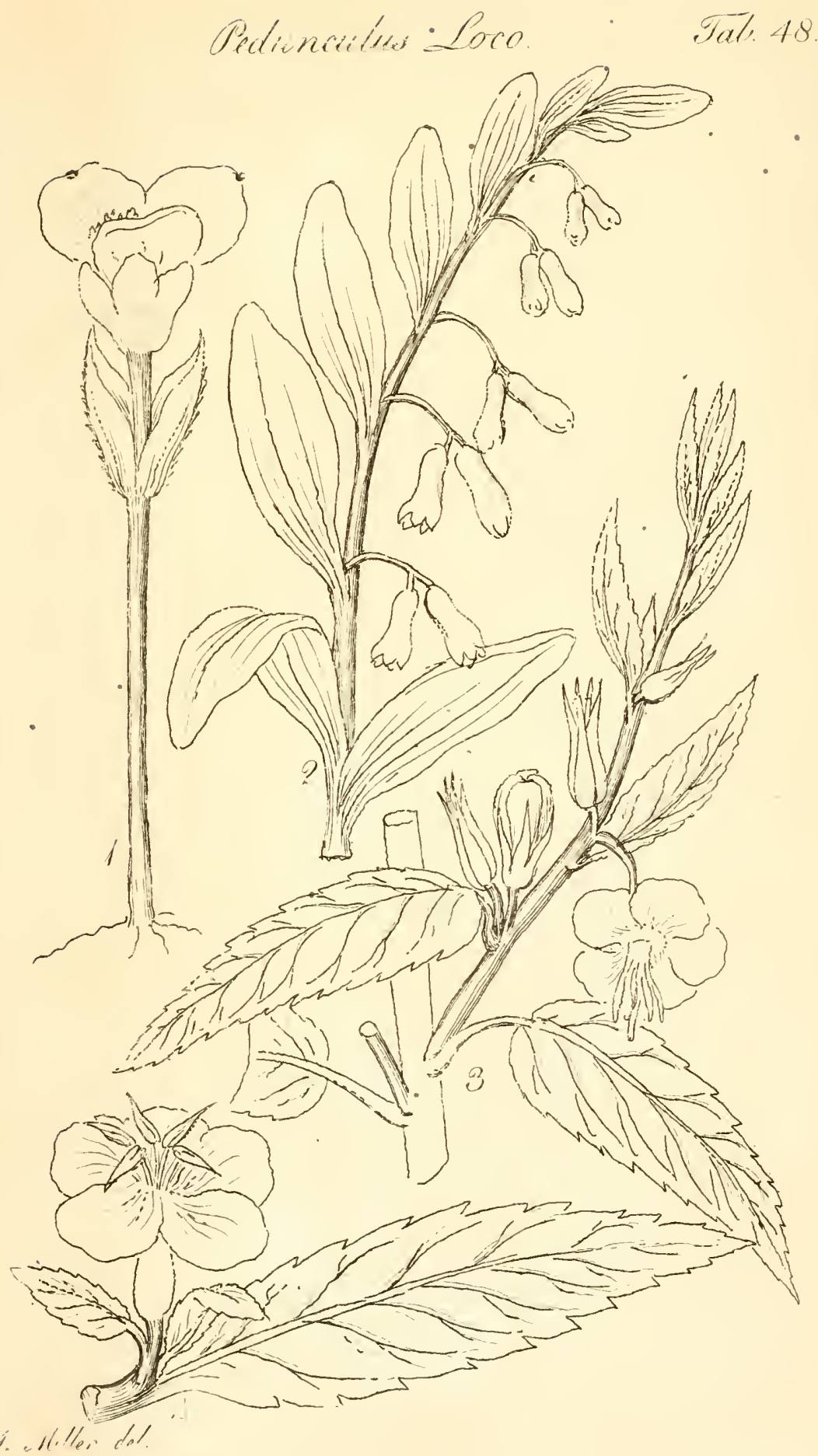



- Pedinculer olóoco
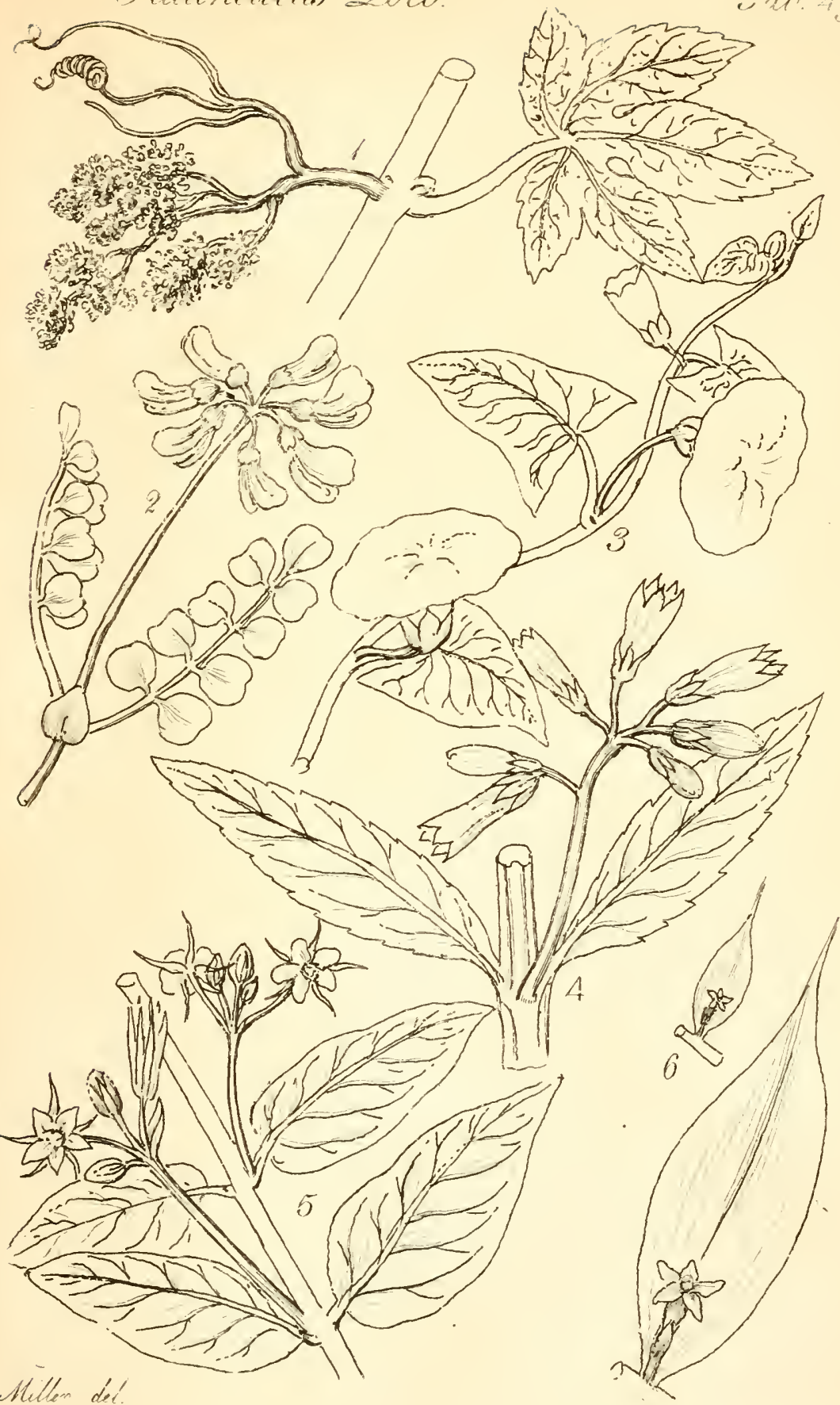

9. Millem dét

(i) $20=$ 



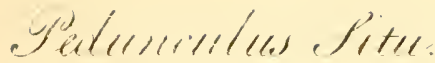

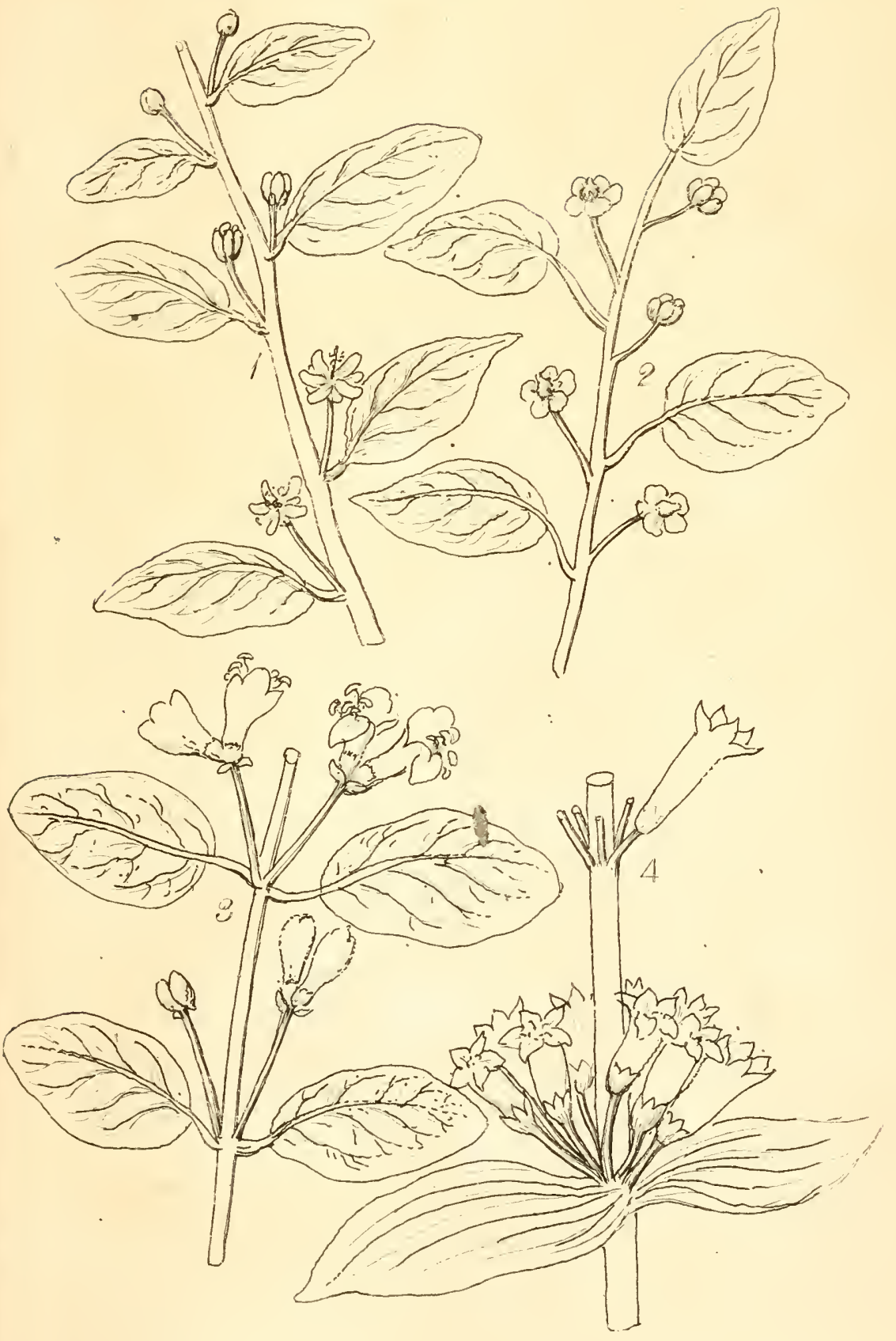

c. Hiller del. 



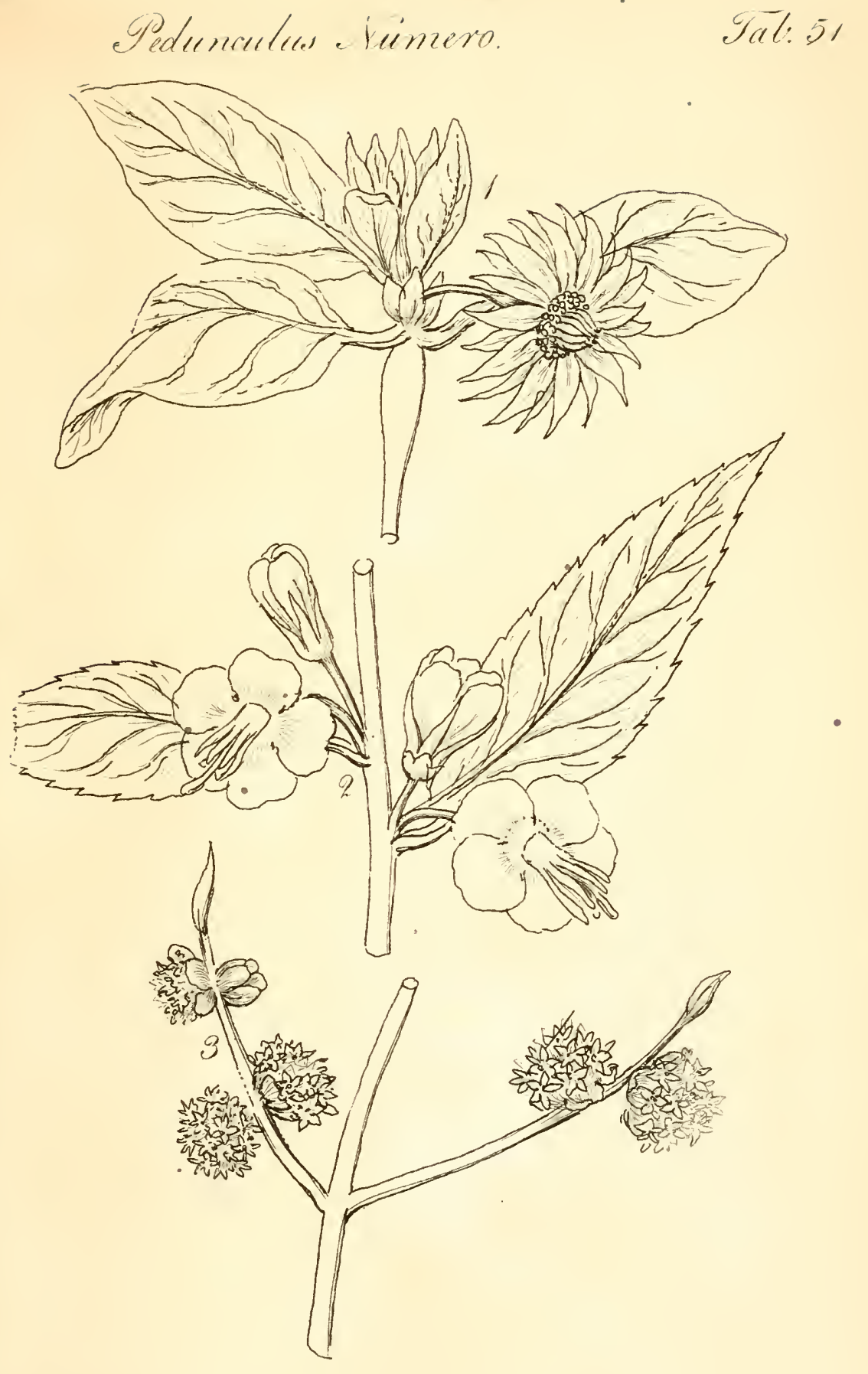

S. Miller del. 

Södunceler, Zirection.

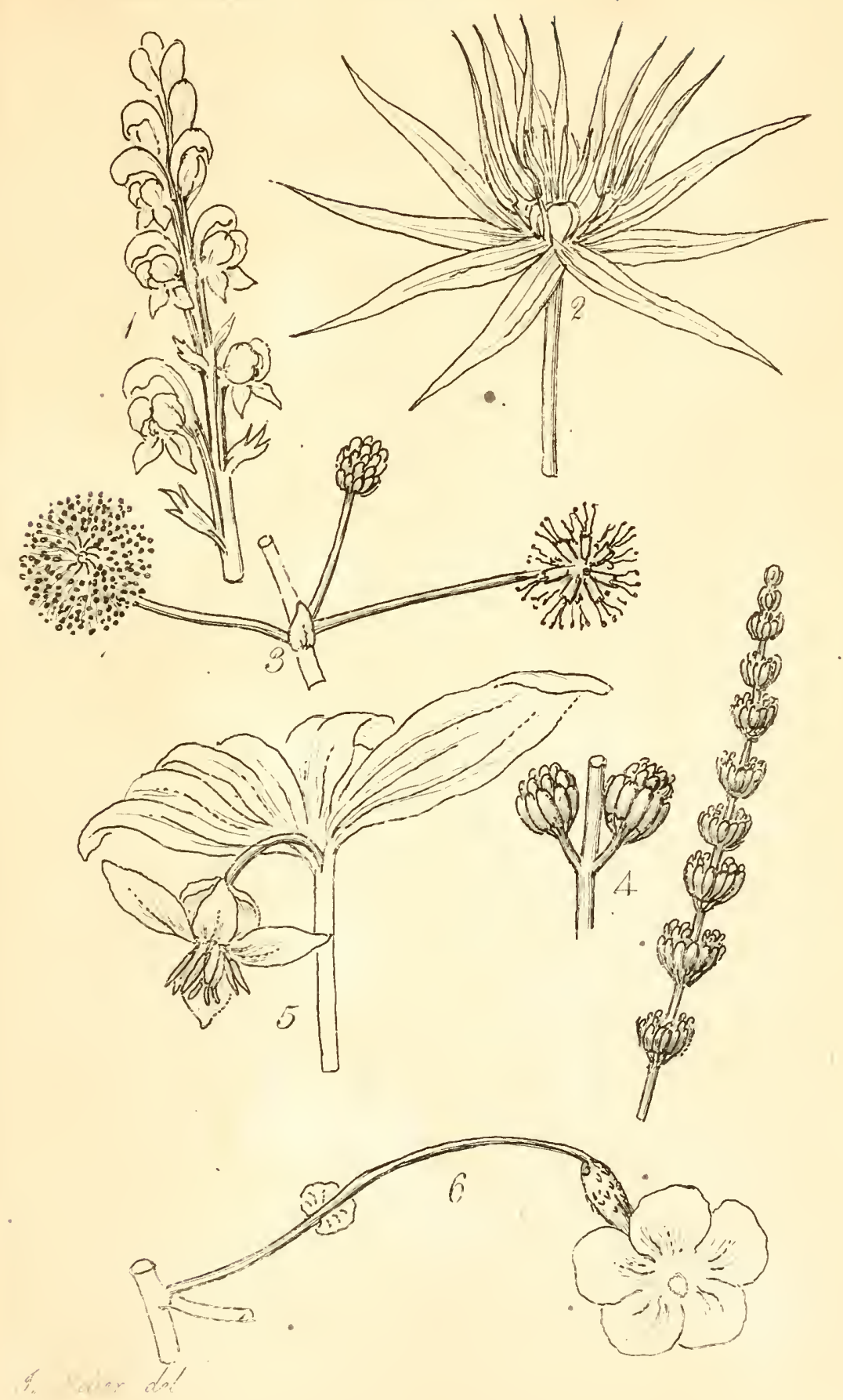





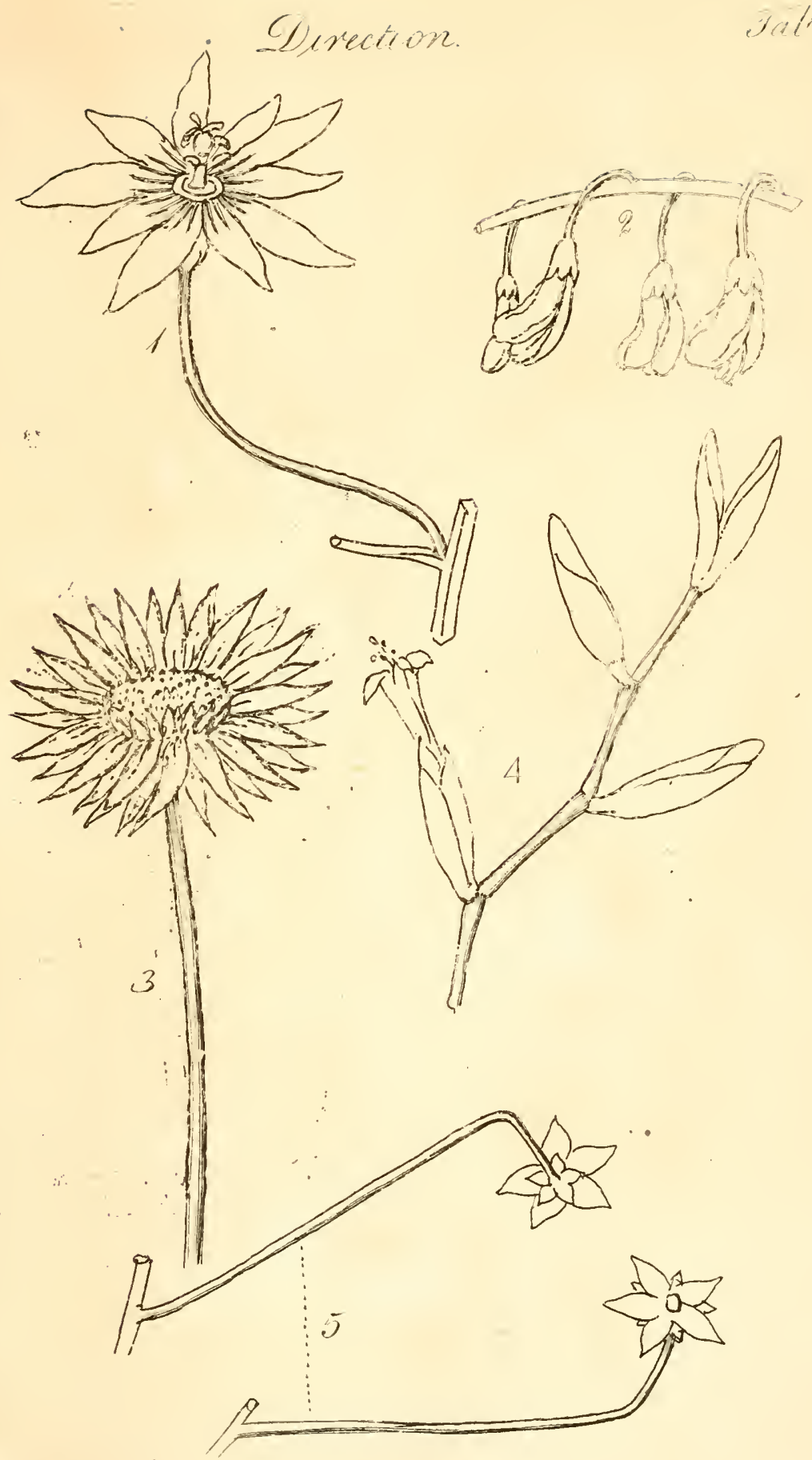

I. - lleller del. 

Sedenculus Siñuctura.

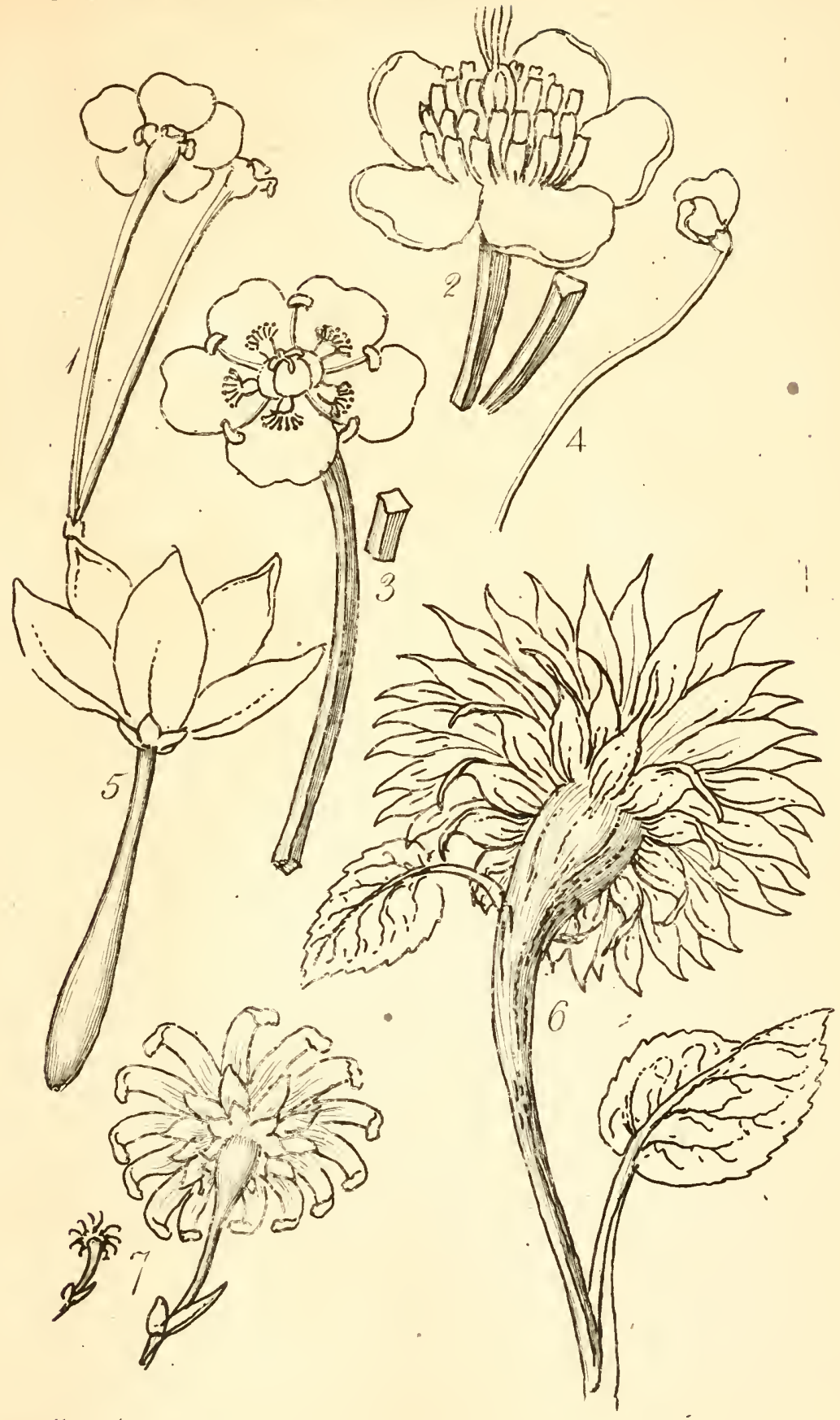





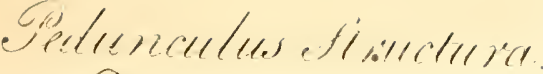

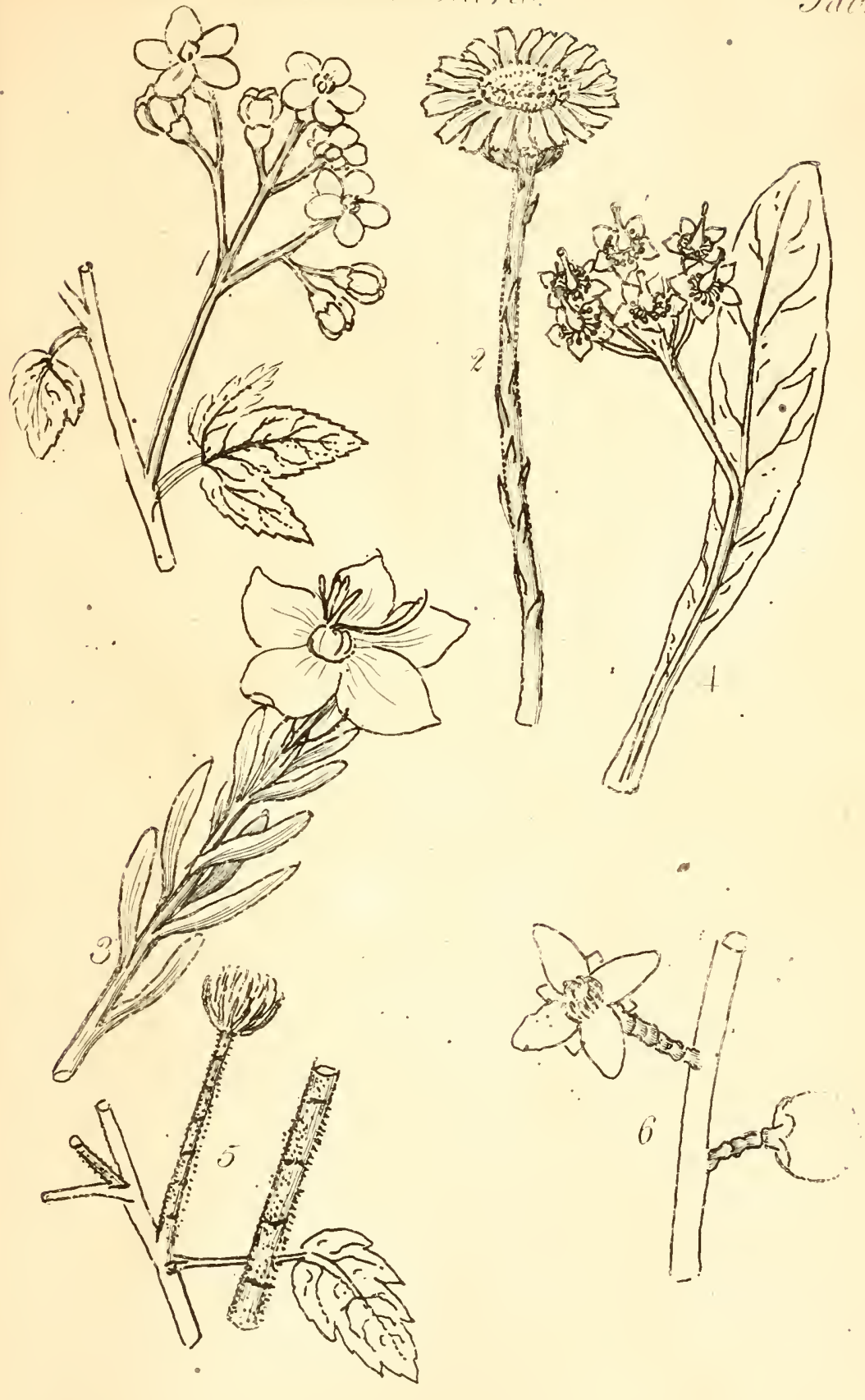





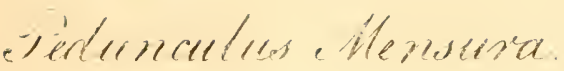

TतT. 56
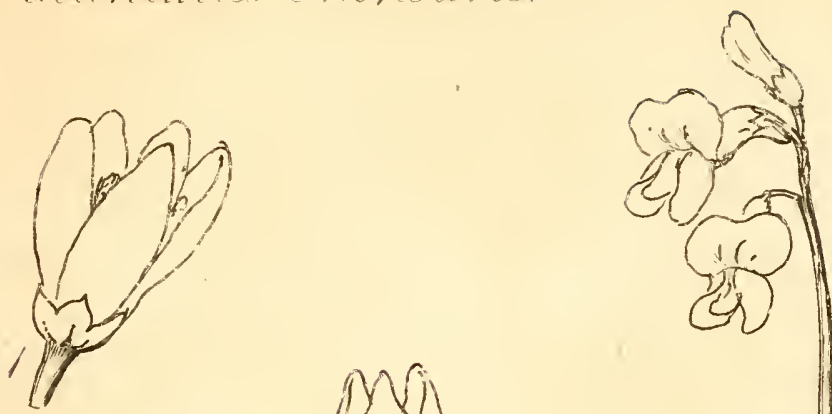

Sf
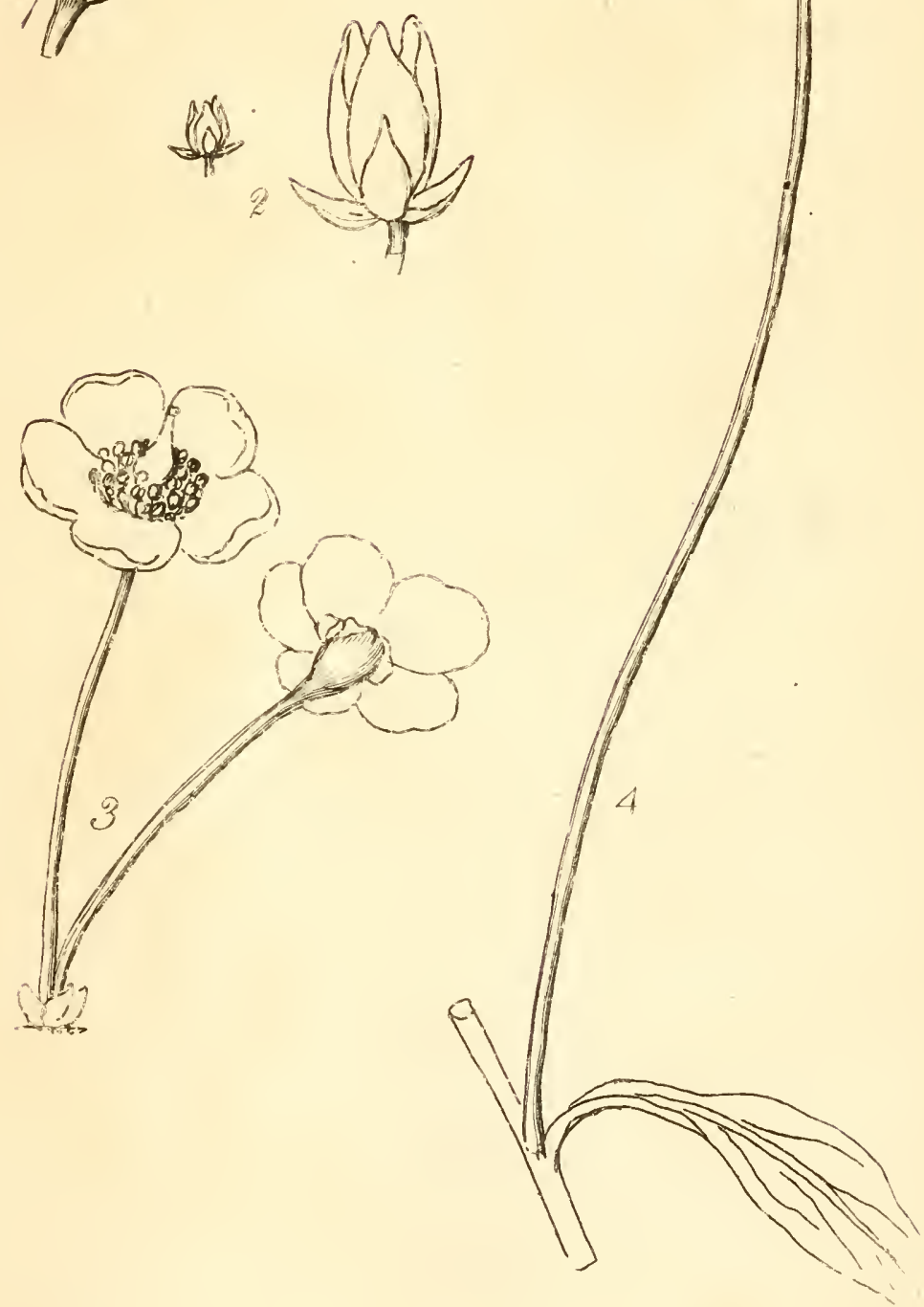

Inflorescens.

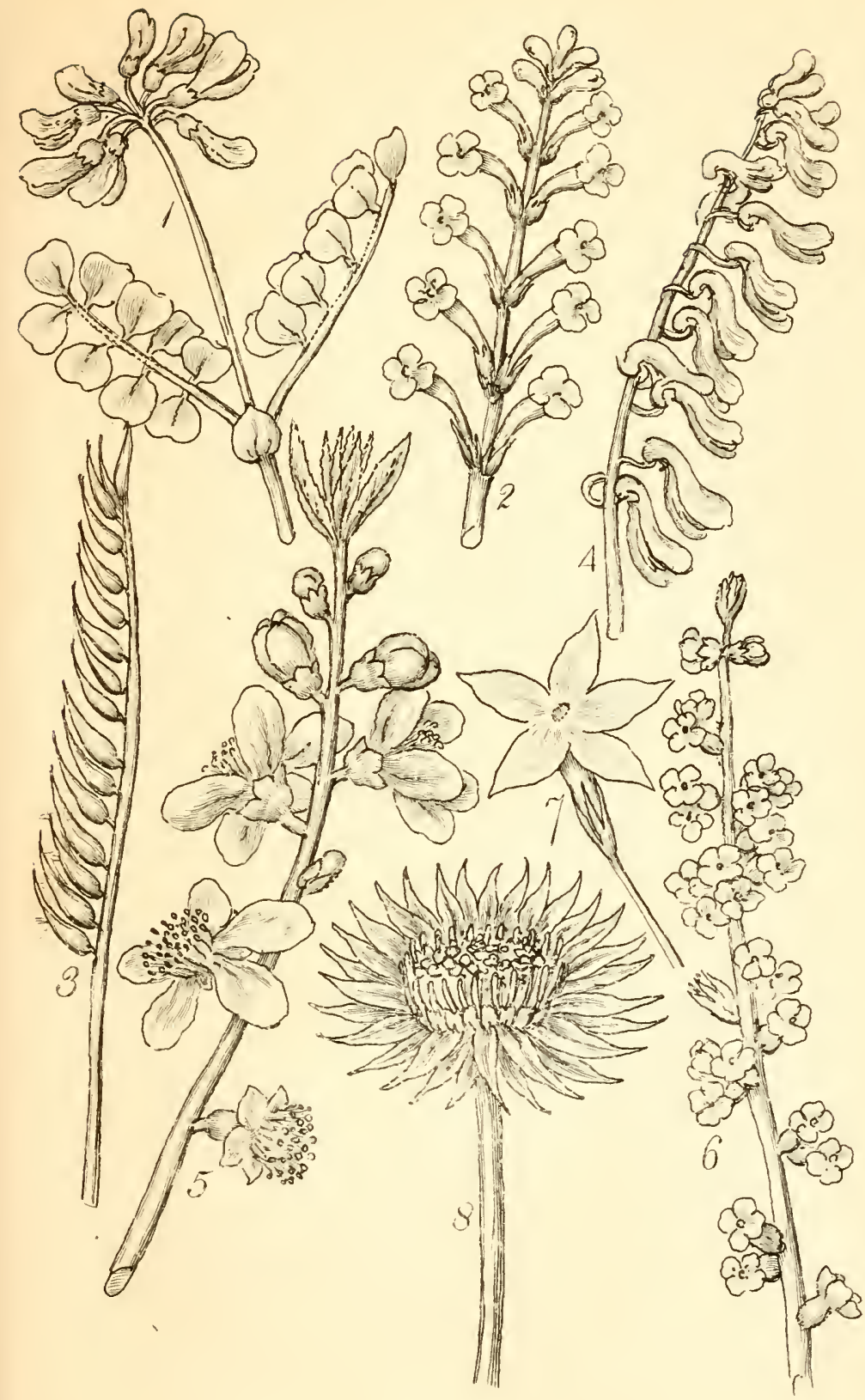





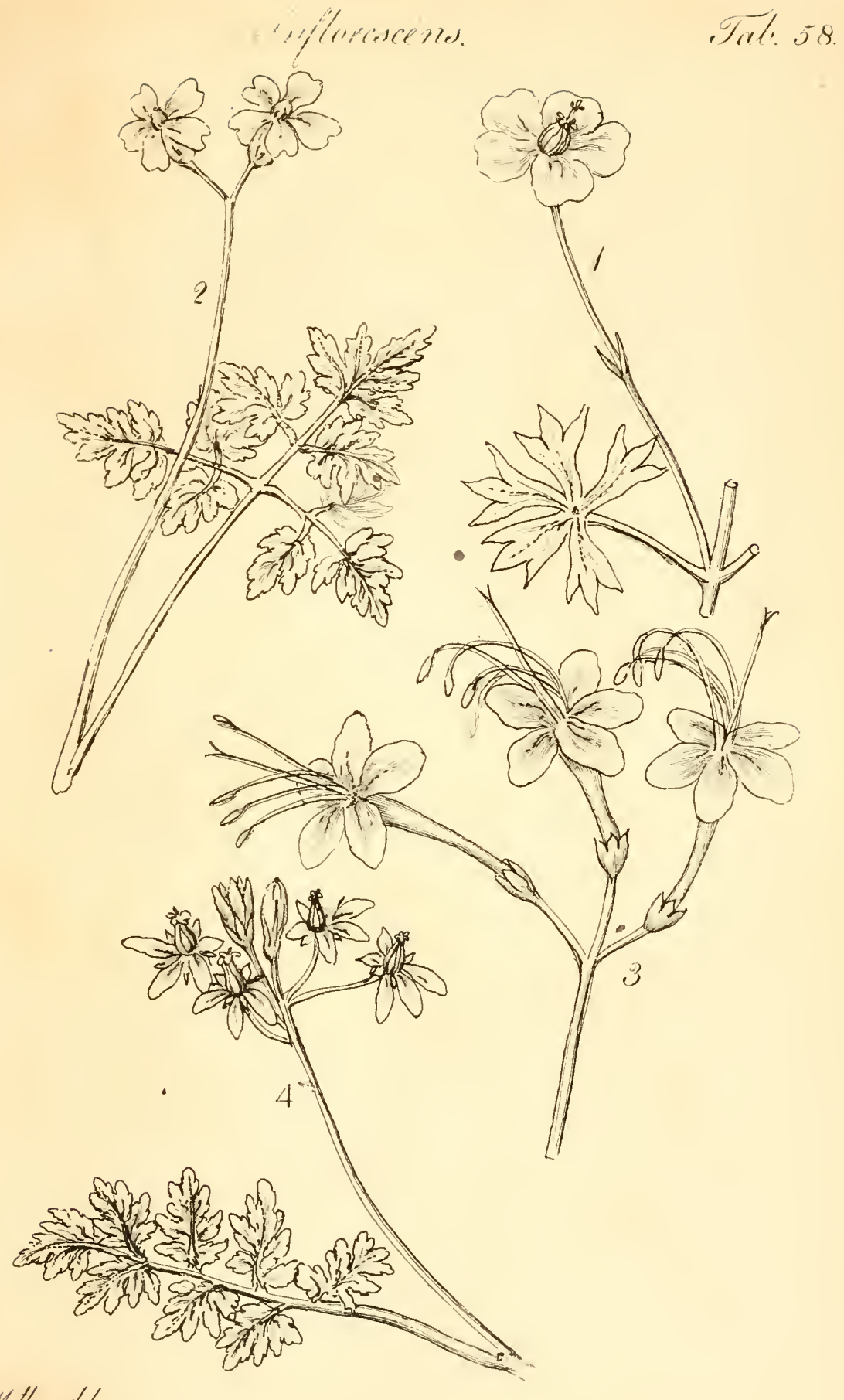

A.cuther del. 



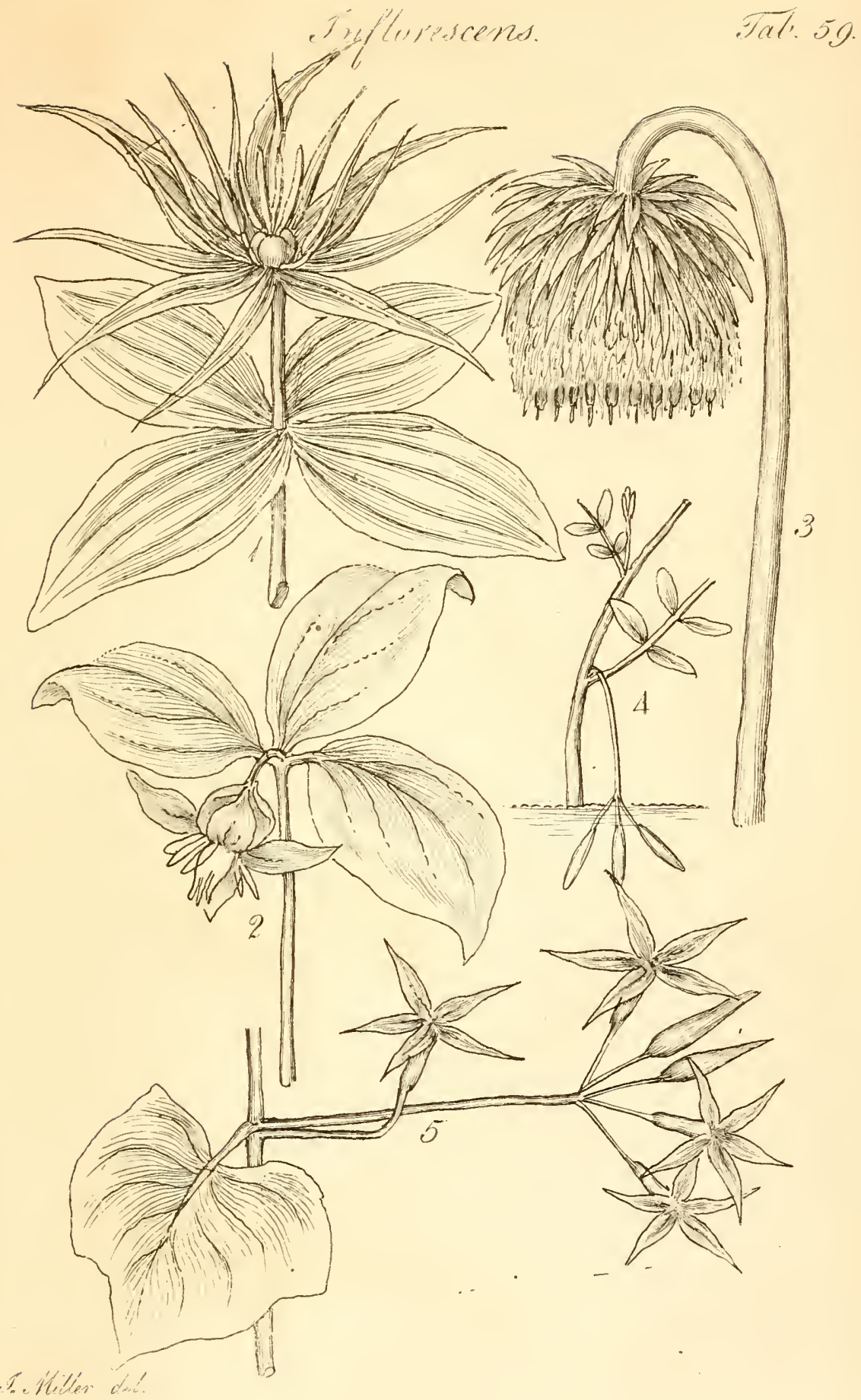





\section{levticilles}

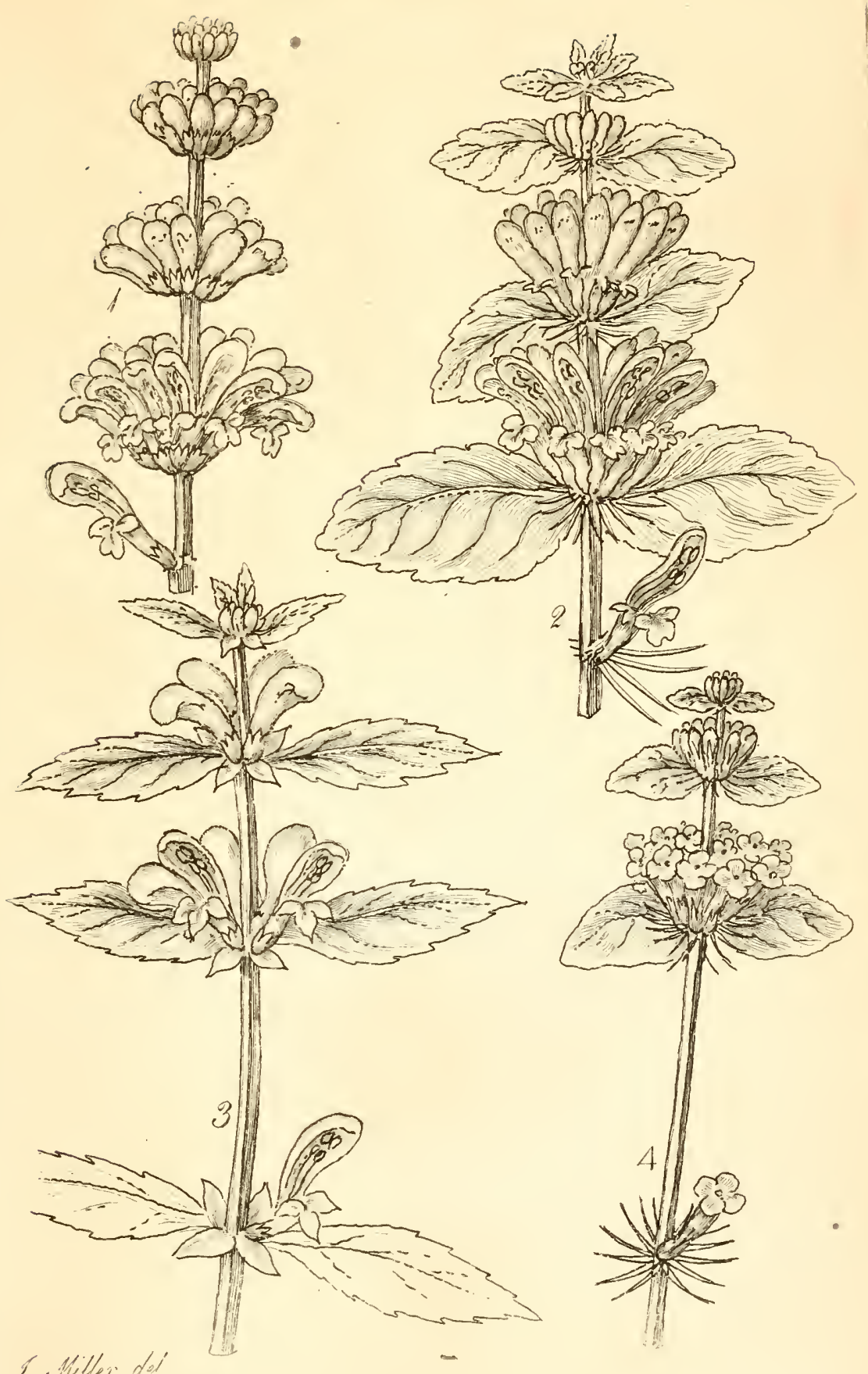




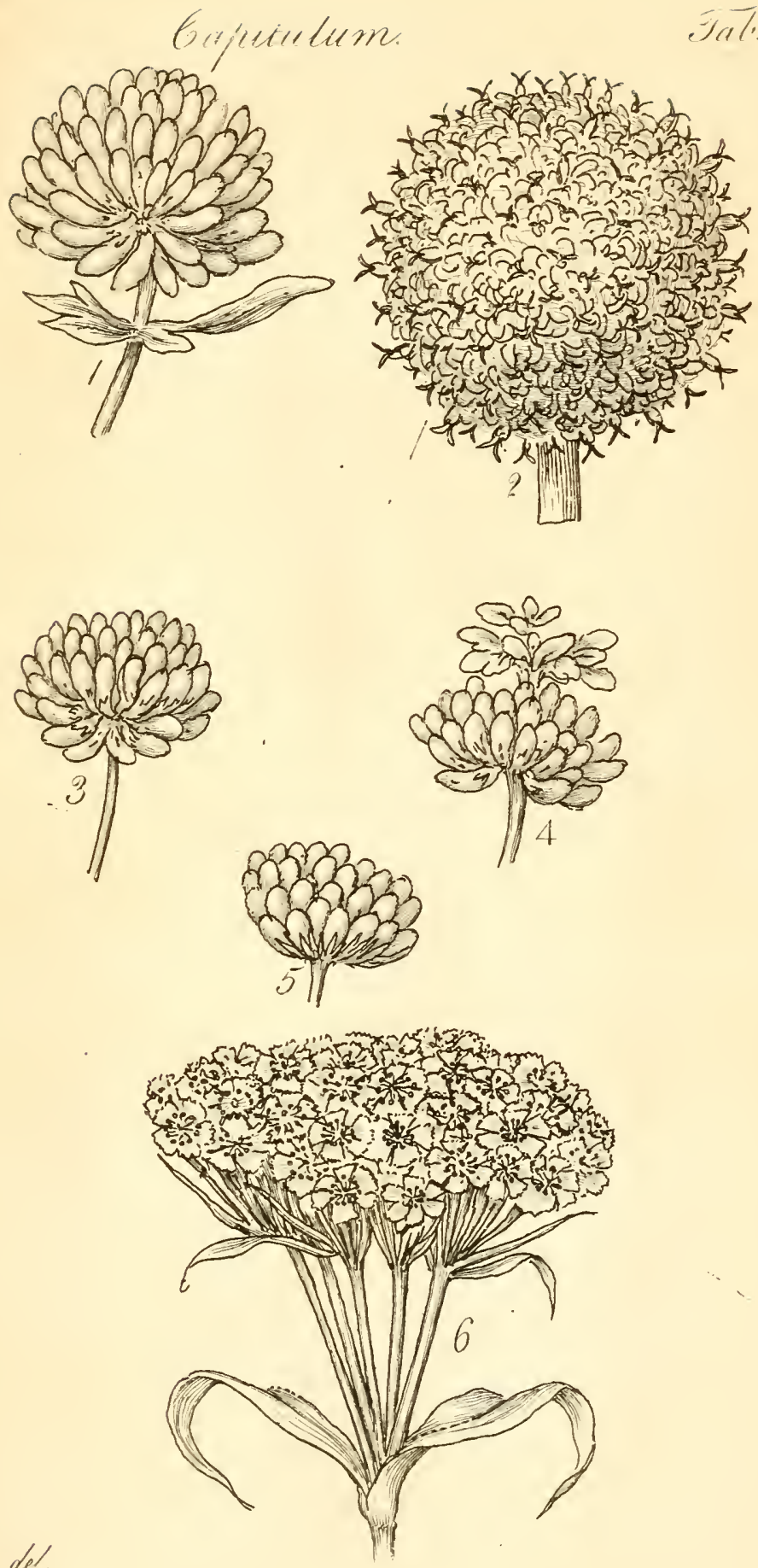



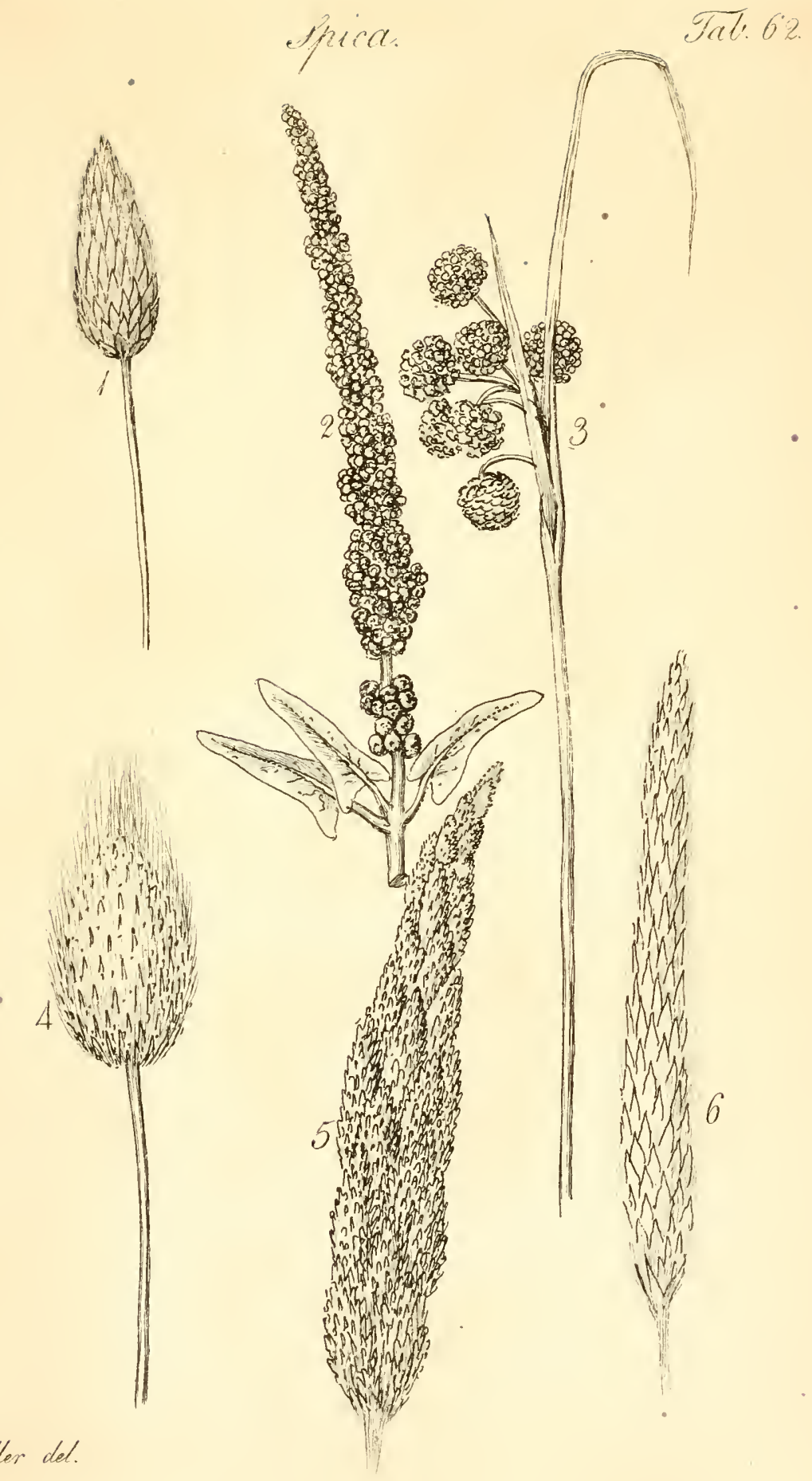



suce.

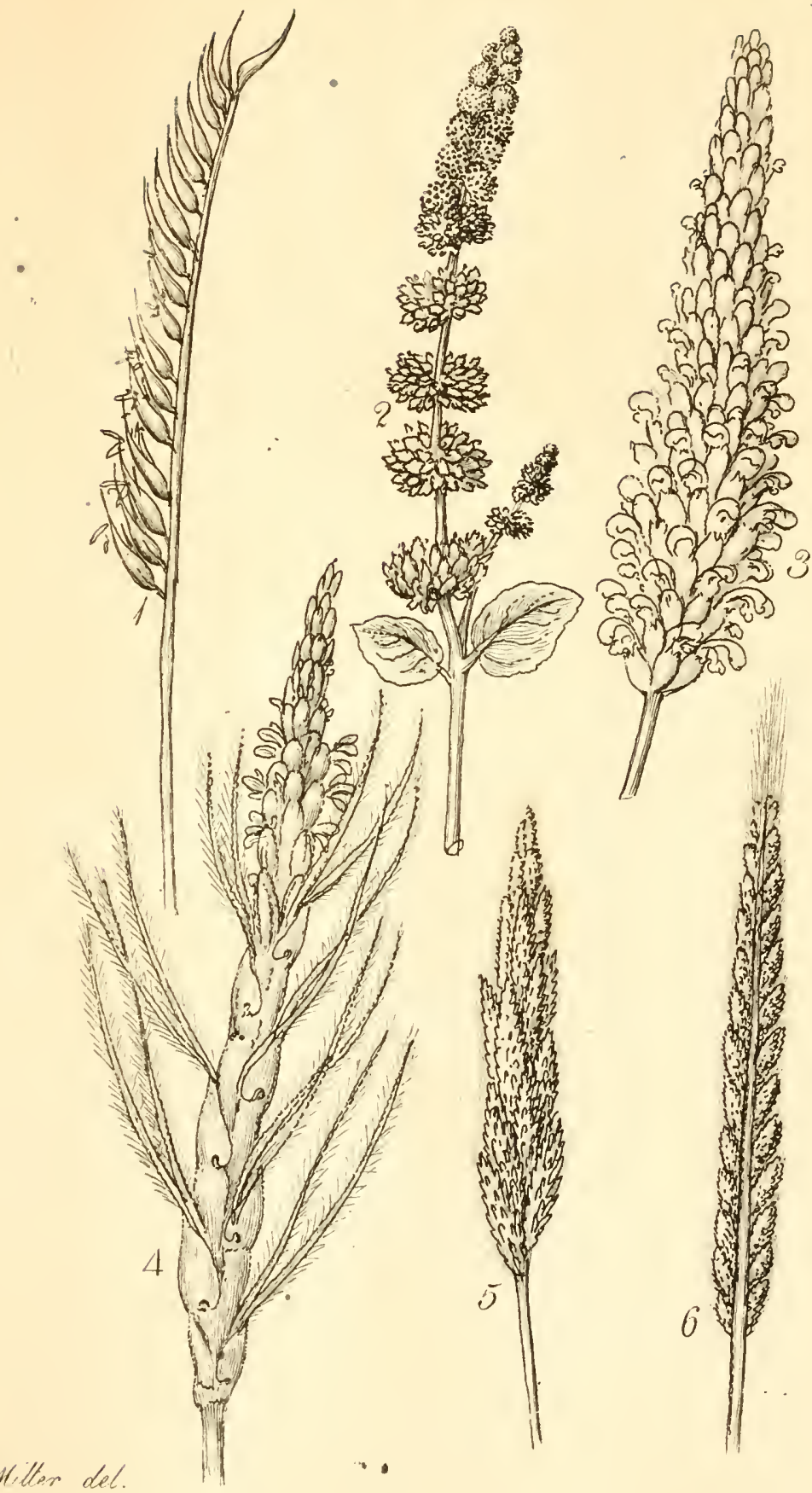



trucd
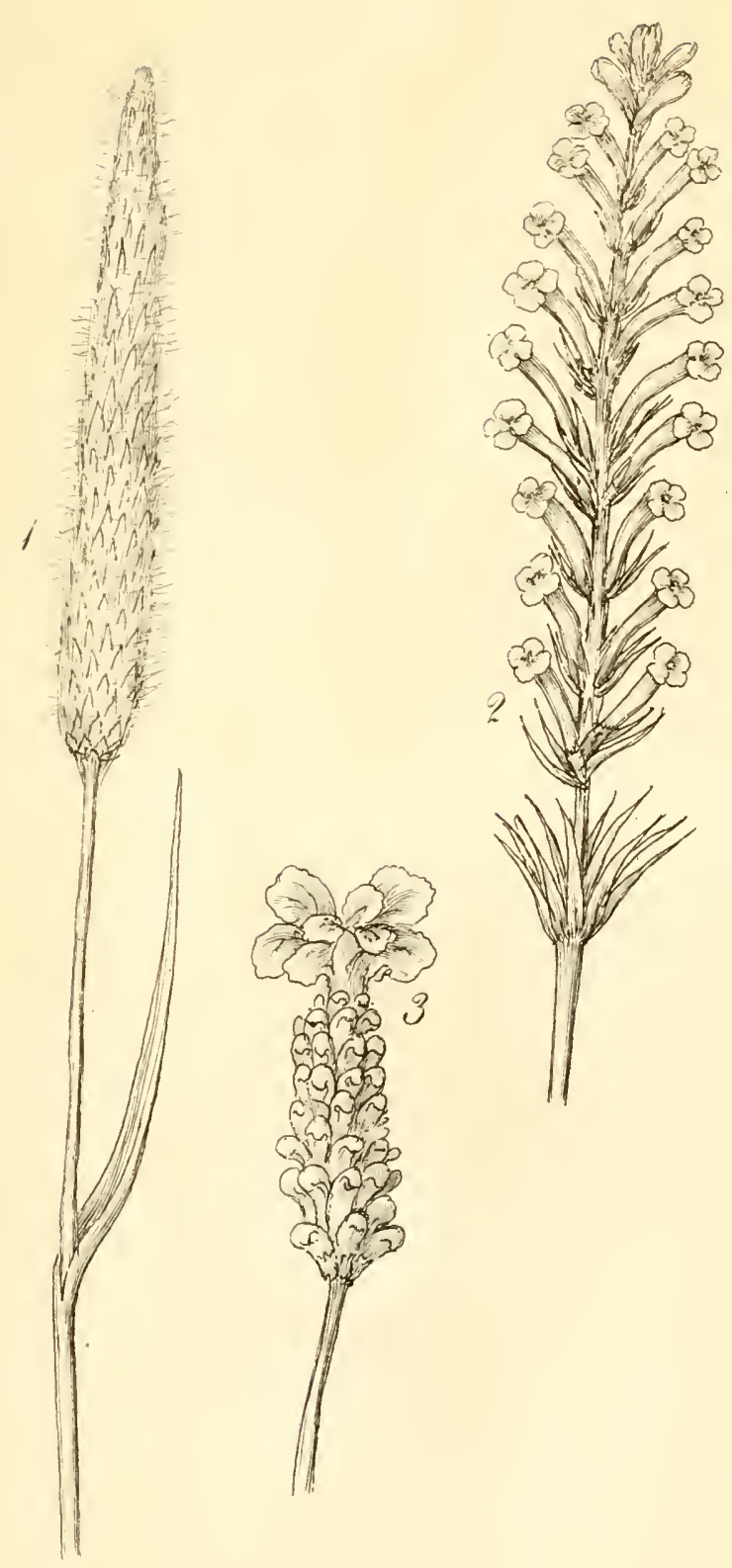


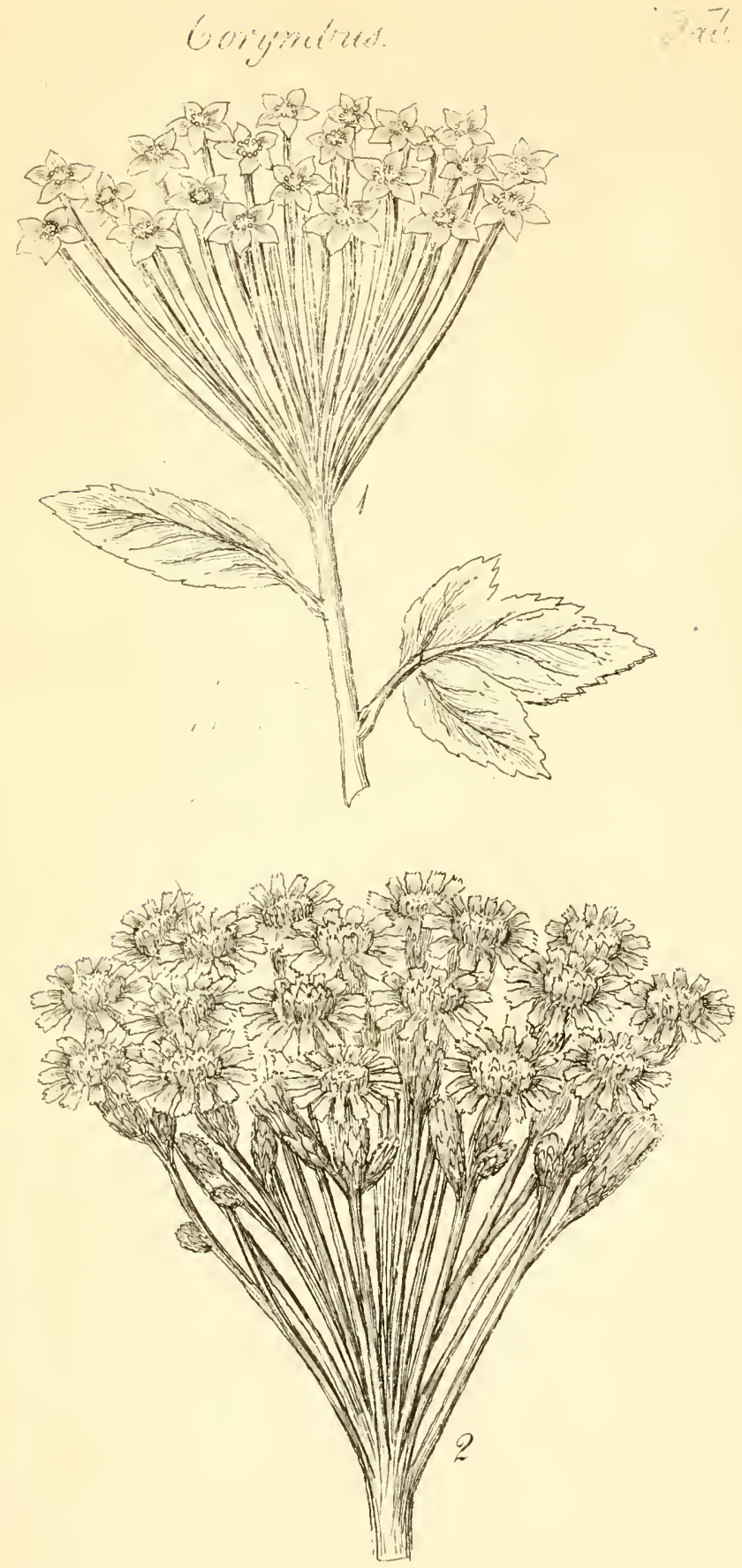

S. Willer del. 

Thiprest.

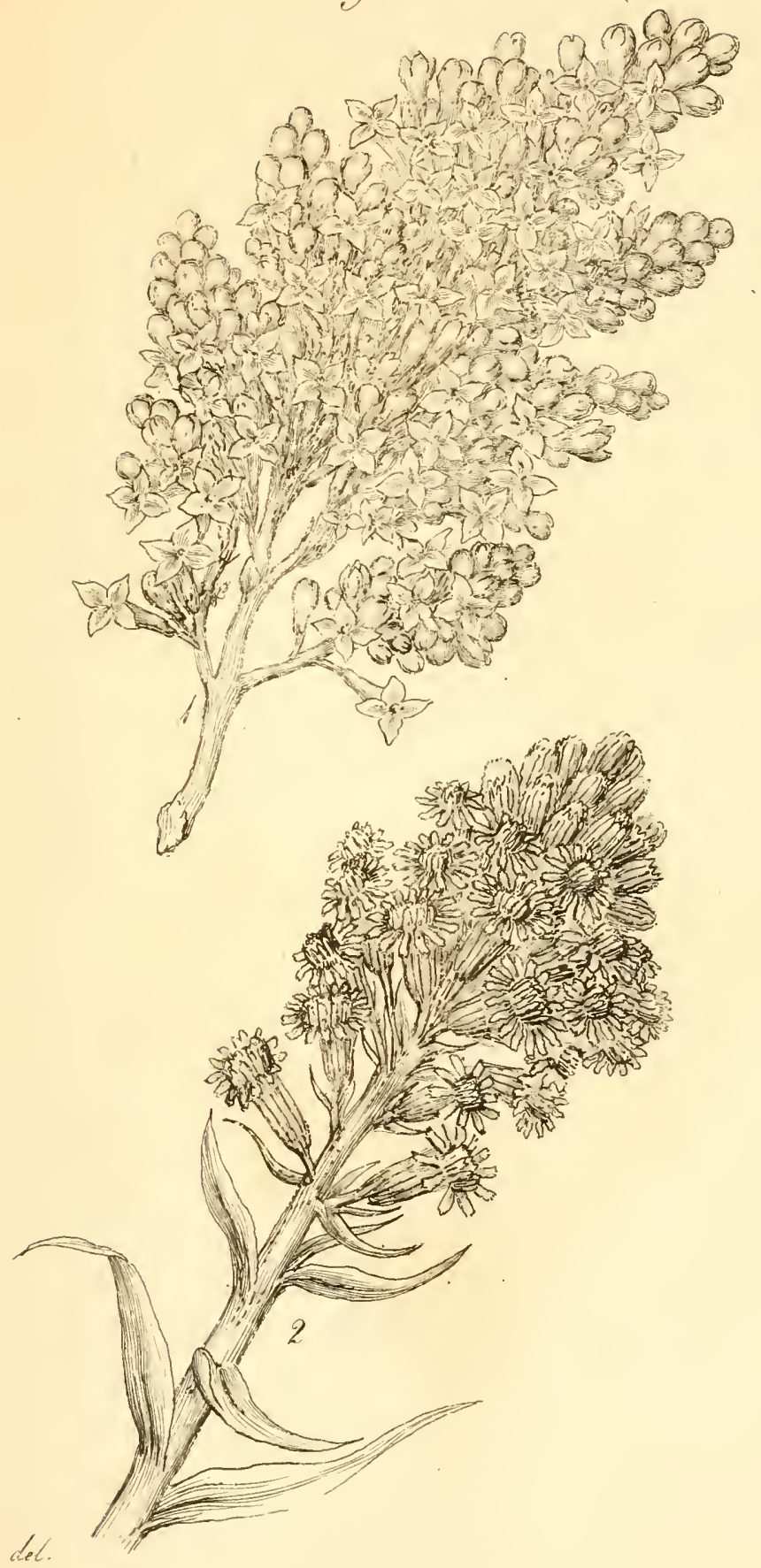

Yá 66

- 

Racemetes.

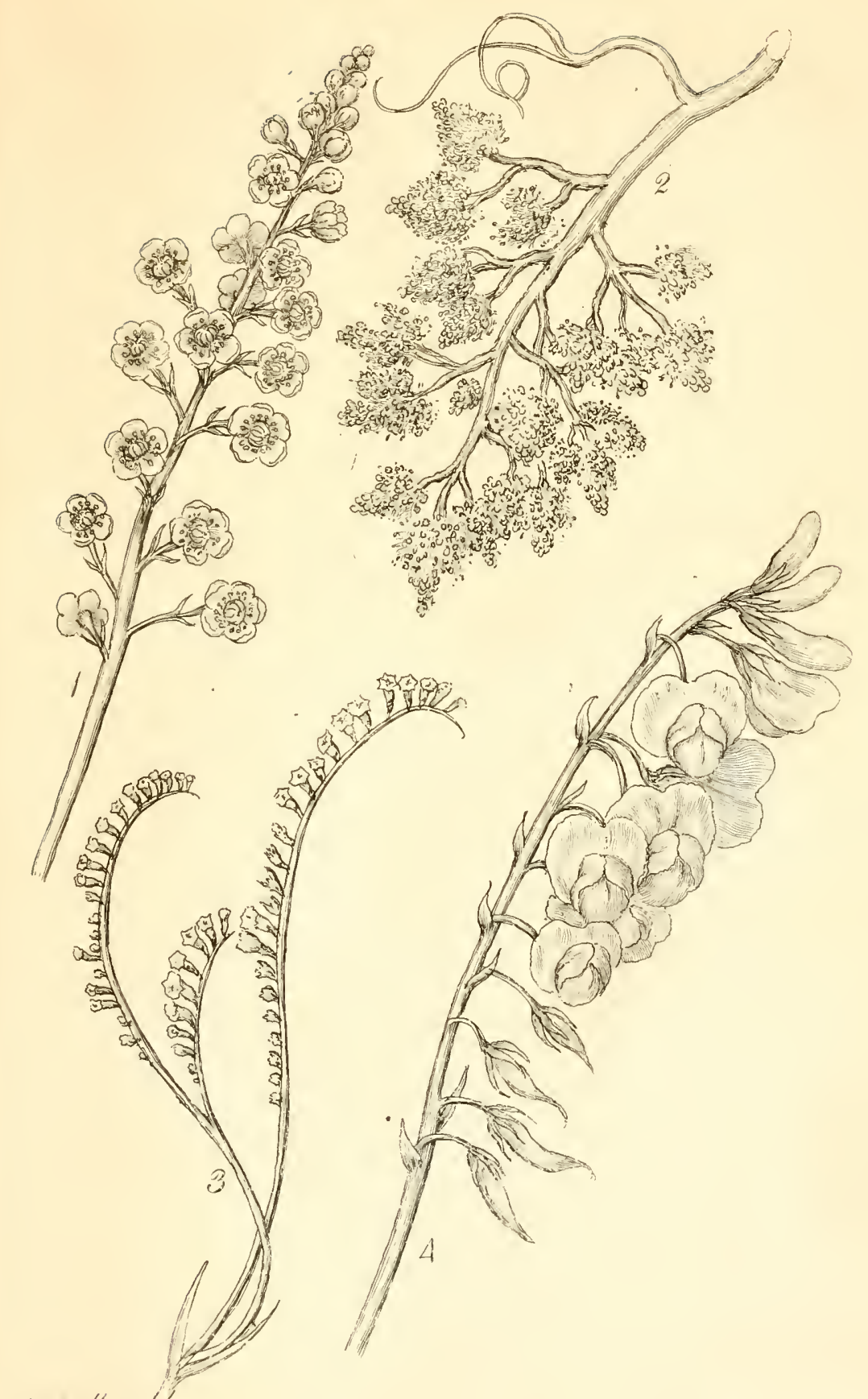





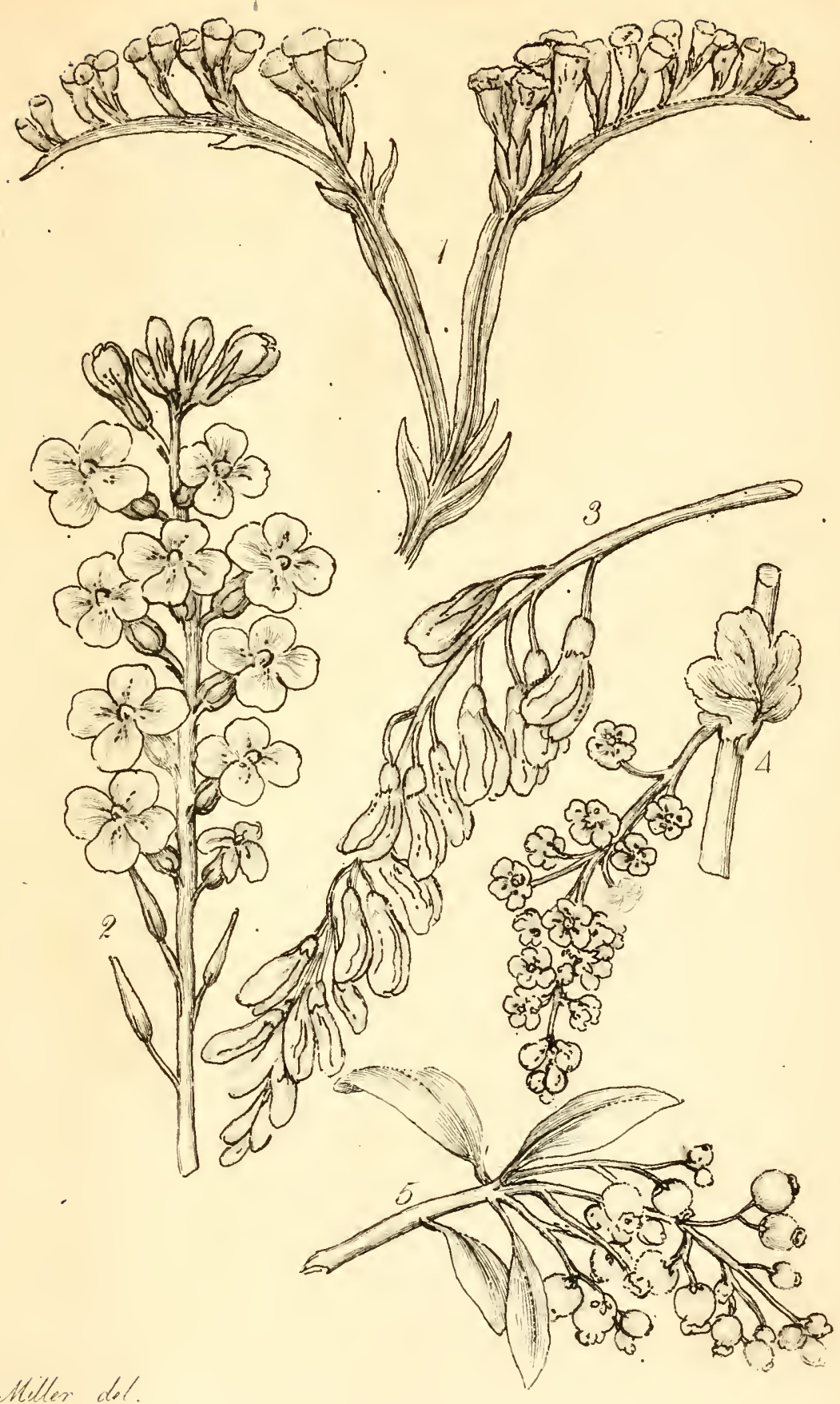





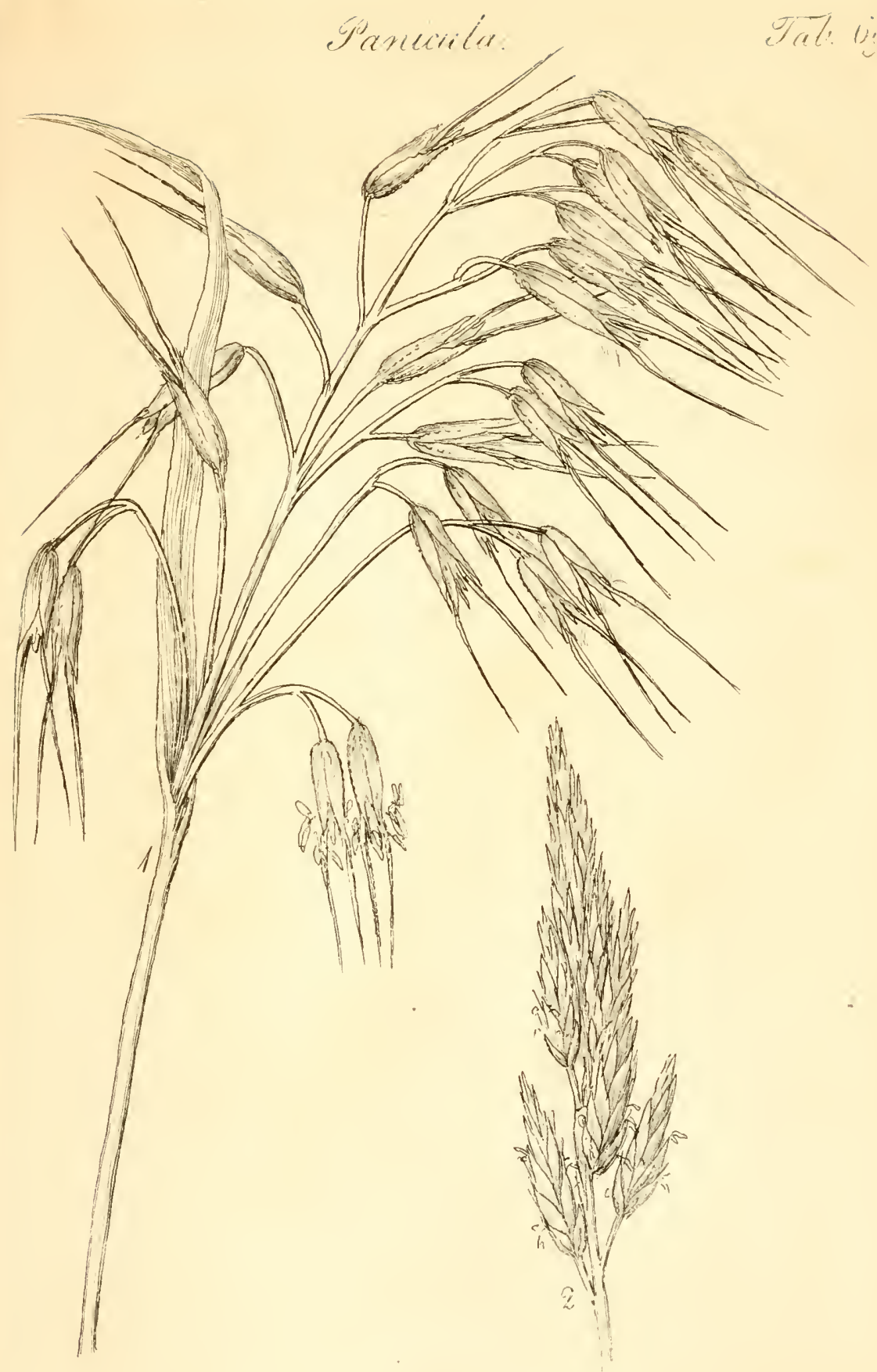

1. Heller del. 



$$
\text { Galyx. Saligo }
$$
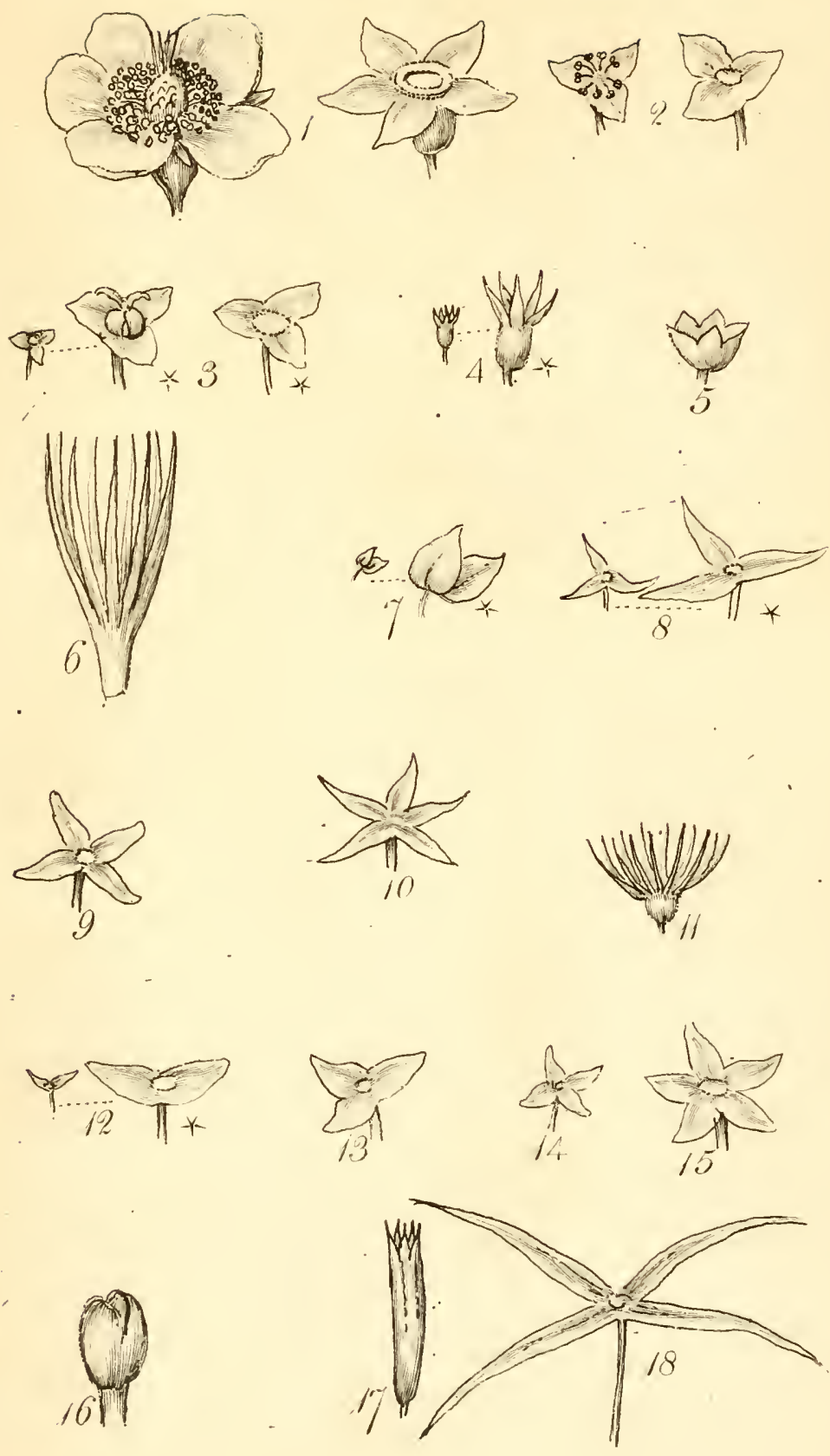



$$
\text { Balyx. Tat:y }
$$
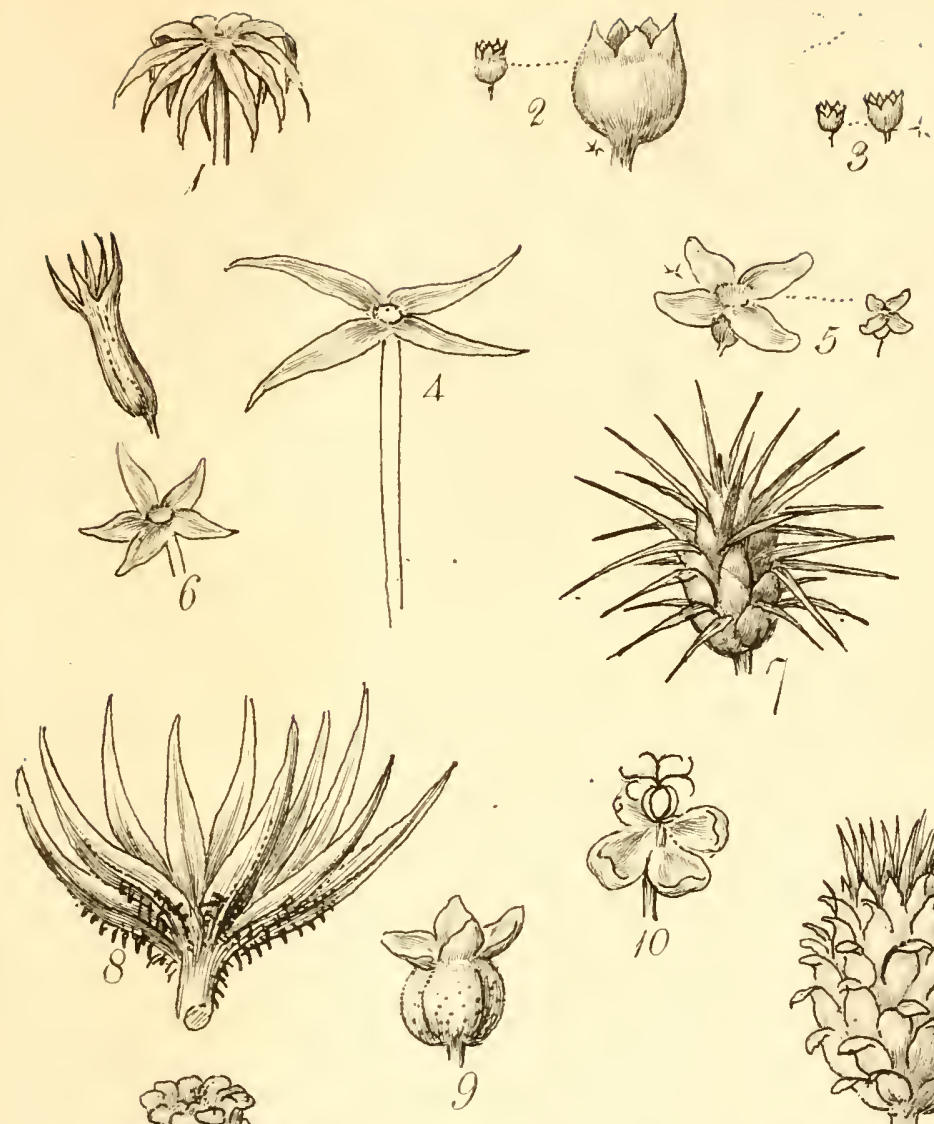

$$
\text { . }
$$





$$
\text { Gmetierum. }
$$
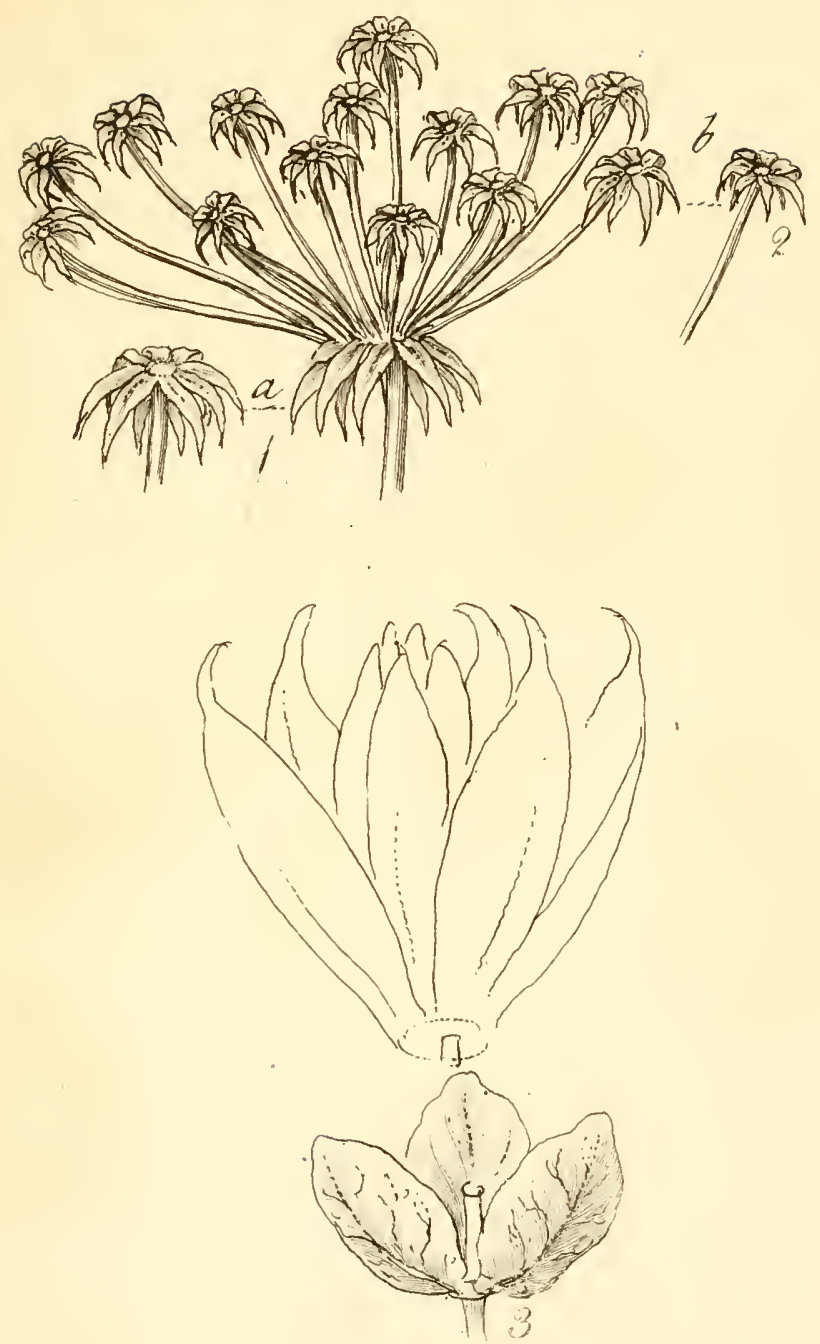



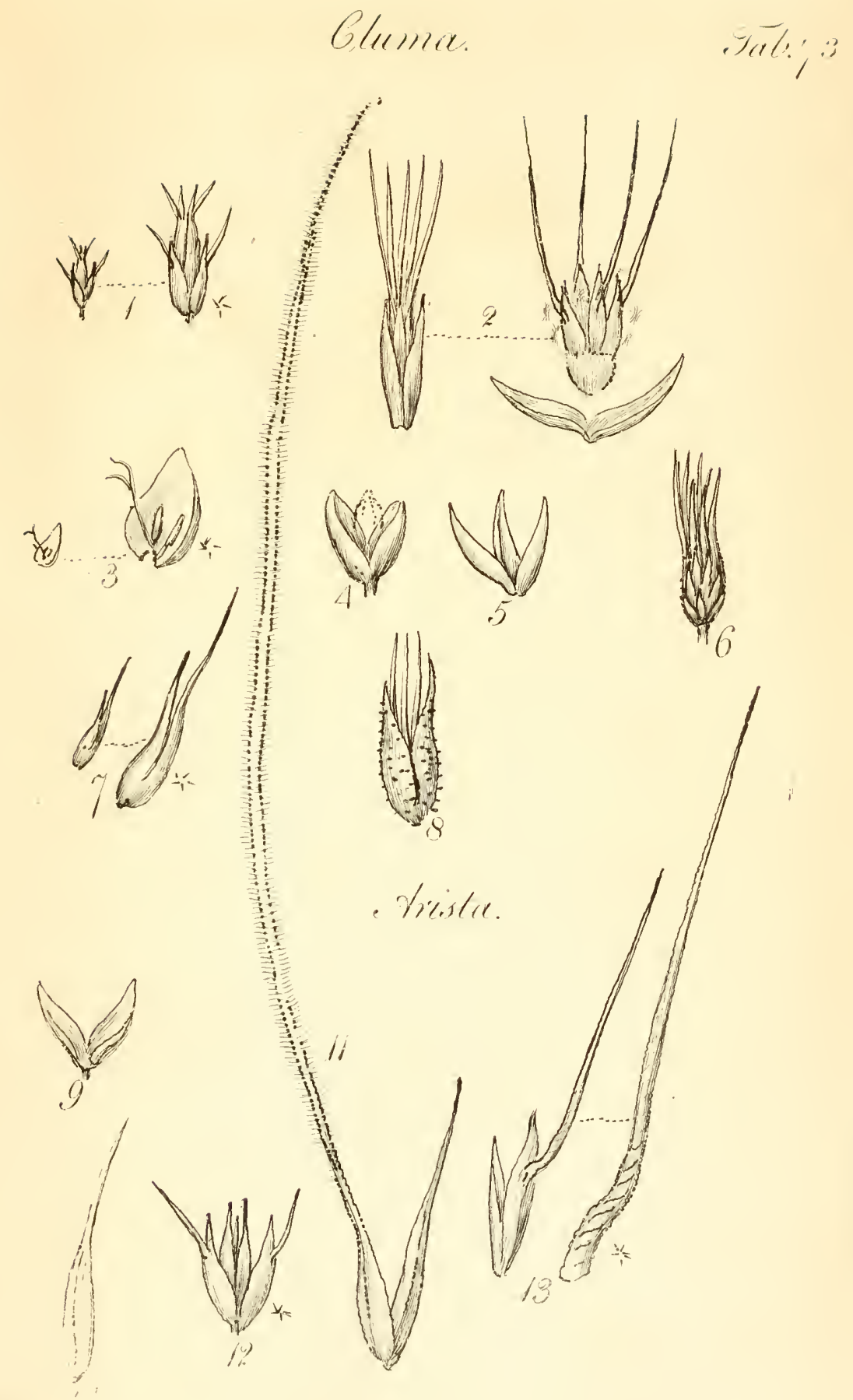



stmenturn \& sfurather.

Jat:y-1

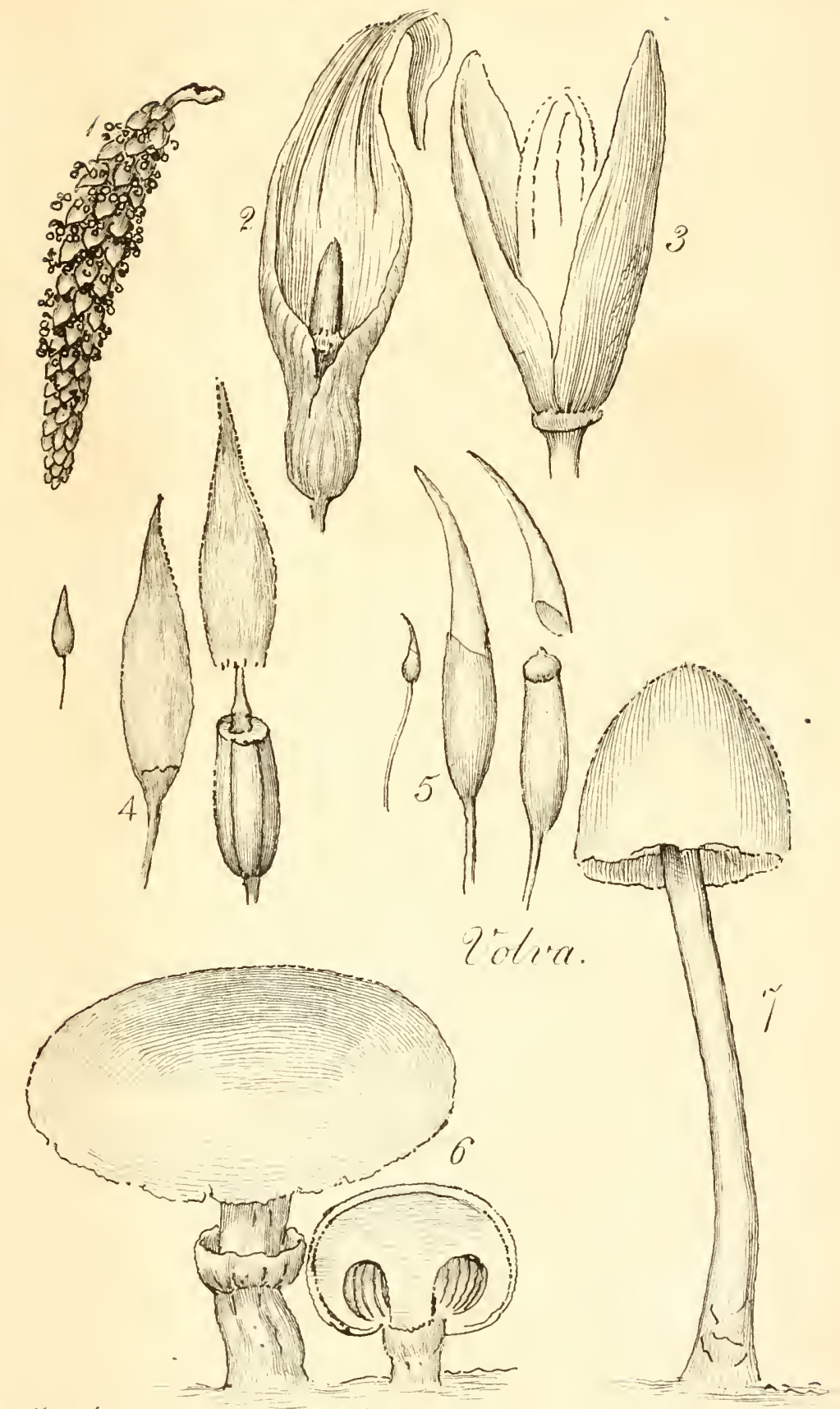



Corclle.
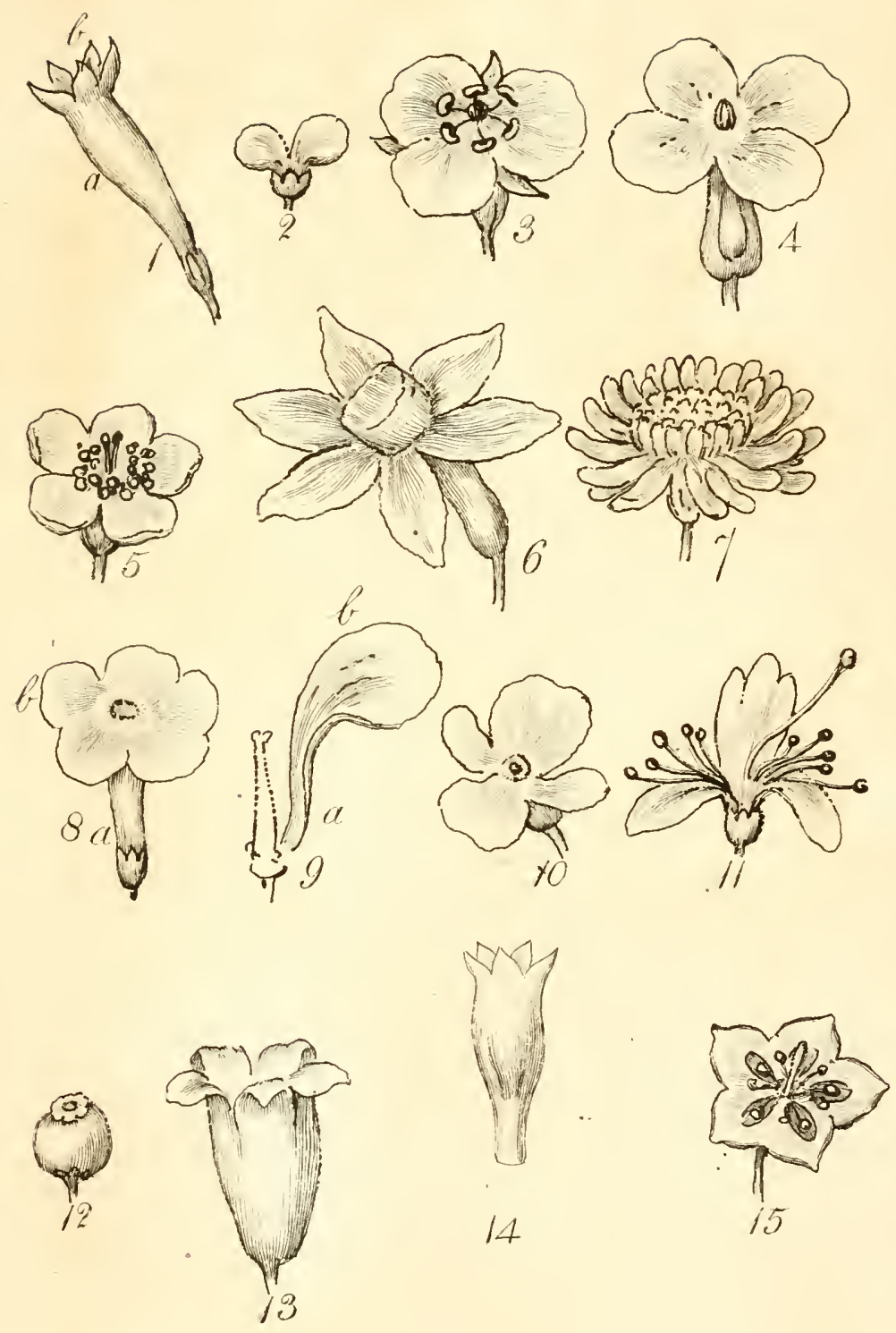

Gerolle.
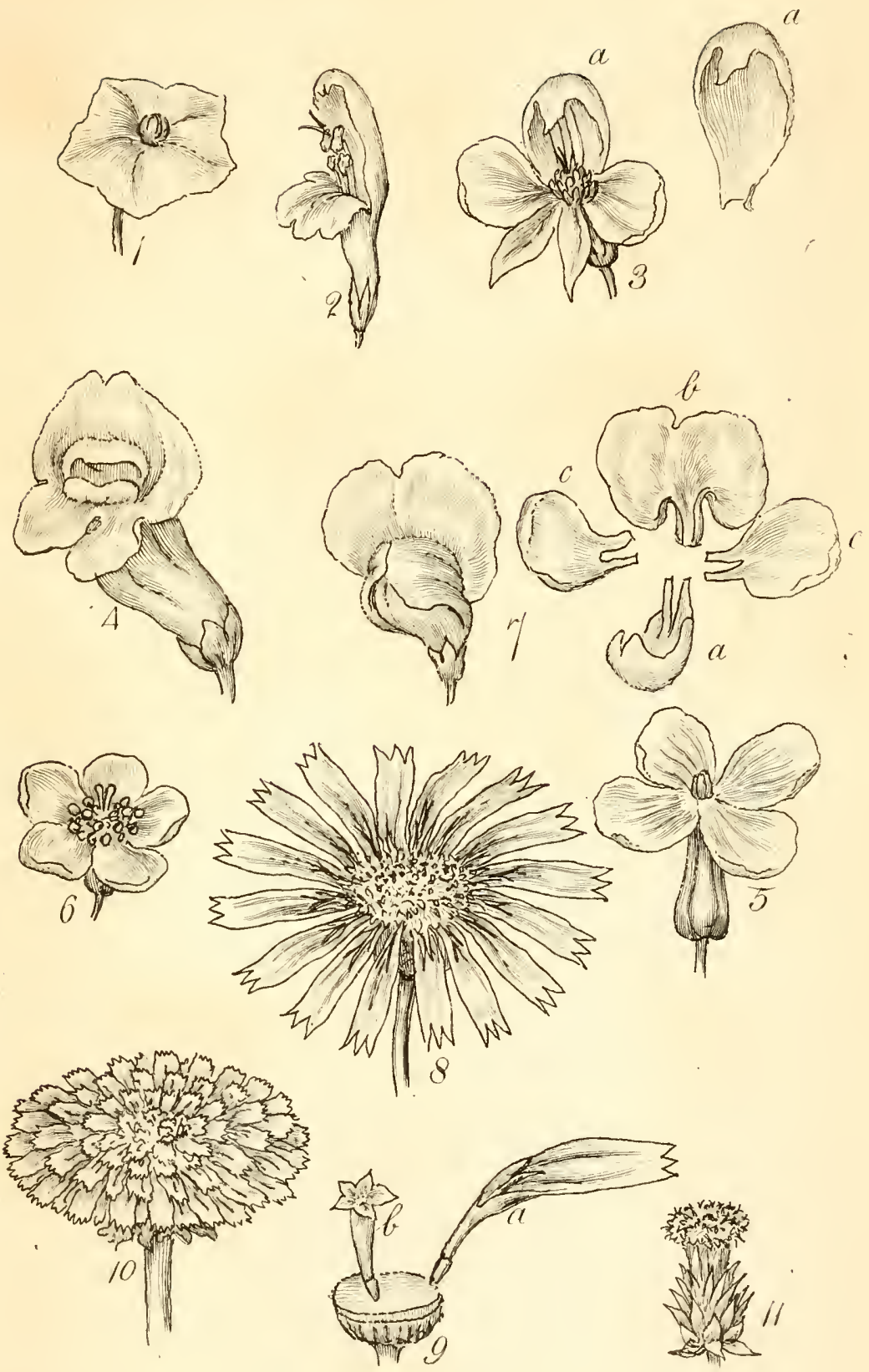



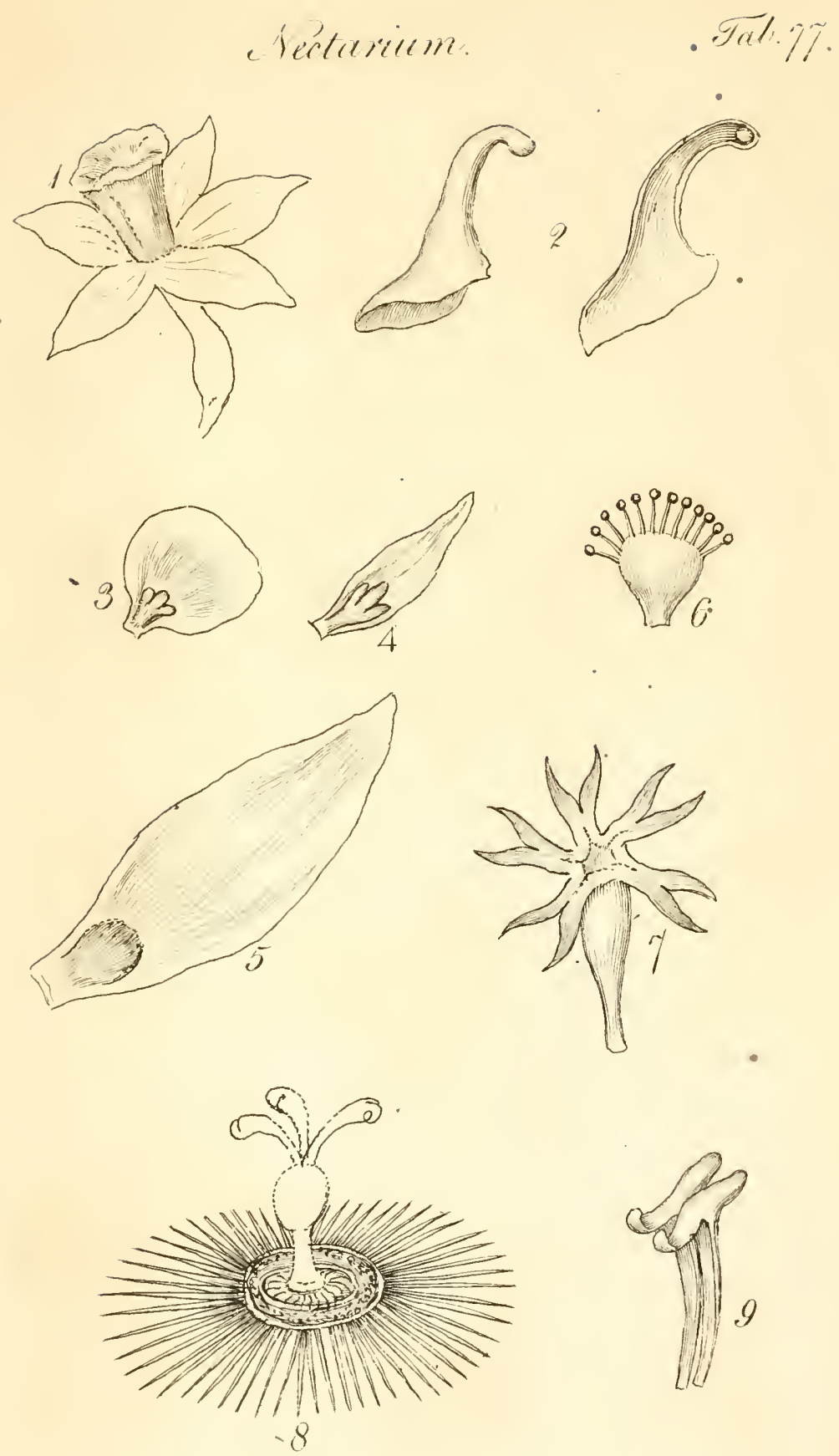

in $\left\|_{11}\right\|_{1} \cdot \mathrm{del}$. 

Stamina \&e Muthera.

अấ, नेc
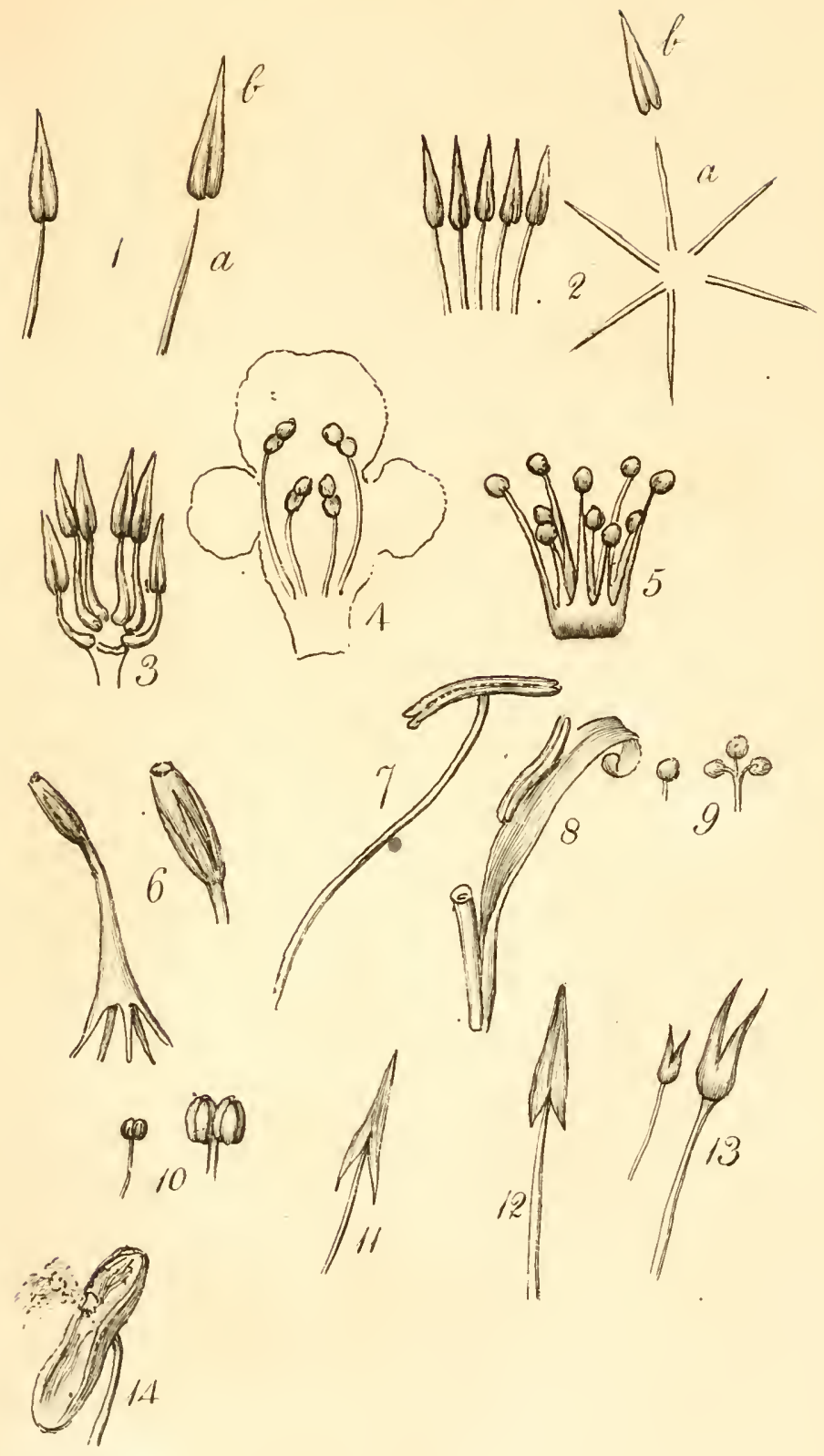

Fistillum:
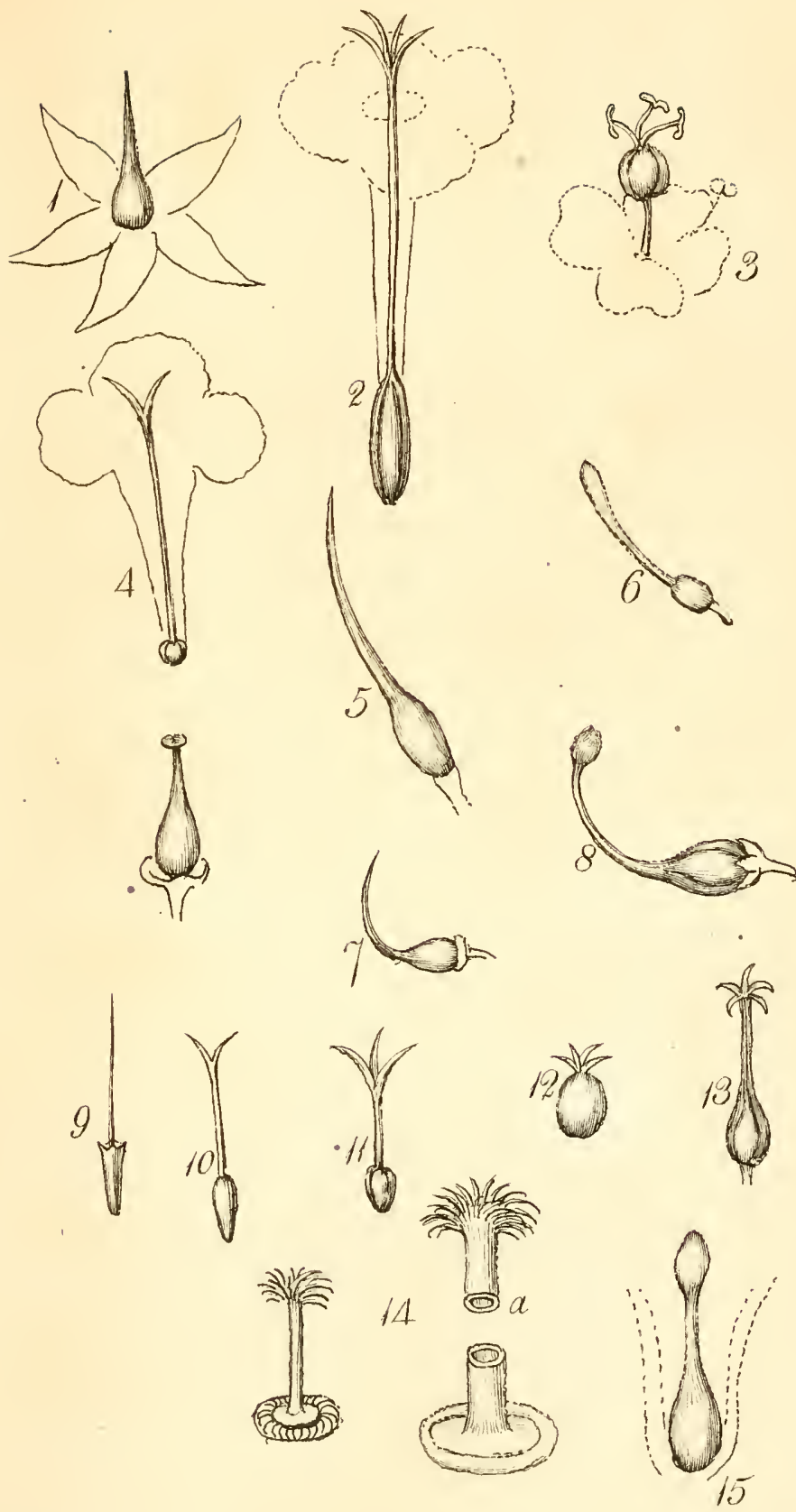

Tementellm

8,93009

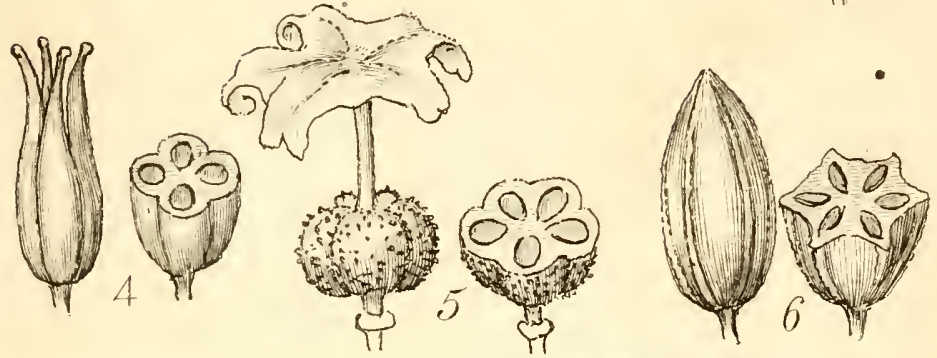

(1)
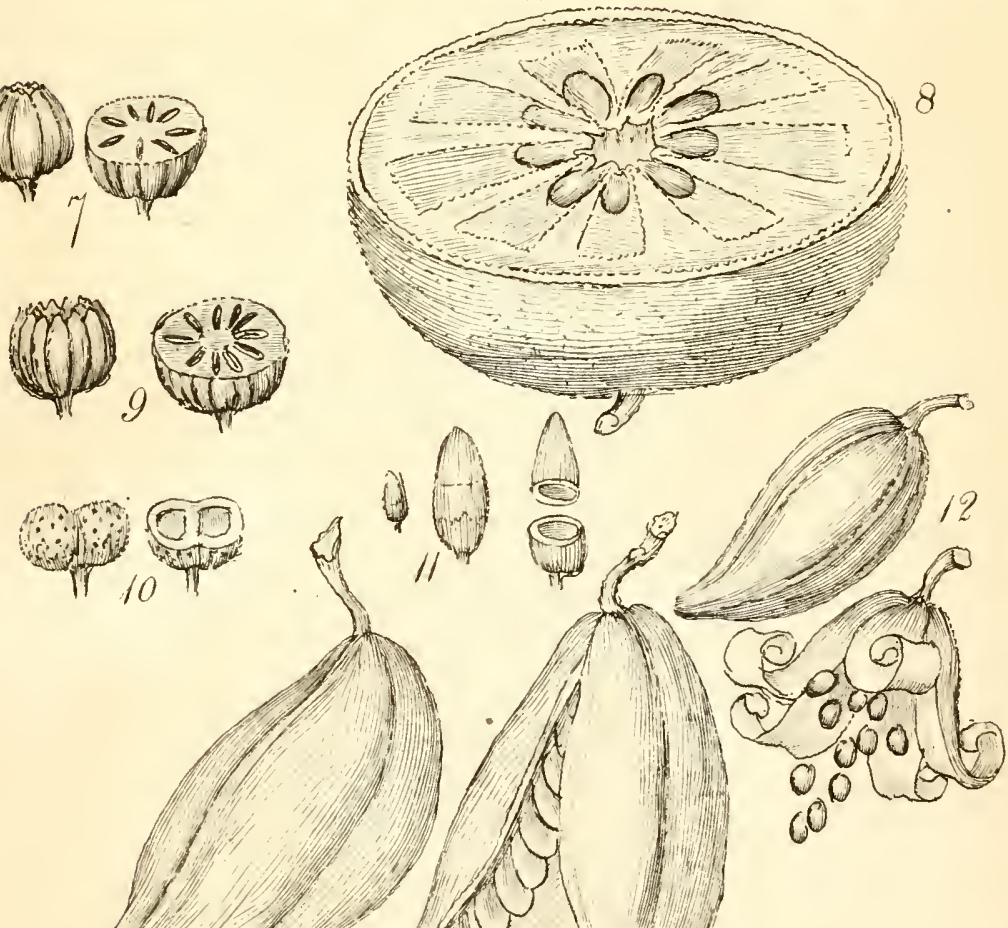

Pericarfium.

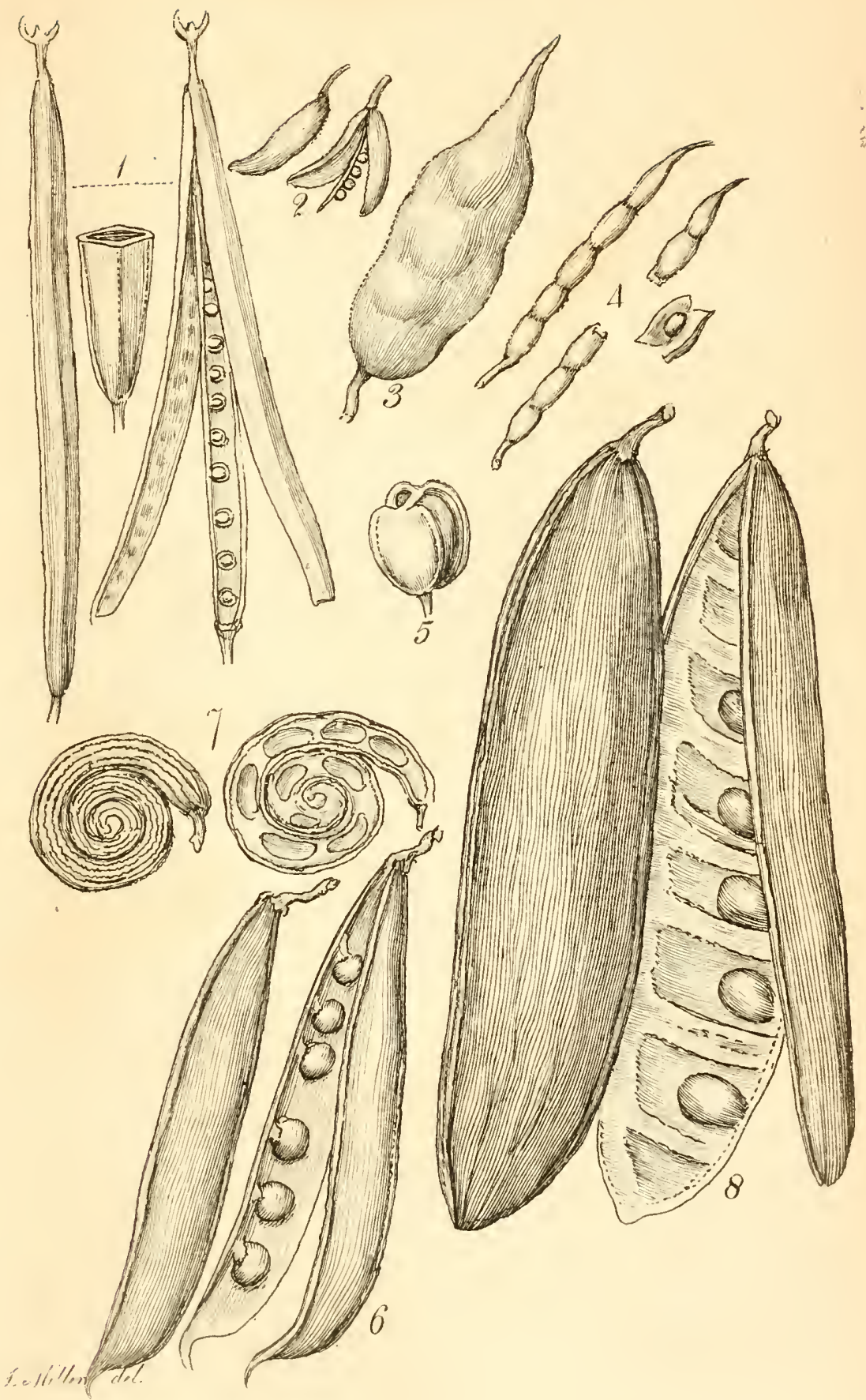




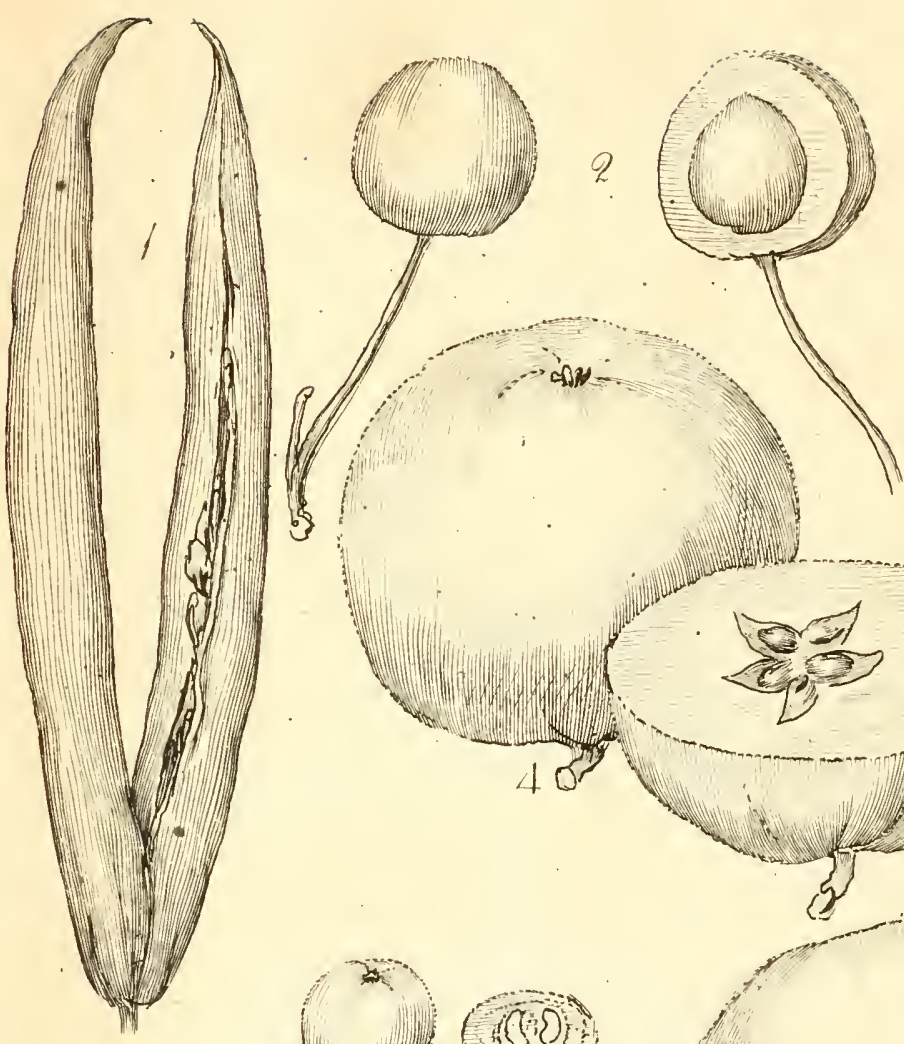

rfong
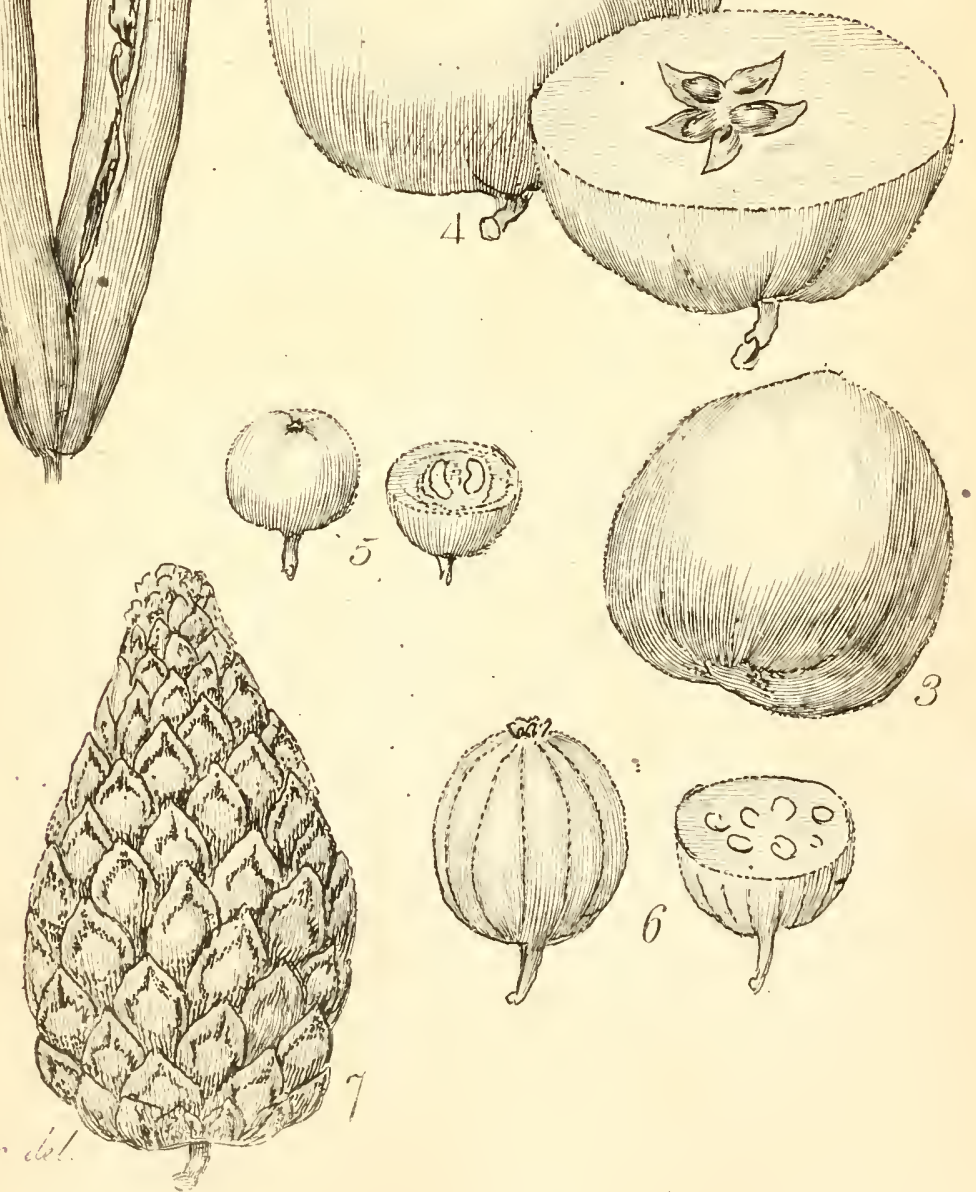



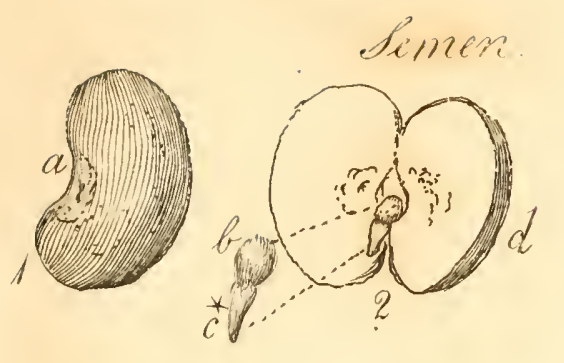

Fat. 8:3.
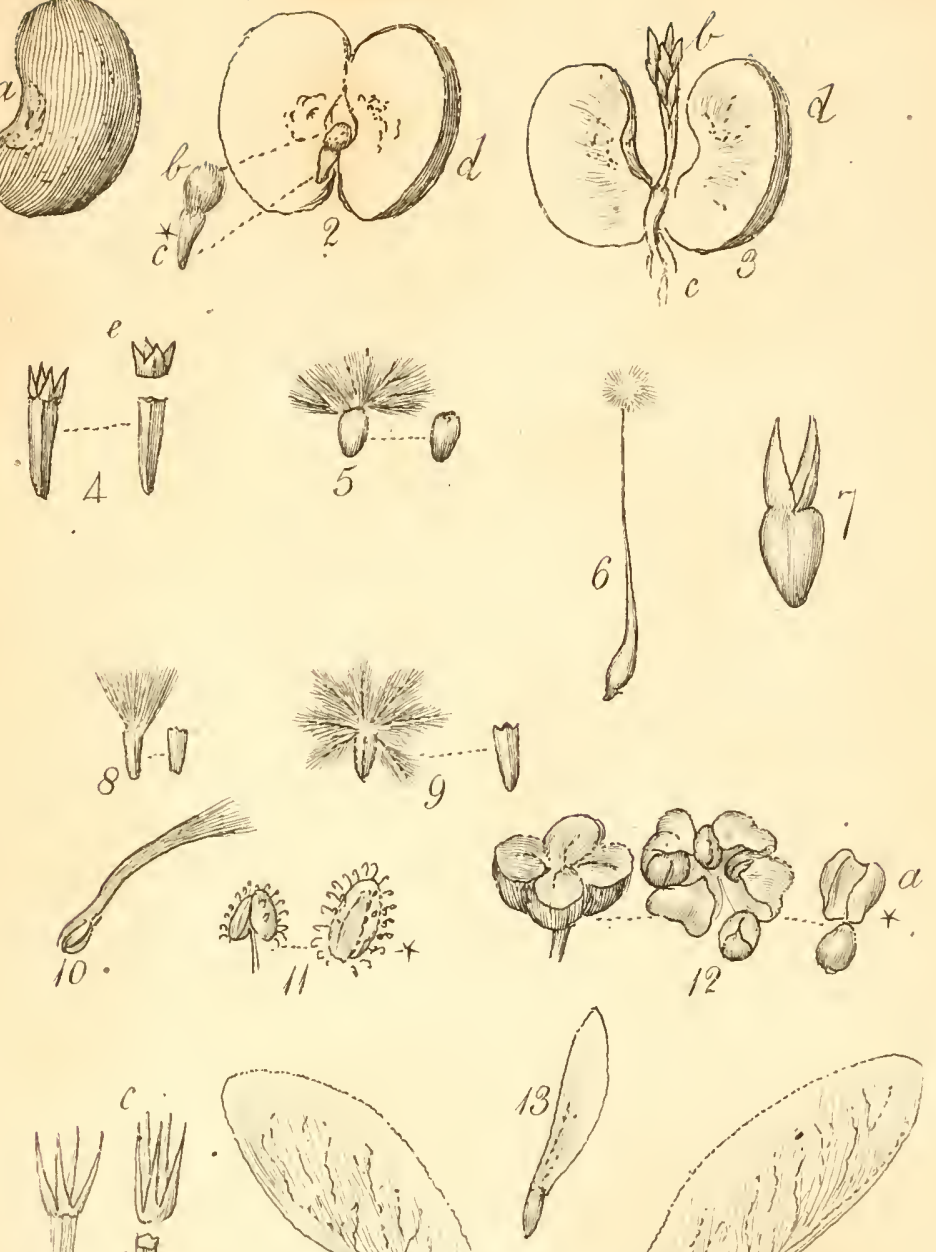

, 4 if
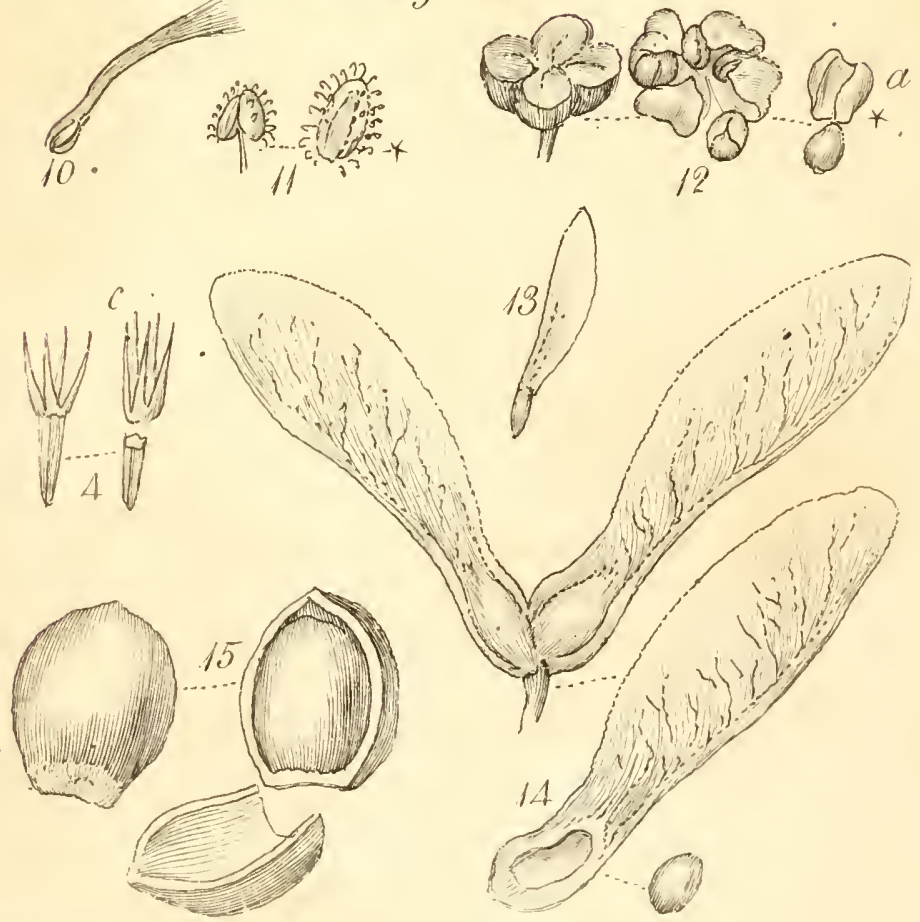

Receptrectelem
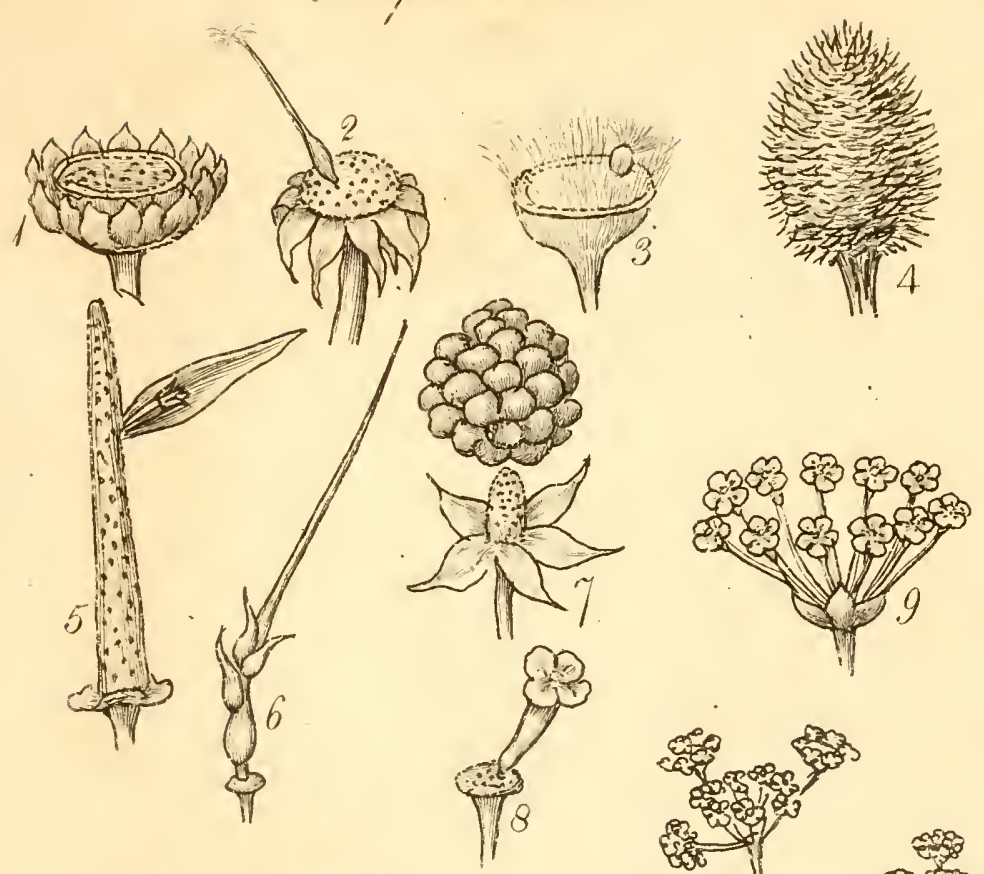

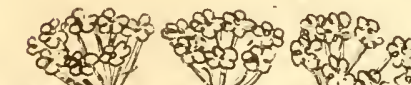
हैon

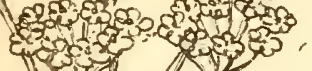

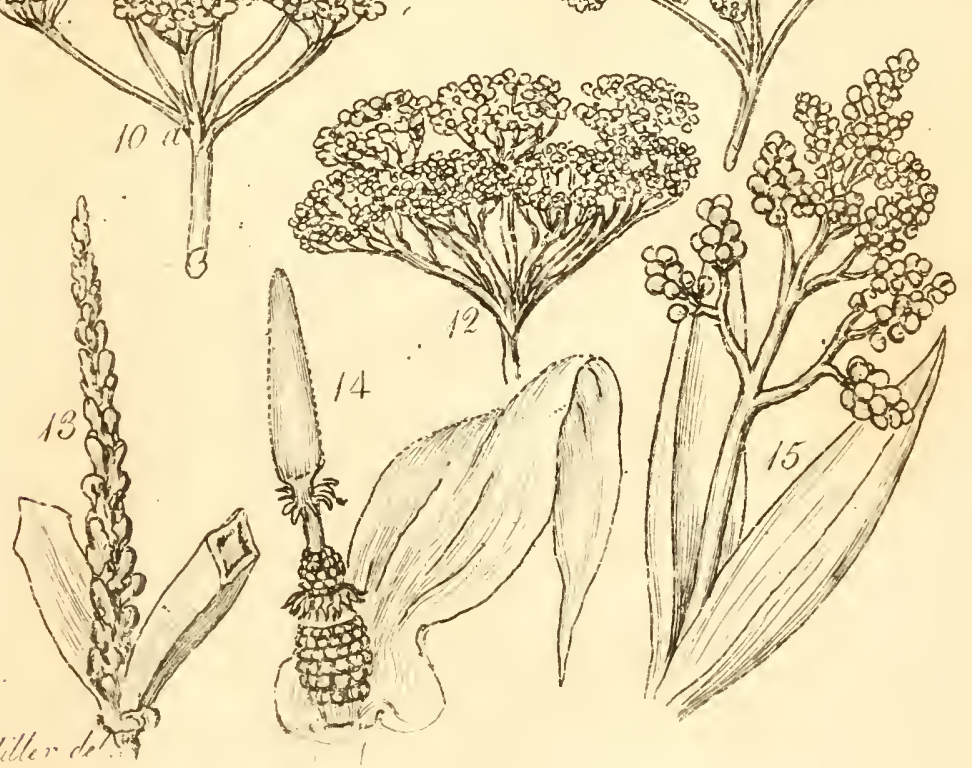



负ullus

Sat: 5.5

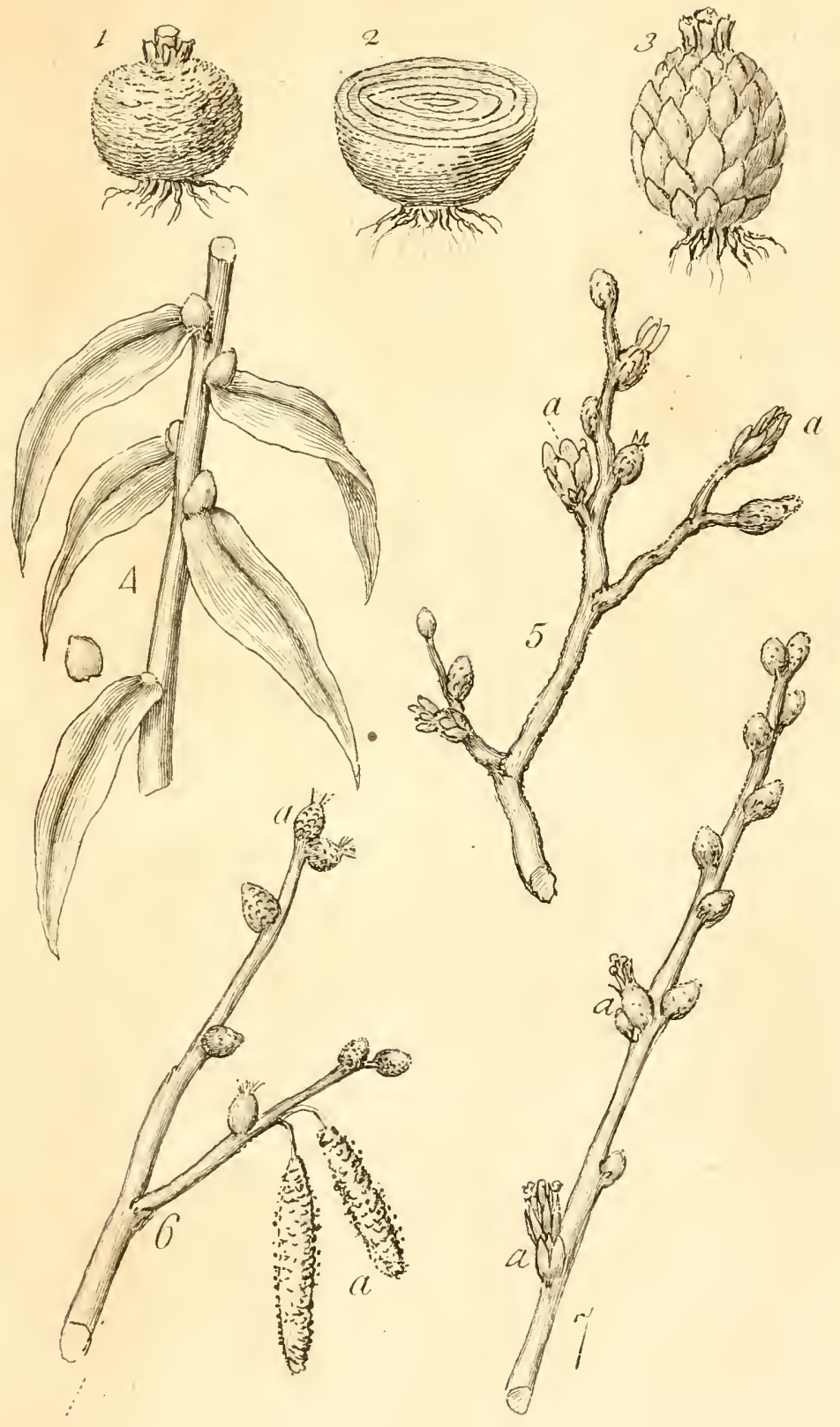



Oemato

rat: 80
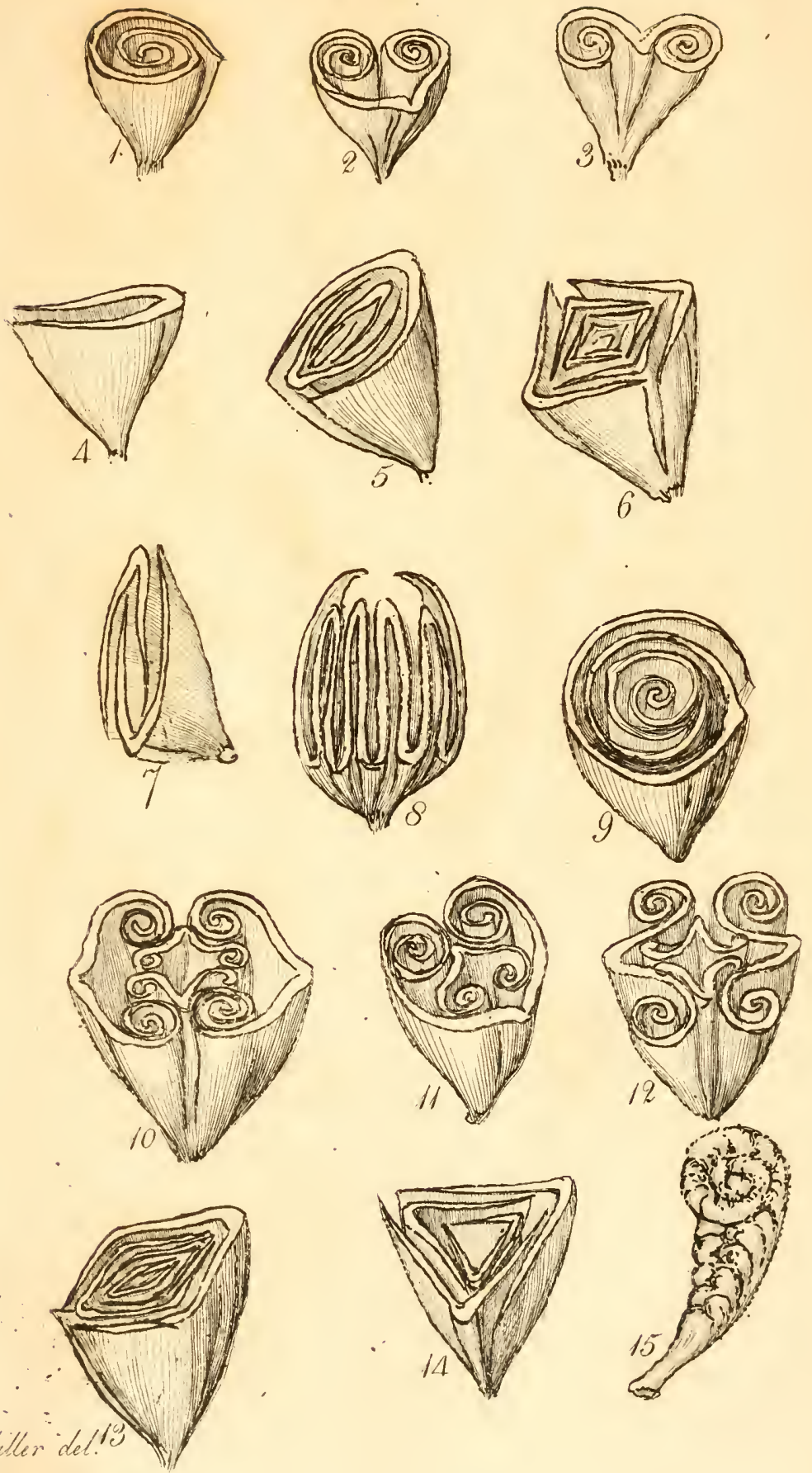


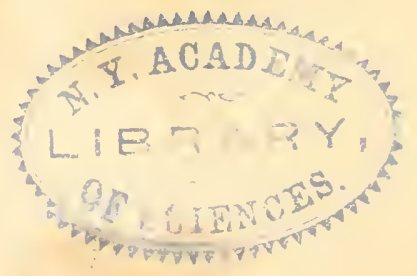






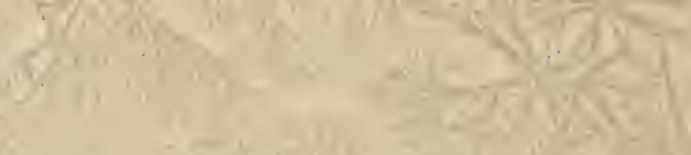

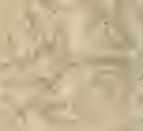

2

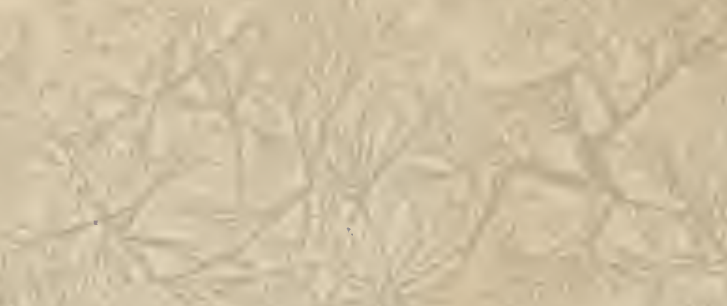

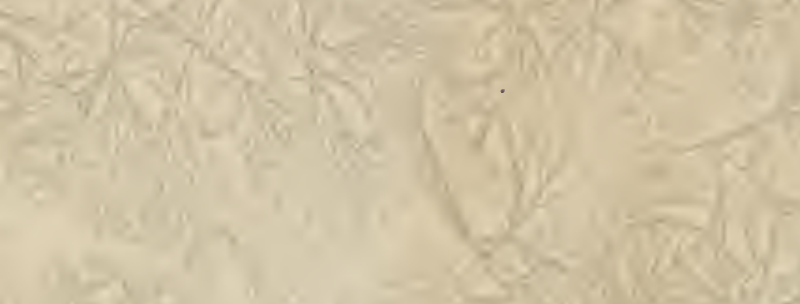

(2)

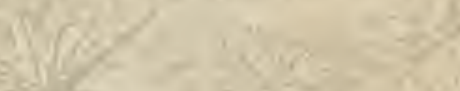

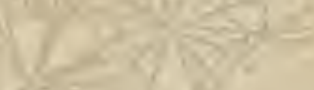

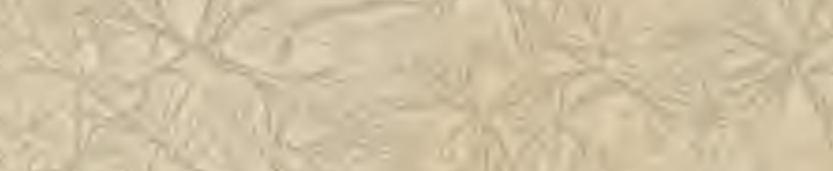

13 
Muller, Johann Seba/An illustration of $t$

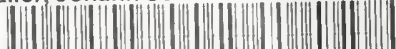

35185000913069 
